\title{
Nuclear Activity in a Volume-Limited Sample of Nearby Galaxies
}

\author{
Paulina Lira
}

Doctor of Philosophy

The University of Edinburgh 1999 
This thesis is my own composition except where indicated in the text.

July 5, 1999 


\section{Acknowledgements}

I want to say thank-you to my boss, Andy Lawrence, for his invaluable insight into the world of Astronomy and scientific research, and to Rachel Johnson, for her endless emails full of vital information. Without their help I would be living under a bridge by now.

Thanks to the people at the Institute for Astronomy for their friendship, especially to Peter, Liz, Alan, Eelco, Andy, and the gentlemen at the lodge; and of course to my PhDmates...Alison, Elese, Jo, Elspeth, Sujata, Louisa, Helen, Licia, Mike, Bill, Matt, Richard, Simon, Peter, Robert, Kenton....and some grown ups like Omar and Isobel...just to name a few.

Thanks to my family and friends back in my homeland for all the love and support they have given me from the long distance of $11776 \mathrm{~km}$.

Finally, thanks to Andrew, for having dinner ready every time I came home after hours of hard work writing this thesis, for reading every single paragraph of it to correct my English, for encouraging me to keep going so many times, but more importantly, for his unconditional love.

Oh! and I should not forget PPARC and Fundación Andes which paid for my University fees and fed me for 3 years of my life. 


\section{Abstract}

This thesis reports on the study of a volume-limited sample of 46 galaxies. It forms part of a continuing, multiwavelength program to assess the prevalence of low luminosity nuclear activity in galaxies with a wide range of sizes. X-ray observations are used, together with images at other wavelengths from previous work, to create a list of possible sites of nuclear activity. Optical spectra of these targets are analysed. The X-ray and optical properties of the nuclear regions, and their relationship with the host galaxies, are investigated. Finally, one particular object, NGC 4395, is described in detail.

ROSAT X-ray images were studied for 29 galaxies. Contour maps are presented for all galaxies with detected emission and fluxes are given for all observed point sources. Upper limits are given for galaxies with no detected nuclear sources. In general, X-ray nuclear sources are found to be extremely common. Their fluxes correlate strongly with the host galaxy luminosity (the analysis includes results from the literature for another 4 galaxies).

Long slit, high resolution, $\sim 3600-6800 \AA$ optical spectra were obtained for all the nuclear targets identified during careful examination of radio, IR, optical and X-ray images. Spectra are presented for each target and emission line fluxes are measured. Emission line diagnostic diagrams are used to investigate the nature of the ionising sources of the line-emitting targets. Results are compared with the spectral classification given by ? for galaxies common to both surveys and objects without previous observations are classified. The fraction of optically identified AGN in the sample is obtained.

Based on the observed correlation between X-ray and host galaxy luminosity, the properties of the nuclear X-ray sources are investigated. Nuclear $\mathrm{H} \alpha$ and $\mathrm{X}$-ray luminosities are compared with relationships established for more luminous AGN and with predictions from starburst models. Correlations are also found between nuclear $\mathrm{H} \alpha$ luminosities and host absolute magnitudes for nuclei optically classified as starbursts or AGN. Tentative explanations for the observed correlations are explored.

As part of the multiwavelength project, optical spectra and X-ray images of NGC 4395, the least luminous known Seyfert1, were obtained. The ROSAT observations show a change in the X-ray flux by a factor of $\sim 2$ in 15 days. The spectra show a change in both the 
level and shape of the continuum, becoming bluer when brighter. A power law fit to the observations shows that the spectral shape changes between a low state (with $\alpha \sim 2$ ), a medium state $(\alpha \sim 1)$, and a high state $(\alpha \sim 0)$. A week of ground-based optical broad band monitoring of NGC 4395 has also given evidence of a variation in the flux by $\sim 20 \%$ in less than 24 hours. 


\section{Contents}

1 Introduction $\quad 13$

1.1 How common are AGN? . . . . . . . . . . . . . . . . 13

1.1 .1 Recognising AGN . . . . . . . . . . . . . . 14

1.2 Relationship with host galaxy properties . . . . . . . . . . . 16

1.2 .1 Correlation with host galaxy luminosity . . . . . . . . 16

1.2.2 Correlation with host galaxy morphology type . . . . . . . 17

1.3 Cosmological context . . . . . . . . . . . . . . 18

1.4 Dormant black holes . . . . . . . . . . . . . . . . . . . 19

1.5 A new project: the multiwavelength study of a volume-limited sample of

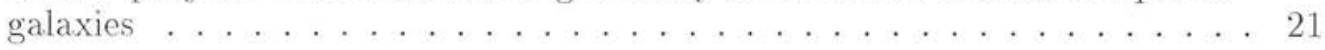

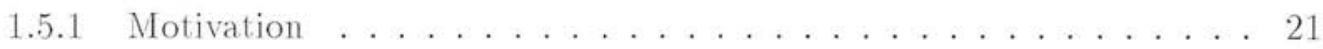

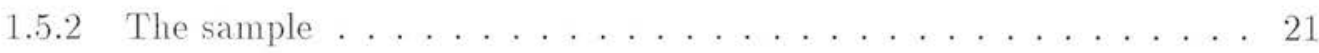

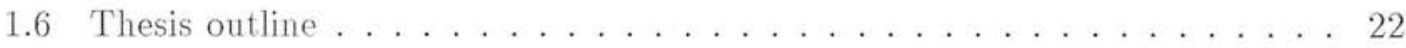

1.7 Sources of X-ray emission . . . . . . . . . . . . . . . 22

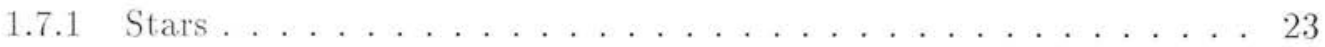

1.7 .2 Cataclysmic variables ...................... 23

1.7 .3 Supernova remnants . . . . . . . . . . . . . 23

1.7 .4 X-ray binaries. . . . . . . . . . . . . 25

1.8 X-rays from the nearest galaxies . . . . . . . . . . . . . 26

$1.8 .1 \mathrm{NGC} 224(\mathrm{M} 31) \ldots \ldots \ldots \ldots \ldots \ldots$ 
1.8 .2 The Magellanic Clouds . . . . . . . . . . . . . . . 27

2 High resolution X-ray imaging 29

2.1 The Roentgen Satellite . . . . . . . . . . . . . . . . . . . . . . . 29

2.1 .1 The High Resolution Imager . . . . . . . . . . . . . . 30

2.2 Data reduction and analysis . . . . . . . . . . . . . . 34

2.2 .1 Standard processing at MPE . . . . . . . . . . 36

2.2 .2 Observations . . . . . . . . . . . . . . 36

2.2 .3 Analysis procedure . . . . . . . . . . . . . . . 37

2.3 The X-ray Atlas . . . . . . . . . . . . . . . . . . 41

2.4 Notes on individual objects .................... 61

$\begin{array}{lll}3 & \text { Optical spectroscopic observations } & 77\end{array}$

3.1 Selection of targets and astrometry . . . . . . . . . . . 77

3.1 .1 Optical sources in JKT images . . . . . . . . . . . . . 77

3.1 .2 Optical Sources without JKT Images . . . . . . . . . . . . . . 78

3.1.3 Radio sources without optical counterparts . . . . . . . . . . . 79

3.1.4 X-Ray sources without optical or radio counterparts . . . . . . . 79

3.2 The WHT and ISIS . . . . . . . . . . . . . . . . . 79

3.3 Observations, data reduction and calibration ............. 80

3.3 .1 Observing runs . . . . . . . . . . . . . . . 80

3.3 .2 Data reduction .......................... 82

3.3.3 Spectrum extraction and calibration .............. 85

3.4 The spectral atlas . . . . . . . . . . . . . . . . 90

3.5 Notes on individual objects . . . . . . . . . . . . . . . . . 119

4 NGC 4395, the feeblest known Seyfert 1 nucleus 131

4.1 Introduction . . . . . . . . . . . . . . . . . . 131 
4.2 .1 HRI data . . . . . . . . . . . . . . . . . . . . . . 132

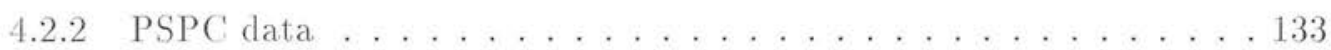

4.2 .3 Optical spectroscopy . . . . . . . . . . . . . . . . 134

4.2.4 Ground-based broad-band observations . . . . . . . . . . 135

4.2 .5 HST WFPC2 observations . . . . . . . . . . . 136

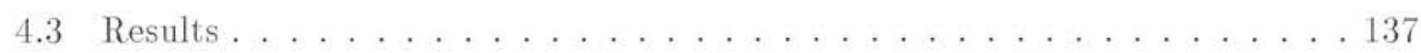

4.3.1 X-ray imaging and spectral analysis . . . . . . . . . . 137

4.3.2 Analysis of optical spectra . . . . . . . . . . . . . . 139

4.3 .3 Broad-band variability . . . . . . . . . . . . 145

4.3 .4 Analysis of HST images . . . . . . . . . . . . . . . 147

4.4 Discussion . . . . . . . . . . . . . . . . . . . . . . 149

4.4 .1 Spectral energy distribution . . . . . . . . . . . . . . . 149

4.4 .2 Optical spectroscopic variability . . . . . . . . . . 152

4.4 .3 Fast variability . . . . . . . . . . . . . . 154

4.4 .4 The ionizing continuum . . . . . . . . . . . 155

4.4.5 Black hole mass in NGC4395 . . . . . . . . . . . 156

4.4 .6 X-ray loudness . . . . . . . . . . . . . . . . . 158

4.4.7 Comparison with other low luminosity AGN . . . . . . . . . . 159

4.4.8 An advective-dominated accretion flow in NGC 4395 . . . . . . 161

4.4.9 Constraints on nuclear starburst models . . . . . . . . . . . . 161

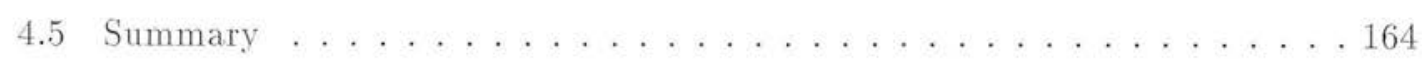

$5 \quad$ Nuclear emission from a complete sample of nearby galaxies $\quad 167$

5.1 Optical classification of emission line spectra . . . . . . . . . 167

5.1 .1 Diagnostic diagrams . . . . . . . . . . . . . . . 171

5.1 .2 The final spectral classification . . . . . . . . . . . . 178 
5.2 The AGN population . . . . . . . . . . . . . . . . 178

5.2 .1 Comparison with the normal galaxy population . . . . . . . . . 180

5.2 .2 The luminosity dependent fraction of AGN . . . . . . . . . . 182

5.3 Nuclear X-ray sources . . . . . . . . . . . . . . . . . . . . . 183

5.3 .1 X-ray nuclear and host galaxy luminosities . . . . . . . . . . 184

5.3 .2 Super-luminous X-ray off-nuclear sources . . . . . . . . . . . 187

5.4 The nature of the nuclear sources . . . . . . . . . . . . . . . . 189

5.4 .1 The AGN hypothesis . . . . . . . . . . . . . . 190

5.4 .2 The star formation model . . . . . . . . . . . . . . . 196

5.4 .3 Conclusions . . . . . . . . . . . . . . . . . . 211

6 Summary

A X-ray emitting mechanisms.

A.1 Black Body Radiation . . . . . . . . . . . . . . . . . 215

A.2 Thermal Free-Free or Bremsstrahlung Emission . . . . . . . . . . . . . 216

A.3 Synchrotron radiation . . . . . . . . . . . . . . . . 216

A.4 Inverse Compton Scattering . . . . . . . . . . . . . . 217

B Statistical formulism

B.1 Confidence limits for Poisson Statistics . . . . . . . . . . . . . . . . 219

B.2 The Kolmogorov-Smirnov test . . . . . . . . . . . . . . . . . . 220 


\section{List of Figures}

2.1 ROSAT HRI effective area . . . . . . . . . . . . . . 30

2.2 Parametric form of the HRI PSF . . . . . . . . . . . . 31

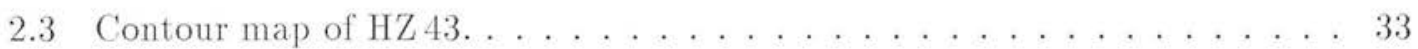

2.4 Comparison of two observed PSFs . . . . . . . . . . . . 34

2.5 NGC 147 X-ray contours overlaid on optical JKT image . . . . . . . . . 46

2.6 NGC 185 X-ray contours overlaid on optical JKT image . . . . . . . . . 46

2.7 NGC205 X-ray contours overlaid on optical JKT image . . . . . . . . . . 47

2.8 NGC221 X-ray contours overlaid on optical JKT image . . . . . . . . 47

2.9 NGC247 X-ray contours overlaid on optical JKT image . . . . . . . . . 48

2.10 NGC 404 X-ray contours overlaid on optical JKT image . . . . . . . . . . 48

2.11 IC 342 X-ray contours overlaid on optical JKT image . . . . . . . . . . . 49

2.12 NGC 1560 X-ray contours overlaid on optical DSS image . . . . . . . . . 49

2.13 NGC 2366 X-ray contours overlaid on optical JKT image . . . . . . . . . 50

2.14 NGC 2403 X-ray contours overlaid on optical JKT image . . . . . . . . . . 50

2.15 NGC 2976 X-ray contours overlaid on optical JKT image . . . . . . . . . . 51

2.16 A $0951+68$ X-ray contours overlaid on optical JKT image . . . . . . . . 51

2.17 UGC 6456 X-ray contours overlaid on optical JKT image . . . . . . . . . . 52

2.18 NGC 3738 X-ray contours overlaid on optical JKT image . . . . . . . . 52

2.19 NGC 4136 X-ray contours overlaid on optical JKT image . . . . . . . . . . 53

2.20 NGC 4144 X-ray contours overlaid on optical JKT image . . . . . . . . . 53 
2.21 NGC 4150 X-ray contours overlaid on optical JKT image . . . . . . . . 54

2.22 NGC 4236 X-ray contours overlaid on optical JKT image . . . . . . . . . . 54

2.23 NGC 4244 X-ray contours overlaid on optical JKT image . . . . . . . 55

2.24 NGC 4395 X-ray contours overlaid on optical DSS image . . . . . . . . . 55

2.25 NGC 4605 X-ray contours overlaid on optical JKT image . . . . . . . . . 56

2.26 NGC 4736 X-ray contours overlaid on optical JKT image . . . . . . . . 56

2.27 NGC 4826 X-ray contours overlaid on optical JKT image . . . . . . . . 57

2.28 NGC 5204 X-ray contours overlaid on optical JKT image . . . . . . . 57

2.29 NGC 5236 X-ray contours overlaid on optical JKT image . . . . . . . 58

2.30 NGC 5238 X-ray contours overlaid on optical JKT image . . . . . . . 58

2.31 NGC 5457 X-ray contours overlaid on optical JKT image . . . . . . . . 59

2.32 NGC 6503 X-ray contours overlaid on optical JKT image. . . . . . . . . 59

2.33 NGC 6946 X-ray contours overlaid on optical JKT image . . . . . . . . 60

2.34 IC 342 observed X-ray profile . . . . . . . . . . . . . . 64

2.35 I-band image of $\mathrm{UGC} 6456 \ldots \ldots \ldots \ldots \ldots \ldots$

2.36 Apertures used in NGC4150 . . . . . . . . . . . . . . 67

2.37 NGC 4736 observed X-ray profile . . . . . . . . . . . . . 68

2.38 NGC 4826 observed X-ray profile . . . . . . . . . . . . 69

2.39 NGC 5236 observed X-ray profile . . . . . . . . . . . . . . 71

2.40 NGC 5457 observed X-ray profile . . . . . . . . . . . 72

2.41 NGC 6503 X-ray countours . . . . . . . . . . . . . 73

2.42 NGC 6503 observed X-ray profile . . . . . . . . . . . . 74

2.43 NGC 6946 observed X-ray profile . . . . . . . . . . . . . 75

3.1 Loral cosmetic defect . . . . . . . . . . . . . . . . . . . . . . 81

3.2 Overscan region obtained with the LORAL CCD . . . . . . . . 83

3.3 Profiles of bias frames . . . . . . . . . . . . . . . 84 
3.4 Dispersion in $C_{\lambda}$ from the standard star observations . . . . . . . . . 88

3.5 Nuclear spectroscopy targets. . . . . . . . . . . . . . . . . . . 95

3.6 Nuclear spectroscopy targets. . . . . . . . . . . . . . . . 96 96

3.7 Nuclear spectroscopy targets. . . . . . . . . . . . . . . . . . . . 97

3.8 Spectral atlas . . . . . . . . . . . . . . . . . . . . . . . . 98

3.9 Spectral atlas . . . . . . . . . . . . . . . . . . . . . 99

3.10 Spectral atlas . . . . . . . . . . . . . . . . . . . . . . . . . . .

3.11 Spectral atlas . . . . . . . . . . . . . . . . . 101

3.12 Spectral atlas . . . . . . . . . . . . . . . . . . 102

3.13 Spectral atlas . . . . . . . . . . . . . . . . . . . . . . . . . . . .

3.14 Spectral atlas . . . . . . . . . . . . . . . . . 104

3.15 Spectral atlas . . . . . . . . . . . . . . . . 105

3.16 Spectral atlas . . . . . . . . . . . . . . . . . . . . . . . . . . . .

3.17 Spectral atlas . . . . . . . . . . . . . . . . . . . . . . . .

3.18 Spectral atlas . . . . . . . . . . . . . . . . . . . 108

3.19 Spectral atlas . . . . . . . . . . . . . . . . . . 109

3.20 Spectral atlas . . . . . . . . . . . . . . . . . . . . . . . . . . . .

3.21 Spectral atlas . . . . . . . . . . . . . . . . . . . . . . . . .

3.22 Spectral atlas . . . . . . . . . . . . . . . . . . . . . . . . . .

3.23 Spectral atlas . . . . . . . . . . . . . . . . . . . . . . . . . . . .

3.24 Spectral atlas . . . . . . . . . . . . . . . . . . . 114

3.25 Spectral atlas . . . . . . . . . . . . . . . . . . . . . . . . . . . .

3.26 Spectral atlas . . . . . . . . . . . . . . . . . 116

3.27 Spectral atlas . . . . . . . . . . . . . . . . . . 117

3.28 Spectral atlas . . . . . . . . . . . . . . . . . . . . . . . . . . .

3.29 Detail of the Maffei I spectrum . . . . . . . . . . . . . . . 122 
3.30 Detail of the NGC2366 V4 spectrum . . . . . . . . . . . 123

3.31 Detail of the UGC $6456 \mathrm{~V} 4$ spectrum . . . . . . . . . . . . . 125

4.1 NGC 4395 optical spectra . . . . . . . . . . . . . . . . 138

4.2 Gaussian profile fitting to $H \alpha$ and $H \beta \ldots \ldots \ldots \ldots$. . . . . . . . . . . . .

4.3 Narrow line flux ratios . . . . . . . . . . . . . . . . . . . . . . . 142

4.4 Fitted continuum levels . . . . . . . . . . . . . . . . . . . . . . 144

4.5 JKT $I$ and $B$ relative photometry . . . . . . . . . . 146

4.6 HST PC 2 radial profiles . . . . . . . . . . . . . . . . . . 148

4.7 NGC 4395 spectral energy distribution . . . . . . . . . . . . 150

$4.8 \mathrm{H} \alpha+$ N II line profiles . . . . . . . . . . . . . . . . . . . 153

5.1 OII $\lambda 3727 /$ OIII $\lambda 5007$ vs NII $\lambda 6584 / \mathrm{H} \alpha \lambda 6503$ diagnostic diagrams . . . . . 173

5.2 OI $\lambda 6300 /$ OIII $\lambda 5007$ vs OII $\lambda 3727 /$ OII $\lambda 5007$ diagnostic diagram $\ldots \ldots . .176$

5.3 Luminosity distribution in the volume-limited sample . . . . . . . . . . 180

5.4 Luminosity distribution in the Palomar sample . . . . . . . . . . . . . 181

5.5 Fraction of AGN to total number of galaxies . . . . . . . . . . . . 182

5.6 Nuclear X-ray luminosity as a function of the host absolute magnitude . . . 185

5.7 Predicted X-ray cumulative luminosity functions . . . . . . . . . . . 186

5.8 Off-nuclear X-ray luminosities as a function of host galaxy luminosity . . . 187

5.9 X-ray versus $H \alpha$ nuclear luminosities . . . . . . . . . . . . . . . . . . 190

5.10 Nuclear radio power as a function of host galaxy absolute magnitude . . . . 193

5.11 Nuclear narrow $\mathrm{H} \alpha$ versus host luminosities for Seyfert galaxies . . . . . . . 193

5.12 Nuclear broad $\mathrm{H} \alpha$ versus host luminosities for Seyfert galaxies . . . . . . . . 194

5.13 Nuclear $\mathrm{H} \alpha$ versus host luminosities for HII region nuclei . . . . . . . . . 194

5.14 Star formation rate . . . . . . . . . . . . . . . . . . 197

5.15 Predicted and observed SN rate . . . . . . . . . . . . . . 199

5.16 Long slit spectroscopic images . . . . . . . . . . . . . . . . . 205 
5.17 Observed $\mathrm{H} \alpha$ and $\mathrm{X}$-ray extended profiles . . . . . . . . . . 207

5.18 Observed $\mathrm{H} \alpha$ and $\mathrm{X}$-ray extended profiles . . . . . . . . . . 208

5.19 Observed $\mathrm{H} \alpha$ and $\mathrm{X}$-ray extended profiles . . . . . . . . . . 209

5.20 X-ray versus $\mathrm{H} \alpha$ corrected luminosities . . . . . . . . . . . . . . . 210 


\section{List of Tables}

1.1 The volume-limited sample of galaxies . . . . . . . . . . . 28

2.1 Dependency of the PSF with energy . . . . . . . . . . . 32

2.2 ROSAT HRI observations . . . . . . . . . . . . . . . 35

2.3 Conversion factors for HRI flux calibrations . . . . . . . . . . 40

2.4 ROSAT HRI fluxes and luminosities . . . . . . . . . . . . . . 42

2.5 ROSAT HRI nuclear upper limits . . . . . . . . . . . . . . 44

2.6 HRI sources from the literature . . . . . . . . . . . . . 45

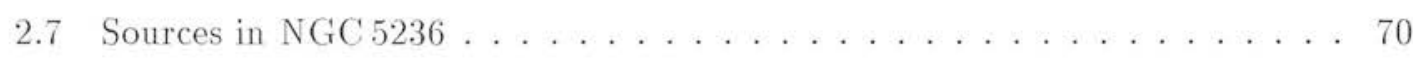

3.1 Journal of spectroscopic observations . . . . . . . . . . . 78

3.2 Set-up for the spectroscopy . . . . . . . . . . . . 80

3.3 Extinction during the run in July $1996 \ldots \ldots \ldots \ldots \ldots$. . . . . . . 82

3.4 Journal of calibration frames . . . . . . . . . . . . . 86

3.5 Calibration check: relative fluxes ... . . . . . . . . . . 92

3.6 Line fluxes . . . . . . . . . . . . . . . . . . . . . . . . . . 93

4.1 Journal of observations of $\mathrm{NGC} 4395 \ldots \ldots \ldots \ldots$

4.2 Narrow line fluxes . . . . . . . . . . . . . . . . . . . 134

4.3 Broad and narrow deblended line fluxes . . . . . . . . . . . . . 134

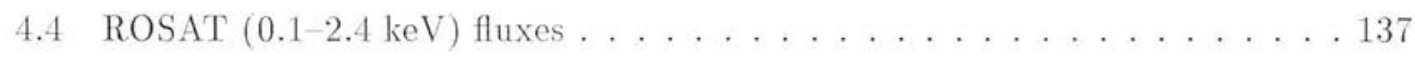

5.1 Galactic extinction . . . . . . . . . . . . . . 169 
5.2 Journal of galaxies with measured emission lines . . . . . . . . . . 170

5.3 Final Spectral classification . . . . . . . . . . . . . 179

5.4 Average properties of X-ray sources . . . . . . . . . . . . . . . 184

5.5 Luminous off-nuclear X-ray sources . . . . . . . . . . . . . . 188

5.6 Nuclear properties of nearby galaxies . . . . . . . . . . . . . . . 191

5.7 Internal extinction estimates f. . . . . . . . . . . . . . . 204

5.8 Gaussian fits to the extended $\mathrm{H} \alpha \ldots \ldots$. . . . . . . . . . . . . . . . . . . . 


\section{Chapter 1}

\section{Introduction}

\subsection{How common are AGN?}

Just how widespread nuclear activity is amongst galaxies is not well known. Are Active Galactic Nuclei (AGN) restricted to a small fraction of galaxies, or is it possible, if we look hard enough, to find signatures of weak nuclear activity in all galactic nuclei? Our understanding of the nature of nuclear activity will depend on whether AGN are found to be rare or widespread phenomena.

The luminosity distribution for quasars and luminous Seyfert galaxies in the nearby universe is well established. Their high nuclear luminosity makes it easy to distinguish between AGN and normal nuclei. Meurs \& Wilson (1984) compared the luminosity distribution of Markarian Seyferts with that of field galaxies and found that active galaxies comprised $\sim 1 \%$ of all galaxies in the $-19 \geq M_{B} \geq-24$ range. This picture has since changed with the results from more sensitive surveys showing that the fraction of active galaxies could be as high as $\sim 40 \%$, with $\sim 10 \%$ being Seyfert galaxies (Ho, Filippenko \& Sargent 1997d).

Searching for and studying low-luminosity AGN becomes more and more difficult as the emission from stars rivals or out-shines the active nucleus. Pioneering work on the detection of low-luminosity nuclear activity in galaxies considered otherwise normal was done by Peimbert \& Torres-Peimbert (1981) on M 81 and Rose \& Searle (1982) on M 51 who showed that Seyfert characteristics with mild absolute strengths can be seen in these galactic nuclei. 


\subsubsection{Recognising AGN}

By definition, (Type-1) Seyfert galaxies are objects whose nuclei show strong, broad emission lines which come from a bright semi-stellar nucleus (Khachikian \& Weedman 1974). However, AGN are also strong radio and X-ray emitters, so surveys conducted at these frequencies can discover the presence of active nuclei that have been overlooked in optical searches (Phillips, Charles \& Baldwin 1983). The presence of active nuclei in galaxies can be best assessed, therefore, with multiwavelength data.

The recognition of the presence of Low Ionization Nuclear Emission-line Regions (LINERs) in the nuclei of many nearby galaxies has opened the possibility that nuclear activity can have line properties that depart from those seen in classical Seyfert galaxies. This diversity in line features could be due to the different physical conditions that prevail in galactic nuclei.

\section{Are LINERs active nuclei?}

In 1980 Heckman identified a new class of emitting regions in galactic nuclei that did not resemble the spectra of HII regions or classic Seyfert galaxies, and called them LINERs. The main properties of this new class were an optical spectrum dominated by emissionlines from low ionization species and a preference for early-type spirals as host galaxies. Although normally regarded as the faintest expression of nuclear activity, the nature of LINERs is still an unsolved puzzle. The optical spectroscopic characteristics of LINERs have been successfully explained by a variety of models: a small ionization parameter (U) in a model with a power-law photoionizing source (Ferland \& Netzer 1983; Ho, Filippenko \& Sargent 1993); ionization by shocks (Dopita et al. 1997; Dopita et al. 1996); photoionization by extremely hot ( $\mathrm{T} \sim 100,000^{\circ} \mathrm{K}$ ), metal-rich stars or WARMERs (Terlevich \& Melnick 1985); ionization by supernova-driven outflows (Moorwood et al. 1996); and so on. This proliferation reflects our poor understanding of the LINER phenomenon.

The most probable explanation is that LINERs are indeed an heterogeneous class of objects. The detection of broad $\mathrm{H} \alpha$ wings in a significant fraction of LINERs (see next section and Ho 1998) suggests that at least some of these objects are genuine AGN, while recent UV observations show the signature of massive stars, indicating a stellar origin for the UV continuum in some cases (Maoz et al. 1998). 
Ho, Filippenko \& Sargent (1995a; 1997a; 1997c; 1997d) have recently reported on the results from the nuclear spectroscopy of a magnitude-limited sample $(B \lesssim 12.5)$ of $\sim 500$ galaxies in the northern hemisphere. Based on intensity ratios of several narrow emission lines they classified the galaxies as Seyferts, (pure) LINERs, Transition objects (a LINER nucleus contaminated by HII region emission) and HII nuclei. After careful subtraction of the stellar light they also searched amongst the AGN (collectively Seyfert, LINER and Transition nuclei) for broad $\mathrm{H} \alpha$ emission. Their main results are listed below:

- Emission-line nuclei are detected in $86 \%$ of the galaxies in the sample and they divide nearly equally between HII nuclei and AGN (with Seyferts, pure LINERs and Transition objects detected in $\sim 10 \%, 19 \%$ and $13 \%$ of the total sample, respectively).

- Consistent with previous studies (Heckman 1980; Keel 1983; Stauffer 1982), LINERs are found in $\sim 1 / 3$ of all galaxies and, under the assumption that they are genuine AGN, they represent $\sim 3 / 4$ of the AGN population.

- Broad $\mathrm{H} \alpha$ is detected in $\sim 10 \%$ of the galaxies in the sample (about $20 \%$ of the AGN population), about half of which have LINER nuclei.

\section{Detection of $\mathrm{AGN}$ at radio and X-ray wavelengths}

The continuum emission of all AGN covers an enormously broad range of frequencies, from radio waves $\left(\lesssim 10^{6} \mathrm{~Hz}\right)$ to $\gamma$-rays $\left(z 10^{23} \mathrm{~Hz}\right)$. Based on the relative strength of the radio emission, an active nucleus can be classified as a 'radio-quiet' or 'radio-loud' AGN. The closest example of a radio-loud object is more than $20 \mathrm{Mpc}$ away (M 87, a massive elliptical galaxy located at the centre of the Virgo cluster).

The multiwavelength nature of AGN is one of their most important properties. The optical identification of extragalactic X-ray and radio sources is a successful tool in detecting previously unknown AGN. The detection of compact nuclear X-ray and radio sources in galaxy nuclei is particularly useful when studying low level AGN activity. Their detection gives strong support to classifying objects that do not appear to be active galaxies at optical wavelengths (see, for example, the discussion in Filippenko 1992 and chapter 4 of this thesis). 


\subsection{Relationship with host galaxy properties}

The host galaxies of AGN hold clues to several important questions about nuclear activity. Do all galaxies have the same probability of harbouring an AGN? Or is this probability a function of some of the host galaxy properties (morphology, luminosity, environment)? Do the central engines correlate with any of these properties? Under the massive Black Hole $(\mathrm{BH})$ paradigm, we would like to understand how such BHs form and how this is connected with the galaxy formation process.

\subsubsection{Correlation with host galaxy luminosity}

In their study of a magnitude-limited sample of nearby galaxies Huchra \& Burg (1992) compared the absolute magnitude of the total 'integrated' galaxy with the nuclear absolute magnitude obtained through a 'small-aperture'. They found a close to linear correlation between the two quantities. They noted, however, that the procedure was not ideal since it did not properly remove the stellar component from the bulge, disk, and nucleus itself.

In their optical and infrared study of a X-ray-selected sample of Seyfert 1 galaxies, Kotilainen \& Ward (1994) fitted the galactic radial profiles with three components: an unresolved nucleus, a bulge following the $r^{1 / 4}$ law, and an exponential disk. Comparing the absolute magnitudes of the nuclear and host galaxy obtained in this way they found that the luminosity of the active nucleus was an increasing function of the luminosity of the parent galaxy. They argued that the lack of detection of faint nuclei in bright galaxies was not due to selection effects because the sample had been defined based on the X-ray properties of the galaxies. However, this argument applies only if there is no correlation between the X-ray luminosity of the Seyfert nuclei and the luminosity of the galaxy.

The first quantitative studies of the properties of the parent galaxies of quasars also showed a correlation between the optical luminosity of the active nucleus and the absolute magnitude of the host galaxy (Gehren et al. 1984; Hutchings, Crampton \& Campbell 1984). From a study of 17 objects Gehren et al. (1984) found that the luminosity of the quasar nuclei may be loosely correlated with the absolute magnitude of their host galaxies'. Using a larger sample of 78 nearby quasars Hutchings, Crampton \&: Campbell (1984) found a tight correlation between the absolute magnitude of the nuclei and the parent galaxy. They noticed that while selection effects may have prevented the discovery of low-luminosity nuclei in luminous galaxies, there was no explanation for the non-detection of luminous nuclei in less luminous hosts. They therefore considered that the observed 
correlation could be, in fact, an upper bound.

The suspicions of Hutchings, Crampton \& Campbell (1984) have been confirmed as more, and better quality, data have become available. McLeod (1997) has determined nuclear and host galaxy near-IR magnitudes for a sample of low redshift quasars. He found that for a given host luminosity there is a maximum nuclear luminosity which is an increasing function of the luminosity of the parent galaxy. This suggests that for each galaxy size there is a maximum possible BH mass, and so a maximum AGN luminosity.

Results on the demography of AGN and their connection with the parent galaxy normally come from the analysis of magnitude-limited samples. In these samples the population of small galaxies is ill-represented, biasing our understanding towards big, luminous galaxies.

Hence, it is important to establish:

- if nuclear activity is really a widespread phenomenon present in all galaxies;

- if the level of activity has a real cut-off towards the faint end of the galaxy distribution;

- if the correlation between host and nuclear luminosity observed in luminous objects is also seen for the fainter galaxies.

Ideally, we would like to have a large, unbiased sample of galaxies to determine a bivariate luminosity function for AGN and so untangle the connection between host and nucleus.

\subsubsection{Correlation with host galaxy morphology type}

A 'simple' picture of the relationship between the nuclear radio loudness of active galaxies and the morphological type of their hosts has been inferred from the study of nearby AGN: radio-loud AGN are hosted by ellipticals while radio-quiet are hosted by spirals (see, for example, the review paper by Lawrence 1987 and references therein).

New evidence has started to change this view, however. The study of luminous quasars at moderate redshift $(0.1<z<0.35)$ by McLure et al. (1998) shows that the host galaxies of all radio-loud and nearly all radio-quiet objects are massive elliptical galaxies. The analysis suggests that the probability of a radio-quiet AGN having an elliptical host is an increasing function of the quasar luminosity. Although this result shows that a direct link 
between radio loudness and host morphology no longer holds for high luminosity quasars, it is in excellent agreement with the BH-bulge mass relationship found by Magorrian et al. (1998) (see discussion below).

Surveys of local galaxies also show that most active nuclei reside in early-type bulgedominated galaxies while nuclei with HII region characteristics prefer late-type diskdominated hosts (see, for example, Ho, Filippenko \& Sargent 1997d and the review paper by Lawrence 1987). The fact that LINERs and Seyfert galaxies show an indistinguishable distribution of host galaxy types gives support to the classification of LINERs as genuine AGN.

\subsection{Cosmological context}

An important part of the current understanding of nuclei activity and its connection with host galaxies comes from high redshift studies. The study of the number of quasars versus look-back time shows an evolution of the luminosity function $\phi\left(L_{\nu}, z\right)$ (the number of sources with luminosity $L_{\nu}$ per unit volume at redshift $z$ ) with redshift. This is not the evolution of individual objects, but a statistical property of the quasar population, and is an oversimplification of the actual changes occurring at the individual level. The evolution of the luminosity function with redshift shows a decrease in the luminosity of the quasar population from $z \sim 2$ to the present (Boyle et al. 1991).

This decline with cosmic time can, in principle, be explained as 'density evolution' and/or 'luminosity evolution' (which correspond, respectively, to vertical and horizontal shifts in a $L_{\nu}$ versus $\phi\left(L_{\nu}\right)$ plot). The determination of the luminosity function at different redshifts for optical and X-ray selected samples of quasars has shown that the changing population is consistent with pure luminosity evolution (Boyle et al. 1991; Boyle, Wilkes \& Elvis 1997).

Luminosity evolution can be explained by two different scenarios. In one case most galaxies harbour massive BHs and have short lived active phases, with the luminosity of each phase decreasing with time. In the second case only a small fraction of all galaxies have long-lived active nuclei and the luminosity of this population has been fading out since $z \sim 2$.

These two scenarios have very different predictions for the local population of active and dormant AGN. The first case implies that many, maybe all, galaxies in the local universe harbour a dormant or weakly active BH in their nuclei. In the second case the fading 
population of quasars could be seen in the local universe as low redshift quasars and Seyfert nuclei. The population of high-redshift quasars could then be directly compared with the local space density of AGN.

Soltan (1982) and Chokshi \& Turner (1992) showed that the total, integrated luminosity of high-redshift quasars implies a local mass density of $\mathrm{BHs}$ of $(1.4-2.2) \times 10^{5} \mathrm{M}_{\odot} \mathrm{Mpc}^{-3}$, assuming a standard BH accretion model and a quasar radiative efficiency of 0.1 . This implies that every galaxy could harbour a $\sim 10^{7} M_{\odot}$ BH. If only luminous galaxies $(L z$ $L^{*}$ ) are assumed to host an AGN, then the BH mass for those objects increases by a factor $\sim 3$.

Using a model that assumes the existence of many short-lived quasars, active during the first phase of the formation of a galaxy, Haehnelt \& Rees (1993) can reproduce the luminosity function for quasars at intermediate and high redshifts. As a consequence, remnant $\mathrm{BHs}$ would be expected in any moderately bright galaxy which has a potential well sufficiently deep to retain gas.

\subsection{Dormant black holes}

The great majority of galaxies in the nearby universe seem to be inactive or to have only low-luminosity active nuclei. If most galaxies harbour massive central BHs then something must prevent them from becoming as active as Seyfert galaxies. Lack of fuel or an extremely inefficient accretion processes could be the answer. The detection of dormant $\mathrm{BHs}$ in normal galaxies is an important piece in the nuclear activity puzzle.

In the last few years much effort has been invested in trying to establish the ubiquity of Massive Dark Objects (MDOs) in the nuclei of galaxies. The search for MDOs requires extremely high spatial resolution in order to determine whether the motion of gas and stars is consistent with a strong central potential well. So far, there are claims of detected MDOs in about a dozen galaxies. Recent review papers of the field can be found in Kormendy \& Richstone (1995), Van der Marel (1998), Richstone et al. (1998), and Ford et al. (1998).

Methods based on stellar dynamics show that a steep increase in the velocity dispersion towards the centre of the galaxy is a strong indicator of the presence of a MDO. One of the most spectacular results in this area comes from the IR proper motion study of stars in the nuclear region of our own Milky Way. Genzel et al. (1997) and Ghez et al. (1998) 
find that the data are consistent with a central MDO with a mass of $2 \times 10^{6} \mathrm{M}_{\odot}$ within $0.006 \mathrm{pc}$ of the nucleus.

Methods based on gas dynamics can be more difficult to interpret since gas responds to other forces apart from gravity, such as shocks, radiation pressure, and magnetic fields. However, they can also achieve astonishing resolutions $\left(\theta \sim 10^{-4} \operatorname{arcsecs}\right)$. VLBA studies of water masers in NGC 4258 have given some of the most compelling evidence yet of a massive object in a nearby galaxy (Miyoshi et al. 1995).

A few systematic searches for MDOs have been conducted. A careful dynamical search carried out by Kormendy \& Richstone (1995) yielded a $20 \%$ detection rate within a small sample of nearby (E-Sb) galaxies. This result should be regarded as a lower limit. Magorrian et al. (1998) studied 36 elliptical galaxies using HST photometry and ground-based spectroscopy. The modelling of the data shows that in all but six galaxies a MDO is required at $95 \%$ confidence level.

The evidence strongly favors a picture where a large fraction of all galaxies harbour a MDO which could be in the form of a massive BH. The observations also show that a correlation might exist between the mass of the central object and the mass of the spheroidal component of the galaxy (Richstone et al. 1998), with the mass of the MDO being a few thousandths the mass of the galaxy bulge. This correlation could hold important clues to how MDOs and galaxies form.

Although the BH scenario is not the only plausible explanation for the massive objects found in galaxy nuclei, it is the most compelling one. Direct proof of the presence of a BH would have to come from the detection of signatures of relativistic motions in the vicinity of the hole. In this regard, the detection of gravitationally redshifted iron K $\alpha$ lines at 6.4 $\mathrm{keV}$ in active galaxies is the best evidence so far for the presence of BHs in galactic nuclei (Tanaka et al. 1995; Nandra et al. 1997).

In summary, results from the search of MDOs are consistent, within the errors, with our common knowledge of $\mathrm{BH}$ demography, masses and evolution. Also, the MDO-mass / bulge-mass correlation and the AGN / host luminosity relationship seem to show a similar trend, where the biggest galaxies can harbour the most massive BHs. 


\subsection{A new project: the multiwavelength study of a volume- limited sample of galaxies}

\subsubsection{Motivation}

Most studies of AGN and their host galaxies have been restricted to large, luminous objects. Low luminosity galaxies are under-represented in magnitude limited samples and therefore little is known about the occurrence of nuclear activity in them. The few examples of known low luminosity active nuclei are hosted by relatively small galaxies, like the Seyfert 1 nucleus in NGC 4395 and the Seyfert 2 nucleus in G1200-2038, where both galaxies have $M_{B} \sim-18$ (see chapter 4 in this thesis and Kunth, Sargent \& Bothun 1987). To establish if even smaller galaxies can host an active nucleus a more representative sample of objects must be studied.

\subsubsection{The sample}

To study the occurrence of nuclear activity in galaxies to the lowest possible level, Professor A. Lawrence and Dr. R. A. Johnson defined a distance-limited sample of galaxies. The sample contains the nearest examples of various morphological types of galaxies, and a representative range of intrinsic luminosities (Johnson 1997).

The starting point for the selection criteria was the Kraan-Korteweg \& Tamman Catalogue (Kraan-Korteweg \& Tammann 1979; Kraan-Korteweg 1986), with all galaxies with V < $500 \mathrm{~km} / \mathrm{s}$, giving a distance-complete sample within $d=0.35 \times d_{\mathrm{Virgo}}$ (after applying a virgocentric flow model with an infall velocity of the Local Group equal to $220 \mathrm{~km} \mathrm{~s}^{-1}$ ). With $d_{\text {Virgo }}=21.5 \mathrm{Mpc}$, the sample contains all nearby galaxies within $7 \mathrm{Mpc}$. The catalogue is not complete for intrinsically faint galaxies without velocity information, but it probably includes all galaxies down to $M_{B} \sim-13$. Comparing the distribution of absolute magnitudes of the galaxies in the KKT with the galaxies in the Revised Shapley Ames (magnitude-limited) catalogue (Sandage \& Tammann 1981) it is found that the RSA sample of galaxies presents a strong peak around $M_{B}=-21$ and has less than $10 \%$ of the objects below $M_{B}=-18$, while the KKT sample shows a broad distribution with a maximum around $M_{B}=-16$ and a significant number of galaxies down to magnitude $M_{B}=-13$.

The observations in the volume-limited sample were restricted to objects with $\delta>-35^{\circ}$. Because the aim was to observe objects with well-formed nuclei, galaxies classified as Sdm, 
$\mathrm{Sm}$ and $\mathrm{Irr}$ were removed from the sample. This gave a basic subsample of 46 galaxies (28 spirals, $5 \mathrm{E} / \mathrm{S} 0,12$ dwarf $\mathrm{E}$ and 1 peculiar). The assumed distances for the galaxies are based on the observed recession velocities and a Virgo flow model with an infall velocity of $220 \mathrm{~km} \mathrm{~s}^{-1}$ for the Galaxy (Kraan-Korteweg 1986). The subsample and its properties are listed in table 1.1.

To carry out a comprehensive study of the nuclear regions of the volume-limited sample, a multiwavelength search project was started in 1993, collecting radio, optical, near-IR and X-Ray broad band data. The analysis of these images gave a list of targets regarded as candidates for nuclear activity. Long-slit spectroscopy was carried out for most of these sources. Table 1.1 lists the available data.

\subsection{Thesis outline}

The project was started before, and will continue after, this thesis. Johnson (1997) has already reported on the main results from the analysis of the broad band IR and optical imaging. The research reported here has concentrated mainly on the acquisition, reduction and analysis of high resolution X-ray imaging and optical spectroscopy. The contents of this thesis are outlined below.

The rest of this introduction reviews the present knowledge on the nature of individual $\mathrm{X}$-ray sources and the X-ray emission from nearby galaxies.

Chapters 2 and 3 describe the high resolution X-ray observations and optical spectroscopy, respectively, of the galaxies in the sample. The first part of each chapter describes the acquisition and reduction of data. The second part of each chapter reports on the main results from the observations. Chapter 4 is devoted to the study of NGC 4395, an extremely low-luminosity Seyfert 1 galaxy where variability has been observed for the first time. Chapter 5 analyses the results from the X-ray and spectroscopic data for the sample as a whole and compares them with models of nuclear star formation and AGN properties. Finally, chapter 6 summarises the major results and conclusions.

\subsection{Sources of X-ray emission}

In this section the various components that might contribute to galactic $\mathrm{X}$-ray emission will be discussed. 
There are four main types of galactic X-ray sources: stars, cataclysmic variables (CVs), high mass and low mass X-ray binaries (XRBs), and Supernova Remnants (SNRs). A brief review of the current knowledge on the characteristics of these sources is given below, with an emphasis on the components that dominate galactic X-ray emission. For a description of the physical X-ray emission mechanisms see appendix A. The main reference sources in the next sections are Schmitt (1990) and Linsky (1990) for stars, Cordova (1995) for CVs, Lozinskaya (1992) for SNRs, and Van Paradijs \& McClintock (1995) and White, Nagase \& Parmar (1995) for XRBs.

\subsubsection{Stars}

The study of late-type stars in the Galaxy shows that typical X-ray luminosities in the Einstein band-pass are between $10^{27}$ and $10^{30} \mathrm{ergs} \mathrm{s}^{-1}$. It is believed that the X-ray emission is linked to the stellar dynamo activity. Early-type stars are stronger emitters with $L_{X} \sim 10^{32}-10^{33}$ ergs s$^{-1}$. The physical mechanisms responsible for the coronal emission are thought to be shocks generated in winds (in massive stars) and dissipation of energy from acoustic waves generated in the convection zone (in dwarfs).

\subsubsection{Cataclysmic variables}

CVs are binary systems in which a low-mass star transfers mass to its compact white dwarf companion. The binaries have to be close enough for the low mass star to fill its Roche lobe so that mass transfer and accretion can take place. CVs are important X-ray emitters with luminosities in the soft band pass of $10^{30}-10^{32} \mathrm{ergs} \mathrm{s}^{-1}$. The optical emission from CVs originates from energy internally generated in the accretion disk.

\subsubsection{Supernova remnants}

Supernova (SN) explosions have a profound effect on their surroundings: the interstellar medium is enriched with heavy elements and vast amounts of energy are pumped into the interstellar medium. Two different processes are responsible for the events.

Type Ib and Type II SN are the result of the gravitational collapse of a massive young star $\left(M z 10 M_{\odot}\right)$. As these stars exhaust their internal fuel and radiative pressure no longer supports their own weight, the internal layers collapse to form a compact remnant (a neutron star or pulsar) while the outer layers bounce outwards and are ejected into the 
interstellar medium.

Type Ia SN are believed to correspond to the explosion of white dwarfs in binary systems which are accreting matter from their companion. When the white dwarf reaches the Chandrasekhar limit $\left(M \sim 1.4 M_{\odot}\right)$ the star ignites. All the stellar matter is ejected into the surrounding medium and no compact remnant is left.

After the initial SN explosion the ejecta expand and interact with the interstellar medium and become known as a supernova remnant (SNR). The evolution of a SNR can be separated into the three major stages.

- 0 - 100 years: The ejecta expand freely with velocities of about $10,000 \mathrm{~km} \mathrm{~s}^{-1}$, sweeping up the low density interstellar medium. One shock is formed at the leading edge of the ejecta and a reverse shock is formed at the back.

- 100 - 1000 years: The shell of expanding material slows as the mass of swept-up material becomes larger than the total mass of the ejecta. In the interior of the shell a bubble of hot tenuous gas is formed with temperatures of $\sim 10^{8} \mathrm{~K}$.

- $10^{3}-10^{5}$ years: When the shocked material cools to about $10^{5} \mathrm{~K}$, the shell collapses into a dense layer and recombination between electrons and ions is possible. At this point the remnant becomes radiative. During this phase most of the internal energy is radiated away.

Supernova remnants are strong radio, optical and X-ray sources. The optical emission comes from collisionally excited lines of metal ions at temperatures below $10^{5} \mathrm{~K}$ during the final stage in the evolution of the remnant. Radio and X-ray emission originate from hotter material at the interior of the shell and predominate in the earlier stages. The $\mathrm{X}$-ray emission is thermal: free-free radiation comes from the shocked material that has been compressed and heated by the expanding ejecta. The temperature of this gas can be as high as $10 \mathrm{keV}$. Synchrotron radiation from relativistic electrons moving through moderate magnetic fields are responsible for the radio emission.

As will be discussed in more detail later in chapter 5, the continuous deposition of kinetic energy by SNRs during violent episodes of star formation can form giant bubbles of hot low-density expanding gas. The most dramatic examples of this process can be seen in starburst galaxies with X-ray extended emission in the form of 'winds' or 'plumes' well below and above the galactic disks. 


\subsubsection{X-ray binaries}

Binary systems where the most compact member, a neutron star or a black hole, accretes matter from the companion are strong X-ray sources. The X-ray characteristics of the system depend on the nature of the compact star, the strength of the magnetic field in the case of neutron stars, and the regime of matter accretion from the companion.

In neutron stars with strong magnetic fields the accretion flow will be disturbed and funelled onto the magnetic poles on the surface of the star. X-ray emission from such a system will come mainly from the magnetic caps. If the magnetic pole does not coincide with the rotating axis of the star a light house effect occurs and emission is observed whenever the active zone includes the line of sight (i.e. a pulsar). For weak magnetic fields the matter will form an accretion disk around the star and the X-ray emission will come from the inner parts of the disk and from the surface of the star. A black hole will also form an accretion disk which will be the source of radiation.

All X-ray binaries are variable, showing bursts or flares of activity, many of them in recurrent patterns. Variability is thought to be a result of instabilities in the accretion flow and/or a modulation of the emission due to the orbital period or the presence of a pulsar. The so-called transient objects show increases in luminosity by 3 or 4 orders of magnitude and their peak luminosities can reach as high as $\sim 10^{39} \mathrm{ergs} \mathrm{s}^{-1}$.

X-ray binaries are normally classified into two broad categories: low mass X-ray binaries (LMXRB), where the donor star is a low mass $\left(M \lesssim 1 M_{\odot}\right)$ main sequence star, and high mass X-ray binaries (HMXRB), where the companion star is a young massive star $\left(M \gtrsim 10 M_{\odot}\right)$ which dominates the optical emission of the system.

The massive stars in HMXRB have extremely strong stellar winds $\left(10^{-10}-10^{-8} M_{\odot}\right.$ per year) which are captured by the strong potential well of the compact object and then accreted. The accretion can be free infall because stellar winds do not carry a significant amount of angular momentum. In contrast, the donor star in a LMXRB system must be close enough to the compact object to fill its critical Roche lobe for transfer of matter to occur. The angular momentum carried by the matter will cause it to circle around the neutron star or black hole, forming an accretion disk under the effect of viscosity. The main source of optical emission in a LMXRB is the reprocessing of X-rays in the accretion disk. Examples of HMXRB are Cen X-3, Cyg X-1, LMC X-3; LMXRB examples include Sco X-1, Her X-1, Cyg X-2. 
HMXRBs are normally sub-divided into two groups: supergiants and Be systems. In the first group the donor is a supergiant $O B$ star with a fairly symmetric and constant stellar wind. In the second group the donor is a Oe or Be-type star, characterised by Balmer emission lines that originate in circumstellar material and by asymmetric and highly variable winds. Since the mass loss rate of the donor star governs the accretion rate of the compact companion in a HMXRB it is not a surprise that the X-ray emission from supergiant and Be systems show very different properties. Be systems are highly variable, with quiescent periods followed by outbursts, reflecting the change in the accretion rate. Supergiant systems have a persistent $\mathrm{X}$-ray luminosity comparable to that seen in Be binaries during their active phases.

The observed X-ray luminosities of LMXRBs can be as high as the Eddington limit for a $\sim 1 M_{\odot}$ compact object $\left(L_{X} \sim 10^{38} \mathrm{ergs} \mathrm{s}^{-1}\right)$. However, HMXRBs maximum luminosities rarely reach this limit and the quiescent luminosities of Be HMXRBs can be as low as $\lesssim 10^{30}$ ergs s$^{-1}$.

\subsection{X-rays from the nearest galaxies}

Most of our detailed knowledge of X-ray emission comes from the study of very nearby galaxies. Local Group galaxies allow us to study the distribution of X-ray sources throughout the galaxy and examine their correlation with other galactic structures. The best studied nearby galaxies are M31, which is the biggest member of the Local Group and has a similar morphology to our own Galaxy, and the Magellanic Clouds, satellite galaxies of the Milky Way.

\subsubsection{NGC 224 (M 31)}

Einstein and ROSAT observations of M 31 have detected over 100 individual sources with luminosities $5 \times 10^{36}<L_{x}<10^{38} \mathrm{ergs} \mathrm{s}^{-1}$. No significant extended emission has been found.

The sources are associated with two components, the disk and the bulge, and are strongly concentrated towards the centre. The bulge and disk components each account for about half of the total X-ray emission. There is no significant difference between the luminosity distribution of the disk and bulge sources, which both follow a power law fit with index $\sim 0.4$. 
Within the central 5 ' the bulge has an X-ray luminosity of $\sim 1.4 \times 10^{39} \mathrm{ergs} \mathrm{s}^{-1}$ of which approximately $75 \%$ is accounted for by observed discrete sources. The remaining emission is consistent with extrapolating the observed luminosity distribution to objects below the detection threshold. Ginga observations have shown that this emission is consistent with a population of LMXRB.

\subsubsection{The Magellanic Clouds}

The Small and Large Magellanic Clouds (the LMC and SMC) contain some of the most remarkable X-ray sources in the Local Group. The large number of detected SNRs and the highly luminous XRBs are believed to be caused in part by the observed low metalicities.

In the LMC $\sim 40$ SNRs have been confirmed and $\sim 20$ X-ray sources are SNR candidates (Filipovic 1998). These numbers are much larger than expected by scaling the number of SNRs observed in the Milky Way by the mass ratio of the two galaxies (Helfand 1984). The luminosities of some of these objects can be quite large with most of the remnants in the $10^{36}-10^{38}$ ergs s$^{-1}$ luminosity range (Long, Helfand \& Grabelsky 1981).

The most luminous sources in the LMC are XRBs, normally high mass systems associated with the Population I component of the galaxy. The luminosities of these sources are normally above $10^{38} \mathrm{ergs} \mathrm{s}^{-1}$ with a mean (maximum) luminosity of $\left\langle\log L_{X}\right\rangle=38.4 \pm 0.5$ $\operatorname{ergs~s}^{-1}$, compared with a mean luminosity for Galactic HMXRBs of $\left\langle\log L_{X}\right\rangle=36.7 \pm 0.9$ $\operatorname{ergs~s}^{-1}$ (Van Paradijs \& McClintock 1995). LMXRBs are very rare in the LMC suggesting the lack of an old population in the galaxy. However, this explanation is not completely satisfactory because old population stars like RR Lyra are indeed observed (Cowley et al. 1998). 


\begin{tabular}{|c|c|c|c|c|c|c|c|c|c|}
\hline \multirow[b]{2}{*}{ Galaxy } & \multirow[b]{2}{*}{ Position (2000) } & \multirow[b]{2}{*}{$-M_{B}$} & \multirow[b]{2}{*}{ Morph } & \multirow[b]{2}{*}{ D $(\mathrm{Mpc})$} & \multicolumn{4}{|c|}{ Broad Band Observations } & \multirow{2}{*}{$\begin{array}{l}\text { Nuclear } \\
\text { Spect. }\end{array}$} \\
\hline & & & & & Radio & Near-IR & Optical & X-ray & \\
\hline NGC 147 & $0030+48 \quad 14$ & 13.71 & dE5 & 0.65 & $\sqrt{ }$ & $\sqrt{ }$ & $\sqrt{ }$ & $\sqrt{ }$ & $\sqrt{ }$ \\
\hline ANDIII & $0033+3614$ & 10.17 & $\mathrm{dE}$ & 0.65 & $\sqrt{ }$ & & $\sqrt{ }$ & & \\
\hline NGC 185 & $0036+4804$ & 13.94 & dE3 & 0.65 & $\sqrt{ }$ & $\sqrt{ }$ & $\sqrt{ }$ & $\sqrt{ }$ & $\sqrt{ }$ \\
\hline NGC 205-M 110 & $0038+4125$ & 15.24 & S0/E5 & 0.65 & $\sqrt{ }$ & $\sqrt{ }$ & $\sqrt{ }$ & $\sqrt{ }$ & $\sqrt{ }$ \\
\hline NGC 221-M 32 & $0040+4036$ & 15.06 & E2 & 0.65 & $\sqrt{ }$ & $\sqrt{ }$ & $\sqrt{ }$ & $\sqrt{ }$ & $\sqrt{ }$ \\
\hline NGC 224-M 31 & $0040+4060$ & 19.69 & $\mathrm{Sb}$ & 0.65 & $\sqrt{ }$ & $\sqrt{ }$ & $\sqrt{ }$ & $\sqrt{ }$ & $\sqrt{ }$ \\
\hline ANDI & $0043+3744$ & 10.17 & dE3 & 0.65 & $\sqrt{ }$ & $\sqrt{ }$ & $\sqrt{ }$ & & \\
\hline $\mathrm{NGC} 247$ & $0045-2102$ & 18.32 & $\mathrm{Sc}(\mathrm{s})$ & 3.69 & $\sqrt{ }$ & & $\sqrt{ }$ & $\sqrt{ }$ & $\sqrt{ }$ \\
\hline $\mathrm{NGC} 253$ & $0045-2534$ & 20.26 & $\mathrm{Sc}(\mathrm{s})$ & 4.77 & $\sqrt{ }$ & $\sqrt{ }$ & & $\sqrt{ }$ & \\
\hline SCULPTOR & $0058-3358$ & 10.38 & dE3 & - & $\sqrt{ }$ & & & & \\
\hline NGC 404 & $0107+3527$ & 17.07 & S0 & 4.34 & $\sqrt{ }$ & $\sqrt{ }$ & $\sqrt{ }$ & $\sqrt{ }$ & $\sqrt{ }$ \\
\hline AND II & $0114+3309$ & 10.17 & $\mathrm{dE}$ & 0.65 & $\sqrt{ }$ & $\sqrt{ }$ & $\sqrt{ }$ & & \\
\hline NGC 598-M 33 & $0131+3024$ & 18.43 & $\mathrm{Sc}(\mathrm{s})$ & 0.87 & $\sqrt{ }$ & $\sqrt{ }$ & & $\sqrt{ }$ & $\sqrt{ }$ \\
\hline MAFFEI I & $0233+5926$ & 13.87 & E3 & 5.43 & $\sqrt{ }$ & $\sqrt{ }$ & $\sqrt{ }$ & & $\sqrt{ }$ \\
\hline FORNAX & $0238-3444$ & 12.64 & $\mathrm{dE} 0$ & 0.22 & $\sqrt{ }$ & & & & \\
\hline MAFFEI II & $0238+5923$ & 8.08 & $\mathrm{Sbc}$ & 5.21 & $\sqrt{ }$ & $\checkmark$ & $\sqrt{ }$ & & $\sqrt{ }$ \\
\hline IC 342 & $0342+6756$ & 19.50 & $\mathrm{~S}(\mathrm{~B}) \mathrm{cd}(\mathrm{rs})$ & 6.08 & $\sqrt{ }$ & $\sqrt{ }$ & $\sqrt{ }$ & $\sqrt{ }$ & $\sqrt{ }$ \\
\hline NGC 1560 & $0427+7146$ & 15.95 & $\mathrm{Sd}(\mathrm{s})$ & 4.34 & $\sqrt{ }$ & & & $\sqrt{ }$ & $\sqrt{ }$ \\
\hline NGC 2366 & $0724+6919$ & 17.53 & $\mathrm{SBm}$ & 6.29 & $\sqrt{ }$ & $\checkmark$ & $\sqrt{ }$ & $\sqrt{ }$ & $\sqrt{ }$ \\
\hline NGC 2403 & $0732+6543$ & 20.25 & $\mathrm{Sc}(\mathrm{s})$ & 6.73 & $\sqrt{ }$ & $\sqrt{ }$ & $\sqrt{ }$ & $\sqrt{ }$ & $\sqrt{ }$ \\
\hline NGC 2976 & $0943+6809$ & 16.98 & $\mathrm{Sd}$ & 3.69 & $\sqrt{ }$ & $\sqrt{ }$ & $\sqrt{ }$ & $\sqrt{ }$ & $\sqrt{ }$ \\
\hline A $0951+68$ & $0951+6850$ & 11.21 & $\mathrm{dE}$ & 1.52 & $\sqrt{ }$ & $\sqrt{ }$ & $\sqrt{ }$ & $\sqrt{ }$ & $\sqrt{ }$ \\
\hline NGC 3031-M 81 & $0952+6918$ & 19.22 & $\mathrm{Sb}(\mathrm{r})$ & 2.60 & $\sqrt{ }$ & $\sqrt{ }$ & $\sqrt{ }$ & $\sqrt{ }$ & \\
\hline LEO I & $1006+1233$ & 10.87 & dE3 & 0.22 & $\omega$ & $\checkmark$ & & & \\
\hline LEOB & $1111+2226$ & 8.98 & dE0 & 0.22 & $\omega$ & $\sqrt{ }$ & & $\sqrt{ }$ & $\sqrt{ }$ \\
\hline UGC 6456 & $1125+7916$ & 12.19 & peculiar & 2.39 & $\sqrt{ }$ & & $\sqrt{ }$ & $\sqrt{ }$ & $\sqrt{ }$ \\
\hline NGC 3738 & $1133+5448$ & 16.78 & $\mathrm{Sd}$ & 6.08 & $\omega$ & $\sqrt{ }$ & $\sqrt{ }$ & $\sqrt{ }$ & $\sqrt{ }$ \\
\hline NGC 4136 & $1207+3012$ & 17.63 & $\mathrm{Sc}(\mathrm{r})$ & 6.94 & $\sqrt{ }$ & $\checkmark$ & $\sqrt{ }$ & $\sqrt{ }$ & \\
\hline NGC 4144 & $1207+4644$ & 16.83 & $\mathrm{Scd}$ & 5.86 & $\omega$ & $\checkmark$ & $\sqrt{ }$ & $\sqrt{ }$ & $\sqrt{ }$ \\
\hline NGC 4150 & $1208+3041$ & 15.30 & $\mathrm{~S} 0 / \mathrm{a}$ & 3.47 & $\sqrt{ }$ & $\sqrt{ }$ & $\sqrt{ }$ & $\sqrt{ }$ & $\sqrt{ }$ \\
\hline NGC 4236 & $1214+6945$ & 17.50 & SBd & 3.26 & $\omega$ & $\checkmark$ & $\sqrt{ }$ & $\sqrt{ }$ & $\checkmark$ \\
\hline NGC 4244 & $1215+3805$ & 17.59 & Scd & 4.34 & $\sqrt{ }$ & $\sqrt{ }$ & $\sqrt{ }$ & $\sqrt{ }$ & \\
\hline UGC 7321 & $1215+2249$ & 14.82 & Scd & 5.43 & $\omega$ & & & & $\sqrt{ }$ \\
\hline NGC 4395 & $1223+3350$ & 17.89 & $\mathrm{Sd}$ & 5.21 & $\sqrt{ }$ & $\checkmark$ & & $\sqrt{ }$ & $\sqrt{ }$ \\
\hline NGC 4605 & $1238+6153$ & 17.80 & $\mathrm{Sc}(\mathrm{s})$ & 5.64 & $\omega$ & $\checkmark$ & $\sqrt{ }$ & $\sqrt{ }$ & $\sqrt{ }$ \\
\hline NGC 4736-M 94 & $1249+4124$ & 20.00 & RSab(s) & 6.08 & $\sqrt{ }$ & $\checkmark$ & $\sqrt{ }$ & $\sqrt{ }$ & $\sqrt{ }$ \\
\hline NGC 4826 & $1254+2157$ & 19.39 & $\mathrm{Sab}(\mathrm{s})$ & 5.64 & $\omega$ & $\checkmark$ & $\sqrt{ }$ & $\sqrt{ }$ & $\sqrt{ }$ \\
\hline NGC 5204 & $1328+5841$ & 17.39 & $\mathrm{Sd}$ & 6.73 & $\omega$ & $\sqrt{ }$ & $\sqrt{ }$ & $\sqrt{ }$ & $\sqrt{ }$ \\
\hline NGC 5238 & $1333+5152$ & 15.79 & $\mathrm{~S}(\mathrm{~B}) \mathrm{dm}$ & 6.73 & $\omega$ & $\sqrt{ }$ & $\sqrt{ }$ & $\sqrt{ }$ & $\sqrt{ }$ \\
\hline NGC 5236-M 83 & $1334-2937$ & 20.16 & $\mathrm{SBc}(\mathrm{s})$ & 5.43 & $\sqrt{ }$ & $\sqrt{ }$ & $\sqrt{ }$ & $\sqrt{ }$ & \\
\hline NGC 5457-M 101 & $1401+5436$ & 21.22 & $\mathrm{Sc}(\mathrm{s})$ & 7.60 & $\sqrt{ }$ & $\sqrt{ }$ & $\sqrt{ }$ & $\sqrt{ }$ & $\sqrt{ }$ \\
\hline URSA MINOR & $1508+6723$ & 7.40 & dE4 & - & & & $\sqrt{ }$ & & \\
\hline DRACO & $1719+5758$ & 7.00 & $\mathrm{dE} 0$ & - & & $\checkmark$ & $\sqrt{ }$ & & \\
\hline NGC 6503 & $1750+7010$ & 18.28 & $\mathrm{Sc}(\mathrm{s})$ & 6.94 & $\checkmark$ & $\sqrt{ }$ & $\sqrt{ }$ & $\checkmark$ & $\sqrt{ }$ \\
\hline NGC 6946 & $2034+5959$ & 19.66 & $\mathrm{Sc}(\mathrm{s})$ & 7.38 & $\sqrt{ }$ & $\sqrt{ }$ & $\sqrt{ }$ & $\sqrt{ }$ & $\sqrt{ }$ \\
\hline NGC 7793 & $2355-3252$ & 18.43 & $\mathrm{Sd}(\mathrm{s})$ & 4.12 & $\sqrt{ }$ & $\sqrt{ }$ & & & \\
\hline
\end{tabular}

Table 1.1: The sample of nearby galaxies. Data already available are indicated with a $\sqrt{ }$. Data that should become available in the near future are indicated with a $\omega$. 


\section{Chapter 2}

\section{High resolution X-ray imaging}

\subsection{The Roentgen Satellite}

ROSAT (ROentgen SATellite), an X-ray observatory named after the German physicist Wilhelm Roentgen, was developed through a cooperative program between Germany, the United States, and the United Kingdom. The satellite was launched in 1990 and during its first 6 months of activity performed an all-sky survey covering $\sim 98 \%$ of the sky. After this period ROSAT carried out pointed observations until April 1998, when a problem with the star tracker system began causing problems. These ended with accidental exposure to the Sun's radiation in September 1998, which caused irreversible damage to the instruments.

The pointed observations were coordinated on a yearly basis through the Announcement of Opportunity ( $\mathrm{AO}$ ) calls for proposals. The data belong to the principal investigator for the first year after the observations are released. After this period the data become public and any astronomer can obtain the observations from the archives.

ROSAT carried two co-aligned telescopes, the X-Ray Telescope (XRT) and the Wide Field Camera (an EUV telescope which had its own mirror and star sensor system). The XRT was used in conjunction with one of two focal plane instruments, the Position Sensitive Proportional Counter (PSPC) or the High Resolution Imager (HRI). The PSPC stopped being operational in September 1994. After this all pointed observations carried out by the XRT were done using the HRI.

The X-ray Mirror Assembly (XMA) on ROSAT had a focal length of 2.40 metres and was made from 4 nested mirrors each being a paraboloid-hyperboloid pair. All the mirrors were made of zerodur, a ceramic with an almost negligible thermal coefficient, and coated 


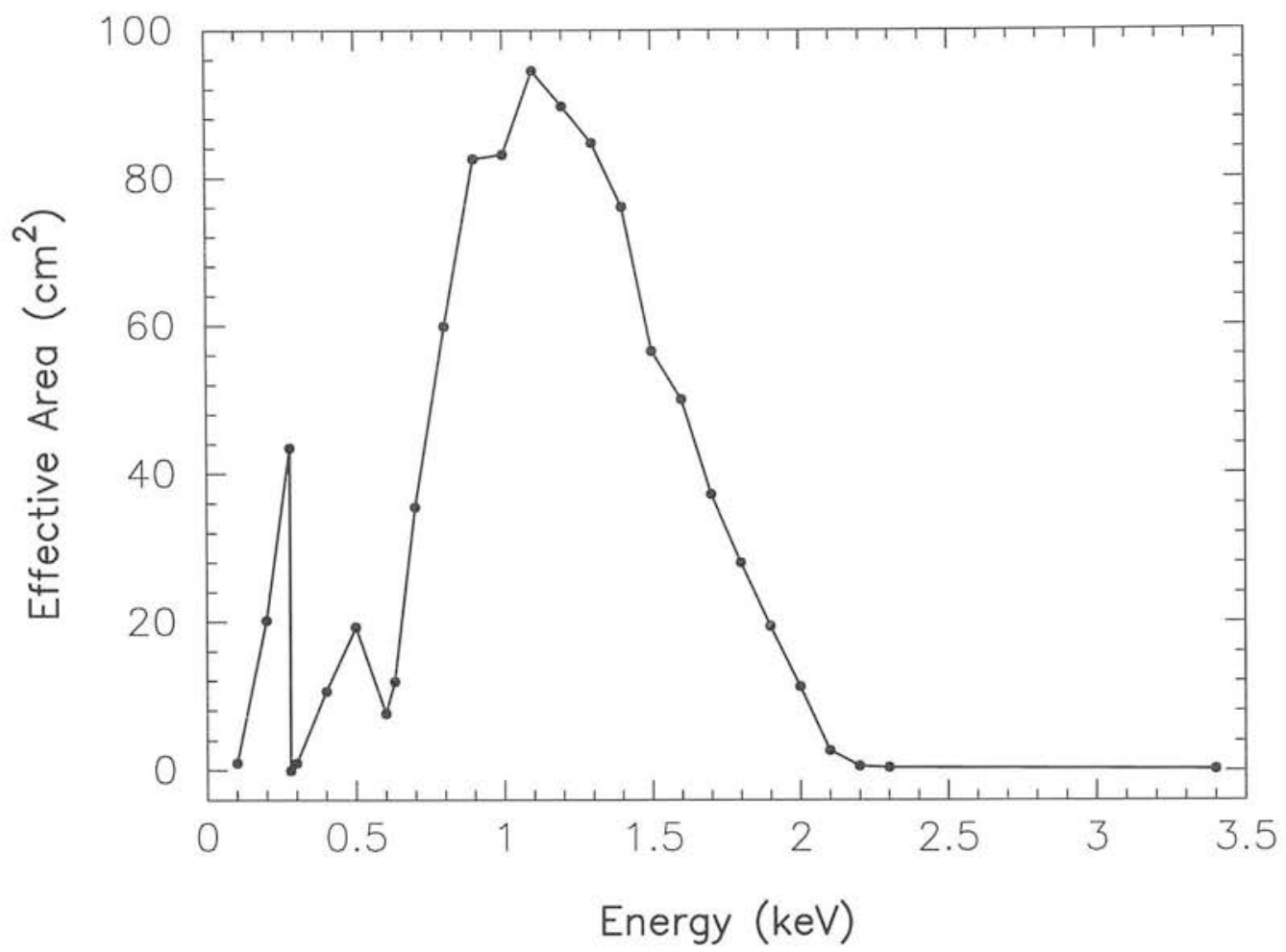

Figure 2.1: The on-axis effective area of the ROSAT HRI

with a thin layer of gold to enhance the X-ray reflectivity.

\subsubsection{The High Resolution Imager}

The ROSAT HRI was formed by two cascaded microchannel plates (MCPs) each one consisting of a large number of multiplier tubes assembled together. The detector had a total of $8192 \times 8192$ 'pixels' defined by a crossed grid position readout system. The HRI provided a $38 \times 38$ arc-min ${ }^{2}$ field of view and had very crude energy resolution. The plate scale was $0.499 \pm 0.001$ arc seconds per pixel.

The on-axis effective area of the XRT-HRI system was the product of the HRI quantum efficiency and the XRT collecting area and can be seen in figure 2.1. The XRT vignetting function at $1.0 \mathrm{keV}$ is well fitted by the following parametric representation (David et al. 1998):

$$
V(\theta)=1.00-1.49 \times 10^{-3} \times \theta-3.07 \times 10^{-4} \times \theta^{2}
$$

Vignetting is less than a $10 \%$ effect within $10^{\prime}$ from the centre of the detector at all energies of interest. 


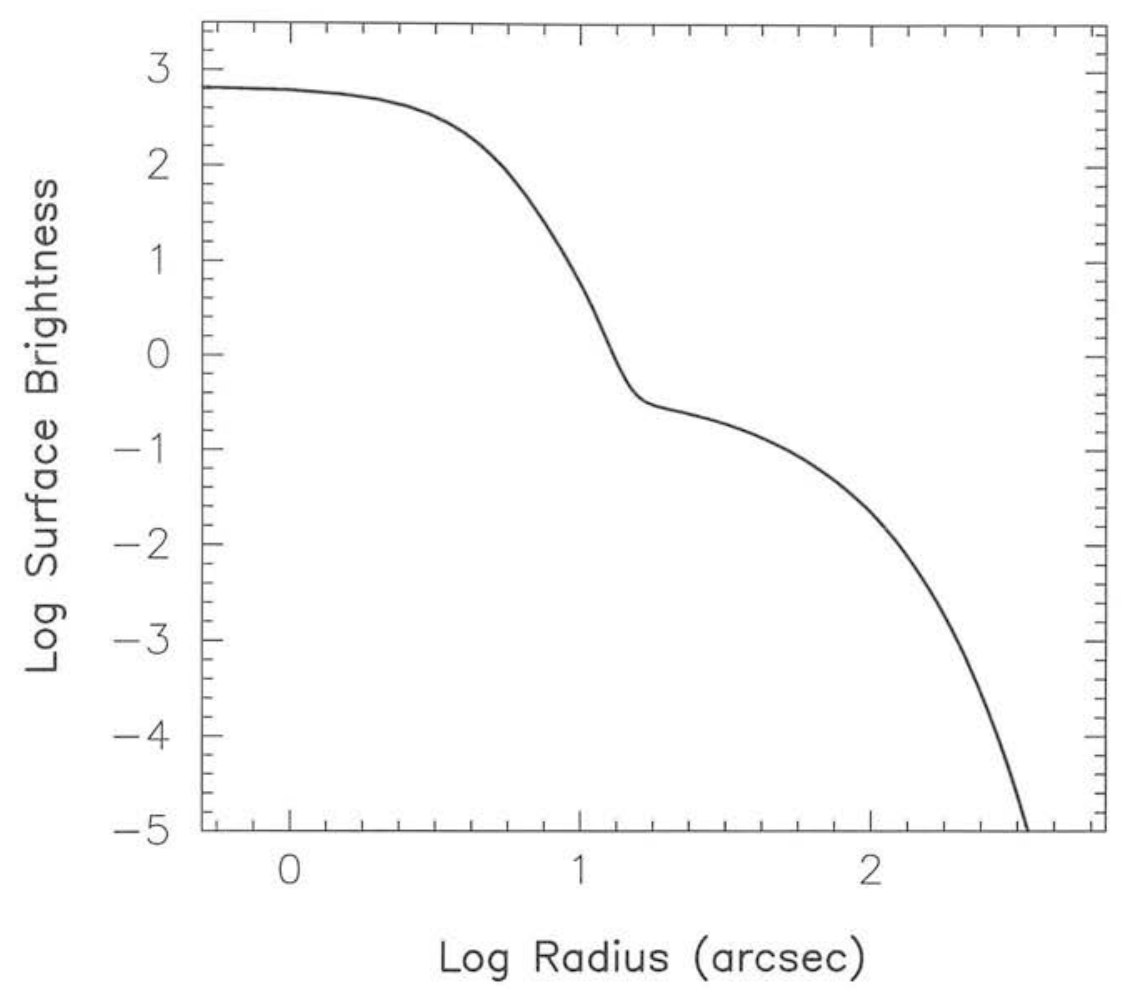

Figure 2.2: Parametric form of the HRI PSF

\section{The Point Spread Function}

The Point Spread Function (PSF) has three major components: the XRT PSF, the HRI PSF, and an extended halo caused by the presence of an electrostatic shield applied in front of the MCPs in order to lower the background emission. In some observations a variable ellipsoidal component, due to an imperfect aspect correction, can also be present (see below).

The in-flight, on-axis PSF has been determined from a long exposure of the X-ray source HZ 43. Figure 2.2 shows a parametric representation of the the azimuthally averaged PSF, given by the following expression (David et al. 1998):

$$
P S F(R)=A_{1} \exp ^{-\frac{1}{2}\left(\frac{R}{S_{1}}\right)^{2}}+A_{2} \exp ^{-\frac{1}{2}\left(\frac{R}{S_{2}}\right)^{2}}+A_{3} \exp ^{-\frac{1}{2}\left(\frac{R}{S_{3}}\right)}
$$

where $R$ is in arc seconds and

$$
\begin{array}{ll}
A_{1}=0.9638 & S_{1}=2.1858 \text { arc sec } \\
A_{2}=0.1798 & S_{2}=4.0419 \operatorname{arc~sec} \\
A_{3}=0.0009 & S_{3}=31.69 \operatorname{arc~sec}
\end{array}
$$




\begin{tabular}{|cc|}
\hline \hline $\mathrm{E}(\mathrm{keV})$ & \% photons \\
\hline 0.18 & 0.7 \\
0.28 & 1.3 \\
0.93 & 6.0 \\
1.49 & 11.0 \\
1.70 & 13.3 \\
\hline \hline
\end{tabular}

Table 2.1: Percentage of photons outside a $10^{\prime \prime}$ circle as a function of energy.

Scattering of photons on the mirror introduces an energy dependence to the PSF. The percentage of photons distributed outside a circle of radius $10^{\prime \prime}$ is shown in table 2.1 as a function of energy.

Changes in the PSF as a function of off-axis angle are dominated by the XRT PSF. A parametric form for the azimuthally averaged off-axis HRI PSF has been derived as an extension of the on-axis expression. The best fit values for the first gaussian are essentially independent of the off-axis angle. The width of the broader gaussian is parameterised by a cubic polynomial, given by (David et al. 1998):

$$
S_{2}=3.3+0.019 \times \theta-0.016 \times \theta^{2}+0.004 \times \theta^{3}
$$

where $\theta$ is the angle off-axis in arc minutes. Substituting this expression in equation 2.2 gives the general off-axis representation.

\section{Background}

The HRI in-orbit background is composed of several components:

- The internal background is believed to come mainly from residual radioactivity in the detector structure. This was measured on the ground with a rate of $\sim 1.5$ counts $\mathrm{s}^{-1}$ over the entire device and appears to be a stable component of the background, uniform over the detector.

- The X-ray background (XRB) contribution (comprised of both galactic and extragalactic components) varies across the sky, with a typical value in the ROSAT energy band of 1.0 count $\mathrm{s}^{-1}$ over the detector.

- The externally induced background from charged particles, earth scattered solar radiation, and auroral photons. The external background rates vary considerably 


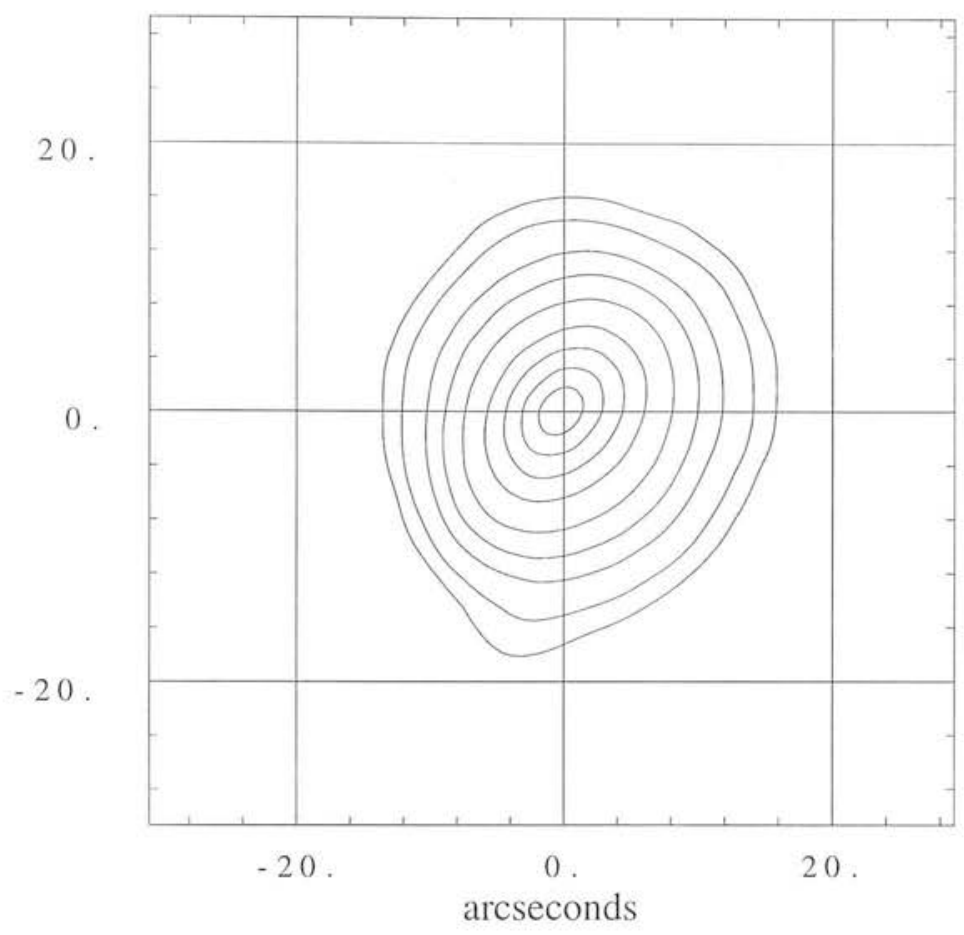

Figure 2.3: Contour map of HZ 43.

during an orbit and also from orbit to orbit, from a minimum of 1 count $\mathrm{s}^{-1}$ up to about 10 counts s ${ }^{-1}$.

\section{Absolute astrometry}

The comparison between measured and known positions of catalogued objects shows that the attitude solutions used to determine the source positions during XRT acquisitions include important errors, resulting in shifts of several arc-seconds (Briel et al. 1997). Compensation for such effects is possible whenever detected objects have previous X-ray observations or an unambiguous identification at other wavelengths.

\section{Problems with the aspect reconstruction}

During HRI pointing observations, ROSAT was wobbled by several arc-minutes to smooth out quantum efficiency variations across the detector (Morse 1994) with a period of $300-400$ seconds. The aspect solution to correct for this movement is mapped by following bright stars in the optical CCD star tracker.

Due to random errors in the aspect correction, the width of the core of the HRI images 

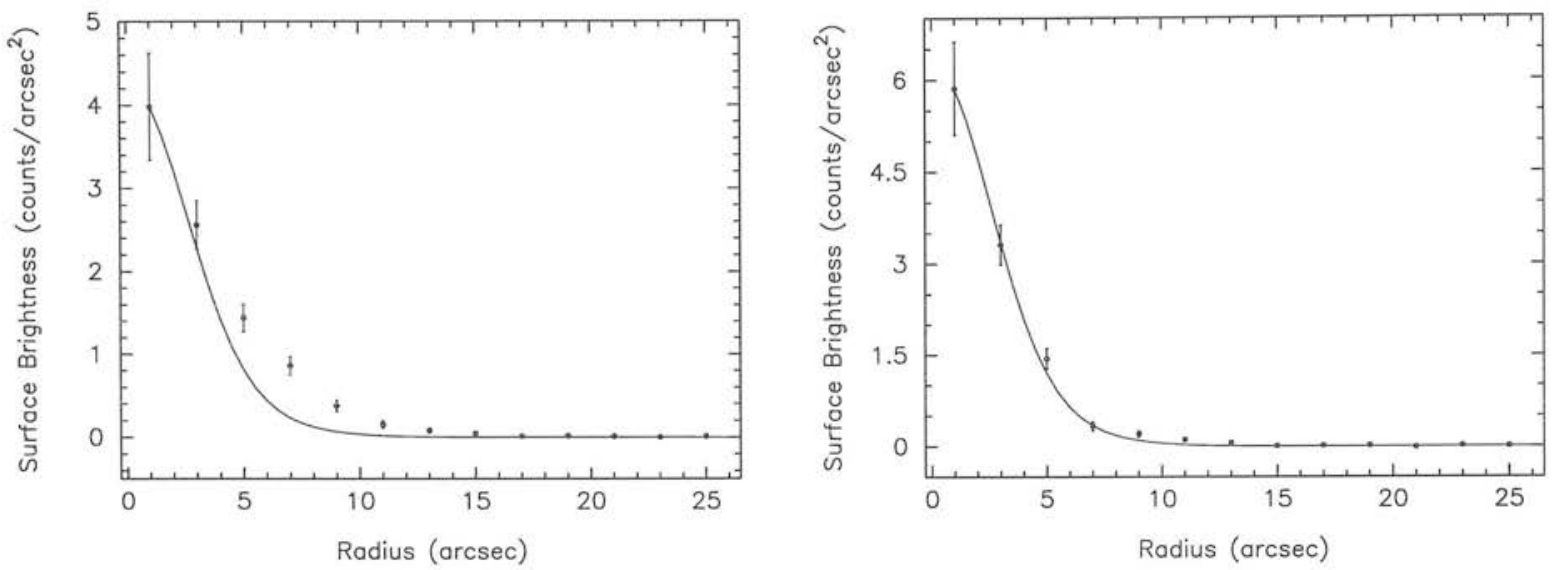

Figure 2.4: Observed and model PSF for two observations of a luminous X-ray source in NGC 5204 obtained 5 months apart.

varies. Fitting the functional form of the PSF (see expression 2.2) to data shows that the best fit values for $S_{1}$ and $S_{2}$ vary by $\lesssim 1^{\prime \prime}$ (David et al. 1998). The errors produce asymmetrical features in the PSF, normally seen as an ellipsoidal image. This effect can be seen in the contour map of the on-axis observation of HZ 43 shown in figure 2.3. The asymmetry is strongest between $5^{\prime \prime}$ and $10^{\prime \prime}$ from the centroid of the image and can have an amplitude of up to $30 \%$. These random variations of the PSF can hamper any efforts to identify possible extended emission around point sources.

To illustrate the variable nature of this elliptical component, figure 2.4 shows two observations of a luminous source in NGC 5204, acquired as part of this thesis. Both observations have similar exposure times and were obtained 5 months apart. While the first observation is well fitted by the model PSF (scaled to match the peak of the emission), a clear 'hump' appears during the second observation between $5^{\prime \prime}$ and $10^{\prime \prime}$ away from the central peak.

\subsection{Data reduction and analysis}

The data reported here come from pointed observations carried out with the ROSAT HRI. Table 1.1 shows those galaxies in the volume-limited sample with available data. Eighteen of the observations were awarded to the project through Announcement of Opportunity (AO) calls. For another 13 objects the data were retrieved from the public archives using ARnie (a World Wide Web interface to the databases and catalogues supported by the Leicester Database and Archive Service, LEDAS). Galaxies are identified in table 2.2 with an ' $\mathrm{N}$ ' (new data allocated to this project) or a 'P' (public data retrieved from the archive). All these observations were reduced and analysed as described below. 


\begin{tabular}{|c|c|c|c|c|c|}
\hline Galaxy & ROR Number & Observing Date & Livetime & Num OBIs & Origin \\
\hline NGC 147 & 400744 & $19 / 01 / 95$ & 14616.6 & 3 & $\mathrm{P}$ \\
\hline NGC 185 & 400743 & $19 / 01 / 95$ & 20881.3 & 2 & $\mathrm{P}$ \\
\hline NGC 205 & 600816 & $04 / 08 / 96$ & 27842.5 & 12 & $\mathrm{P}$ \\
\hline NGC 221 & 600600 & $19 / 07 / 94$ & 12533.5 & 6 & $\mathrm{P}$ \\
\hline \multirow[t]{2}{*}{ NGC 247} & 600622 & $29 / 06 / 94$ & 33701.1 & 6 & $\mathrm{P}$ \\
\hline & 600622 & $12 / 06 / 95$ & 17681.8 & 12 & - \\
\hline NGC 404 & 703894 & $04 / 01 / 97$ & 23562.6 & 2 & $\mathrm{~N}$ \\
\hline IC 342 & 600022 & $13 / 02 / 91$ & 18990.4 & 12 & $\mathrm{P}$ \\
\hline NGC 1560 & 702727 & $08 / 03 / 96$ & 17287.5 & 9 & $\mathrm{~N}$ \\
\hline NGC 2366 & 702732 & $31 / 03 / 96$ & 31465.5 & 7 & $\mathrm{~N}$ \\
\hline NGC 2403 & 600767 & $18 / 09 / 95$ & 26244.7 & 12 & $\mathrm{P}$ \\
\hline \multirow[t]{2}{*}{ NGC 2976} & 600759 & $04 / 10 / 94$ & 26238.1 & 12 & $\mathrm{P}$ \\
\hline & 600759 & $12 / 04 / 95$ & 23244.3 & 6 & - \\
\hline A $0951+68$ & 703895 & $29 / 03 / 97$ & 14240.2 & 3 & $\mathrm{~N}$ \\
\hline UGC 6456 & 703896 & $01 / 04 / 97$ & 13027.7 & 4 & $\mathrm{~N}$ \\
\hline NGC 3738 & 703897 & $01 / 11 / 97$ & 13711.2 & 9 & $\mathrm{~N}$ \\
\hline NGC 4136 & 702734 & $25 / 06 / 96$ & 1951.0 & 1 & $\mathrm{~N}$ \\
\hline NGC 4144 & 703898 & $11 / 11 / 97$ & 11083.4 & 8 & $\mathrm{~N}$ \\
\hline \multirow[t]{2}{*}{ NGC 4150} & 600762 & $25 / 12 / 94$ & 3730.1 & 2 & $\mathrm{~N}$ \\
\hline & 600762 & $23 / 05 / 95$ & 10353.8 & 5 & - \\
\hline \multirow[t]{2}{*}{ NGC 4236} & 600763 & $01 / 12 / 94$ & 7543.2 & 3 & $\mathrm{~N}$ \\
\hline & 600763 & $10 / 04 / 95$ & 3390.6 & 2 & - \\
\hline $\mathrm{NGC} 4244$ & 702724 & $20 / 06 / 96$ & 8630.9 & 4 & $\mathrm{~N}$ \\
\hline NGC 4395 & 702725 & $23 / 06 / 96$ & 11252.5 & 2 & $\mathrm{~N}$ \\
\hline NGC 4605 & 702729 & $16 / 06 / 96$ & 2158.4 & 1 & $\mathrm{~N}$ \\
\hline \multirow{2}{*}{ NGC 4736} & 600678 & $07 / 12 / 94$ & 111870.5 & 10 & $\mathrm{P}$ \\
\hline & 600769 & $25 / 12 / 94$ & 27033.8 & 4 & $\mathrm{P}$ \\
\hline \multirow[t]{3}{*}{ NGC 4826} & 600715 & $09 / 07 / 95$ & 10042.8 & 3 & $\mathrm{P}$ \\
\hline & 703900 & $12 / 06 / 97$ & 9248.9 & 3 & $\mathrm{~N}$ \\
\hline & 703900 & $08 / 01 / 98$ & 5342.2 & 4 & - \\
\hline \multirow[t]{2}{*}{ NGC 5204} & 702723 & $31 / 12 / 95$ & 14683.0 & 5 & $\mathrm{~N}$ \\
\hline & 702723 & $02 / 05 / 96$ & 13554.1 & 5 & - \\
\hline \multirow[t]{2}{*}{ NGC 5236} & 600024 & $20 / 01 / 93$ & 23316.5 & 7 & $\mathrm{P}$ \\
\hline & 600024 & $30 / 07 / 94$ & 23920.5 & 11 & - \\
\hline NGC 5238 & 702733 & $13 / 06 / 96$ & 23163.1 & 5 & $\mathrm{~N}$ \\
\hline \multirow[t]{2}{*}{ NGC 5457} & 600092 & $09 / 01 / 92$ & 18452.8 & 7 & $\mathrm{P}$ \\
\hline & 600383 & $10 / 12 / 92$ & 32361.1 & 4 & $\mathrm{P}$ \\
\hline NGC 6503 & 600618 & $08 / 03 / 94$ & 14640.6 & 2 & $\mathrm{~N}$ \\
\hline \multirow[t]{2}{*}{ NGC 6946} & 600501 & $14 / 05 / 94$ & 59885.3 & 33 & $\mathrm{P}$ \\
\hline & 600718 & $13 / 08 / 95$ & 21514.3 & 12 & $\mathrm{P}$ \\
\hline
\end{tabular}

Table 2.2: ROSAT HRI observations 


\subsubsection{Standard processing at MPE}

The data produced by ROSAT are processed at the ROSAT Science Data Centers (RSDC) located at Garching (MPE-Germany) and Greenbelt (US). Each pointed observation has an identification number consisting of 6 digits (the ROSAT Observation Request, ROR). The ROSAT Standard Analysis Software System (SASS) consists of a pipeline with four major steps:

- checking and saving of incoming data;

- handling of raw time-ordered telemetry data on a day-by-day basis;

- application of the aspect solution and screening of the data for bad observing periods (e.g., bad aspect solution, high background, etc);

- merging of observational intervals (OBIs) belonging to the same target, detection of sources, and determination of source properties.

The last step in the pipeline generates the scientific output for the data. During this step sources are detected using a 'sliding-box' detection algorithm and checked for possible time variability.

The data delivered by the RSDC to the observers consist of a series of Flexible Image Transport System (FITS) files, containing the photon events file, the source analysis results, orbit and aspect data, processing history, and postscript files with the most important results from the SASS analysis and results from a cross correlation of SIMBAD and detected HRI sources.

\section{2 .2 Observations}

For several galaxies more than one exposure was available. This could be because several observations were requested, or because a single request was scheduled as several observations months apart.

In table 2.2 observations of the same galaxy associated with different requests are distinguished by their ROR numbers. If a single request was scheduled in more than one observation only one ROR is given but the different observing dates distinguish between the individual observations. 
The livetime of each observation is also given in table 2.2. The livetime is equal to the cumulative number of seconds during which telemetered data met all the screening requirements mentioned above, corrected by the dead time of the detector (time when the detector is unable to record events because it is processing a prior event). For an event with a count rate of 100 counts $\mathrm{s}^{-1}$, the dead time losses will be about $4 \%-15 \%$ (ROSAT Call for Proposals 1991). For all the observations reported here the dead time corrections were found to be $\sim 1 \%$.

\subsubsection{Analysis procedure}

The aim of the analysis is to extract positions and fluxes or upper limits for all the $\mathrm{X}$ ray sources within a $\sim 6^{\prime} \times 6^{\prime}$ region centered on the position of the galaxy. In this way the X-ray field matches the size of the optical frames obtained using the Jacobus Kapteyn Telescope (JKT) (Johnson 1997). For each galaxy an isointensity contour map was overlaid onto the optical image. No attempt has been made to systematically study the temporal behaviour of the sources, although some interesting individual cases will be mentioned later (section 2.4).

The reduction and analysis of the ROSAT HRI data was done using PRos, an X-ray analysis software system designed to run under the Image Reduction and Analysis Facility (IRAF), distributed and supported by NOAO (National Optical Astronomy Observatories) and STARLINK.

The initial photon event files were converted into IRAF compatible QPOE (Quick Position Ordered Events) files. These are arrays of photons identified by several quantities, including the position on the detector, pulse height amplitude (PHA) and arrival time.

For each galaxy with more than one exposure, the different QPOE files were coadded, merging their respective livetimes. Using the sources in the field, the images were inspected and corrected for systematic shifts in the positions before co-adding. The positions were determined using a centroid algorithm. Whenever possible an optical identification was used to check the pointing of the individual observations.

\section{Source identification}

The next step was the identification of all point sources in the images. Tests carried out using a PROs routine to detect X-ray sources (DETECT) did not improve on the results 
given in the SASS report. Both algorithms are based on a 'sliding-box' routine: a square detect cell of size $d$ is scanned through the image at equally spaced steps $d / 3$ in both coordinates. At each location, the signal-to-noise ratio of a source assumed to be in the detect cell is calculated, based on an estimation of the background noise. Locations containing local peaks with signal-to-noise ratios above a threshold are identified and best estimate positions of the maximum are determined. A final estimation of the total counts and the background in a detect cell centered on each final position is calculated which depends on the assumed shape for the PSF. The routine is repeated for different cell sizes.

The main difference between the algorithms implemented by DETECT and SASS is in the background used. While DETECT uses a local background determined from a frame of width $d / 3$ immediately surrounding the detect cell, SASS uses a model background map. The background map is calculated from several components using ground based calibration data (e.g., the internal background) and in-flight observations (e.g., solar scattered emission).

During the tests DETECT was normally able to identify the point sources reported by SASS, but failed to identify some extended sources, since the local background was a bad estimate.

Using the SASS source list as a starting point, the following steps were followed to identify all source candidates in the X-ray images:

- the images were binned into $2^{\prime \prime}$ pixels and smoothed using Gaussians with $\sigma=2^{\prime \prime}, 4^{\prime \prime}$ and $8^{\prime \prime}$; the smoothed images were inspected visually to evaluate the existence of candidate sources not reported by SASS and to exclude obvious spurious sources;

- whenever more than one exposure was available for a target the different QPOE files were compared so that variable sources that might appear weak in the coadded image could be recognised in the individual frames where they might have been more luminous;

- optical images with X-ray isocontours were generated in order to look for weak sources with optical counterparts (see section 2.3).

For each source candidate a background subtracted count number was obtained from the coadded QPOE file. A circular aperture of radius 10" was adopted for all point sources which should encircle $\sim 99 \%$ of the photons at $0.2 \mathrm{keV}$ and $\sim 87 \%$ of the photons at 1.7 $\mathrm{keV}$ for nearly on-axis sources (see table 2.1 ). The pixel coordinates were obtained from 
the SASS report or by using a separate centroid algorithm. To estimate the background one or two large circular regions, free of evident X-ray sources and away from the galaxy, were used. These regions normally lay outside the $\sim 6^{\prime} \times 6^{\prime}$ central image.

A final list of sources for each galaxy was created with all sources that comply with at least one of the following criteria:

- have a signal-to-noise ratio above 2.5 in the coadded image;

- have a signal-to-noise ratio above 2.5 in at least one of the individual images;

- have a lower signal-to-noise ratio (between 1.5 and 2.5) and an optical counterpart.

For sources with an extended component an additional, larger aperture was used to estimate its contribution. The size of the aperture was determined from the radial profile of the source. For galaxies without nuclear X-ray source detections, upper limits were established using a $10^{\prime \prime}$ aperture located at the nucleus. They were computed as $2 \sigma$, where $\sigma$ is the measured noise in the background subtracted aperture.

To find the count rates the total livetime of the coadded images was used (see table 2.2).

\section{$\mathrm{X}$-ray fluxes and luminosities}

The conversion of the HRI count rates to fluxes was done assuming a Bremsstrahlung spectrum with $k T=5 \mathrm{keV}$, an energy range $0.1-2.4 \mathrm{keV}$, and a Galactic line of sight absorption derived from the $21 \mathrm{~cm}$ line of atomic hydrogen (Stark et al. 1992), listed in table 2.3. This choice of parameters is justified by typical galactic X-ray spectral properties (Kim, Fabbiano \& Trinchieri 1992). Fluxes were converted to luminosities assuming the distances in table 1.1. Fluxes and luminosities inferred from a Bremsstrahlung spectrum with $k T=1.0$ or $0.1 \mathrm{keV}$, or a power law model with index $\alpha=1.0$ or 1.5 , can be calculated using the conversion factors in table 2.3 for each galaxy.

\section{Contour maps}

Isointensity contour maps were produced for all the coadded images. All the QPOE files were binned into $2^{\prime \prime} \times 2^{\prime \prime}$ pixels and then smoothed using a Gaussian with $\sigma=4^{\prime \prime}$. The choice of binning and Gaussian sizes was based on the best possible representation of 


\begin{tabular}{|c|c|c|c|c|c|}
\hline \multirow[t]{2}{*}{ Galaxy } & \multirow{2}{*}{$\begin{array}{l}\log N_{H} \\
\left(\mathrm{~cm}^{-2}\right)\end{array}$} & \multicolumn{2}{|c|}{ Bremsstrahlung } & \multicolumn{2}{|c|}{ Power Law } \\
\hline & & $k T=1.0 \mathrm{keV}$ & $k T=0.1 \mathrm{keV}$ & $\alpha=1.0$ & $\alpha=1.5$ \\
\hline NGC 147 & 21.02 & 1.21 & 28.36 & 2.26 & 1.42 \\
\hline NGC 185 & 21.04 & 1.22 & 28.70 & 2.27 & 1.42 \\
\hline NGC 205 & 20.82 & 1.17 & 11.10 & 2.07 & 1.37 \\
\hline $\mathrm{NGC} 221$ & 20.81 & 1.17 & 10.78 & 2.07 & 1.37 \\
\hline $\mathrm{NGC} 247$ & 20.17 & 1.01 & 1.96 & 1.36 & 1.14 \\
\hline NGC 404 & 20.70 & 1.15 & 8.28 & 1.98 & 1.34 \\
\hline IC 342 & 21.48 & 1.30 & 49.67 & 2.48 & 1.48 \\
\hline NGC 1560 & 21.06 & 1.22 & 29.06 & 2.27 & 1.43 \\
\hline NGC 2366 & 20.59 & 1.11 & 4.62 & 1.78 & 1.29 \\
\hline NGC 2403 & 20.62 & 1.12 & 5.23 & 1.83 & 1.30 \\
\hline NGC 2976 & 20.65 & 1.13 & 6.05 & 1.89 & 1.32 \\
\hline A $0951+68$ & 20.63 & 1.12 & 5.48 & 1.85 & 1.31 \\
\hline UGC 6456 & 20.56 & 1.10 & 4.15 & 1.74 & 1.27 \\
\hline NGC 3738 & 20.01 & 0.99 & 1.67 & 1.27 & 1.10 \\
\hline NGC 4136 & 20.20 & 1.02 & 2.03 & 1.38 & 1.15 \\
\hline NGC 4144 & 20.16 & 1.01 & 1.94 & 1.36 & 1.14 \\
\hline NGC 4150 & 20.19 & 1.02 & 2.01 & 1.38 & 1.15 \\
\hline NGC 4236 & 20.26 & 1.03 & 2.20 & 1.43 & 1.16 \\
\hline NGC 4244 & 20.27 & 1.03 & 2.23 & 1.43 & 1.17 \\
\hline NGC 4395 & 20.12 & 1.00 & 1.85 & 1.33 & 1.13 \\
\hline NGC 4605 & 20.26 & 1.03 & 2.20 & 1.43 & 1.16 \\
\hline NGC 4736 & 20.15 & 1.01 & 1.92 & 1.35 & 1.13 \\
\hline NGC 4826 & 20.42 & 1.06 & 2.88 & 1.57 & 1.22 \\
\hline NGC 5204 & 20.18 & 1.01 & 1.98 & 1.37 & 1.14 \\
\hline NGC 5236 & 20.63 & 1.12 & 5.48 & 1.8 .5 & 1.31 \\
\hline NGC 5238 & 20.04 & 0.99 & 1.71 & 1.29 & 1.11 \\
\hline NGC 5457 & 20.07 & 1.00 & 1.76 & 1.30 & 1.11 \\
\hline NGC 6503 & 20.61 & 1.12 & 5.01 & 1.82 & 1.30 \\
\hline NGC 6946 & 21.31 & 1.26 & 36.43 & 2.37 & 1.45 \\
\hline
\end{tabular}

Table 2.3: Factors to convert fluxes obtained assuming Bremsstrahlung emission with $k T=5.0$ to Bremsstrahlung emission with $k T=1.0$ or $0.1 \mathrm{keV}$, or a power law model with index $\alpha=1.0$ or 1.5 . 
the presence of existing sources and their strength. Contours were drawn at $2.5^{n}$ times the standard deviation per (binned smoothed) pixel in the smoothed background, where $n=1,2,3$, and so on. With this selection of contour intensities bright sources do not present contour crowding. For each map, the background fluctuation was calculated from the same region used earlier (when measuring source fluxes in the raw data).

\subsection{The X-ray Atlas}

The results obtained for those observations listed in table 2.2 after following the reduction and analysis procedures detailed in the previous section will be given here.

The atlas consists of maps of isointensity contour levels overlaid on optical images. The size of the images is $\sim 6^{\prime} \times 6^{\prime}$. Whenever a JKT image was not available (see table 1.1 ), a $6^{\prime} \times 6^{\prime}$ Digital Sky Survey plate was used. For each map the exposure times of the ROSAT observations are given in the figure captions. The atlas is ordered by increasing values of right ascension.

All detected X-ray point sources fluxes are given in table 2.4. The sources are ordered by increasing values of right ascension. For each source in table 2.4 successive columns list the measured signal to noise ratio, the flux corrected for Galactic absorption in the 0.1-2.4 keV band, and the luminosity found assuming the distances shown in table 1.1. Comments on particular sources are given in the last column (nuclear sources are also noted in this way). When there is evidence of an extended nuclear component, the source is listed twice: the flux measured in a $10^{\prime \prime}$ radius aperture is followed by the total flux observed in a larger aperture (the radius of the second aperture is given in the comments).

Upper limits $(2 \sigma)$ were obtained for galaxies without detected nuclear sources and can be found in table 2.5 .

In addition to the galaxies listed in table 2.2 the results for four galaxies with comprehensive studies of their X-ray emission from HRI observations have been obtained directly from the literature. Luminosities for the detected point sources (within $\leq 3^{\prime}$ of the nucleus) are shown in table 2.6. These values will be used during the analysis in chapter 5. 


\begin{tabular}{|c|c|c|c|c|c|}
\hline Galaxy & Source & $\mathrm{S} / \mathrm{N}$ & $\begin{array}{l}\text { Corrected Flux } \\
\left(\mathrm{ergs} \mathrm{s}^{-1} \mathrm{~cm}^{-2}\right)\end{array}$ & $\begin{array}{l}\text { Luminosity } \\
\left(\mathrm{ergs} \mathrm{s}^{-1}\right)\end{array}$ & Comments \\
\hline NGC 205 & $\mathrm{X} 1$ & 3.82 & $7.93 \times 10^{-14}$ & $4.01 \times 10^{36}$ & Probably not associated with NGC 205 \\
\hline $\mathrm{NGC} 221$ & X1 & 10.60 & $6.37 \times 10^{-13}$ & $3.22 \times 10^{37}$ & \\
\hline NGC 247 & $\begin{array}{l}X 1 \\
X 2\end{array}$ & $\begin{array}{c}22.39 \\
5.99\end{array}$ & $\begin{array}{l}7.37 \times 10^{-13} \\
9.15 \times 10^{-14}\end{array}$ & $\begin{array}{l}1.20 \times 10^{39} \\
1.49 \times 10^{38}\end{array}$ & \\
\hline NGC 404 & $\mathrm{X} 1$ & 3.34 & $7.48 \times 10^{-14}$ & $1.69 \times 10^{38}$ & Nuclear \\
\hline IC 342 & $\begin{array}{l}\mathrm{X} 1 \\
\mathrm{X} 2 \\
\mathrm{X} 3\end{array}$ & $\begin{array}{l}2.31 \\
9.27 \\
6.48\end{array}$ & $\begin{array}{l}9.58 \times 10^{-14} \\
6.07 \times 10^{-13} \\
3.44 \times 10^{-13}\end{array}$ & $\begin{array}{l}4.24 \times 10^{38} \\
2.68 \times 10^{39} \\
1.52 \times 10^{39}\end{array}$ & $\begin{array}{l}\text { Re-analysis of the observations reported } \\
\text { by Bregman, Cox \& Tomisaka (1993)-BCT } \\
\text { Low S/N (see text); source } 6 \text { in BCT } \\
\text { Nuclear; marginally extended; source } 8 \text { in BCT } \\
\text { Possible optical counterpart; source } 9 \text { in BCT }\end{array}$ \\
\hline NGC 2403 & $\begin{array}{l}\mathrm{X} 1 \\
\mathrm{X} 2 \\
\mathrm{X} 3 \\
\mathrm{X} 4\end{array}$ & $\begin{array}{l}15.19 \\
2.92 \\
7.88 \\
2.08\end{array}$ & $\begin{array}{l}5.56 \times 10^{-13} \\
5.67 \times 10^{-14} \\
1.89 \times 10^{-13} \\
4.29 \times 10^{-14}\end{array}$ & $\begin{array}{l}3.01 \times 10^{39} \\
3.07 \times 10^{38} \\
1.02 \times 10^{39} \\
2.33 \times 10^{38}\end{array}$ & $\begin{array}{l}\text { Possible very faint optical counterpart } \\
\text { Low S/N; giant HII region (Drissen \& Roy 1996) }\end{array}$ \\
\hline NGC 2976 & $\mathrm{X} 1$ & 8.75 & $1.32 \times 10^{-13}$ & $2.15 \times 10^{38}$ & \\
\hline A $0951+68$ & $\mathrm{X} 1$ & 4.79 & $1.06 \times 10^{-13}$ & $4.00 \times 10^{37}$ & Probably not associated with A $0951+68$ \\
\hline UGC 6456 & X1 & 13.85 & $8.82 \times 10^{-13}$ & $6.03 \times 10^{38}$ & \\
\hline NGC 3738 & $\begin{array}{l}\mathrm{X} 1 \\
\mathrm{X} 2\end{array}$ & $\begin{array}{l}4.74 \\
2.46\end{array}$ & $\begin{array}{l}1.18 \times 10^{-13} \\
5.25 \times 10^{-14}\end{array}$ & $\begin{array}{l}5.22 \times 10^{38} \\
2.32 \times 10^{38}\end{array}$ & $\begin{array}{l}\text { Probably not associated with NGC } 3738 \\
\text { Probably not associated with NGC } 3738\end{array}$ \\
\hline NGC 4136 & $\mathrm{X} 1$ & 2.15 & $2.19 \times 10^{-13}$ & $1.26 \times 10^{39}$ & Diffuse blue optical counterpart \\
\hline NGC 4144 & X1 & 3.09 & $8.77 \times 10^{-14}$ & $3.60 \times 10^{38}$ & \\
\hline NGC 4150 & $\mathrm{X} 1$ & 15.84 & $1.02 \times 10^{-12}$ & $1.88 \times 10^{45}$ & $\begin{array}{l}\text { Background quasar }(z=0.52) \text {; see chapter } 3 \text {; } \\
\text { flux obtained assuming a power law spectrum } \\
\text { with } \alpha=1.0\end{array}$ \\
\hline NGC 4236 & $\mathrm{X} 1$ & 2.61 & $7.24 \times 10^{-14}$ & $9.21 \times 10^{37}$ & \\
\hline NGC 4395 & $\mathrm{X} 1$ & 1.67 & $3.73 \times 10^{-14}$ & $1.21 \times 10^{38}$ & $\begin{array}{l}\text { Nuclear; low } \mathrm{S} / \mathrm{N} \text {; flux obtained assuming a } \\
\text { power law spectrum with } \alpha=1.0 \text {; see chapter } 4\end{array}$ \\
\hline $\mathrm{NGC} 4736$ & $\begin{array}{l}\mathrm{X} 2 \\
\mathrm{X} 1 \\
\mathrm{X} 2 \\
\mathrm{X} 3 \\
\mathrm{X} 3\end{array}$ & $\begin{array}{l}11.85 \\
5.78 \\
18.78 \\
65.32 \\
84.49\end{array}$ & $\begin{array}{l}6.46 \times 10^{-13} \\
3.40 \times 10^{-14} \\
1.46 \times 10^{-13} \\
1.38 \times 10^{-12} \\
4.70 \times 10^{-12}\end{array}$ & $\begin{array}{l}2.10 \times 10^{39} \\
1.51 \times 10^{38} \\
6.46 \times 10^{38} \\
6.11 \times 10^{39} \\
2.08 \times 10^{40}\end{array}$ & $\begin{array}{l}\text { Nuclear } \\
\text { Total nuclear emission }\left(\mathrm{r}=100^{\prime \prime}\right)\end{array}$ \\
\hline
\end{tabular}

Table 2.4: ROSAT HRI fluxes and luminosities. Continued over. 


\begin{tabular}{|c|c|c|c|c|c|}
\hline Galaxy & Source & $\mathrm{S} / \mathrm{N}$ & $\begin{array}{l}\text { Corrected Flux } \\
\left(\mathrm{ergs} \mathrm{s}^{-1} \mathrm{~cm}^{-2}\right)\end{array}$ & $\begin{array}{l}\text { Luminosity } \\
\left(\mathrm{ergs} \mathrm{s}^{-1}\right)\end{array}$ & Comments \\
\hline \multicolumn{6}{|c|}{ NGC 4826} \\
\hline & $\mathrm{X} 1$ & 9.58 & $2.41 \times 10^{-13}$ & $9.19 \times 10^{38}$ & Nuclear \\
\hline & $\mathrm{X} 1$ & 13.03 & $7.73 \times 10^{-13}$ & $2.94 \times 10^{39}$ & Total nuclear emission $\left(\mathrm{r}=40^{\prime \prime}\right)$ \\
\hline & $\mathrm{X} 2$ & 2.27 & $3.98 \times 10^{-14}$ & $1.52 \times 10^{38}$ & Probably not associated with NGC 4826 \\
\hline \multicolumn{6}{|c|}{ NGC 5204} \\
\hline & $\mathrm{X} 1$ & 25.23 & $1.08 \times 10^{-12}$ & $5.86 \times 10^{39}$ & Faint optical counterpart \\
\hline \multicolumn{6}{|c|}{ NGC 5236} \\
\hline & $\mathrm{X} 1$ & 4.46 & $5.83 \times 10^{-14}$ & $2.06 \times 10^{38}$ & Variable \\
\hline & $\mathrm{X} 2$ & 6.07 & $8.23 \times 10^{-14}$ & $2.90 \times 10^{38}$ & \\
\hline & $\mathrm{X} 3$ & 26.69 & $8.75 \times 10^{-13}$ & $3.09 \times 10^{39}$ & Nuclear \\
\hline & X3 & 38.48 & $3.01 \times 10^{-12}$ & $1.06 \times 10^{40}$ & Total nuclear emission $\left(\mathrm{r}=100^{\prime \prime}\right)$ \\
\hline & $\mathrm{X} 4$ & 5.09 & $6.69 \times 10^{-14}$ & $2.36 \times 10^{38}$ & \\
\hline & $\mathrm{X} 5$ & 3.78 & $4.95 \times 10^{-14}$ & $1.75 \times 10^{38}$ & \\
\hline & $\mathrm{X} 6$ & 8.60 & $1.31 \times 10^{-13}$ & $4.61 \times 10^{38}$ & \\
\hline & $\mathrm{X} 7$ & 5.01 & $8.45 \times 10^{-14}$ & $2.98 \times 10^{38}$ & Variable \\
\hline & X8 & 4.13 & $5.38 \times 10^{-14}$ & $1.90 \times 10^{38}$ & Variable \\
\hline \multicolumn{6}{|c|}{ NGC 5457} \\
\hline & $\mathrm{X} 1$ & 4.13 & $3.88 \times 10^{-14}$ & $2.68 \times 10^{38}$ & Nuclear \\
\hline & $\mathrm{X} 2$ & 3.31 & $3.16 \times 10^{-14}$ & $2.19 \times 10^{38}$ & \\
\hline & $\mathrm{X} 3$ & 4.80 & $4.56 \times 10^{-14}$ & $3.15 \times 10^{38}$ & \\
\hline & $\mathrm{X} 4$ & 3.52 & $3.32 \times 10^{-14}$ & $2.30 \times 10^{38}$ & \\
\hline \multicolumn{6}{|c|}{ NGC 6503} \\
\hline & $\mathrm{X} 1$ & 3.01 & $7.86 \times 10^{-14}$ & $4.53 \times 10^{38}$ & \\
\hline & $\mathrm{X} 2$ & 2.51 & $6.47 \times 10^{-14}$ & $3.73 \times 10^{38}$ & Probably nuclear \\
\hline & $\mathrm{X} 2$ & 3.47 & $2.69 \times 10^{-13}$ & $1.55 \times 10^{39}$ & Total nuclear emission $\left(\mathrm{r}=30^{\prime \prime}\right)$ \\
\hline \multicolumn{6}{|c|}{ NGC 6946} \\
\hline & $\mathrm{X} 1$ & 11.85 & $2.05 \times 10^{-13}$ & $1.34 \times 10^{39}$ & \\
\hline & $\mathrm{X} 2$ & 2.67 & $4.19 \times 10^{-14}$ & $2.73 \times 10^{38}$ & \\
\hline & $\mathrm{X} 3$ & 10.73 & $1.77 \times 10^{-13}$ & $1.16 \times 10^{39}$ & Nuclear \\
\hline & $\mathrm{X} 3$ & 12.80 & $4.49 \times 10^{-13}$ & $2.92 \times 10^{39}$ & Total nuclear emission $\left(\mathrm{r}=50^{\prime \prime}\right)$ \\
\hline & $\mathrm{X} 4$ & 4.37 & $6.10 \times 10^{-14}$ & $3.98 \times 10^{38}$ & \\
\hline & $\mathrm{X} 5$ & 3.06 & $4.59 \times 10^{-14}$ & $2.99 \times 10^{38}$ & \\
\hline & $\mathrm{X} 6$ & 5.48 & $7.62 \times 10^{-14}$ & $4.97 \times 10^{38}$ & \\
\hline & $\times 7$ & 13.25 & $2.45 \times 10^{-13}$ & $1.59 \times 10^{39}$ & \\
\hline & $\mathrm{X} 8$ & 32.22 & $1.15 \times 10^{-12}$ & $7.53 \times 10^{39}$ & $\begin{array}{l}\text { Known SNR (see text); faint red optical } \\
\text { counterpart. }\end{array}$ \\
\hline & $\mathrm{X} 9$ & 5.13 & $7.11 \times 10^{-14}$ & $4.64 \times 10^{38}$ & \\
\hline
\end{tabular}

Table 2.4: ROSAT HRI fluxes and luminosities. 


\begin{tabular}{|l|c|c|}
\hline \hline Galaxy & $\begin{array}{c}\text { Corrected Upper Limit } \\
\left(\mathrm{ergs} \mathrm{s}^{-1} \mathrm{~cm}^{-2}\right)\end{array}$ & $\begin{array}{c}\text { Log luminosity } \\
\left(\mathrm{ergs} \mathrm{s}^{-1}\right)\end{array}$ \\
\hline NGC 147 & $5.53 \times 10^{-14}$ & 36.45 \\
NGC 185 & $4.58 \times 10^{-14}$ & 36.37 \\
NGC 205 & $4.21 \times 10^{-14}$ & 36.33 \\
NGC 221 & $5.32 \times 10^{-14}$ & 36.43 \\
NGC 247 & $1.76 \times 10^{-14}$ & 37.46 \\
NGC 1560 & $5.63 \times 10^{-14}$ & 38.10 \\
NGC 2366 & $2.82 \times 10^{-14}$ & 38.13 \\
NGC 2403 & $3.62 \times 10^{-14}$ & 38.29 \\
NGC 2976 & $2.60 \times 10^{-14}$ & 37.63 \\
A 0951+68 & $3.59 \times 10^{-14}$ & 37.00 \\
UGC 6456 & $5.15 \times 10^{-14}$ & 37.55 \\
NGC 3738 & $4.16 \times 10^{-14}$ & 38.27 \\
NGC 4136 & $1.92 \times 10^{-13}$ & 39.05 \\
NGC 4144 & $5.17 \times 10^{-14}$ & 38.33 \\
NGC 4150 & $6.35 \times 10^{-14}$ & 37.96 \\
NGC 4236 & $5.71 \times 10^{-14}$ & 37.86 \\
NGC 4244 & $7.26 \times 10^{-14}$ & 38.22 \\
NGC 4605 & $2.43 \times 10^{-13}$ & 38.97 \\
NGC 5204 & $2.96 \times 10^{-14}$ & 38.21 \\
NGC 5238 & $2.92 \times 10^{-14}$ & 38.20 \\
\hline \hline
\end{tabular}

Table 2.5: ROSAT HRI nuclear upper limits 


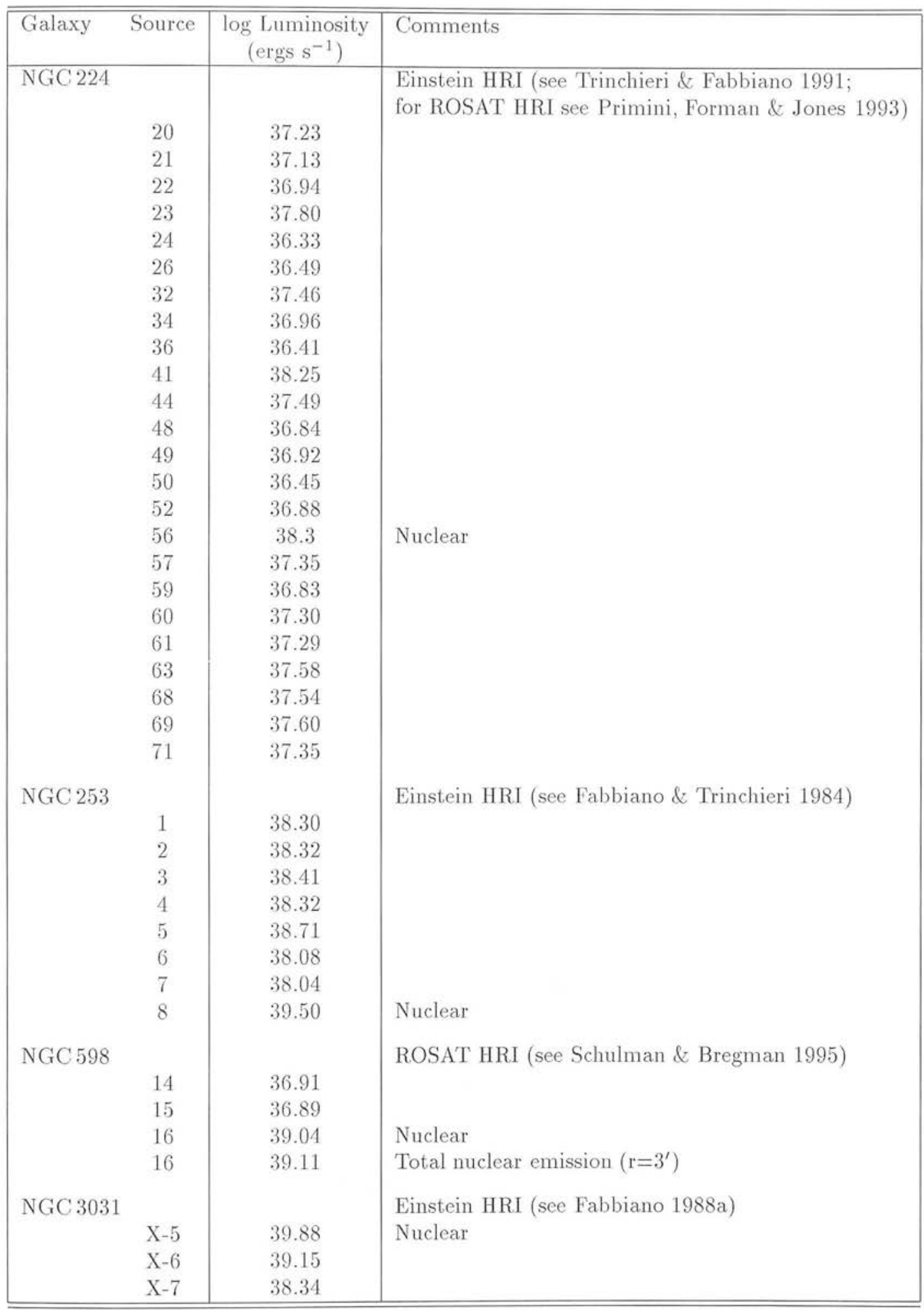

Table 2.6: HRI sources and their luminosities obtained directly from the literature for four well studied galaxies. Einstein luminosities were measured in the $0.2-4.0 \mathrm{keV}$ band-pass. These values will be used during the analysis in chapter 5 where a correction factor of 1.4 will be applied to account for the differences between the band-passes and sensitivities of ROSAT and Einstein (see section 5.4). 


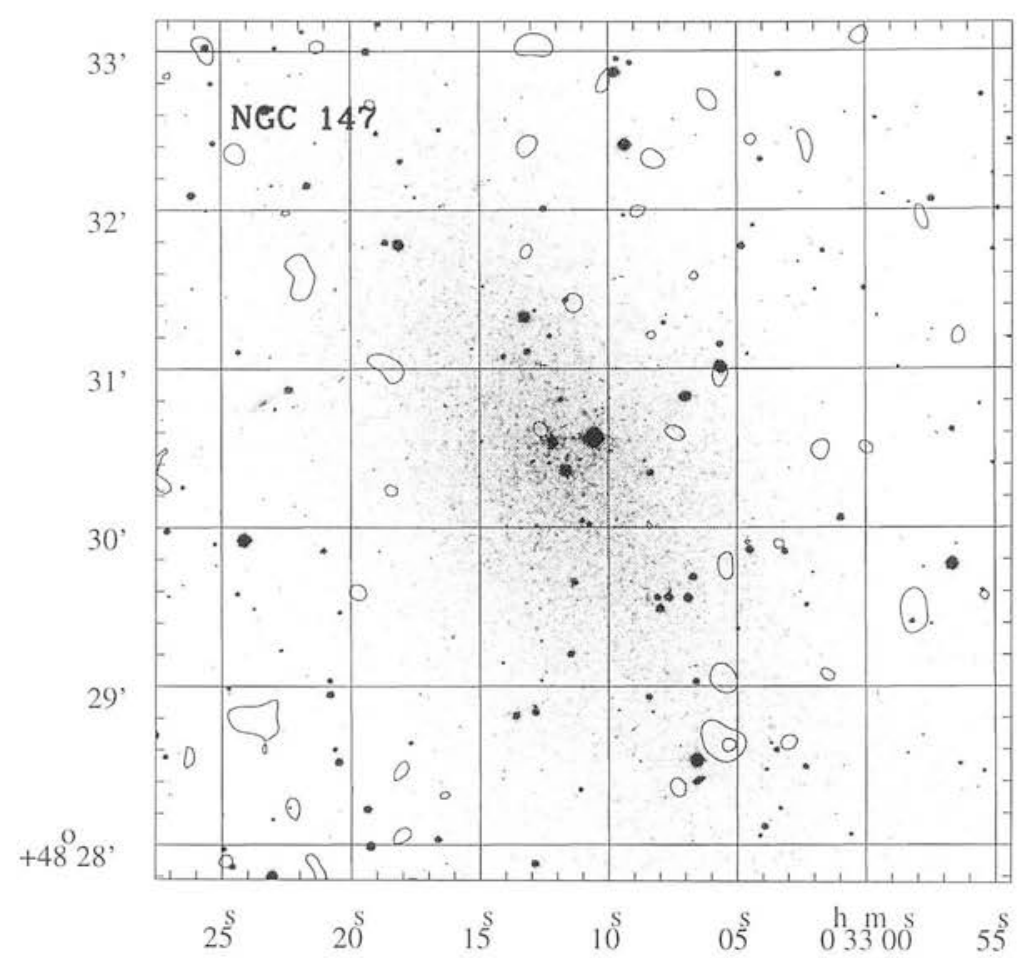

Figure 2.5: NGC147 X-ray contours overlaid on optical JKT image. ROSAT exposure time $=14616.6$ seconds.

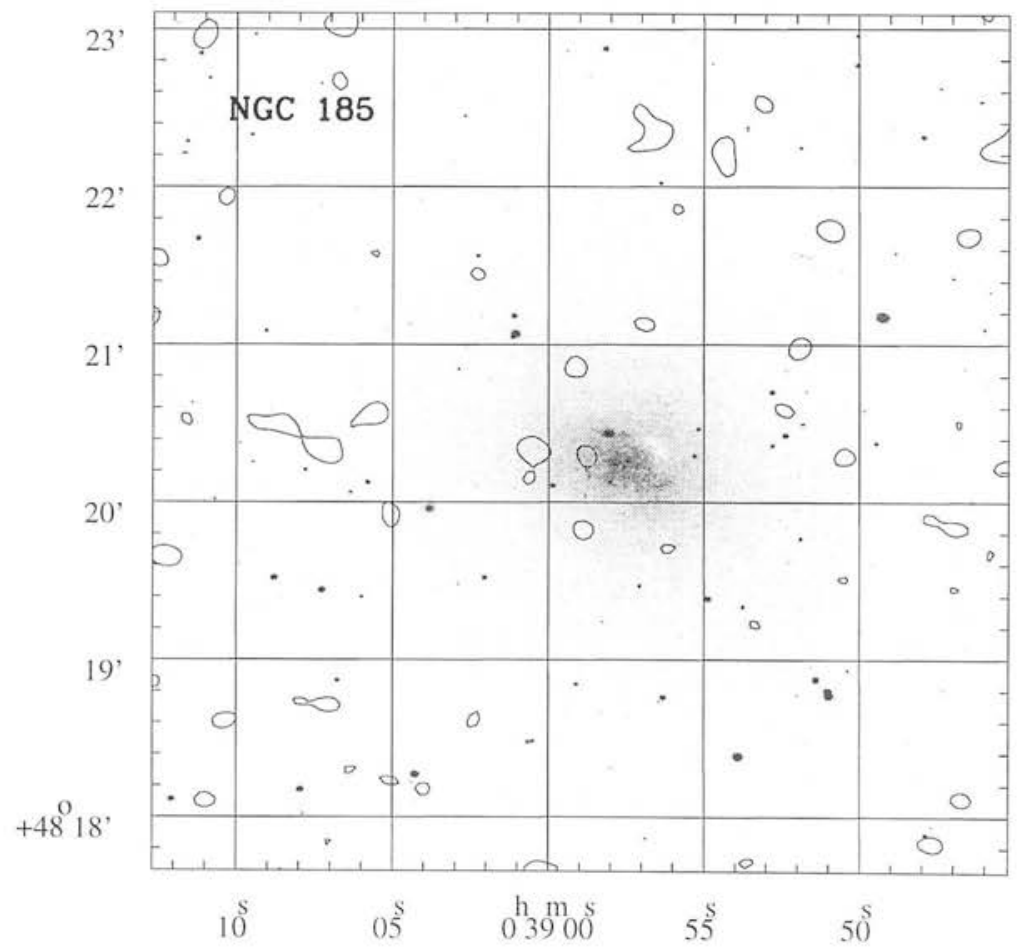

Figure 2.6: NGC $185 \mathrm{X}$-ray contours overlaid on optical JKT image. ROSAT exposure time $=20881.3$ seconds. 


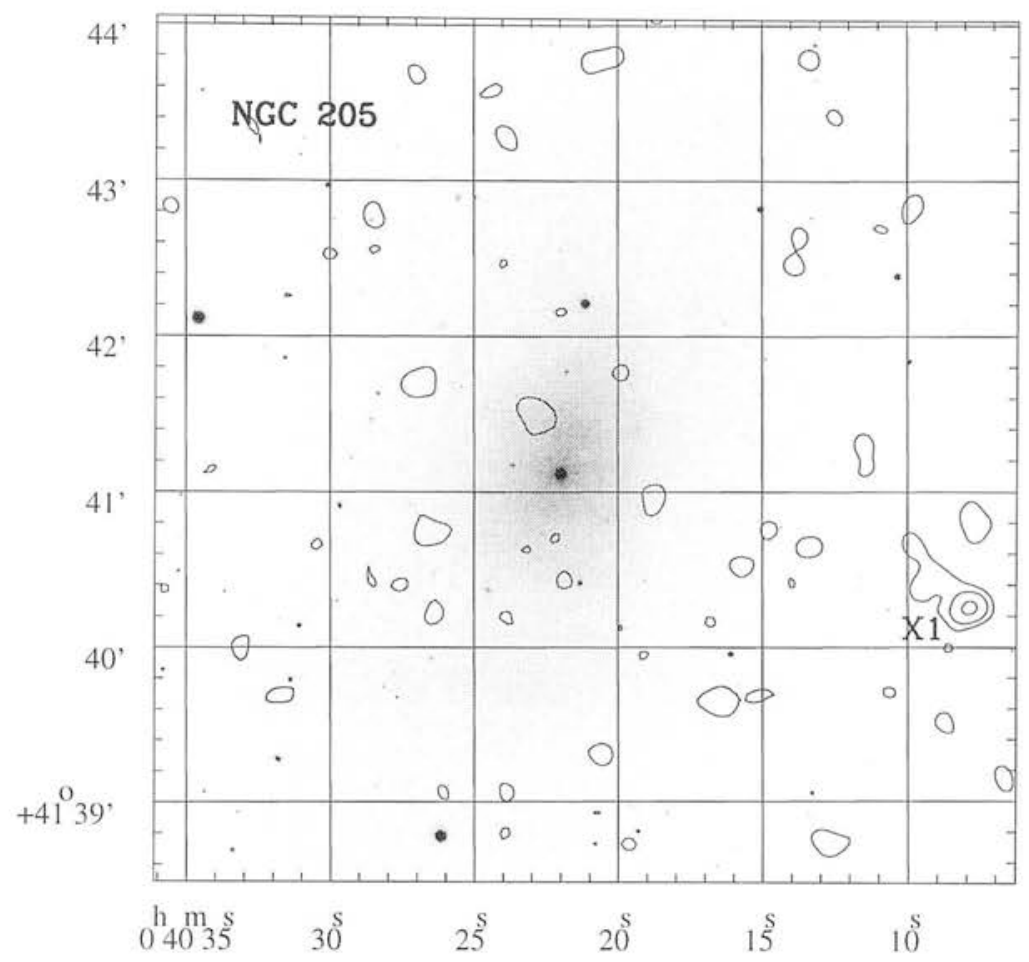

Figure 2.7: NGC 205 X-ray contours overlaid on optical JKT image. ROSAT exposure time $=27842.5$ seconds .

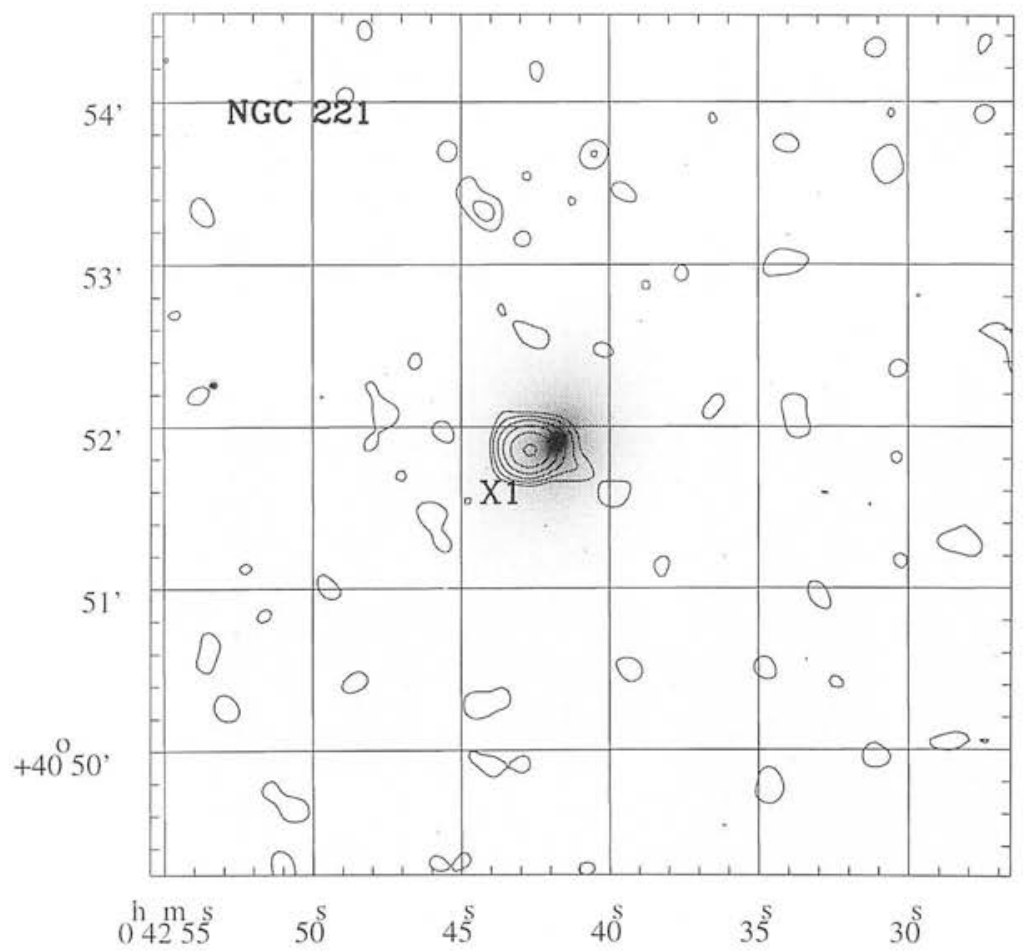

Figure 2.8: NGC221 X-ray contours overlaid on optical JKT image. ROSAT exposure time $=12533.5$ seconds. 


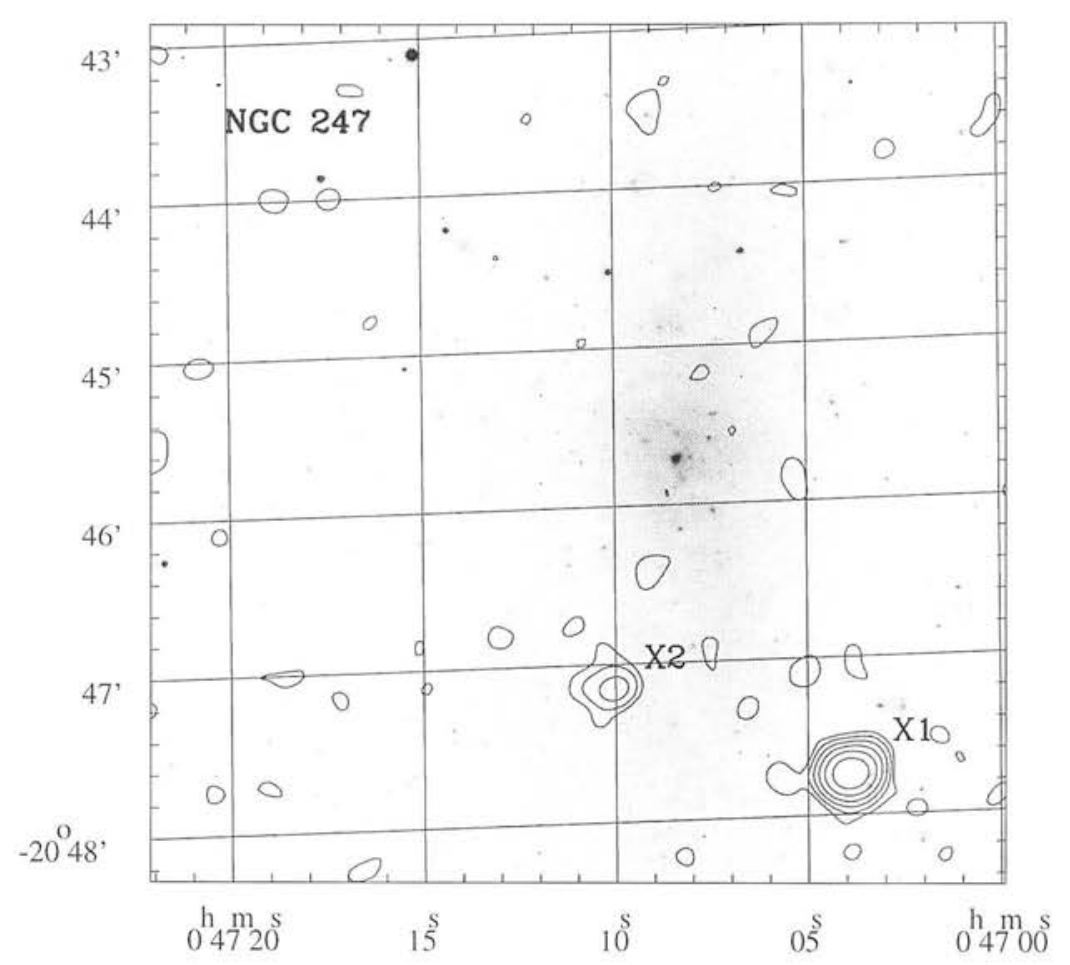

Figure 2.9: NGC 247 X-ray contours overlaid on optical JKT image. ROSAT exposure time $=51382.9$ seconds.

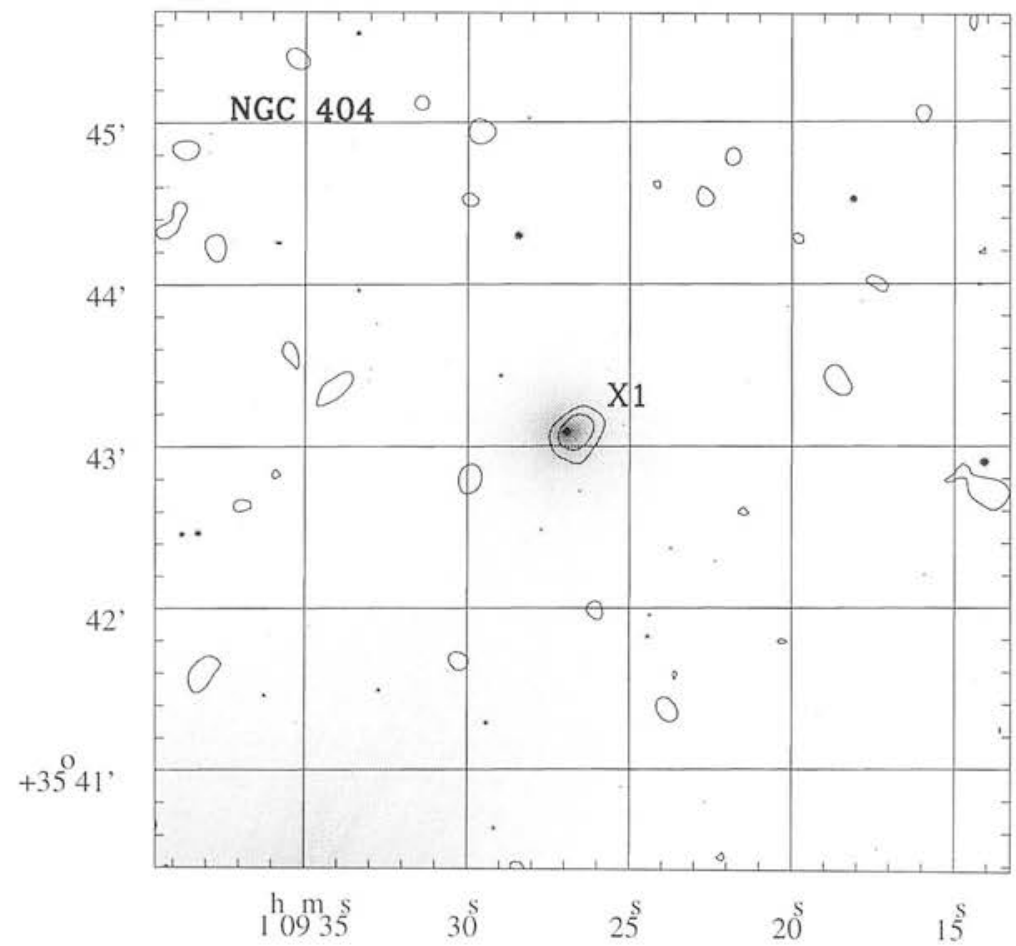

Figure 2.10: NGC 404 X-ray contours overlaid on optical JKT image. ROSAT exposure time $=23562.6$ seconds. 


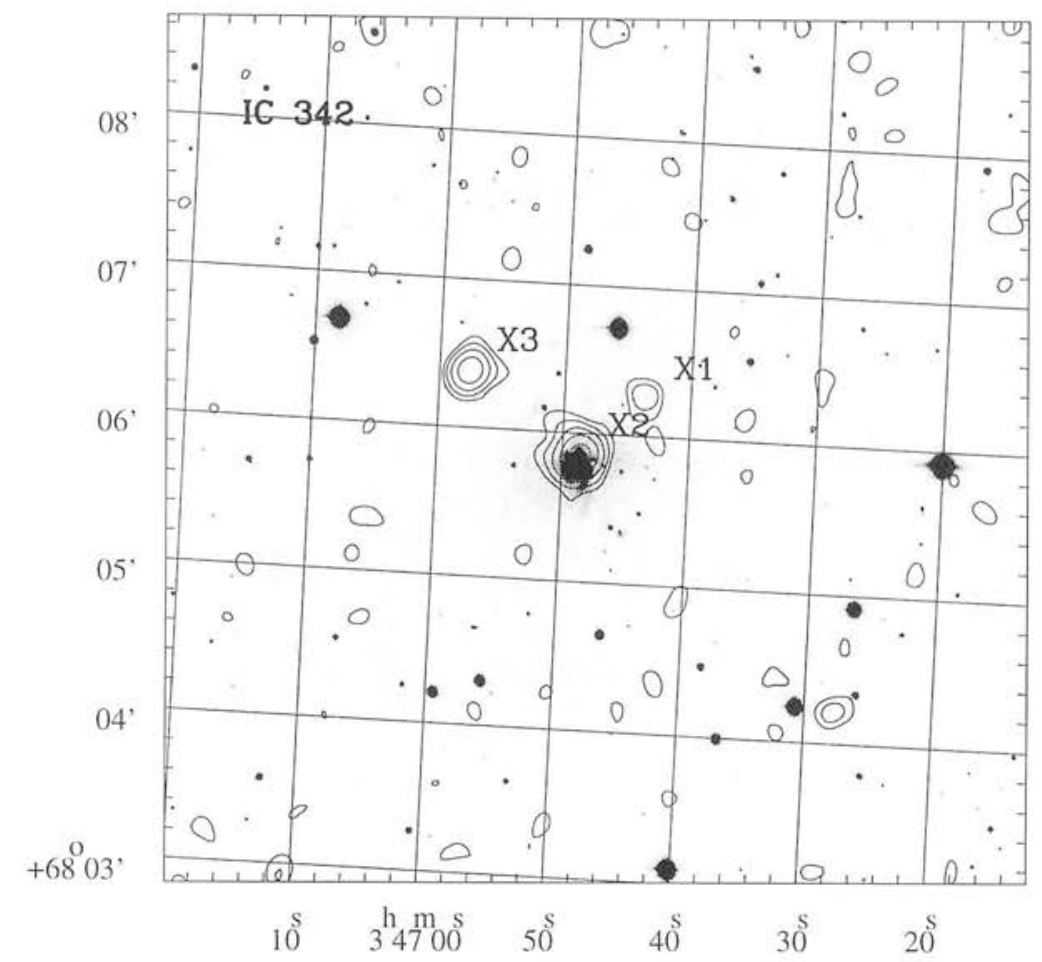

Figure 2.11: IC 342 X-ray contours overlaid on optical JKT image. ROSAT exposure time $=18990.4$ seconds.

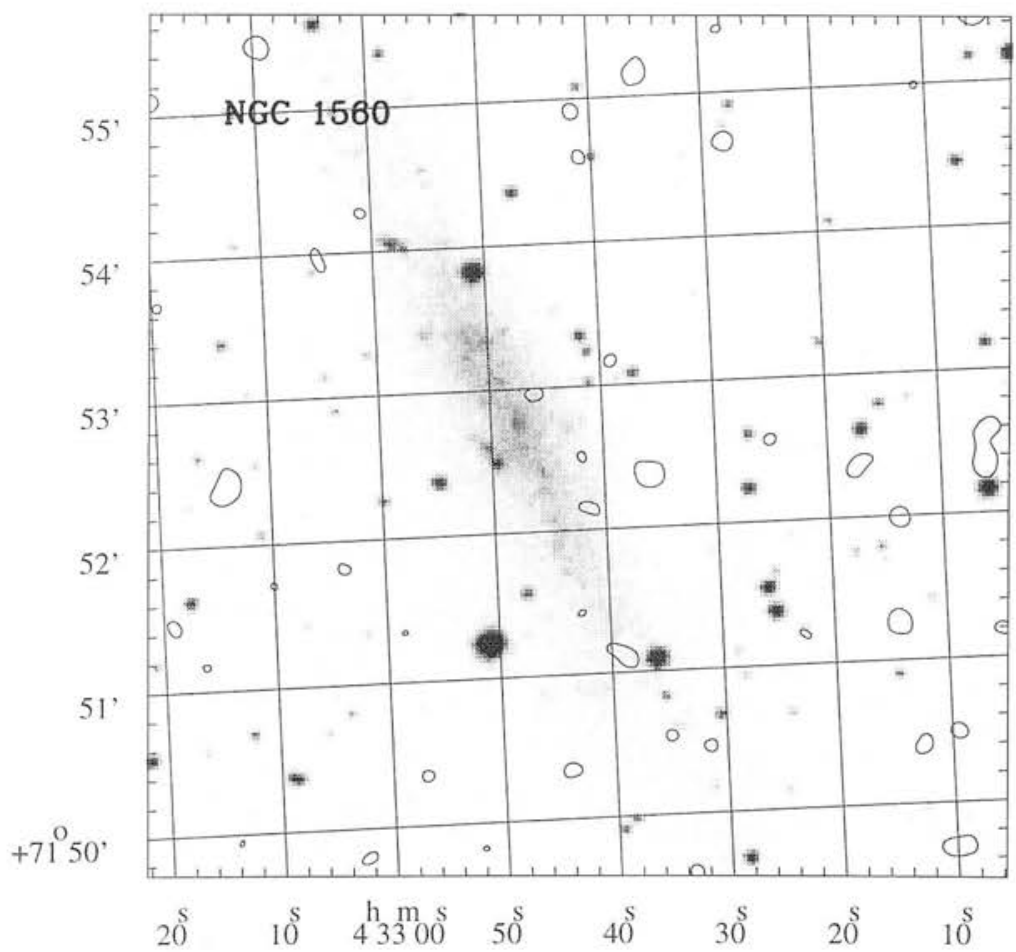

Figure 2.12: NGC1560 X-ray contours overlaid on optical DSS image. ROSAT exposure time $=17287.5$ seconds. 


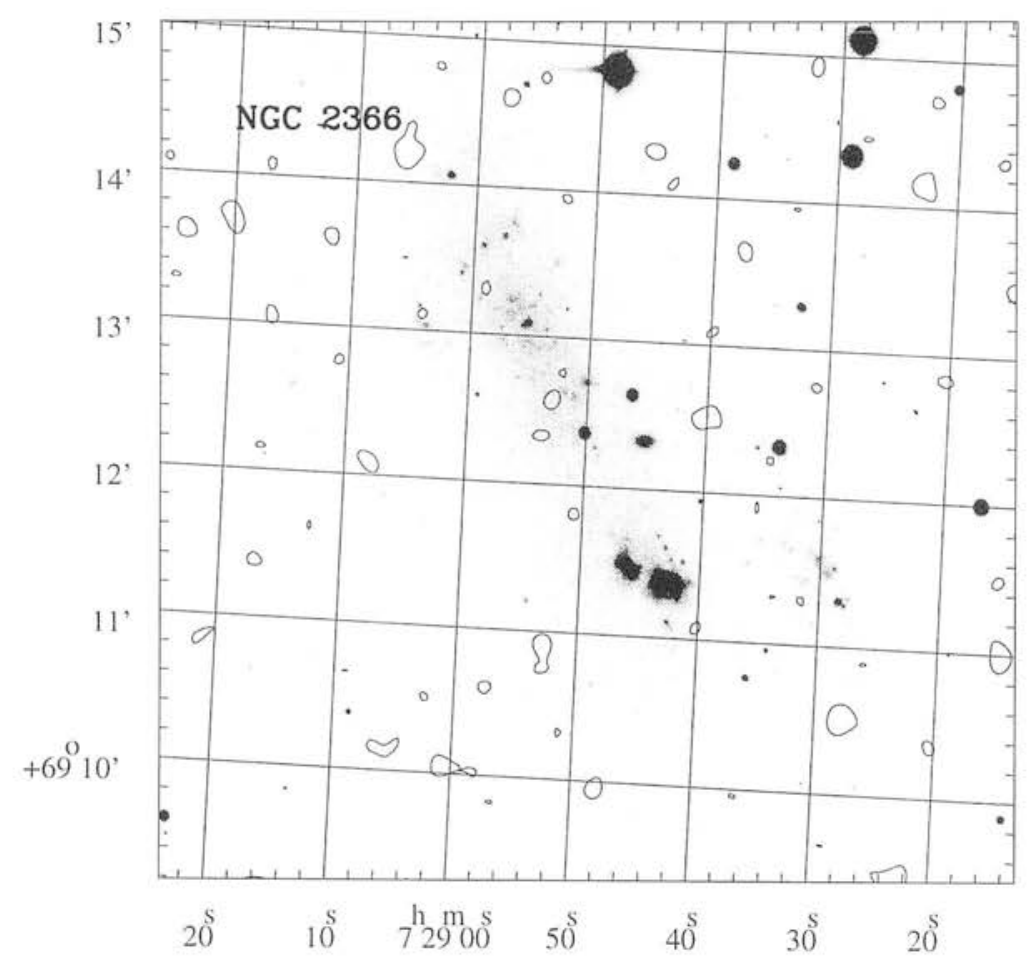

Figure 2.13: NGC 2366 X-ray contours overlaid on optical JKT image. ROSAT exposure time $=31465.5$ seconds.

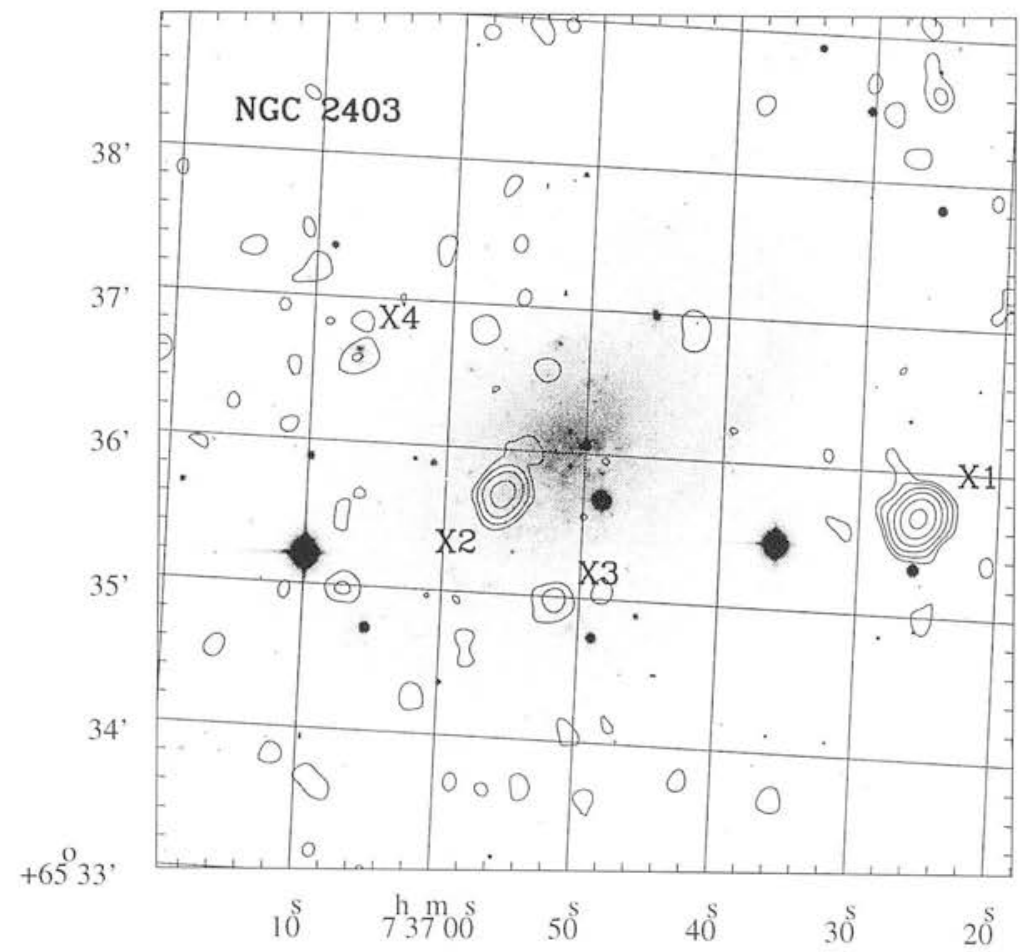

Figure 2.14: NGC 2403 X-ray contours overlaid on optical JKT image. ROSAT exposure time $=26244.7$ seconds. 


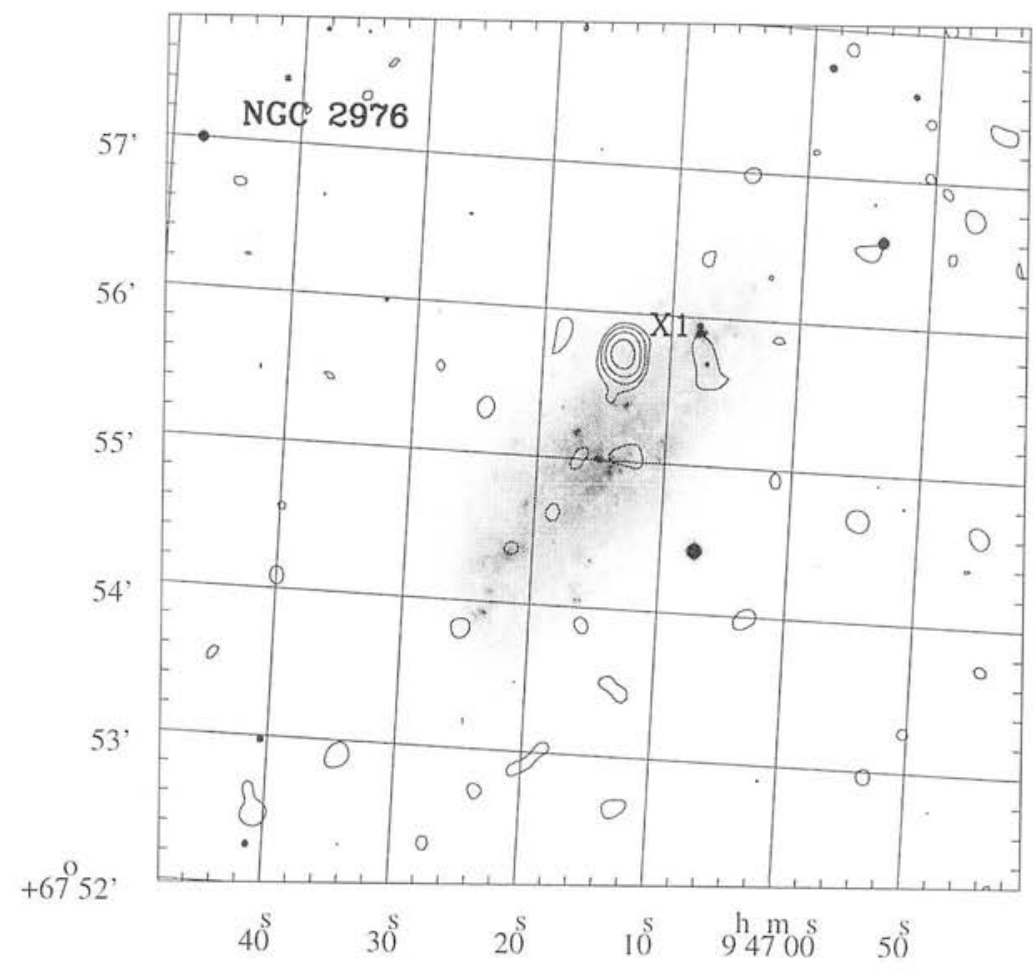

Figure 2.15: NGC 2976 X-ray contours overlaid on optical JKT image. ROSAT exposure time $=49482.3$ seconds.

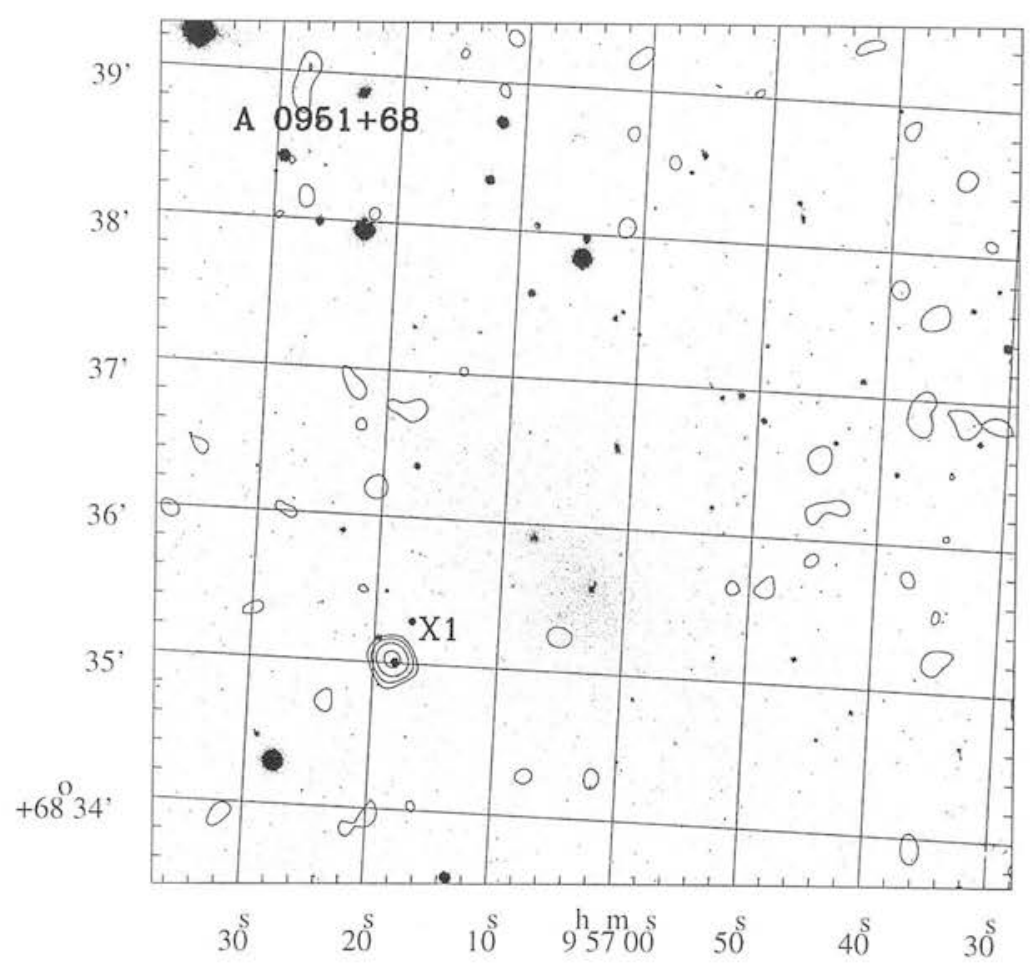

Figure 2.16: A 0951+68 X-ray contours overlaid on optical JKT image. ROSAT exposure time $=14240.2$ seconds. 


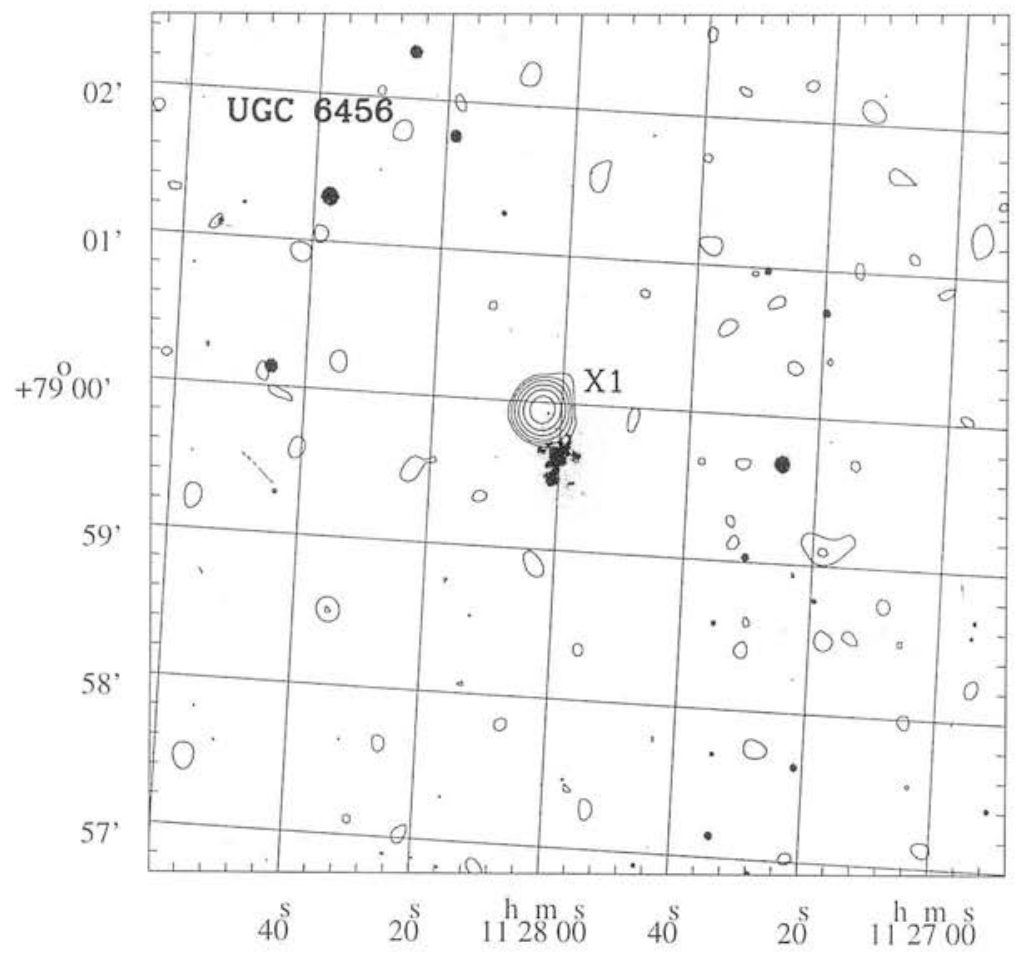

Figure 2.17: UGC 6456 X-ray contours overlaid on optical JKT image. ROSAT exposure time $=13027.7$ seconds.

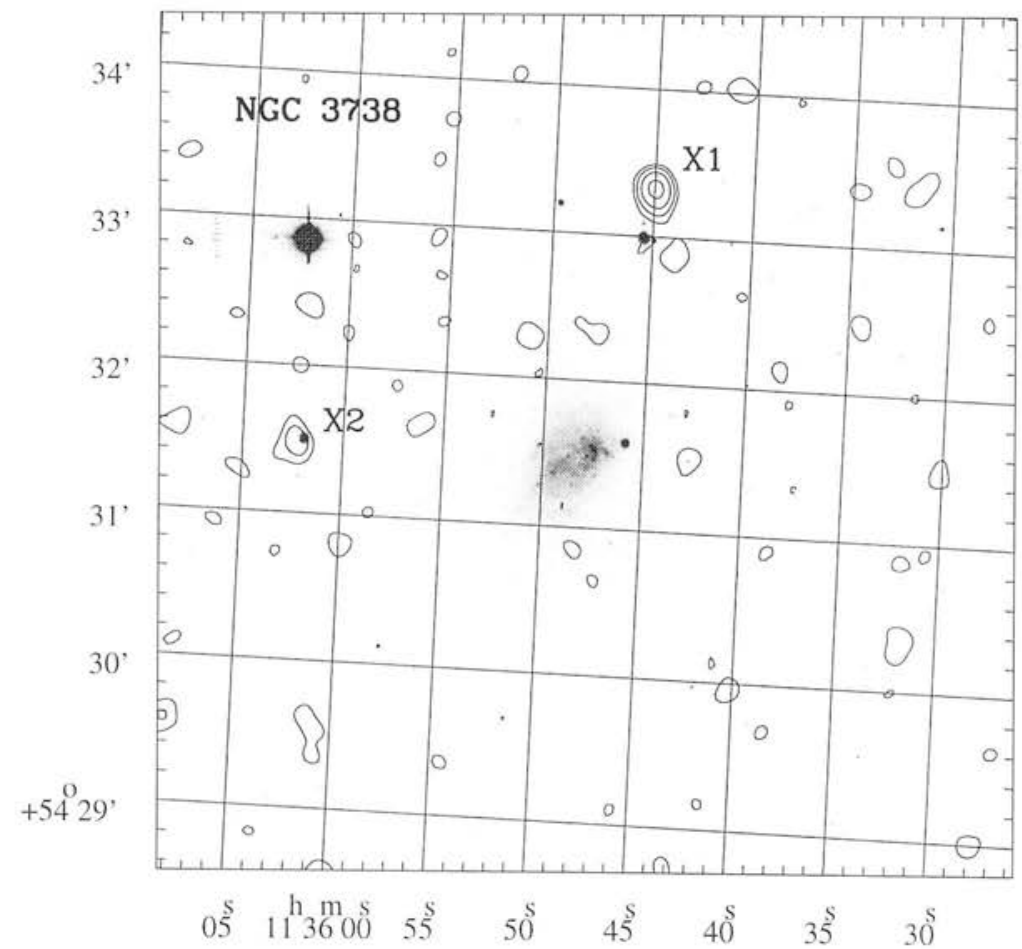

Figure 2.18: NGC 3738 X-ray contours overlaid on optical JKT image. ROSAT exposure time $=13711.2$ seconds. 


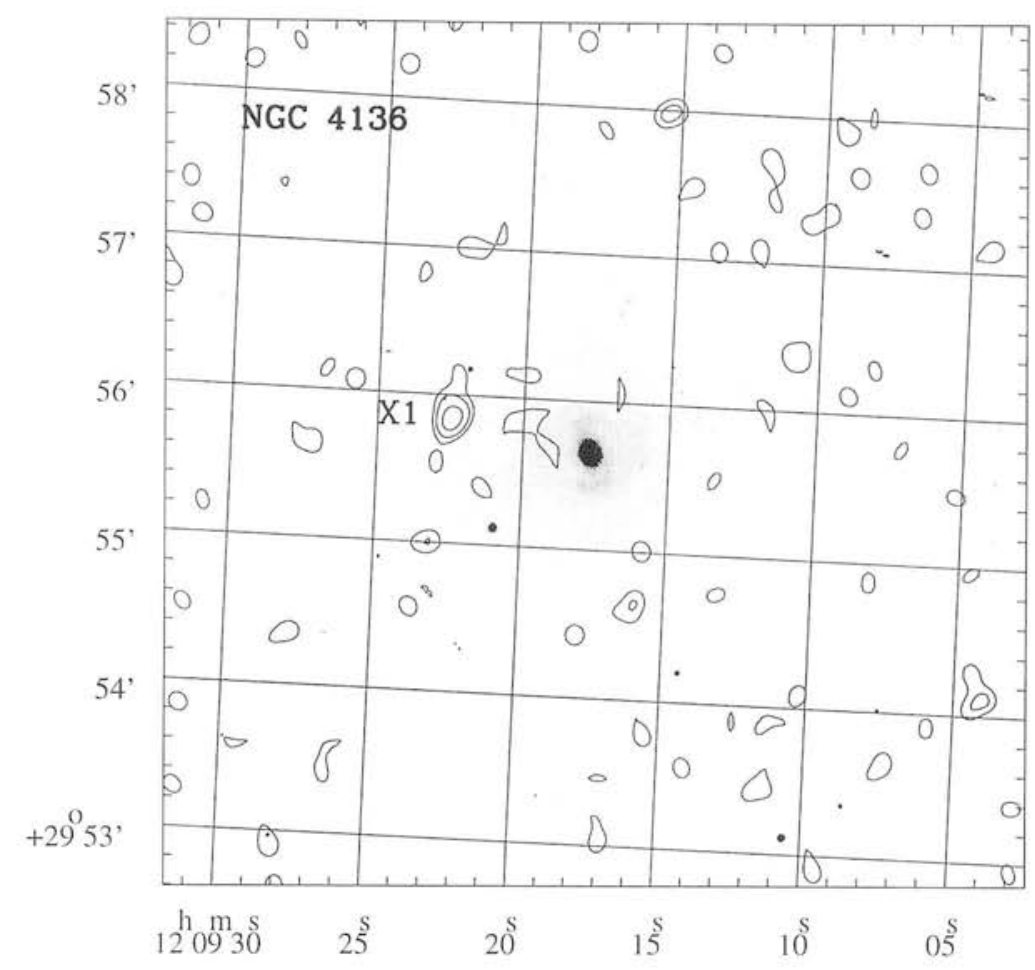

Figure 2.19: NGC 4136 X-ray contours overlaid on optical JKT image. ROSAT exposure time $=1951.0$ seconds.

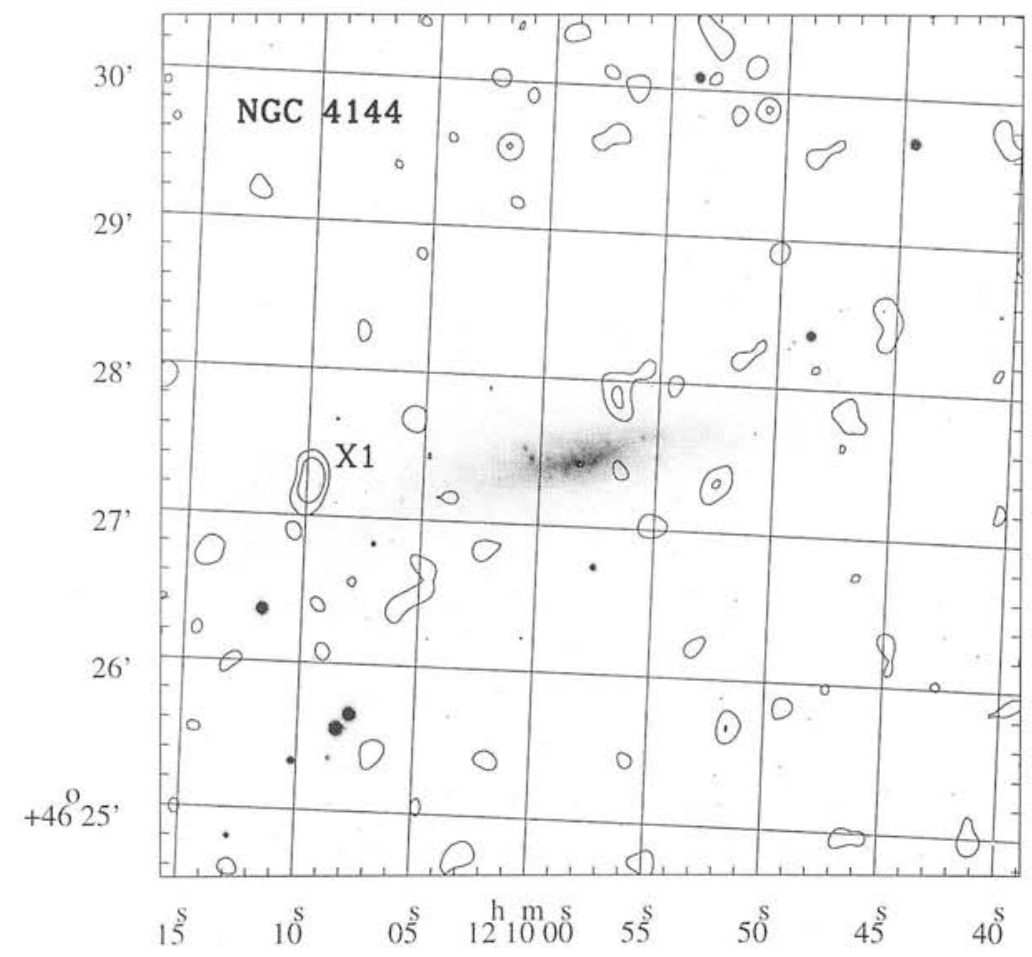

Figure 2.20: NGC4144 X-ray contours overlaid on optical JKT image. ROSAT exposure time $=11083.4$ seconds . 


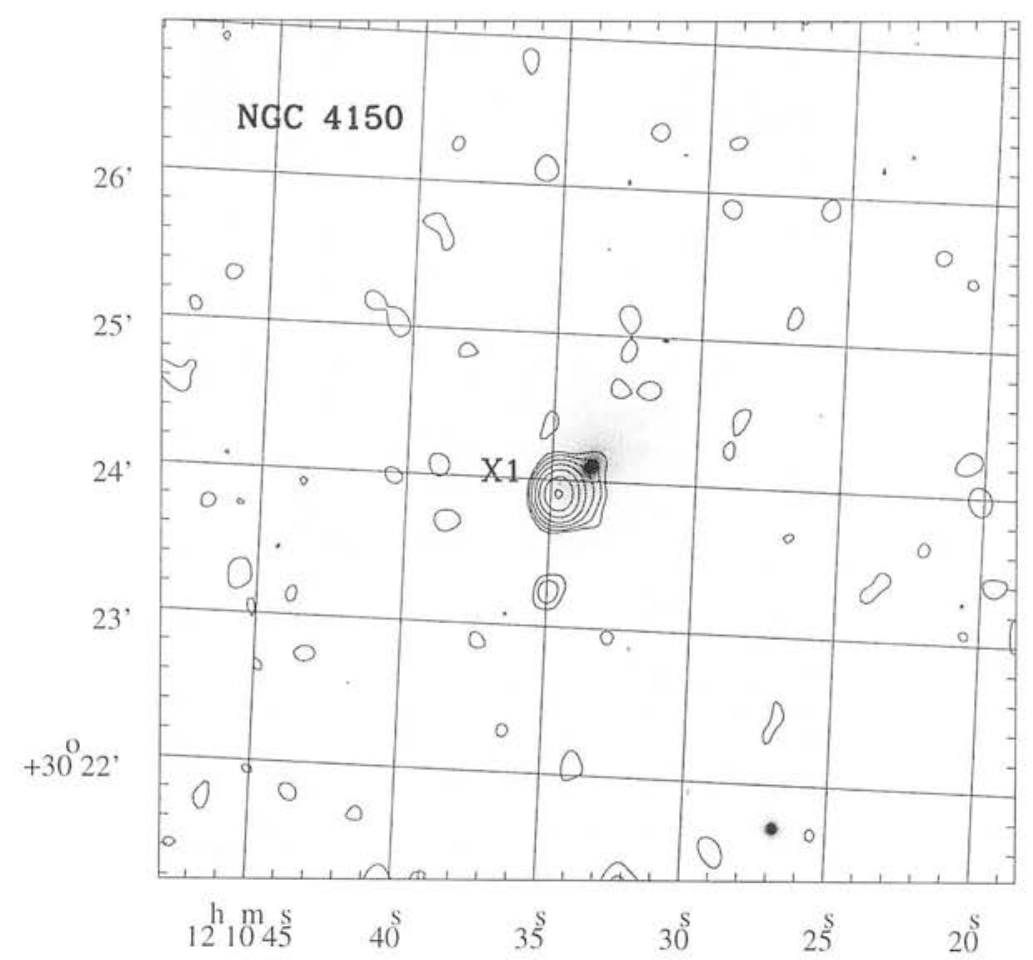

Figure 2.21: NGC4150 X-ray contours overlaid on optical JKT image. ROSAT exposure time $=14083.9$ seconds.

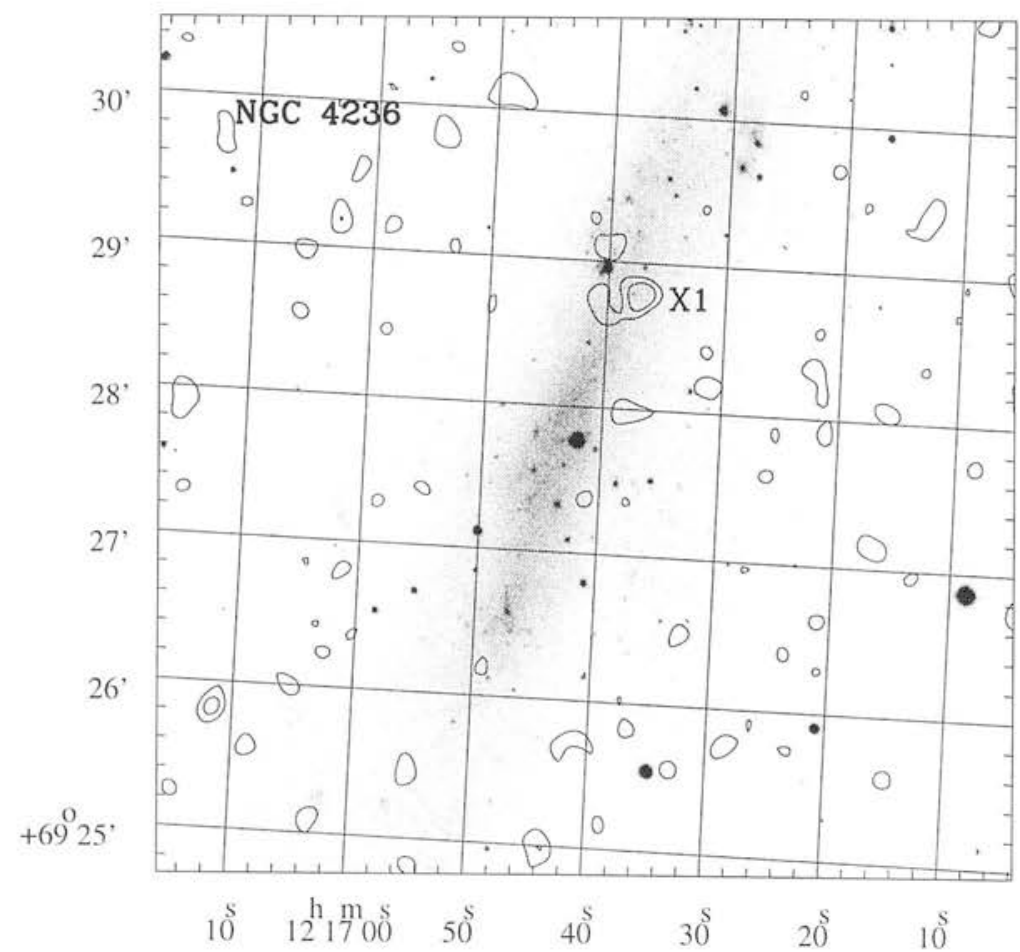

Figure 2.22: NGC 4236 X-ray contours overlaid on optical JKT image. ROSAT exposure time $=10933.8$ seconds. 


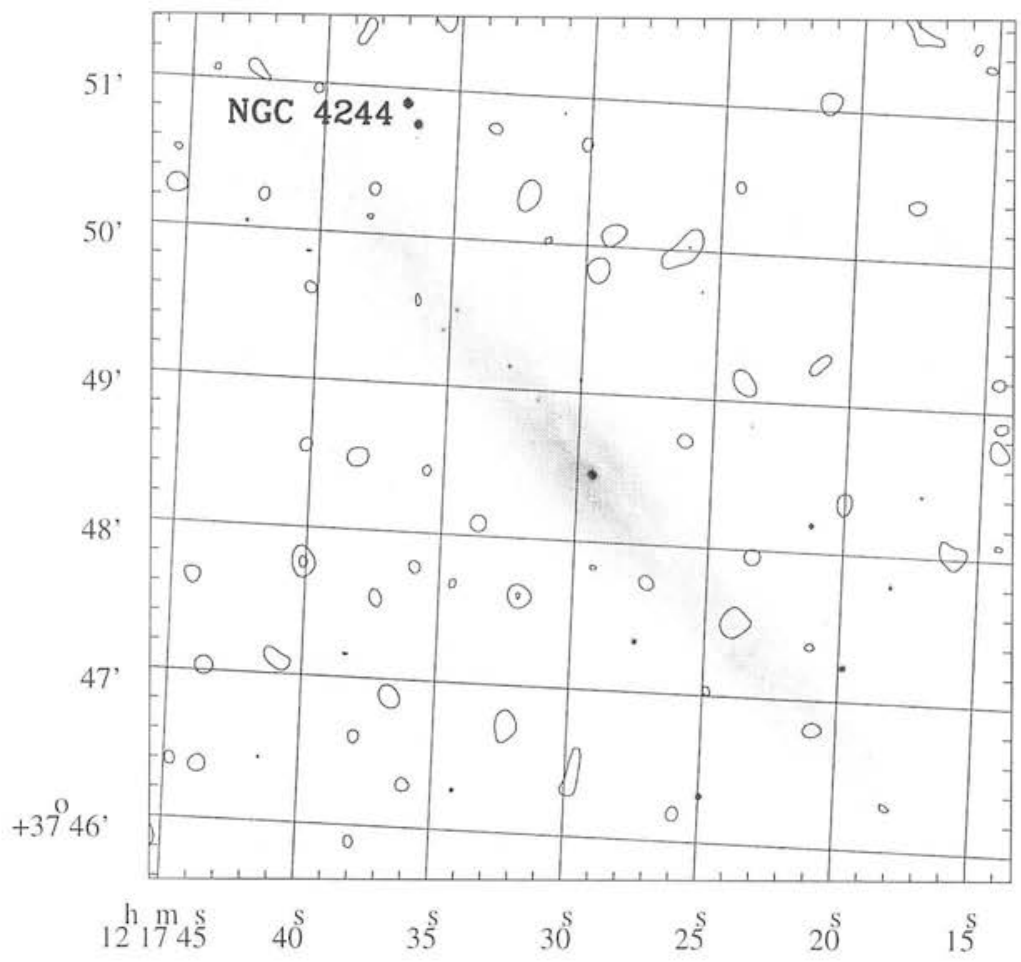

Figure 2.23: NGC $4244 \mathrm{X}$-ray contours overlaid on optical JKT image. ROSAT exposure time $=8630.9$ seconds.

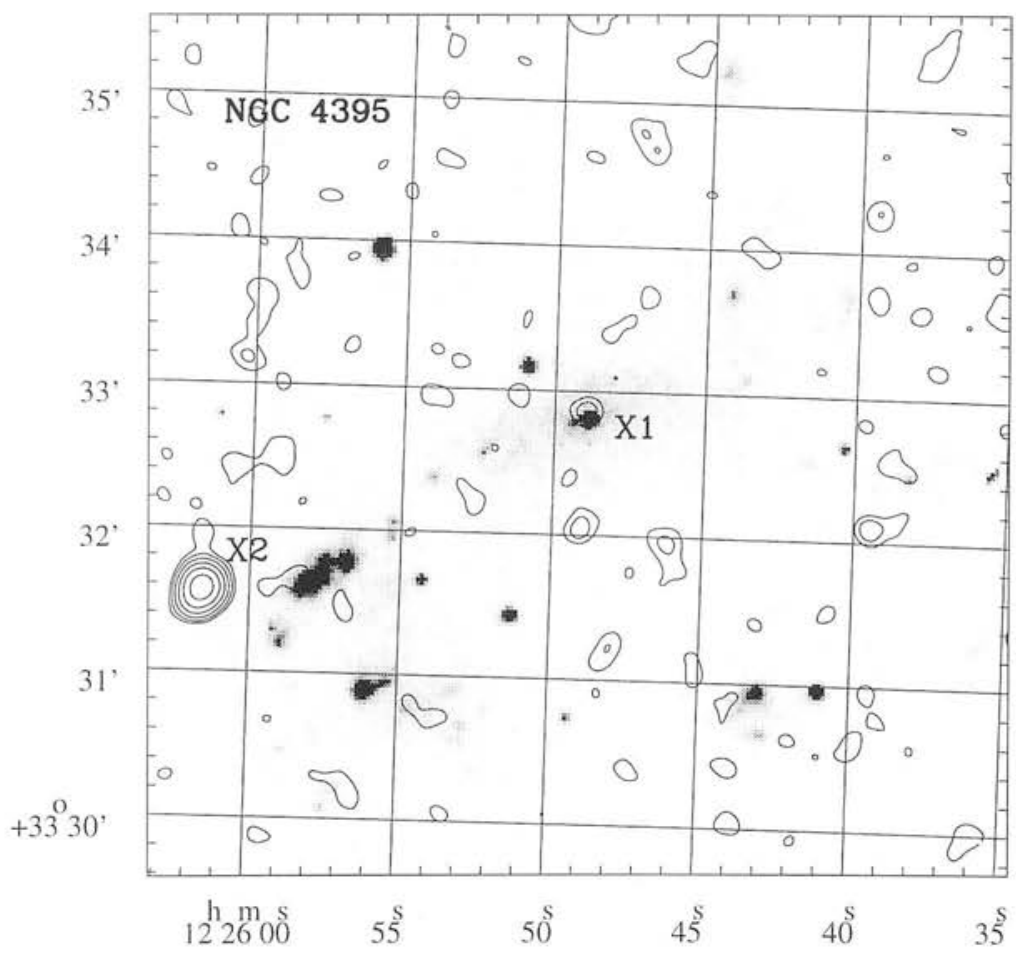

Figure 2.24: NGC 4395 X-ray contours overlaid on optical DSS image. ROSAT exposure time $=11252.5$ seconds. 


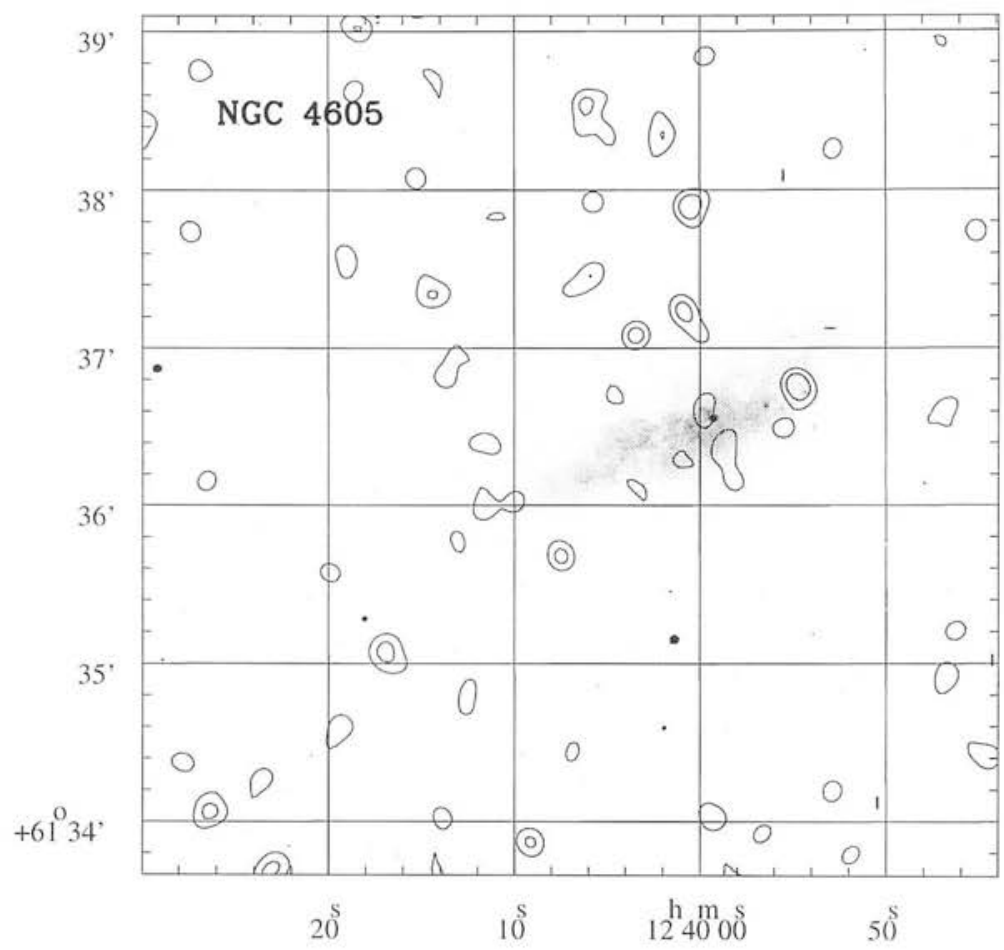

Figure 2.25: NGC 4605 X-ray contours overlaid on optical JKT image. ROSAT exposure time $=2158.4$ seconds.

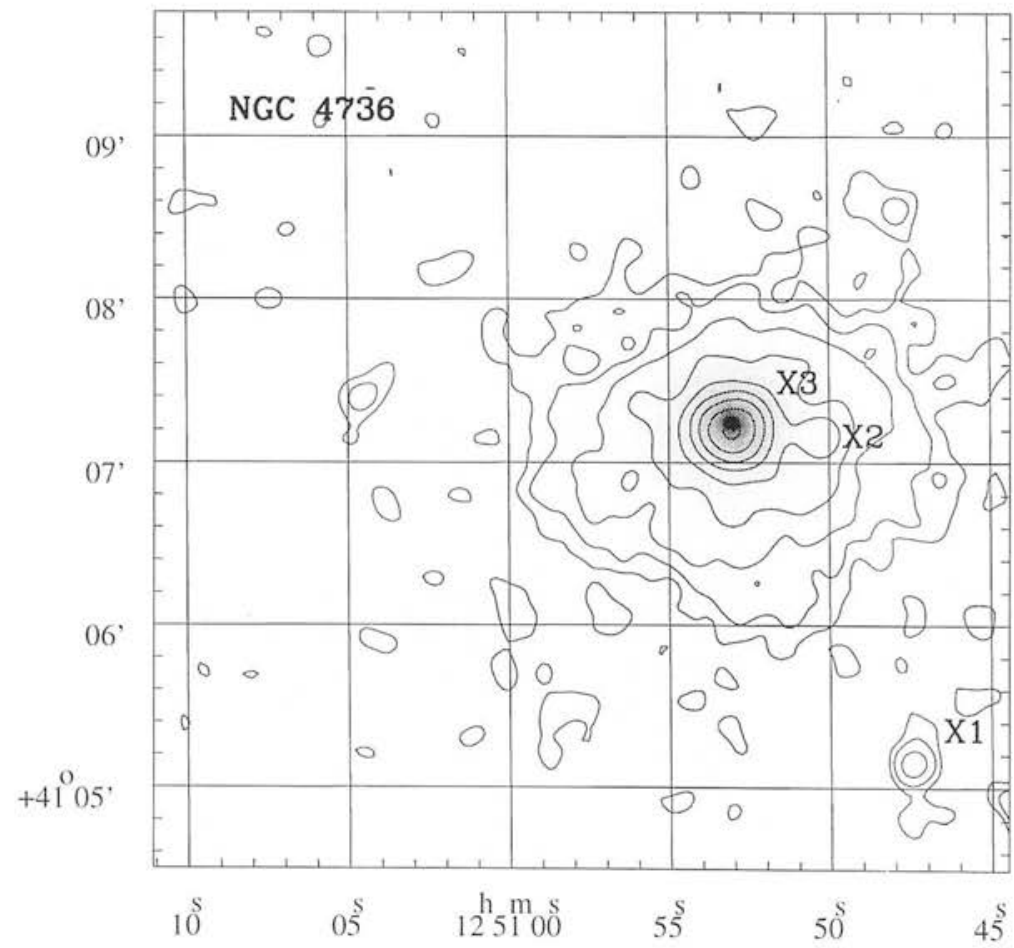

Figure 2.26: NGC 4736 X-ray contours overlaid on optical JKT image. ROSAT exposure time $=138904.4$ seconds. 


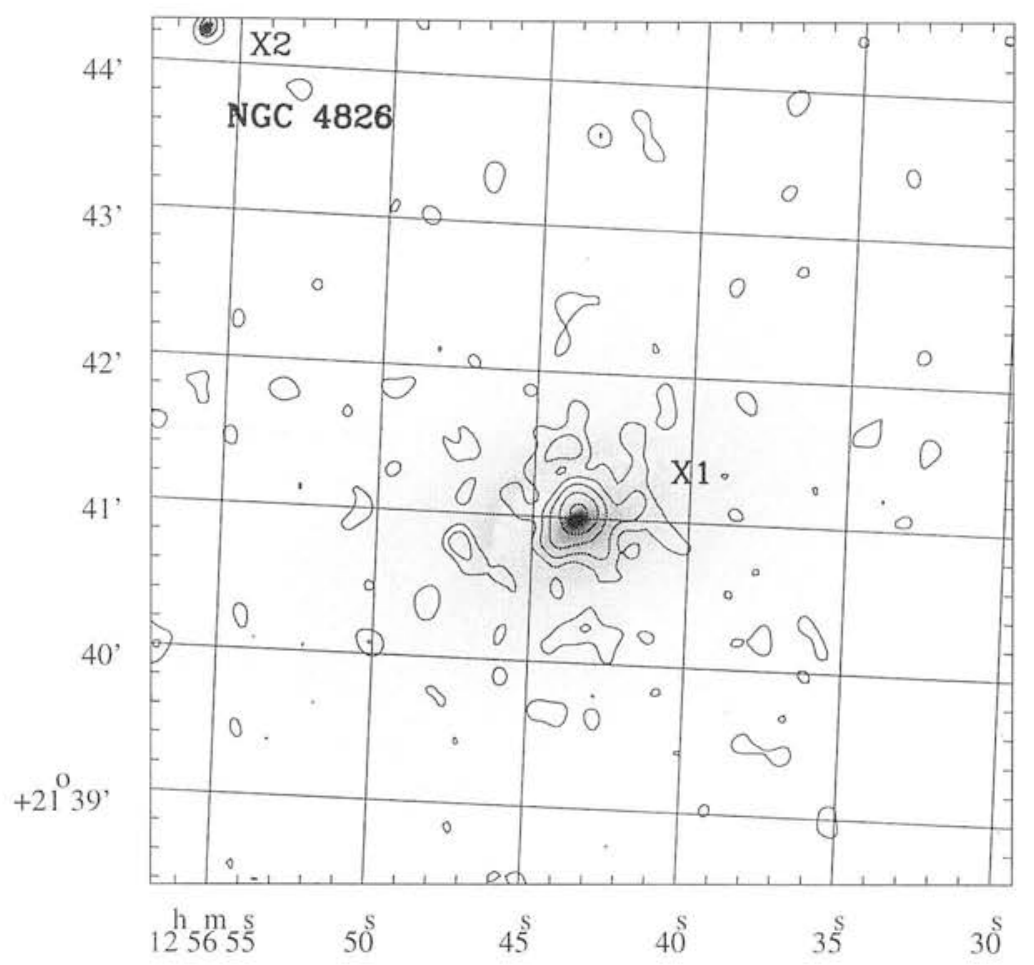

Figure 2.27: NGC $4826 \mathrm{X}$-ray contours overlaid on optical JKT image. ROSAT exposure time $=24633.9$ seconds.

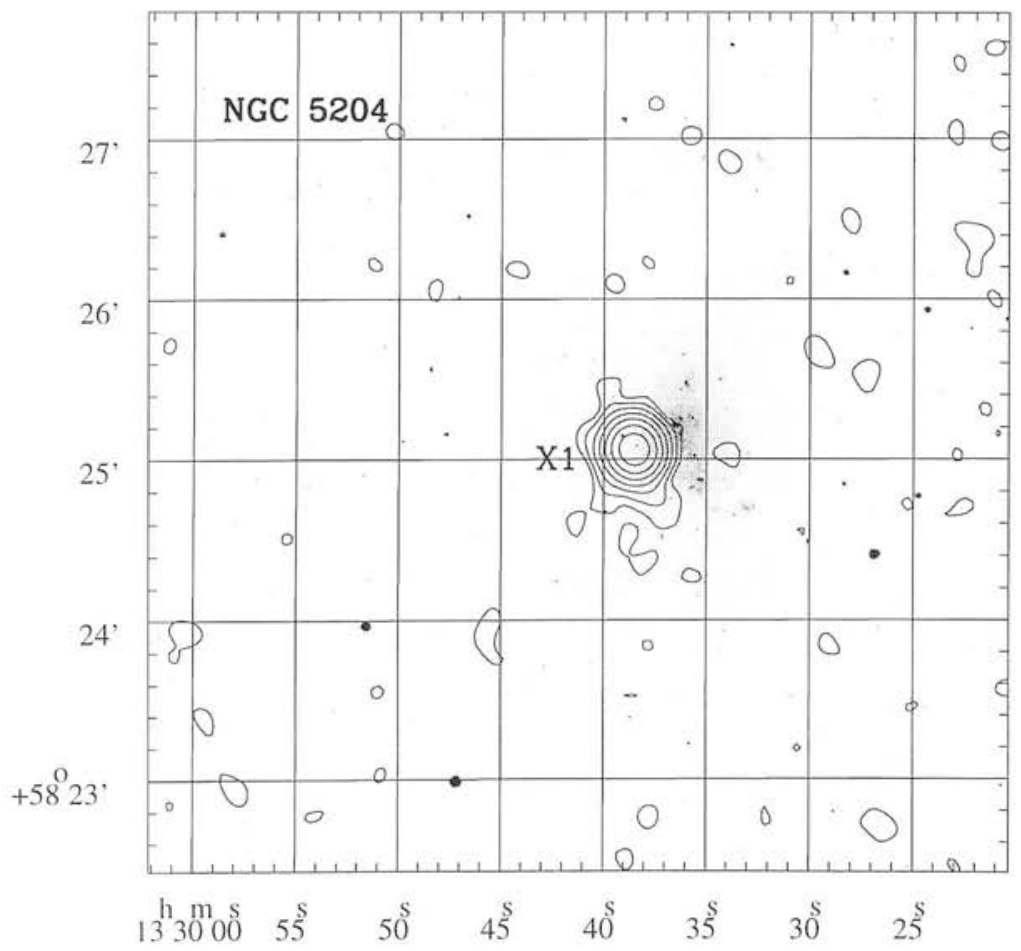

Figure 2.28: NGC 5204 X-ray contours overlaid on optical JKT image. ROSAT exposure time $=28237.1$ seconds. 


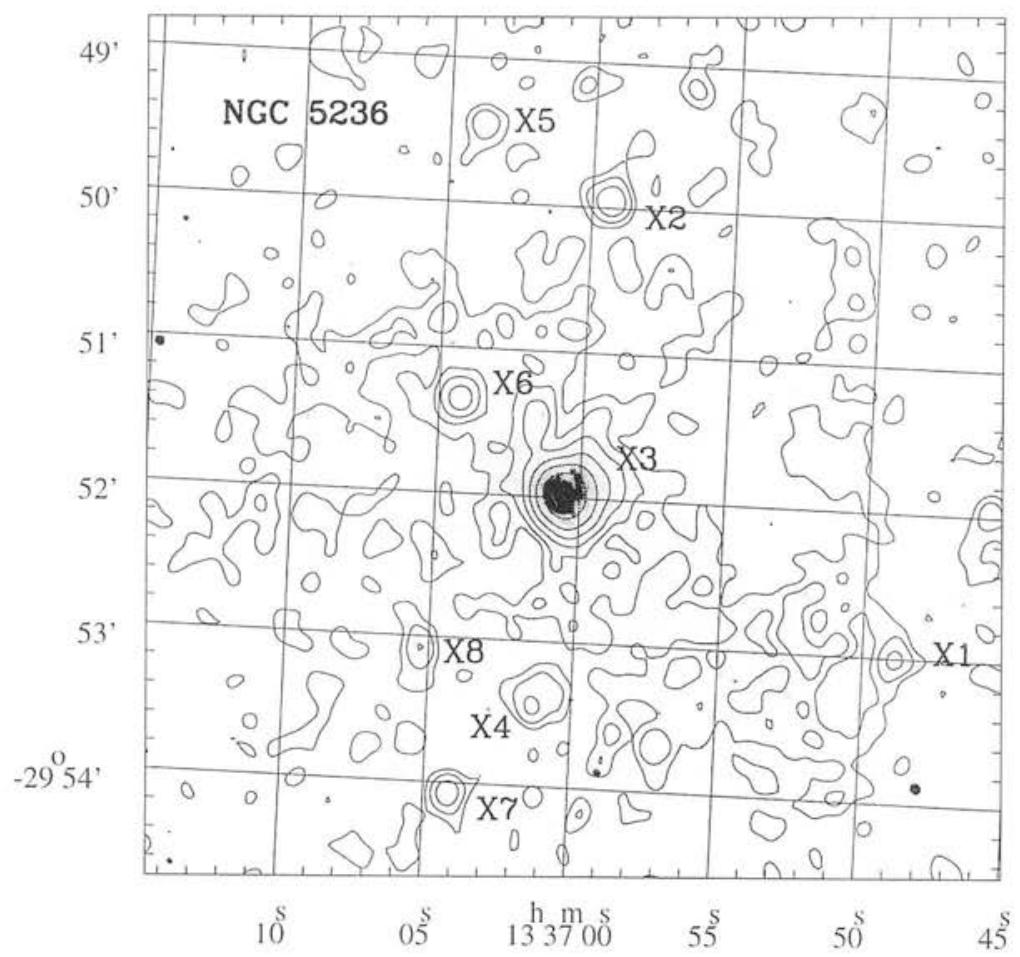

Figure 2.29: NGC5236 X-ray contours overlaid on optical JKT image. ROSAT exposure time $=47237.0$ seconds.

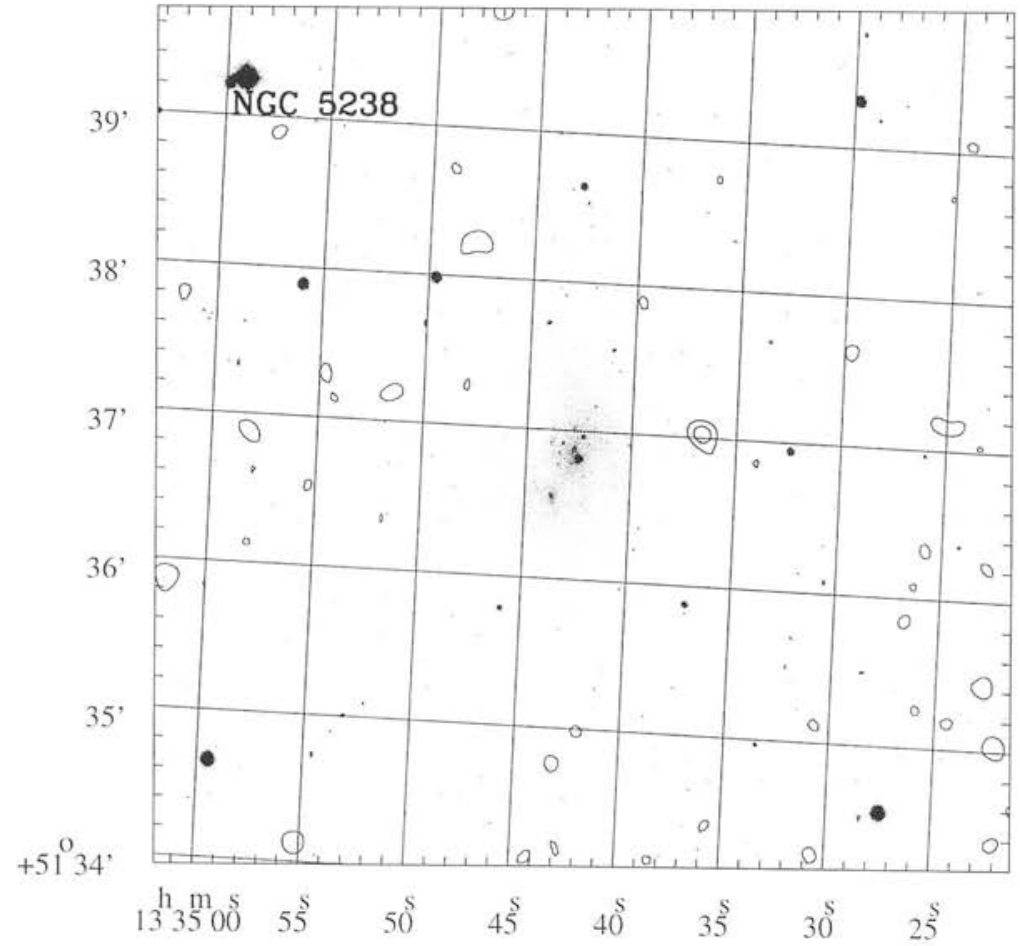

Figure 2.30: NGC 5238 X-ray contours overlaid on optical JKT image. ROSAT exposure time $=23163.1$ seconds. 


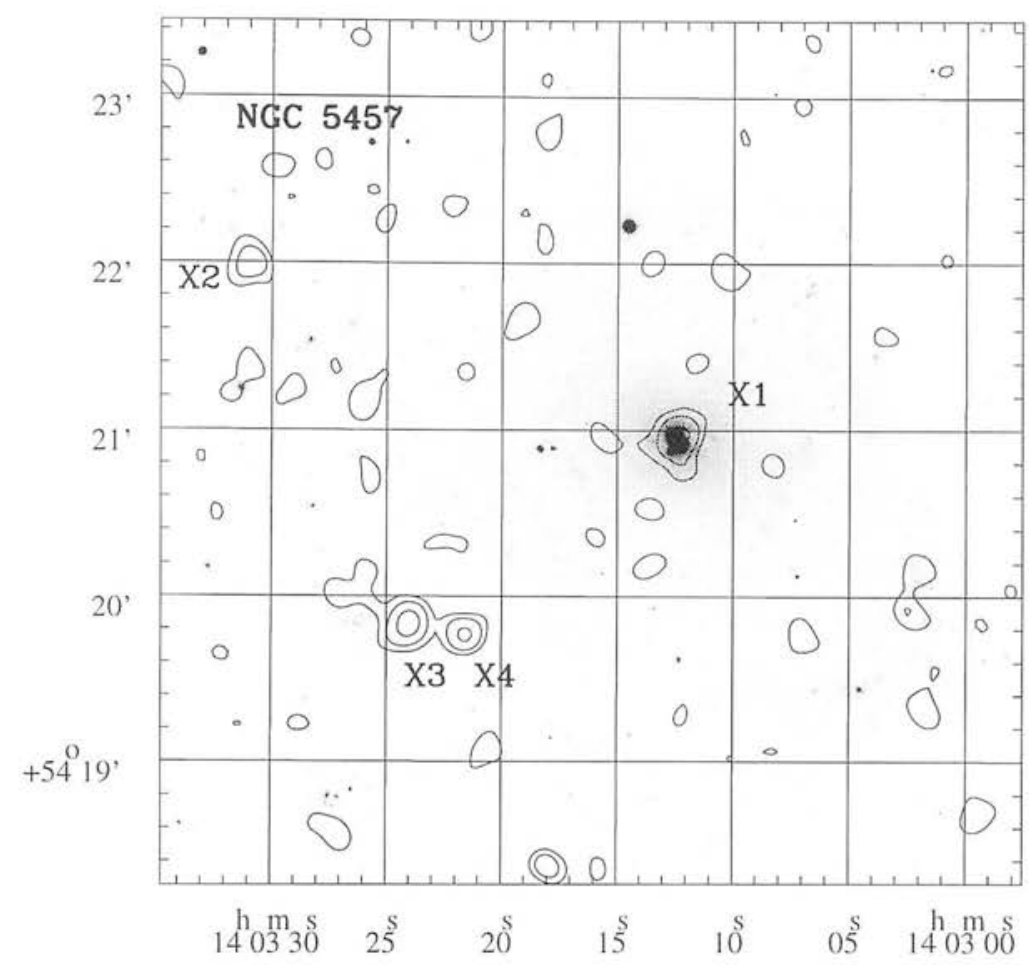

Figure 2.31: NGC 5457 X-ray contours overlaid on optical JKT image. ROSAT exposure time $=50813.9$ seconds.

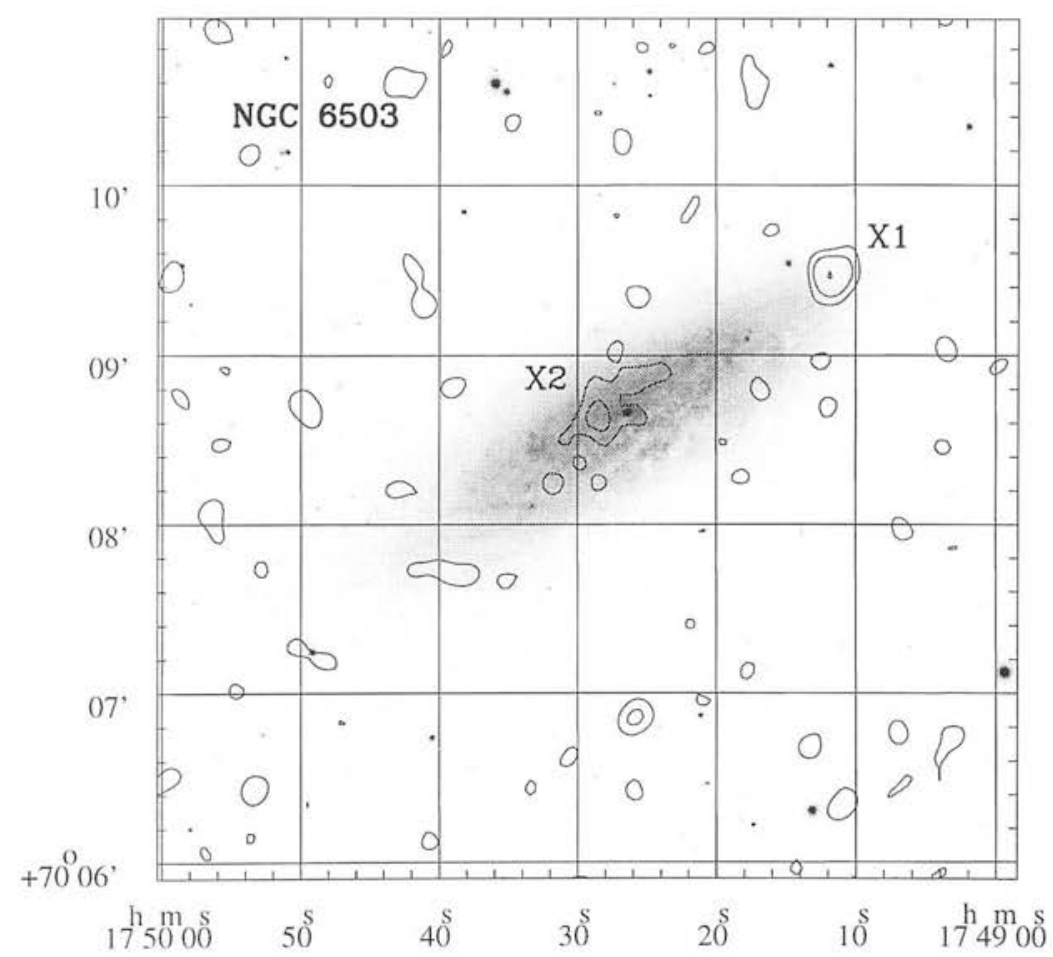

Figure 2.32: NGC 6503 X-ray contours overlaid on optical JKT image. ROSAT exposure time $=14640.6$ seconds. 


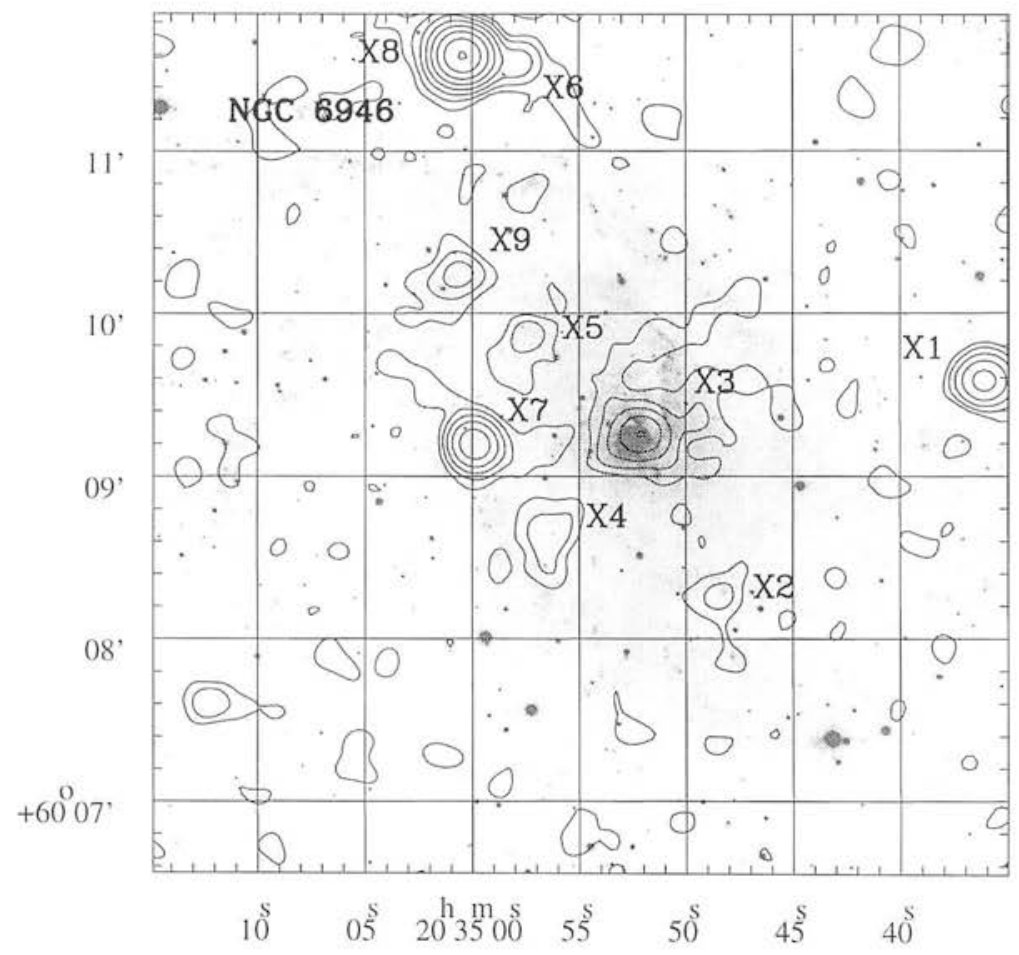

Figure 2.33: NGC 6946 X-ray contours overlaid on optical JKT image. ROSAT exposure time $=81399.7$ seconds. 


\subsection{Notes on individual objects}

A small review on each object with HRI observations will be given in what remains of this chapter. Notes will be given for galaxies in the sample which either have ROSAT data analysed here (objects in table 2.2 ) or have already been reported extensively in the literature (objects in table 2.6). All the objects will be included in the subsequent analysis in chapter 5 .

NGC 147: NGC 147 was observed with the ROSAT HRI and no X-ray emission was detected. These observations have also been reported by Brandt et al. (1997). They give a. $2 \sigma$ upper limit for the flux from a point source located in the galaxy of $6 \times 10^{-14}$ ergs $\mathrm{s}^{-1} \mathrm{~cm}^{-2}$ in the $0.1-2.5 \mathrm{keV}$ band-pass, which is in good agreement with the upper limit reported in table 2.5 .

NGC 185: As with NGC 147, no X-ray emission was detected with the ROSAT HRI for NGC 185. These observations have also been reported by Brandt et al. (1997). A $2 \sigma$ upper limit for the flux of a point source was found to be $4 \times 10^{-14} \mathrm{ergs} \mathrm{s}^{-1} \mathrm{~cm}^{-2}$ in the $0.1-2.5$ $\mathrm{keV}$ band-pass, again in good agreement with the value quoted in table 2.5.

NGC 205: This small elliptical galaxy was observed with the Einstein HRI and no X-ray sources were detected with a flux $>1.8 \times 10^{-12} \mathrm{ergs} \mathrm{s}^{-1} \mathrm{~cm}^{-2}$ in the $0.5-4.0 \mathrm{keV}$ bandpass (Fabbiano, Kim \& Trinchieri 1992; Markert \& Donahue 1985). The ROSAT HRI observations of NGC 205 reported here give an upper limit of $6.78 \times 10^{-14} \mathrm{ergs} \mathrm{s}^{-1} \mathrm{~cm}^{-2}$ for the flux of any point source in the nuclear region. The only source seen in figure 2.7 lies $2.7^{\prime}$ away from the nucleus and is probably not associated with the galaxy.

NGC 221: A strong off-nuclear source in NGC 221 has been found with the HRI on both Einstein and ROSAT. The flux reported here is consistent with the Einstein measurements $\left(F_{X}=9.1 \times 10^{-13} \mathrm{ergs} \mathrm{s}^{-1} \mathrm{~cm}^{-2}\right.$ in the $0.5-4.0 \mathrm{keV}$ band-pass (Fabbiano, Kim \& Trinchieri 1992)). The X-ray source lies $\sim 7$ " away from the NGC 221 nucleus and has no optical counterpart. An upper limit for the nuclear X-ray emission is given in table 2.5. The position of the aperture used to compute the upper limit was shifted slightly from the exact position of the galactic nucleus to avoid contamination from the off-nuclear source.

Dynamical studies of the stellar rotation velocities in this galaxy have revealed the presence of a central dark massive object, probably a black hole, with mass $3 \times 10^{6} M_{\odot}$ (Bender, Kormendy \& Dehnen 1996; Van Der Marel, De Zeeuw \& Rix 1997), which corresponds to an Eddington luminosity of $\sim 10^{44} \mathrm{ergs} \mathrm{s}^{-1}$. The $\mathrm{X}$-ray upper limit in table 2.5 shows 
that the central object is emitting at most at $\lesssim 10^{-8} L_{E d d}$.

NGC 224: No analysis of X-ray data for this galaxy has been done in this thesis. A review of the observed X-ray emission observed has already been given in chapter 1 . A list of the sources found within a radius of $\sim 6^{\prime}$ from the nucleus is shown in table 2.6. The source coincident with the galactic nucleus has been reported to vary (Primini, Forman \& Jones 1993) and is probably an XRB.

NGC 247: Two strong X-ray sources are seen in the southern region of the ROSAT HRI image (see figure 2.9). A $2 \sigma$ upper limit for a point source located in the nuclear region of the galaxy can be found in table 2.5. Zang, Warwick \& Meurs (1997) report the detection of a bright $\left(L_{X}=1.4 \times 10^{38} \mathrm{ergs} \mathrm{s}^{-1}\right)$ ROSAT PSPC source located $\sim 2^{\prime}$ south from the centre of the galaxy. The relative position and luminosity are in good agreement with source X-1 in figure 2.9. Mackie et al. (1995) also report PSPC observations and find a faint nuclear source with $L_{X}=1 \times 10^{36} \mathrm{ergs} \mathrm{s}^{-1}$, which is well below the detection limit of our HRI observations. However, due to the poor spatial resolution of the PSPC, it is not clear whether this source is coincident with the galaxy nucleus.

NGC 253: No analysis of X-ray data for this galaxy has been done in this thesis. This starburst galaxy has very complex X-ray emission. Several point sources have been detected with the Einstein and ROSAT HRI (Fabbiano \& Trinchieri 1984; Vogler \& Pietsch 1999 ) with luminosities of up to a few times $10^{38} \mathrm{ergs} \mathrm{s}^{-1}$ (see table 2.6). The brightest object is variable with a hard spectrum and is probably an XRB (Vogler \& Pietsch 1999). The source located in the nuclear region is extended and does not vary. Extended emission is detected well above and below the galactic plane of this galaxy (see figure 4 in Vogler \& Pietsch 1999) and NGC 253 is a prototype galaxy for the study of X-ray emission from starburst galaxies (see chapter 5). ASCA observations show that two components are required to fit its spectrum in the $2-10 \mathrm{keV}$ energy band: a hard component with $k T \sim 9$ $\mathrm{keV}$ and a soft component with $k T \sim 1 \mathrm{keV}$ (Ptak et al. 1997).

NGC 404: A weak X-ray nuclear source $\left(F_{X}=7.5 \times 10^{-12} \mathrm{ergs} \mathrm{s}^{-1}\right)$ has been detected by the ROSAT HRI in the LINER nucleus of this galaxy. An ASCA 2-10 keV upper limit of $3 \times 10^{-13} \mathrm{ergs} \mathrm{s}^{-1} \mathrm{~cm}^{-2}$ has been reported by Maoz et al. (1998), which implies that the ROSAT flux is either dominated by very soft emission, or that the source is variable. The former idea is supported by recent UV observations of the NGC 404 nucleus which show that the spectrum is dominated by stellar absorption features from massive young stars (Maoz et al. 1998) and not by the blue, featureless continum expected from an active nucleus. 
NGC 598: No analysis of X-ray data for this galaxy has been done in this thesis. Several point sources have been detected with the Einstein and ROSAT HRI in this spiral galaxy. Those sources located within the central $\sim 6^{\prime} \times 6^{\prime}$ region are listed in table 2.6. The most striking source is the nucleus, with a luminosity of $\gtrsim 10^{39} \mathrm{ergs} \mathrm{s}^{-1}$ which makes it a good AGN candidate. However, the nucleus is not detected at radio wavelengths and shows very little line emission in the optical (see Schulman \& Bregman 1995 and chapter 3 of this thesis). ASCA observations show that the emission from this source can be fitted by a soft thermal ( $k T=2.0 \mathrm{keV}$ ) spectrum (Takano et al. 1994) and, with the lack of radio emission, almost certainly exclude the possibility of an active nucleus in NGC 598 . Diffuse emission around the nucleus has been detected in ROSAT HRI as well as PSPC observations (Schulman \& Bregman 1995; Long et al. 1994).

IC 342: Bregman, Cox \& Tomisaka (1993), using ROSAT HRI observations, found evidence for a diffuse nuclear component that could be explained as a hot interstellar medium generated by a very young nuclear starburst. Figure 2.11 shows that three sources lie within the limits of the optical JKT image of the galaxy.

The brightest source is coincident with the nucleus and visual inspection of the image suggests that it is marginally resolved. A comparison of the azimuthally averaged profile of the source and the HRI model PSF can be seen in figure 2.34 and a clear deviation from the model PSF is visible at a radius of between $7^{\prime \prime}$ and $13^{\prime \prime}$ from the centre. For comparison, an off-nuclear source is also shown, which is in good agreement with the model PSF, confirming the diffuse nuclear component.

The other two sources in the figure do not have obvious optical counterparts. Source X1 has a S/N of 2.3 (see table 2.4), but is included here because it was reported by Bregman, Cox \& Tomisaka (1993) (they found a S/N of 2.8, probably due to a different background estimate).

Fabbiano \& Trinchieri (1987) analysed Einstein IPC observations of the nuclear region in IC 342 and argued that the emission is consistent with starburst activity. Unfortunately, the IPC was not able to resolve the three sources detected with the HRI. Recent ASCA observations show that the emission in the $0.2-10 \mathrm{keV}$ band is consistent with a massaccreting compact object (a neutron star or stellar-mass BH) whose X-ray emission is boosted and beamed due to the presence of a relativistic jet (Okada et al. 1998).

NGC 1560: This galaxy was observed for the first time in X-rays and no emission was detected in the ROSAT HRI data. A $2 \sigma$ upper limit for a point source located in the 

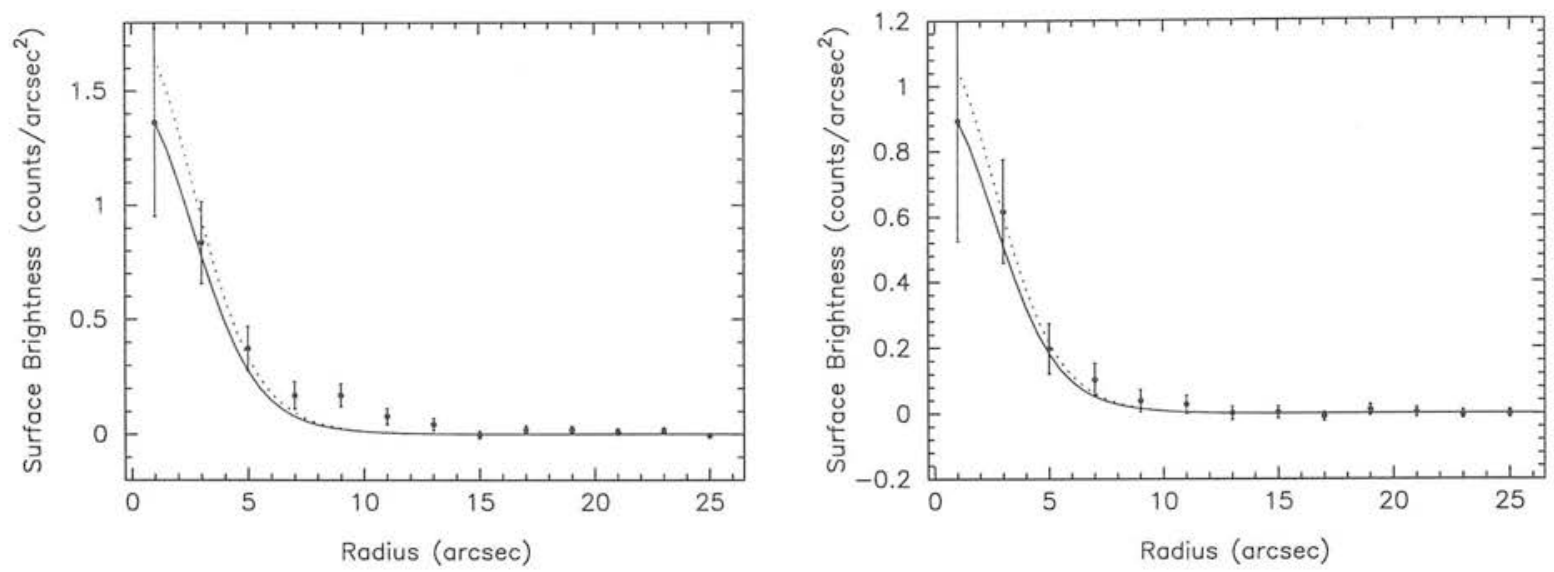

Figure 2.34: Observed profile and model PSF for IC 342 X-2 and an off-nuclear source. The solid line corresponds to a model PSF (equation 2.2) scaled to match the peak of the observed profile. The dashed line corresponds to the same model PSF but with a scale factor found by minimizing a chi-squared fit to the data.

nuclear region of the galaxy can be found in table 2.5 .

NGC 2366: No emission was detected in the ROSAT HRI observations of this galaxy. A $2 \sigma$ upper limit for a point source located in the nuclear region of the galaxy can be found in table 2.5 .

NGC 2403: This galaxy was observed with the Einstein HRI and IPC instruments by Fabbiano \& Trinchieri (1987). Three prominent sources were identified in the IPC observations with no emission from the nuclear region of the galaxy.

The ROSAT HRI observations reported here show a total of 4 point sources associated with the galaxy.

Sources X1 and X3 in figure 2.14 correspond to two of the sources reported by Fabbiano \& Trinchieri (1987) (their third source lies outside the JKT image). It is confirmed that no nuclear source is present in the galaxy and a $2 \sigma$ upper limit for a nuclear point source is given in table 2.5 .

A search for SNRs in NGC 2403 has yielded 35 detections (Matonick et al. 1997). The position of remnant number 15 in table 2 of Matonick et al. (1997) is coincident with the X-ray source X-3 reported here (see table 2.4). A very faint optical counterpart is observed at this position. If the identification is correct the SNR would belong to a class of super-luminous (probably young) remnants (Schlegel 1994b).

Source X4 corresponds to a giant HII region. A photometric study of this region (N2403A) reveals more than 1400 detected stars, among them 800 O-type stars and a lower limit 
NGC 2976: This galaxy was observed for the first time in X-rays and no emission was detected in the ROSAT HRI data. A $2 \sigma$ upper limit for a point source located in the nuclear region of the galaxy can be found in table 2.5.

A 0951+68: This galaxy has been observed in X-rays for the first time. As can be seen in figure 2.16, the only detected source is probably a foreground or background object.

NGC 3031: No analysis of X-ray data for this galaxy has been done in this thesis. Nine point sources have been detected in the nucleus of this low-luminosity AGN from observations with the Einstein HRI (Fabbiano 1988a). Those sources located within the central $\sim 6^{\prime} \times 6^{\prime}$ region are listed in table 2.6. The emission is dominated by the nuclear source, with a luminosity $\lesssim 10^{40}$ ergs s$^{-1}$ in the $0.2-4.0 \mathrm{keV}$ band-pass. Einstein IPC data show that the spectrum of the nuclear source is soft, with a good fit given by thermal emission with $k T \sim 1 \mathrm{keV}$ or by a power law with index $\alpha \sim 2$ (Fabbiano 1988a). This result, however, has been questioned by ASCA observations which are consistent with a nuclear power law distribution with index $\alpha \sim 1$ in the $0.2-10 \mathrm{keV}$ energy band (Serlemitsos, Ptak \& Yaqoob 1996). X-ray long term and fast variability by significant factors has been reported for the nuclear source (Petre et al. 1993; Ishisaki et al. 1996; Serlemitsos, Ptak \& Yaqoob 1996).

Leo B: No analysis of X-ray data for this galaxy has been done in this thesis. Leo B was observed with the Einstein HRI. Markert \& Donahue (1985) report that no sources were detected.

UGC 6456: This is a blue compact galaxy. These galaxies are characterized by low metallicities, high gas content and vigorous star formation. In UGC 6456, evidence of both a recent episode of strong star formation (600-700 Myr) and an older stellar population has been detected (Lynds et al. 1998).

The JKT optical images show that the galaxy has numerous bright knots of emission surrounded by a low surface brightness outer envelope (Johnson 1997). The knots of emission are displaced south from the geometrical center of the envelope and it is not clear whether they represent the true nuclear region of the galaxy (figure 2.35).

ROSAT PSPC observations of this galaxy show a central X-ray core and three extended structures connected to the central source (Papaderos et al. 1994). This morphology was interpreted as outflows from the central region of the galaxy, powered by starburst activity. 


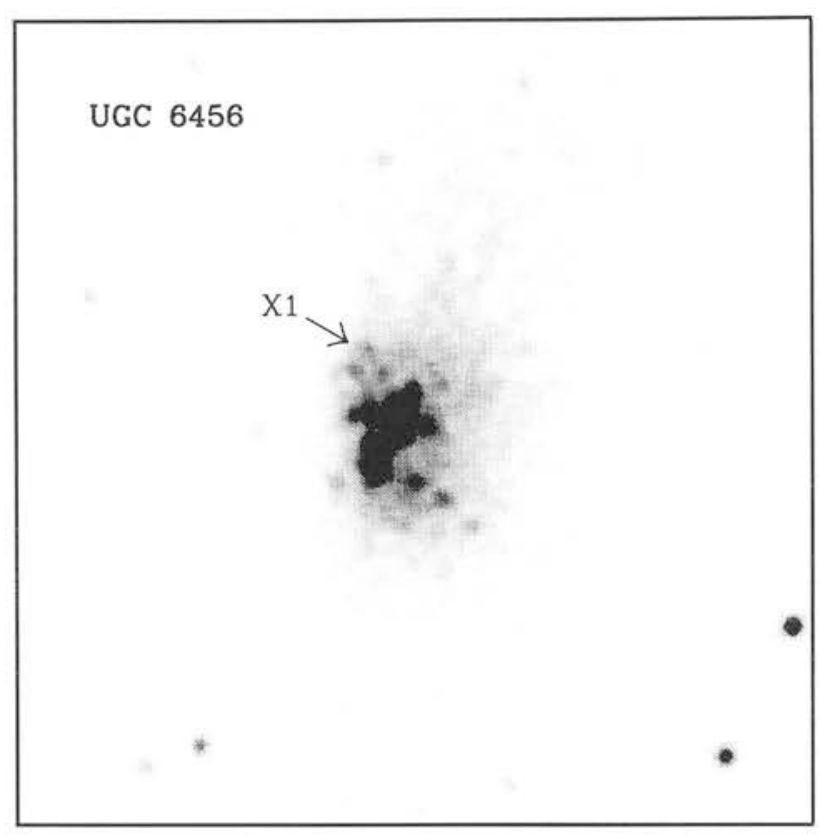

Figure 2.35: Optical I-band image of UGC 6456. The position of the optical counterpart for the X-ray source X1 is indicated.

The total PSPC flux within a circular aperture of radius $3^{\prime}$ is $1.6 \times 10^{-13} \mathrm{ergs} \mathrm{s}^{-1} \mathrm{~cm}^{-2}$.

The high resolution data reported here show a strong X-ray source located at the northmost limit of the optical emission knots (but displaced to the west with respect to the the geometrical center of the outer envelope - see figure 2.35). There is no evidence of extended X-ray emission in the observations, probably due to the lower sensitivity of the HRI. The HRI flux of the point source is $\sim 9 \times 10^{-13} \mathrm{ergs} \mathrm{s}^{-1} \mathrm{~cm}^{-2}$, about 5 times more luminous than the PSPC observations. A very luminous XRB $\left(L_{X} \gtrsim 10^{38}\right.$ ergs s$\left.^{-1}\right)$ could be responsible for this flux variation.

NGC 3738: This galaxy has an irregular optical appearance with several bright knots of emission, although the outer parts are quite regular. None of the bright knots seems to coincide with the geometrical center of the galaxy (Johnson 1997).

NGC 3738 has been observed in X-rays for the first time and no point sources associated with the galaxy have been found. An upper limit for the X-ray emission can be found in table 2.5. Source X1 in figure 2.18 is coincident with a faint knot of optical emission and is probably a foreground or background object. Source X2 is coincident with a bright point-like object and probably corresponds to a foreground star.

NGC 4136: This spiral galaxy has been observed for the first time in X-rays. No sources associated with the nuclear region have been found. An upper limit to the flux from a 


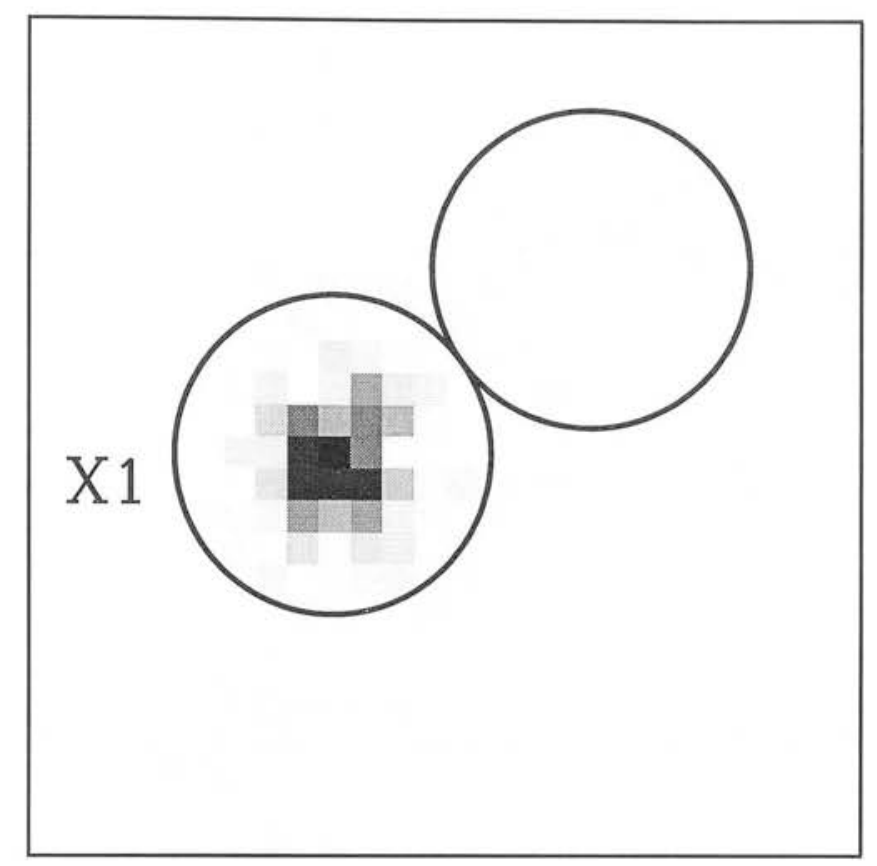

Figure 2.36: X-ray raw image of NGC4150 (pixel size $\left.=2^{\prime \prime} \times 2^{\prime \prime}\right)$ showing the location of the quasar and nucleus apertures.

nuclear point source can be found in table 2.5. A strong X-ray source is coincident with one of the spiral arms of the galaxy where several knots of emission can be seen in the optical image (see figure 2.19). The remnant of the historic Type II supernova SN 1941C seen in NGC 4136 is not located close to this X-ray source (Van Dyk, Hamuy \& Filippenko 1996).

NGC 4144: This galaxy was observed for the first time in X-rays and no emission was detected in the ROSAT HRI data. A $2 \sigma$ upper limit for a point source located in the nuclear region of the galaxy can be found in table 2.5 .

NGC 4150: A strong point-like source coincident with this galaxy was detected in the ROSAT All-Sky Survey with $F_{X}=6 \times 10^{-13} \mathrm{ergs} \mathrm{s}^{-1} \mathrm{~cm}^{-2}$ and a photon index $\Gamma=1.41$. The emission was assumed to be from the nucleus of NGC 4150 (Moran, Halpern \& Helfand 1996; Boller et al. 1998). The high resolution image seen in figure 2.21 shows, however, that the X-ray source is more than $15^{\prime \prime}$ away from the galactic nucleus and has a position consistent with a knot of optical emission. Spectroscopy of the optical counterpart shows that the source is a background quasar at redshift 0.52 (see chapter 3 ).

Figure 2.21 shows that the X-ray contours of the source are elongated in the north west direction, suggesting that some emission might be coming from the nuclear region of the galaxy. An estimate of the nuclear emission was obtained using a $10^{\prime \prime}$ radius aperture 


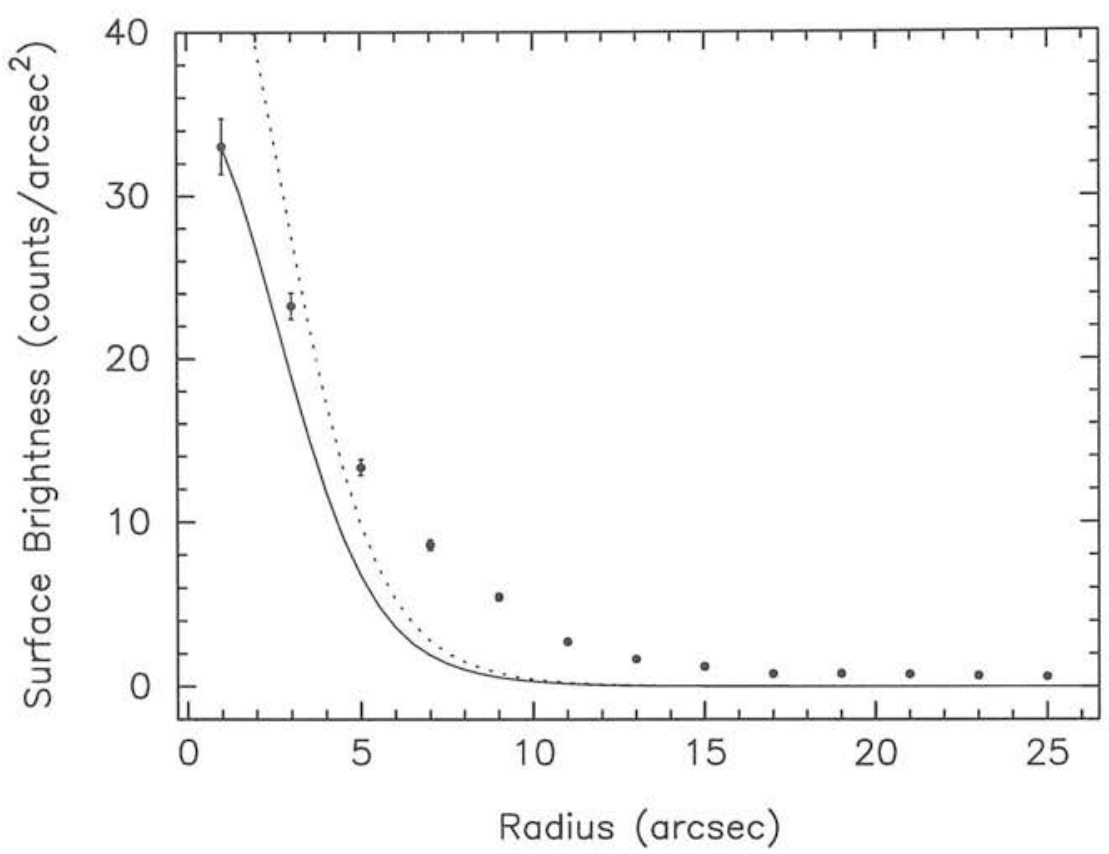

Figure 2.37: Observed profile and model PSF for the nuclear X-ray source in NGC4736. Models as in figure 2.34 .

located as shown in figure 2.36. Although the observed counts have a $\mathrm{S} / \mathrm{R} \sim 2.6$ (and so would be considered a significant detection by the criteria defined in section 2.2.3) the measurement will be treated as an upper limit because of contamination from the nearby quasar.

NGC 4236: This galaxy has a very low surface brightness and no obvious nucleus (Johnson 1997). From the ROSAT HRI observations reported here no X-ray sources have been found in the central region of the galaxy. The only detected source $(\mathrm{X}-1)$ is located in the galactic plane and might have a faint optical counterpart. A $2 \sigma$ upper limit for a point source located in the nuclear region of the galaxy can be found in table 2.5 .

NGC 4244: No emission was detected in the ROSAT HRI observations of this galaxy. A $2 \sigma$ upper limit for a point source located in the nuclear region of the galaxy can be found in table 2.5 .

NGC 4395: This galaxy contains the faintest and nearest Seyfert 1 nucleus known today. Its weak nuclear $\mathrm{X}$-ray emission is highly variable (see chapter 4 ). The bright source seen in figure $2.24(\mathrm{X} 2)$ has no obvious optical counterpart.

NGC 4605: This galaxy was observed for the first time in X-rays and no emission was detected in the ROSAT HRI data. A $2 \sigma$ upper limit for a point source located in the 
NGC 4826

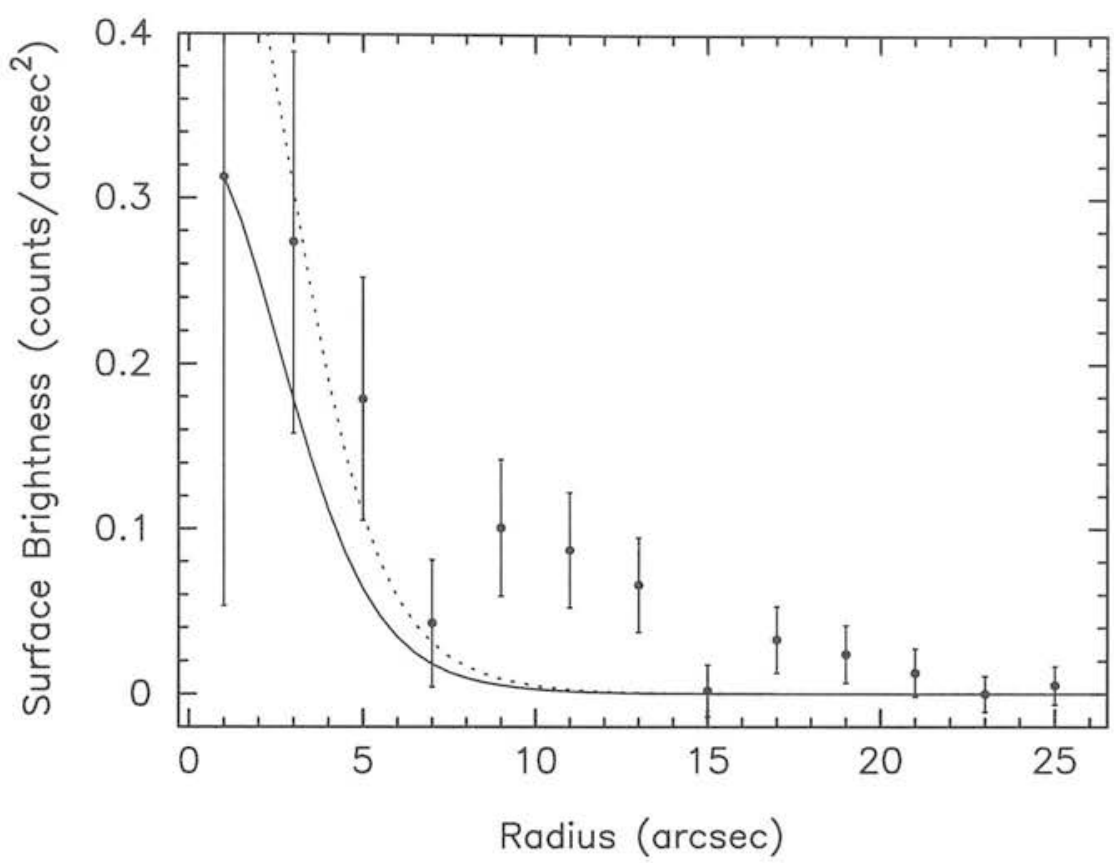

Figure 2.38: Observed profile and model PSF for the nuclear X-ray source in NGC 4826 . Models as in figure 2.34 .

nuclear region of the galaxy can be found in table 2.5 .

NGC 4736: Strong X-ray emission is associated with the LINER nucleus of this galaxy. A nuclear point source and significant extended emission can be seen in figure 2.26. The azimuthally averaged profile of this source and the HRI model PSF are shown in figure 2.37 .

The galaxy was previously imaged with the Einstein HRI. A nuclear flux of $2.0 \times 10^{-12}$ ergs $\mathrm{s}^{-1} \mathrm{~cm}^{-2}$ was measured within an aperture of $60^{\prime \prime}$ radius in the $0.5-4.0 \mathrm{keV}$ bandpass (Fabbiano, Kim \& Trinchieri 1992). The fluxes measured from the ROSAT HRI observation using a small $\left(\mathrm{r}=10^{\prime \prime}\right)$ and a large aperture $\left(\mathrm{r}=100^{\prime \prime}\right)$ are $1.4 \times 10^{-12}$ and $4.7 \times 10^{-12} \mathrm{ergs} \mathrm{s}^{-1} \mathrm{~cm}^{-2}$ respectively (see table 2.4 ). The change in the total observed flux can be explained if the central source is variable or if a substantial fraction of its emission is radiated in the very soft $\mathrm{X}$-rays $(k T \lesssim 0.5 \mathrm{keV})$.

Roberts, Warwick \& Ohashi (1999) have recently reported on ASCA and ROSAT PSPC and HRI observations for NGC 4736. They find that the nuclear emission is consistent with an unresolved source plus an extended component. PSF modelling shows that the unresolved source accounts for more than $50 \%$ of the detected emission (Roberts, Warwick \& Ohashi 1999). The 0.1-10 keV ASCA spectrum of the emission is consistent with a power-law with index $\alpha \sim 1$ plus a softer thermal component $(k T \sim 0.1-0.6 \mathrm{keV})$ which 


\begin{tabular}{|c|c|c|}
\hline Source & $\begin{array}{c}\text { CR }(20 / 01 / 93) \\
\left(\text { counts s }{ }^{-1}\right)\end{array}$ & $\begin{array}{c}\text { CR }(30 / 07 / 94) \\
\left.(\text { counts s s })^{-1}\right)\end{array}$ \\
\hline $\mathrm{X}-1$ & $1.27 \pm 0.31$ & $0.38 \pm 0.22$ \\
\hline$X-2$ & $1.18 \pm 0.31$ & $1.39 \pm 0.32$ \\
\hline $\mathrm{X}-3$ & $15.20 \pm 0.86$ & $17.57 \pm 0.91$ \\
\hline$X-4$ & $0.75 \pm 0.27$ & $0.88 \pm 0.28$ \\
\hline $\mathrm{X}-5$ & $0.67 \pm 0.26$ & $0.64 \pm 0.25$ \\
\hline$X-6$ & $2.08 \pm 0.37$ & $2.43 \pm 0.39$ \\
\hline$X-7$ & $1.91 \pm 0.36$ & $0.05 \pm 0.19$ \\
\hline $\mathrm{X}-8$ & $1.31 \pm 0.32$ & $0.17 \pm 0.20$ \\
\hline
\end{tabular}

Table 2.7: Count rates (in units of $10^{3} \mathrm{~s}^{-1}$ ) for the point sources observed in NGC 5236 from two ROSAT HRI observations obtained in January 1993 and July 1994.

dominates below $2 \mathrm{keV}$.

NGC 4826: The nucleus of NGC 4826 has been classified as a transition object (a combination of a LINER and an HIIR nucleus) by Ho, Filippenko \& Sargent (1995b). The galaxy was observed with the Einstein IPC and a nuclear flux of $7.89 \times 10^{-13} \mathrm{ergs} \mathrm{s}^{-1} \mathrm{~cm}^{-2}$ was measured within a $4.5^{\prime}$ radius aperture (Fabbiano, Kim \& Trinchieri 1992), in good agreement with the flux found in table 2.4. Figure 2.38 shows the azimuthally averaged profile as observed by the ROSAT HRI. Significant extended emission is observed within $\gtrsim 20^{\prime \prime}$ of the central peak.

NGC 5204: Einstein IPC observations of this galaxy show strong X-ray emission with a total flux of $9.6 \times 10^{-13}$ ergs s${ }^{-1} \mathrm{~cm}^{-2}$ (Fabbiano, Kim \& Trinchieri 1992) in good agreement with the measurement given here (see table 2.4). Population I XRBs were thought to be responsible for this emission since the number of $O B$ stars inferred from IUE observations were in agreement with the X-ray luminosity (Fabbiano \& Panagia 1983). However, the HRI image (see figure 2.28) shows that the X-ray emission is consistent with a single off-nuclear point source $\sim 17^{\prime \prime}$ away from the nucleus. This position is confirmed by two different HRI observations obtained 6 months apart.

Three SNRs have been identified in NGC 5204, but none of them is coincident with the position of the source (Matonick \& Fesen 1997). Although there is no obvious optical counterpart for the $\mathrm{X}$-ray source, several nearby optical knots can be seen in figure 2.28 . The spectrum of one of the candidates is presented in chapter 3 and corresponds to a star forming region. The extremely high luminosity of this source, together with the lack of variability observed between the Einstein and the ROSAT observations, favour a SNR from a class of super-luminous remnants (Schlegel 1994b) (the SASS report does not find conclusive evidence of variability during the HRI observations, either). 


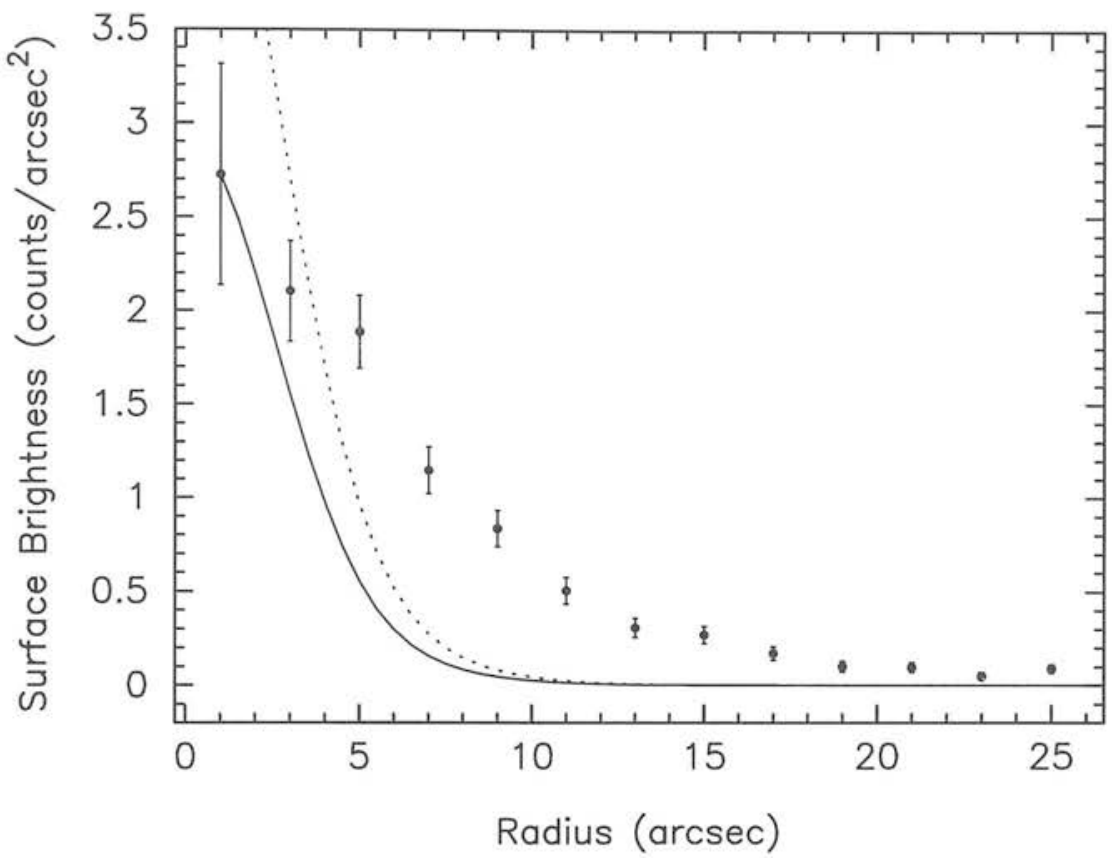

Figure 2.39: Observed profile and model PSF for the nuclear X-ray source in NGC 5236 . Models as in figure 2.34 .

NGC 5236: The very complex X-ray emission in this galaxy can be appreciated in figure 2.29. Several X-ray knots are distributed on top of bright, uneven extended emission. Comparison between the two ROSAT HRI observations reveals that at least three of the eight detected point sources are variable. Table 2.7 shows the count rates observed in January 1993 and July 1994. Sources X-1, X-7 and X-8 are not detected during the observations obtained in July 1994, but are among the brightest objects seen in January 1993.

Trinchieri, Fabbiano \& Paulumbo (1985) reported on the Einstein HRI observations of this galaxy. From their observations (obtained in January 1980 and February 1981) only 3 sources were detected in the nuclear region of the galaxy. They correspond to the ROSAT HRI sources X-2, X-3 (the nucleus) and X-6, which are the brightest sources observed in the ROSAT HRI data acquired in July 1994 (see table 2.7). Their fluxes in the Einstein (0.5-3.0 keV) band-pass are in good agreement with the fluxes given in table 2.4

Ehle et al. (1998) report on ROSAT PSPC observations of NGC 5236 obtained between January 1992 and January 1993. They find a luminosity for the nuclear source of $7 \times 10^{-13}$ ergs s${ }^{-1} \mathrm{~cm}^{-2}$, in good agreement with the measurement in table 2.4. They also detect all the point sources seen in the HRI observations, with the exception of X-5. This suggests that the variable sources $\mathrm{X}-1, \mathrm{X}-7$ and $\mathrm{X}-8$ were detectable for at least a year (from the beginning of 1992 until the beginning of 1993), before fading away, becoming undetectable 


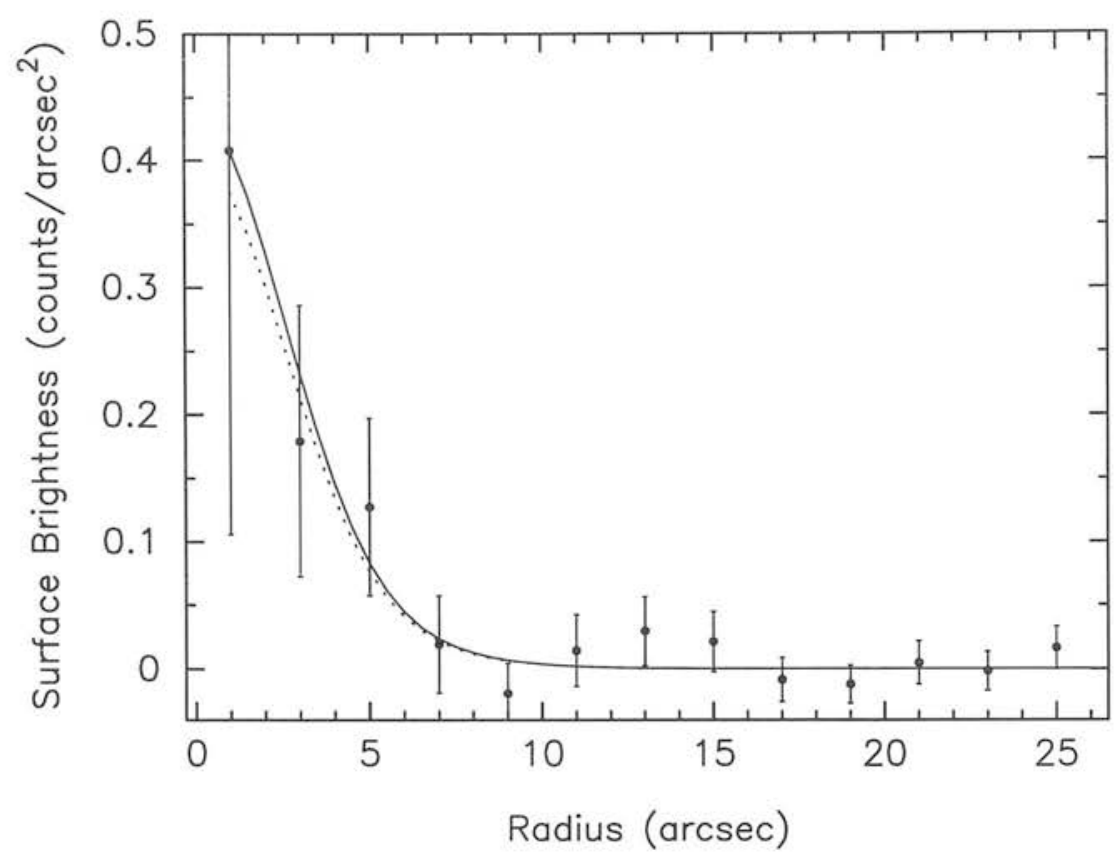

Figure 2.40: Observed profile and model PSF for the nuclear X-ray source in NGC 5457 . Models as in figure 2.34 .

by July 1994 .

The diffuse emission from the central region of NGC 5236 can be appreciated in figure 2.39. From the PSPC observations Ehle et al. (1998) find that the soft 0.1-0.4 keV diffuse component accounts for almost half of the total X-ray emission and argue that most of it is due to hot gas in a super bubble with radius $\sim 10-15 \mathrm{kpc}$. Evidence of vigorous starburst activity comes from observations of the nuclear and circumnuclear regions of NGC 5236 which have intricate morphologies in the UV, optical and infrared (Bohlin et al. 1983; Johnson 1997; Gallais et al. 1991).

Finally, several historic supernovae have been observed in this galaxy, but none of them is consistent with the positions of the point X-ray sources.

NGC 5238: This galaxy was observed for the first time in X-rays and no emission was detected in the ROSAT HRI data. A $2 \sigma$ upper limit for a point source located in the nuclear region of the galaxy can be found in table 2.5 .

NGC 5457: Observations of this galaxy with the Einstein IPC and ROSAT PSPC have been widely reported (Trinchieri, Fabbiano \& Romaine 1990; Williams \& Chu 1995; Snowden \& Pietsch 1995). The shortest of the two ROSAT HRI images reported here was briefly discussed by Williams \& Chu (1995). Several strong point sources are thought to be asso- 


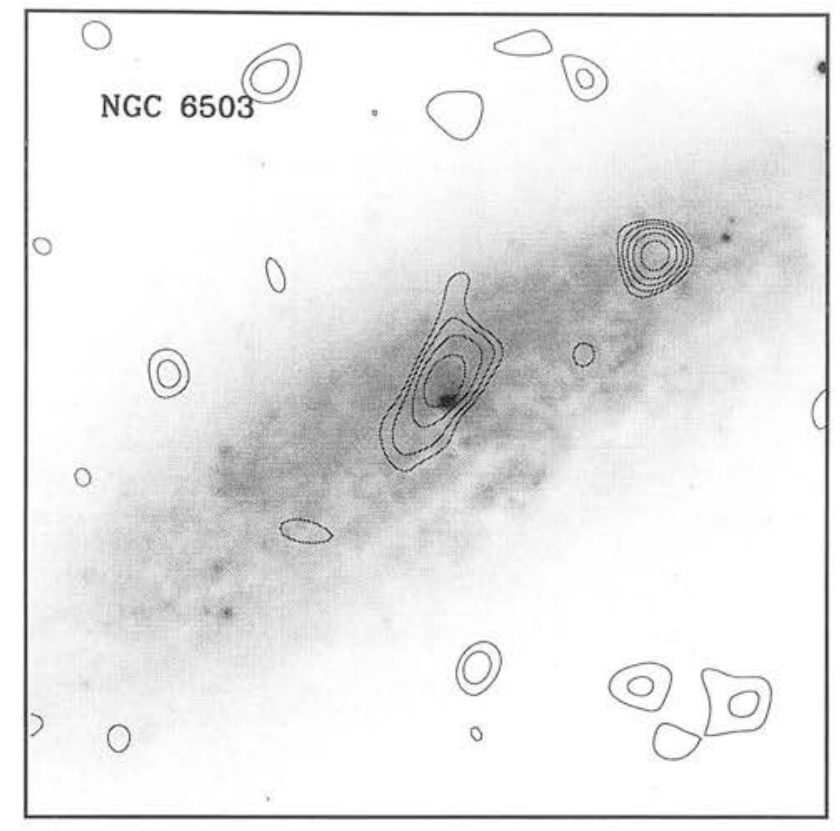

Figure 2.41: X-ray isocontours plotted on top of an optical JKT image of NGC 6503 . The X-ray image was produced binning the raw frame into $2^{\prime \prime}$ pixels and smoothed using Gaussians with $\sigma=4^{\prime \prime}$. Contours were drawn at $1.5^{n}$ times the standard deviation per pixel in the smoothed background, where $n=1,2,3$, etc.

ciated with giant HIIRs (Williams \& Chu 1995), but they are not visible in the $\sim 6^{\prime} \times 6^{\prime}$ optical JKT image.

From IPC data Trinchieri, Fabbiano \& Romaine (1990) find a nuclear X-ray flux of $3 \times$ $10^{-13} \mathrm{ergs} \mathrm{s}^{-1} \mathrm{~cm}^{-2}$ within a circle of $90^{\prime \prime}$ radius for the Einstein $(0.2-4.0 \mathrm{keV})$ band-pass. From the ROSAT HRI observations a nuclear flux of $4 \times 10^{-14} \mathrm{ergs} \mathrm{s}^{-1} \mathrm{~cm}^{-2}$ is obtained, an order of magnitude fainter than the IPC flux. The difference can be explained if sources $\mathrm{X}-2$ and $\mathrm{X}-3$, which are not resolved by the IPC, were contained within the large aperture used by Trinchieri, Fabbiano \& Romaine (1990), or by the effect of luminous and highly variable XRBs.

The presence of a soft diffuse component is discussed by Snowden \& Pietsch (1995). They find conclusive evidence in ROSAT PSPC observations for extended emission within the inner $7^{\prime}$ of the galaxy. The radial profile obtained from the ROSAT HRI observations shown in figure 2.40 suggests some patchiness in the X-ray emission but no firm evidence for a central extended component is found.

An astonishing total of 93 SNRs have been identified in the galaxy (Matonick \& Fesen 1997). Remnants 57 and 54 are consistent with the positions of sources X-2 and X-3 seen in figure 2.31 . 

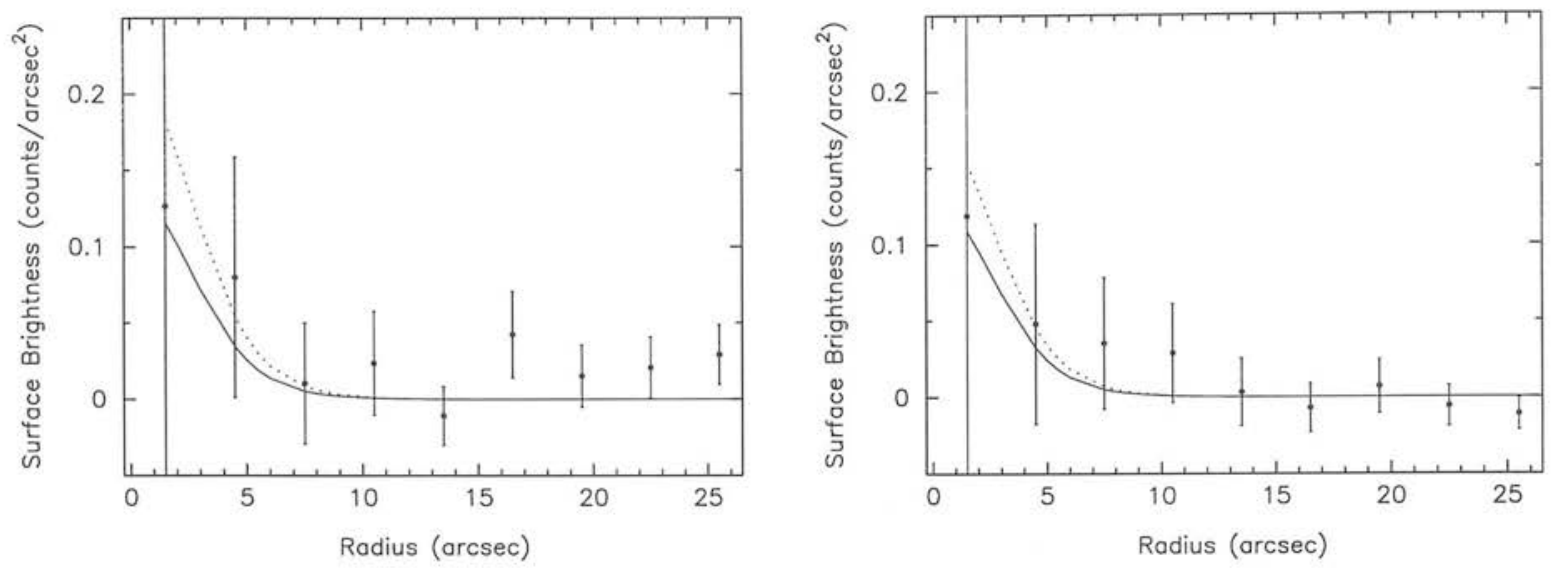

Figure 2.42: Observed profile and model PSF for the nuclear X-ray source in NGC6503. Region 1 corresponds to the profile obtained along to the direction of the extended emission, while region 2 was obtained in the perpendicular direction. Models as in figure 2.34 .

NGC 6503: The nucleus of this galaxy has been classified as a combination of a transition object and a Seyfert 2 nucleus by Ho, Filippenko \& Sargent (1995b). The ROSAT HRI observations in figure 2.32 show extended and elongated emission in the central region of the galaxy. This diffuse emission can be better appreciated in figure 2.41 where the $\mathrm{X}$-ray image has been smoothed using a Gaussians with $\sigma=2^{\prime \prime}$. Figure 2.42 shows radial profiles of the source obtained by averaging pie-slice shaped regions aligned along and perpendicular to the direction of the extended emission. Although the signal to noise ratio is low, more clumpiness is observed in the profile obtained along the extended emission.

The extended source lies $\sim 10^{\prime \prime}$ away from the galactic nucleus. Although shifts of $\sim 5^{\prime \prime}$ are normal during pointed ROSAT observations (Fabbiano \& Juda 1997; Vogler \& Pietsch 1996) the observed extended X-ray emission is likely to be off-nuclear because a bright source located $\sim 5.6^{\prime}$ away from the galaxy and identified as QSO $1749+701$ is consistent with an optical counterpart found in a DSS plate (note however, that the astrometric solution of the JKT optical image and the DSS plate may have systematics shifts of a few arc-seconds - see chapter 3 ).

NGC 6946: Six historic SNs have been seen in this spiral galaxy (SNs 1917A, 1939C, 1948B, 1968D, 1969P, and 1980K) (Barbon, Capellaro \& Turatto 1989). A total of 27 remnants (not including the historic SNs) have been detected by Matonick \& Fesen (1997). SN 1980K has been observed in X-rays (Schlegel 1994a), but the source lies outside the $\sim 6^{\prime} \times 6^{\prime}$ JKT optical image of the galaxy. Another remnant in this galaxy belongs to an extreme group of objects with X-ray luminosities $z 10^{39} \mathrm{ergs} \mathrm{s}^{-1}$ (Schlegel 1994b). It corresponds to source $\mathrm{X}-8$ in figure 2.33 . 

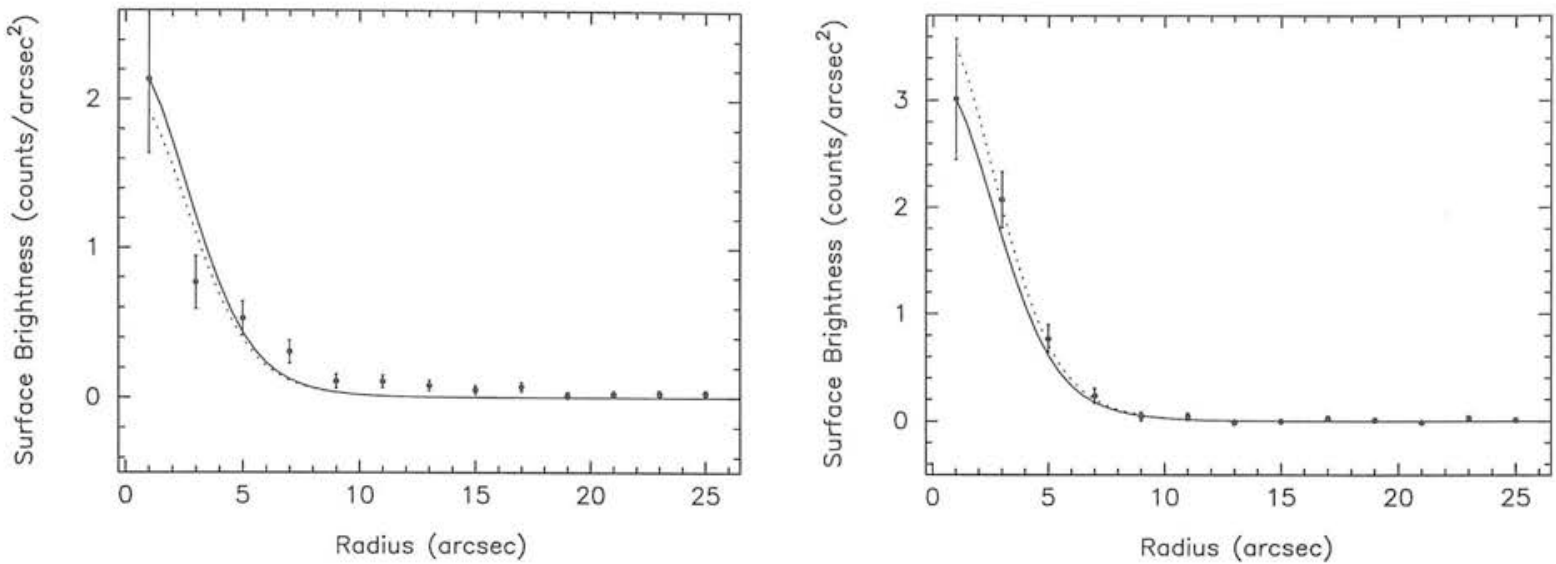

Figure 2.43: Observed profile and model PSF for the nuclear X-3 source (left) and the off-nuclear X-7 source (right) in NGC 6946. Models as in figure 2.34.

Recent HST images show that the source corresponds to several interacting SNRs (Blair, Fesen \& Schlegel 1997). Schlegel (1994c) reports the presence of diffuse emission across the disk of the galaxy from ROSAT PSPC observations. Figure 2.43 shows the profile of the nuclear source $\mathrm{X}-3$ and of an off-nuclear source $(\mathrm{X}-7)$. Comparing both plots it is clear that the nuclear source is extended up to $\sim 20^{\prime \prime}$ away from the central peak. 


\section{Chapter 3}

\section{Optical spectroscopic observations}

\subsection{Selection of targets and astrometry}

From the broad band near-IR, radio, optical, and X-ray images, targets in the nuclear regions of the galaxies were selected for long slit spectroscopy with the William Herschel Telescope (WHT). While some galaxies had a distinctive nucleus, detected at several different wavelengths, others had multiple hot spots, possibly appearing in different wavelength bands, with no single, well defined, unique nucleus.

At the WHT spectroscopic targets are usually acquired manually. Once the object is seen in the TV monitor, the pointing is gradually corrected until the object moves into the slit.

However, many of the targets had either very faint or no optical counterparts on the TV display ( $V \gtrsim 20$ magnitudes). For the acquisition of these objects it was necessary to obtain accurate positions for the targets relative to a brighter reference source so that the observations could be done in 'blind offset' mode. This procedure can be achieved with a precision of $<0.2^{\prime \prime}$ (RMS) for offsets over $z 20^{\prime}$ on the sky.

To calculate the offset requires either coordinates of both objects in a common reference frame, or accurate absolute coordinates from separate sources. The offsets were calculated as described in the following sections.

\subsubsection{Optical sources in JKT images}

The astrometry for the JKT images was made using celestial coordinates from charts generated by the Automatic Plate Measuring machine (APM) at the Royal Greenwich 


\begin{tabular}{|llll|}
\hline \hline $13 / 06 / 96$ & $14 / 06 / 96$ & $15 / 06 / 96$ & $15 / 01 / 97$ \\
\hline Maffei I & Maffei II & Maffei I & IC 342 \\
NGC 147 & NGC 205 & NGC 185 & Leo B \\
NGC 404 & NGC 221 & NGC 224 & NGC 1560 \\
NGC 4395 & NGC 598 & NGC 247 & NGC 2366 \\
NGC 4736 & NGC 4826 & NGC 4605 & NGC 2403 \\
NGC 5457 & NGC 5204 & NGC 5204 & NGC 2976 \\
NGC 6503 & NGC 6946 & NGC 5238 & NGC 3738 \\
& & & NGC 4144 \\
& & & NGC 4150 \\
& & & NGC 4236 \\
& & & UGC 6456 \\
& & & UGC 7321 \\
\hline BD+284211 & BD+28 4211 & BD +332642 & FEIGE 34 \\
G158-100 & BD+332642 & HD 192281 & G191-B2B \\
PG 1708+602 & HD 192281 & PG 1708+602 & \\
\hline \hline
\end{tabular}

Table 3.1: Journal of spectroscopic observations. Standard stars are also included.

Observatory in Cambridge. The main sources of material for APM are the UK Schmidt Telescope, Palomar Schmidt and Curtis Schmidt photographic plates.

For each of the JKT images several (normally 5 to 10 ) stars with coordinates in the APM charts were identified and their pixel positions in the JKT images determined using a centroid algorithm. The astrometric solutions were found using the STARLINK package ASTROM, which solves a six coefficient linear model for each image (zero points, scales in x and $y$, orientation and non-perpendicularity), using the pixel positions and APM celestial coordinates of the reference stars. Once the astrometry has been determined, relative positions in the APM frame of reference can be found for the faint targets. Several tests were carried out which showed that the coordinates of the reference sources could be recovered with an accuracy $\sim 0.1^{\prime \prime}$.

\subsubsection{Optical Sources without JKT Images}

For galaxies without optical JKT images, plates from the Digitised Sky Survey (DSS) were used instead. DSS images already have astrometric solutions in their headers as a set of 'plate solution coefficients'. Comparing the DSS astrometry with the APM solutions it was found that relative positions agreed with an accuracy of $\sim 0.1^{\prime \prime}-0.2^{\prime \prime}$. However, absolute positions showed systematic shifts of a few arc seconds, depending on the particular DSS plate and APM chart used. 
For a few galaxies with JKT images, but without available APM charts, the astrometric solution was found using ASTROM as described earlier, but using celestial coordinates from a DSS plate. In this case the astrometric solution is tied to the DSS frame of reference, which as has been mentioned, can have a systematic shift from the APM solution.

With the astrometric solutions determined from APM charts and DSS plates, faint optical sources (or X-ray and radio sources with faint optical counterparts) could be acquired using a blind offset from a reference star with an accuracy of $\sim 0.1^{\prime \prime}-0.2^{\prime \prime}$.

\subsubsection{Radio sources without optical counterparts}

Radio sources without optical identifications present a different problem. VLA positions have an absolute accuracy better than 0.1" (Perley 1997) and so a blind offset from an accurate absolute optical source should be reliable. Unfortunately, however, shifts in the absolute positions of objects in the APM and DSS frames of reference have been detected and it is difficult to test if either is a good absolute system since astrometric stars are normally very bright, appearing as non-point-like (saturated) sources in the two catalogues.

Instead, the 'Catalogue of Positions and Proper Motions' (PPM) was chosen as the source for reference stars to blind offset to the position of radio sources. The average mean errors of the positions in the PPM catalogue is $0.27^{\prime \prime}$ (Roeser \& Bastian 1991).

\subsubsection{X-Ray sources without optical or radio counterparts}

X-ray sources without optical (or radio) counterparts were not followed up since the absolute accuracy in the X-ray positions is $\sim 5^{\prime \prime}$ (see chapter 2 ) and the slit could not be placed on the target without a better knowledge of its location.

\subsection{The WHT and ISIS}

The William Herschel Telescope (WHT) is part of the The Isaac Newton Group of Telescopes (ING) which is located on the island of La Palma as part of the Observatorio del Roque de los Muchachos. The observatory site is operated by the Instituto de Astrofísica de Canarias. The ING is operated on behalf of the UK Particle Physics \& Astronomy 


\begin{tabular}{|c|c|c|c|c|}
\hline & $\begin{array}{l}\text { July } 1996 \\
\text { Blue Arm }\end{array}$ & Red Arm & $\begin{array}{l}\text { January } 1997 \\
\text { Blue Arm }\end{array}$ & Red Arm \\
\hline CCD & LORAL & Tek & Tek & Tek \\
\hline gain ( $\overline{\mathrm{e}} / \mathrm{ADU})$ & 8.0 & 1.1 & 1.1 & 1.2 \\
\hline readout noise $(\overline{\mathrm{e}})$ & 6.0 & 3.6 & 3.6 & 4.4 \\
\hline pixel size $(\mu \mathrm{m})$ & 15 & 24 & 24 & 24 \\
\hline full frame (pix) & $2148 \times 2148$ & $1124 \times 1124$ & $1124 \times 1124$ & $1124 \times 1124$ \\
\hline windowed frame (pix) & $2148 \times 1055$ & $1124 \times 650$ & $1124 \times 650$ & $1124 \times 650$ \\
\hline grating & R300B & R316R & R300B & $\mathrm{R} 316 \mathrm{R}$ \\
\hline wavelength range $(\AA)$ & $3538-5460$ & $5312-6807$ & $3655-5223$ & $5270-6765$ \\
\hline spectral resolution $(\AA)$ & $\sim 3.8$ & $\sim 3.0$ & $\sim 3.5$ & $\sim 3.0$ \\
\hline dispersion $(\AA /$ pix $)$ & 0.96 & 1.47 & 1.54 & 1.47 \\
\hline spatial scale ("/pix) & 0.22 & 0.36 & 0.36 & 0.36 \\
\hline
\end{tabular}

Table 3.2: Set-up for the spectroscopy

Research Council (PPARC) and the Nederlandse Organisatie voor Wetenschappelijk Onderzoek (NWO).

The WHT is an alt-azimuth, 4.2 metre telescope with a focal ratio (focal length / mirror diameter) at the Cassegrain focus of 10.94. The alt-azimuth design of the telescope means that the field of view rotates as the telescope tracks. To compensate for this movement instruments and detectors at the Cassegrain focus must be mounted on turntables.

ISIS is a two armed spectrograph mounted at the Cassegrain focus of the WHT. The upper arm is optimised for the range $3000-6000 \AA$, and is called the BLUE arm, while the lower arm is optimised for the range $5000-10000 \AA$, and is called the RED arm. Light is diverted into the two arms by $45^{\circ}$ folding mirrors or dichroic filters. The use of dichroics allows for the simultaneous use of both ISIS arms.

After encountering the folding mirror or dichroic, the light passes through the collimators to produce a collimated beam of $150 \mathrm{~mm}$ diameter. The light is then reflected by the gratings to the CCD cameras. The spectrograph arms are normally focussed by adjusting the collimator positions.

\subsection{Observations, data reduction and calibration}

\subsubsection{Observing runs}

Long slit spectra of the nuclear targets were obtained on the 13th. 14th and 15th of July 1996 and the 15th of January 1997. Table 3.1 shows the journal of observations. 


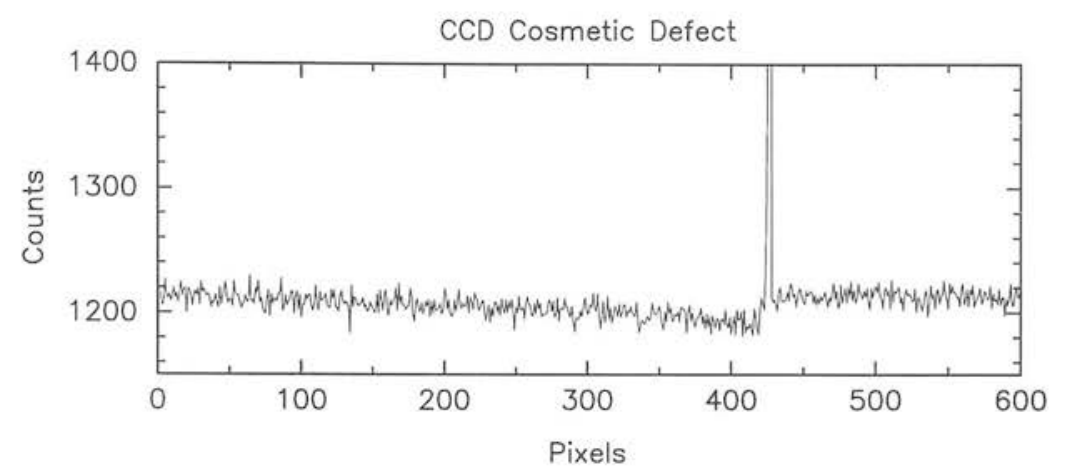

Figure 3.1: Dark tail produced after bright pixels in a raw frame obtained with the Loral CCD.

The R316R grating was installed in the red arm of ISIS and the R300B was used in the blue arm, together with dichroic 5400 (half power point of crossover at $5470 \AA$ ). The wavelength coverage was $\sim 3650$ - $6750 \AA$. A small gap between 5220 and $5270 \AA$ was not observed during January 1997. A TEK CCD and a Loral CCD were used during July 1996 for the red and the blue camera, respectively. Both cameras were equipped with TEK CCDs for the run in January 1997. The slit width was $1^{\prime \prime}$ on the sky, resulting in a spectral resolution of $\sim 3.0 \AA \mathrm{FWHM}$ for the red arm and $\sim 3.5 \AA \mathrm{FWHM}$ for the blue arm $(\sim 3.8$ $\AA$ FWHM with the Loral chip) as measured from bright unblended lines in comparison lamp frames.

The standard stars were acquired using a slit width of $8^{\prime \prime}$. The seeing varied between $\sim 0.8$ to $\sim 1.2$ arcsecs during both runs. The dispersion achieved in the red arm was $1.47 \AA$ per pixel (0.062 $\AA$ per micron). In the blue arm the dispersion was $0.96 \AA$ per pixel with the Loral and $1.54 \mathrm{~A}$ per pixel with the Tek detector $(0.064 \AA$ per micron). In both runs the slit was positioned at the parallactic angle to minimize light losses. The CCDs were windowed to reduce the read-out times and covered $4^{\prime}$ in the spatial direction with a scale of $14.9^{\prime \prime} / \mathrm{mm}$. A summary of the set-ups can be seen in table 3.2.

Apart from the normal cosmetic defects found in all CCDs (e.g., hot pixels, charge-trap columns), the LORAL CCD showed a dark tail after bright pixels, as can be seen in figure 3.1. The problem is only apparent when pixels have $z 2000$ counts in the raw frame. The depth of the depression seems to be correlated with the peak count. The data shown in figure 3.1 were produced with a large cosmic ray (49447 peak counts) and the depression extends over up to 300 pixels $(\sim 270 \AA)$.

During the run of July 1996 no clouds were present, although the presence of Saharan 


\begin{tabular}{|ccccc|}
\hline \hline Date & UT & Extinction (V mag) & Variation (mag/hr) & N \\
\hline Jul 13/14 & $01: 20$ & $0.13 \pm 0.012$ & - & 46 \\
Jul 14/15 & $01: 19$ & $0.74 \pm 0.026$ & $0.017 \pm 0.004$ & 38 \\
Jul 15/16 & $01: 19$ & $0.46 \pm 0.012$ & $-0.021 \pm 0.002$ & 48 \\
\hline \hline
\end{tabular}

Table 3.3: Extinction during the run in July 1996

dust in the atmosphere hampered the observations. Table 3.3 summarizes the extinction conditions measured on site by the Carlsberg Meridian Circle. The extinction values are for the given UT time. The number of observations used to determine the extinction values is also given. During the 13th of July the dust produced a constant extinction, while during the 14 th and 15 th conditions gradually changed during the nights.

The conditions during January 1997 were not photometric throughout the night as clouds covered the sky. However, towards the end of the night some data and associated standard stars were acquired during clear periods. No extinction information from the Carlsberg Meridian Circle was available due to the poor weather conditions.

\subsubsection{Data reduction}

The data were reduced using IRAF software. The first step was the bias correction. The bias is a platform level added to the output signal in order to make sure that the Analogueto-Digital Converter (ADC) always receives a positive value. The ADC is responsible for converting the amount of charge (or number of electrons) produced by the incidence of photons on a CCD pixel to the measured value. The bias has to be determined for each frame as it might vary slightly with telescope position, temperature, etc, and might also vary as a function of the position on the CCD. In order to find the bias level there are two tools that can be used: the bias frames and the overscan region. The bias frames are images which are produced by reading out the CCD without exposure. The overscan region is a $\sim 30$ pixel strip right at the edge of each image that is not exposed. By combining several bias frames it is possible to look for structures in the bias, while the overscan region allows for a better determination of the bias level in each individual image.

Inspection of the bias frames produced with the LORAL CCD showed a strong 'ramp' on one side of the chip, probably produced by the system electronics (see figure 3.2 ). The ramp varied in height and width from image to image, but presented a constant profile across a single frame and always affected images with short exposures. A correlation was found where images with the shortest exposure times presented the biggest ramps, and therefore bias frames were badly affected. The idea of modelling the ramp in order to 


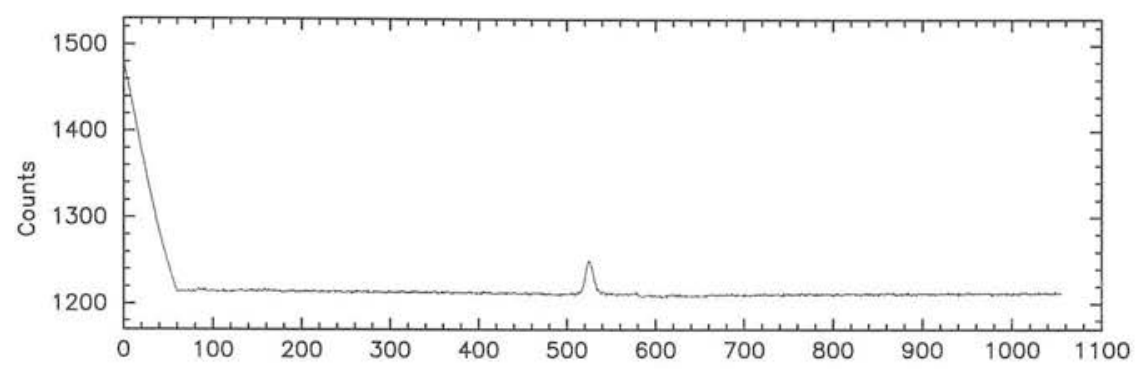

Figure 3.2: Overscan region of a frame obtained with the LORAL CCD. It is possible to see the 'ramp' on the left of the profile as well as an emission feature in the center produced by the bleeding of charges.

remove it from the images was discarded as closer examination showed complex sinusoidal structures in the profile. It was finally decided that no bias frames were going to be used for the reduction of data obtained with the LORAL CCD. Information about the bias level was inferred exclusively from the overscan regions. Note that the ramp was normally small in the long exposed frames of program objects and that the region of the interest (the central part of the chip) was not affected whatever the ramp size.

All images obtained with Tek CCDs were corrected by the bias frame to remove the structures observed in the bias level from the data. Since the number of bias frames obtained during the 14th and 15th of July 1996 were low (see table 3.4), the combined bias frame from the first night of the run was always used. Profiles in the spatial and dispersion axis of the combined bias frame for the 13th of July 1996 and the 15th of January 1997 are shown in figure 3.3. It can be seen that the bias level indeed shows spatial structures.

Figure 3.2 shows the overscan region of a bright standard star frame obtained with the LORAL chip. The profile illustrates another problem that affected both arms during July 1996: structures in the overscan strips due to bleeding of charges from the exposed regions of the CCDs (in this case an emission structure at the position of the standard star on the CCD). Consequently, to estimate the bias level for the images, a straight line was fitted to the collapsed overscan region, but the pixels containing the structures due to the bleeding of charges were excluded. The fitted level was then subtracted from the whole image.

After correcting for the bias level, the images were flat-fielded. This step removes the pixel to pixel variation in sensitivity of the CCD, so that if the detector is illuminated directly by a completely uniform beam of radiation the output signal would be, within the noise, the same at all points on the CCD. Apart from the local pixel to pixel changes in the 

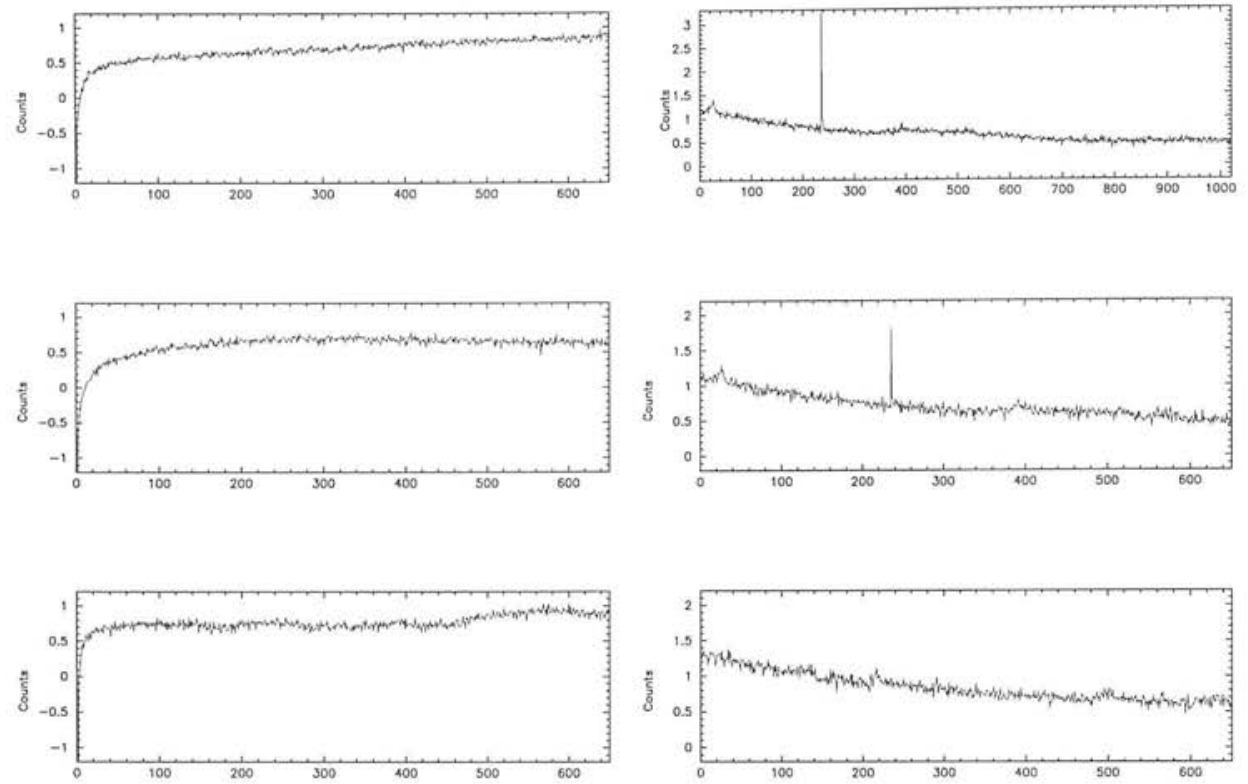

Figure 3.3: Collapsed profiles of bias frames. From top to bottom the bias for the red arm during the 13th of July 1996, and blue and red arms for the 15th of January 1997 are shown. Right panels show profiles in the spatial direction while left panels show profiles in the dispersion direction.

CCD sensitivity, other larger scale gradients in the data can be caused by instrumental vignetting. These patterns are also corrected through flat-fielding.

The starting point of a flat-field correction is the observation of something which should have a uniform brightness. In spectroscopic observations, however, pixels along the dispersion direction are illuminated by photons of increasingly higher energies. Since all emitting sources have an intrinsic spectral shape and the CCD response is a function of the energy of the incident photons, this change in brightness along the dispersion direction must be removed in order to obtain a really flat frame across the whole detector. This is normally achieved using a lamp, whose spectrum presents a smooth variation with wavelength and therefore can be easily modeled and eliminated. (The calibration of the CCD response as a function of photon energy is not part of the flat-fielding process, but of the flux calibration of the frames, see next section - flat-fielding is exclusively a spatial correction). Once lamp frames have been obtained and corrected for wavelength dependent fluctuations, the correction to the observation of program objects is applied by dividing the data by the flat-field.

During the runs several flat-fields for each night (see table 3.4) were first combined in order to improve the signal to noise ratio. The resulting frame was then smoothed using a median filtering in order to keep only the wavelength dependent variations. The median 
filter consisted of a sliding rectangular window whose dimensions were chosen so that pixel to pixel variations were smoothed out without removing the spectral variations of the lamp. Dividing the original flat-field by this smoothed version gives the final flat-field, containing only the spatial variations.

Unfortunately, residuals can appear as a result of flat-fielding the data with a source image (the lamp) that does not illuminate the slit as celestial objects do. This variation can also be a function of wavelength. In order to determine a slit illumination correction sky frames were obtained by observing the evening twilight sky. The sky is a spatially uniform source and its light follows the same optical path to the detector as the radiation from the program objects. However, the sky also presents strong emission lines as a function of wavelength. A first order approach to find the illumination correction is to collapse the whole sky frame in order to eliminate the sky spectral features and obtain a one dimensional profile with the desired correction. However this would remove any possible variations of the profile with wavelength (that were indeed found in the data). In order to take into account this variations four steps were followed: first, the sky frames obtained each night were combined to reduce noise; second, a narrow strip containing the central pixels of the frame along the dispersion axis was expanded to a full image; third, the sky frame was divided by this expanded image in order to normalise its spectral features; and fourth, the frame was slightly smoothed in order to obtain an even map. Finally, all the images were divided by this slit illumination map.

\subsubsection{Spectrum extraction and calibration}

The steps involved in the extraction of the galactic spectra were as follows:

- A cut along the spatial axis of the image was used to find the spectrum.

- An extraction window of width 6 pixels $\left(2.16^{\prime \prime}\right)$ in the Tek CCDs and 10 pixels in the LORAL $\left(2.20^{\prime \prime}\right)$ was defined. Two background windows were defined to either side of the spectrum and as far as possible from it in order to minimize contamination from the galaxy. Special care was taken to avoid the ramp seen on LORAL frames. The average width of the background windows was $\sim 30^{\prime \prime}$.

- The centre of the spatial profile of the spectra was traced as a function of the dispersion axis and a polynomial was fitted with the RMS varying from $\sim 0.01$ pixels for well exposed objects to $\sim 0.5$ pixels for extremely faint objects. 


\begin{tabular}{|l|ll|ll|ll|ll|}
\hline \hline \multirow{3}{*}{ Bias frames } & \multicolumn{2}{|c|}{$13 / 06 / 96$} & \multicolumn{2}{|c|}{$14 / 06 / 96$} & \multicolumn{2}{|c|}{$15 / 06 / 96$} & \multicolumn{2}{|c|}{$15 / 01 / 97$} \\
Flats (lamps) & Blue & Red & Blue & Red & Blue & Red & Blue & Red \\
\cline { 2 - 9 } Sky frames & 12 & 12 & 1 & 1 & 9 & 5 & 15 & 15 \\
Arcs & 3 & 4 & 0 & 4 & 5 & 5 & 15 & 15 \\
\hline \hline
\end{tabular}

Table 3.4: Journal of calibration frames

- At each pixel along the dispersion axis the data within the extraction window (centered spatially on the value determinated by the trace at that point), was summed and the sky background subtracted. A variance-weighted or 'optimal extraction' algorithm was used (Horne 1986), in which the weights used in computing the sum are inversely proportional to their statistical uncertainty.

The wavelength calibration was performed using comparison copper-neon and copperargon lamps. To each target spectrum the nearest lamp exposure in time was assigned. A spectrum was then extracted using an aperture and trace identical to those used for the target. A fifth order Legendre polynomial was fitted to the strongest non-blended emission-lines of the lamp spectrum in order to determine the dispersion solution. RMS values were $\sim 0.1 \AA$ for the blue arm and $\sim 0.02 \AA$ for the red arm. Using the dispersion solution the target spectrum was transformed to a linear wavelength scale by interpolating to a constant wavelength increment per pixel. The chosen increments were $0.9^{\prime \prime} /$ pixel and $1.4^{\prime \prime} /$ pixel for the blue and red arms for July 1996, and $1.5^{\prime \prime} /$ pixel and $1.4^{\prime \prime} /$ pixel for the blue and red arms for January 1997.

The flux calibration has two aims: to remove the intrinsic CCD variations in sensitivity as a function of wavelength and to obtain the counts to flux conversion factor in order to estimate the intrinsic brightness of the program objects. To do this it is necessary to observe spectrophotometric standards (stars with tabulated fluxes as a function of wavelength) and to know how the atmospheric extinction, at the location of the observatory and at the time of the observing runs, changes as a function of air-mass and wavelength.

The atmospheric extinction has two main components: a temporally variable component that does not change with wavelength (i.e., a 'grey' component), and a temporally steady component which is a function of wavelength. King (1985) has determined a mean curve of the wavelength dependent component for the observatory in La Palma. In principle therefore, the total extinction can be determined by applying a 'grey shift' to this mean curve in order to account for the wavelength independent component. This can be done by shifting the mean curve to match the extinction measured on site nightly by the Carlsberg 
The spectrophotometric standards observed during the July 1996 and January 1997 runs are shown in table 3.1. The tabulated absolute spectral energy distributions for the standards were obtained from Oke (1990) and Massey et al. (1988). The standard star data were binned to match the bandpass widths in the tabulated fluxes. Using the IRAF package SENSFunc, a calibration function $C_{\lambda}$ was determined for each observation of a standard star as follows:

$$
C_{\lambda}=2.5 \times \log \left(\frac{O_{\lambda}}{T B_{\lambda} F_{\lambda}}\right)+A \times E_{\lambda}
$$

where $O_{\lambda}$ is the observed counts in a bandpass of an observation, $T$ is the exposure time, $B_{\lambda}$ is the bandpass width, $F_{\lambda}$ is the flux at the bandpass from the tabulated fluxes of the spectrophotometric standard, $A$ is the airmass, and $E_{\lambda}$ is the extinction as determined by King (1985). Thus, $C_{\lambda}$ is the ratio of the observed count rate per Angstrom corrected by the extinction curve to the expected flux expressed in magnitudes.

Figure 3.4 shows the values $C_{\lambda}$ found for every night in the $5700-6700 \AA$ range. It can be seen that during the 13th of July 1996, when the extinction was constant, the dispersion is smaller than during the 14th and 15th of July 1996, when the extinction was changing at a rate of $\sim 0.02$ magnitudes per hour (see table 3.3). The night of the 15 th of January 1997 shows remarkably little dispersion given the prevailing weather conditions. This suggests very patchy clouds with all the standard star observations acquired during clear periods.

Since the night of the 13th of July 1996 did not show variations in the value of the extinction (see table 3.3), a grey shift correction was applied to the mean extinction curve to match the observed 0.13 magnitudes of extinction at $5500 \AA$. This new function $E_{\lambda}$ was then used as input in equation 3.1. This is possible because the wind-blown Saharan dust in the atmosphere above the Canary Islands is grey to an accuracy $\lesssim 5 \%$ between 0.32 and 1.0 microns (Stickland et al. 1987; Whittet, Bode \& Murdin 1987). Note that this grey correction represents an equal shift to all values $C_{\lambda}$ seen in figure 3.4 for that night, and therefore, it does not change the observed dispersion.

For the 14th and 15 th of July similar grey shifts were first applied using the relationships found in table 3.3 which gives a linear dependence of the extinction with time. The applied corrections however, did not improve the dispersion observed in the standard stars (figure 3.4) and therefore the calibration was ultimately done without applying time dependent 
$13 / 07 / 96$

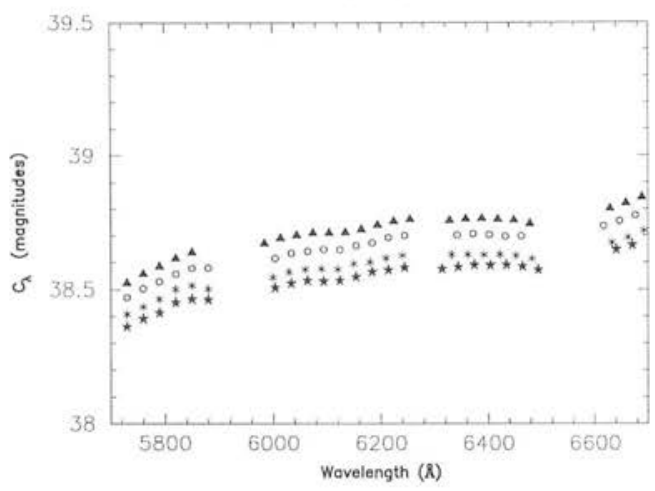

$15 / 07 / 96$

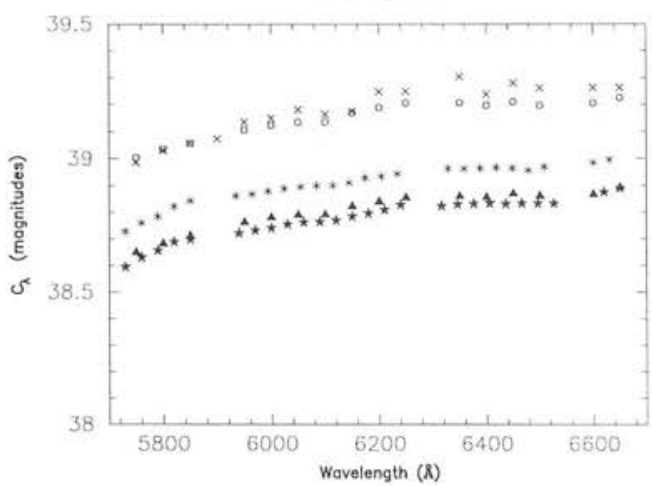

$14 / 07 / 96$

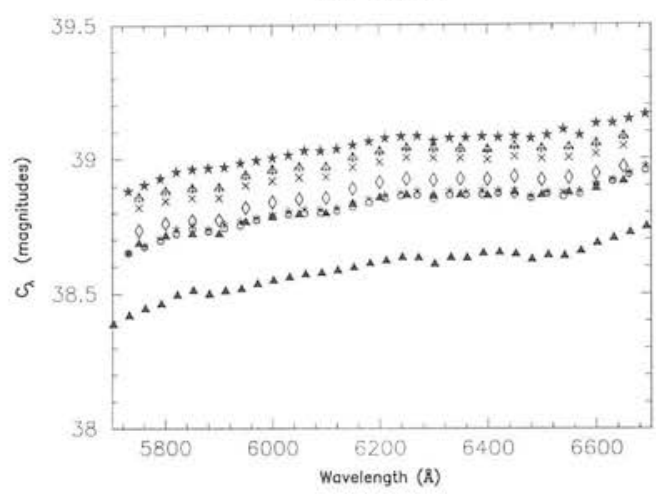

$15 / 01 / 97$

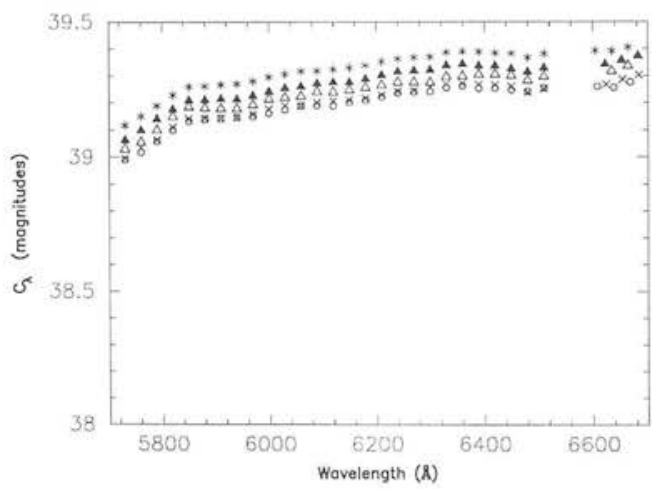

Figure 3.4: Values of $C_{\lambda}$ found using all the standard star observations obtained for every night in the 5700-6700 $\AA$ range (see equation 3.1). For some stars the spectra were not used at wavelengths where strong Balmer absorption lines were located. 
grey shifts to the King mean extinction curve $E_{\lambda}$.

For the 15th of January 1997 the grey flux calibration was done using the standard King extinction curve without any other correction.

A final wavelength dependent calibration function $C_{\lambda}^{F}$ was found for each night by combining all the individual $C_{\lambda}$. This was achieved by applying a grey shift to match the observation of greatest sensitivity. In this way a photometric calibration could be achieved for some objects if their observations, and at least one of the standard stars, were obtained during a clear period. A polynomial was finally fitted to obtain $C_{\lambda}^{F}$ with a typical RMS $\sim 0.01$ magnitudes.

The flux calibration was finally applied to the spectroscopic observations of the targets using the calibration function. Solving equation 3.1 for $F_{\lambda}$ gives:

$$
F_{\lambda}=\left(\frac{O_{\lambda}}{T B_{\lambda}}\right) \times 10^{\left(A \times E_{\lambda}-C_{\lambda}^{F}\right) \times 0.4}
$$

where $O_{\lambda}, T$ and $A$ now correspond to parameters of the observations of the galactic targets.

After obtaining the wavelength and flux calibration for each individual observation, all the available spectra for each target were combined into a final spectrum. If multiple blue (red) observations of a target were available, they were combined into a single blue (red) spectrum. Then the two arms were joined together. The combining of the spectra was done using the IRAF package SCOMBINE. The task averages pixels at common wavelengths after removing deviating values, weighting them by the exposure time.

Finally, badly subtracted sky-lines were removed from the spectra by hand using the IRAF package SPLOT. The spectrum at these wavelengths was interpolated using the nearby continuum. This process introduces uncertainties in the flux values of galactic spectral features located near sky-lines, particularly the Na D $\lambda \lambda 5896,5890$ absorption feature and the $[\mathrm{OI}] \lambda 6300$ emission line. This problem is accentuated when the galaxy has only a small red or blue-shift and/or when the signal to noise ratio of the galactic spectral feature is low. 


\subsection{The spectral atlas}

The atlas consists of the spectra of sources in the nuclear region of the galaxies in the volume-limited sample. The spectra are ordered with increasing right ascension. Although fluxes are given on an absolute scale it must be remembered that most of the observations were not taken under photometric conditions. Those data obtained during the 13th of July 1996 have been labeled with a "(p)" to indicate that they were acquired under photometric conditions despite the presence of the Saharan dust. No correction was made to account for Galactic or internal absorption at this stage (see chapter 5 for Galactic extinction values).

For galaxies with more than one nuclear target, several spectra were acquired. Finding charts for these galaxies are shown in figures 3.5, 3.6 and 3.7. The names given to the spectra in figures 3.8 to 3.28 correspond to the labels in the finding charts. When galaxies had an obvious nucleus with no other candidates for spectroscopy, a single spectrum is shown.

Humps can be seen at the region of overlap between the red and blue spectra in some objects, particularly for observations acquired during the 15th of July 1996. These are due to poor modelling of the rapid decline in sensitivity near the dichroic crossover point, probably due to uncertainties in the wavelength calibration. Since these are the regions used to combine the blue and red spectra, the discrepancies can introduce small relative differences in the continuum level observed in both arms. In figures 3.8 to 3.28 a pair of vertical dotted lines indicate the region of overlap between the blue and red spectra in observations obtained on the 15 th of July 1996.

Wherever corrections have been introduced to the galactic Na D $\lambda \lambda 5896,5890$ feature in order to eliminate badly subtracted sky-lines, this has been indicated in the atlas using small numbers located at the wavelength of the doublet. A number 1 implies a mild correction, a 2 a moderate, and a 3 a strong correction. In the last case the strength of the shown absorption line will deviate significantly from the true value.

Fluxes for selected emission lines are given in table 3.6. The fluxes were obtained using the IRAF package SPLOT. The flux is measured by fitting a Gaussian profile between two continuum points, defined interactively, at either side of the emission line.

To assess the errors associated with the line flux values, Gaussian profile fittings were obtained for lines from spectra with high and low signal-to-noise ratios. The measurements 
were repeated for multiple observations of the same target (i.e., prior to the spectral combination described in the previous section). The measured $\mathrm{H} \alpha$ flux values for high signal-to-noise ratio specta (e.g., NGC 4605 in figure 3.24) showed scatter of less than 1\%, while the flux values for spectra with low high signal-to-noise ratio (e.g., NGC 185 J1 in figure 3.8 ) showed scatter of $10 \%$ to $20 \%$.

Given the good resolution of the observations, the fluxes of Balmer lines were measured directly, without trying to correct for the presence of absorption components. For some cases, however, the fluxes are clearly underestimated and this will be noted in the comments for individual objects in section 2.4.

Comparison of the absolute line fluxes measured from the spectra common to this atlas and the survey published by Ho, Filippenko \& Sargent (1997b) shows significant differences, with the data reported here being consistently underluminous with respect to the values found in Ho, Filippenko \& Sargent (1997b) by factors ranging between 3 and 5 (and up to a factor of 6 for observations acquired during the cloudy run on January 1997) Two reasons can be found for this discrepancy:

- poor weather conditions affected most of the observations reported here to a greater or lesser degree;

- aperture effects introduced by a different effective aperture $\left(1^{\prime \prime} \times 2^{\prime \prime}\right.$ for this project versus $2^{\prime \prime} \times 4^{\prime \prime}$ - Ho, Filippenko \& Sargent 1997b), which will mainly affect observations of resolved sources.

Comparison of relative line fluxes, however, shows a good agreement between both sets of data, as can be seen in table 3.5, where the ratio of emission line fluxes to the $\mathrm{H} \alpha$ flux are shown. The objects in table 3.5 were chosen because of their clean HII region spectrum with very little contamination from an underlying stellar component, so that the comparison with the (stellar subtracted) data from Ho, Filippenko \& Sargent (1997b) was appropiate. 


\begin{tabular}{|ll|cccc|}
\hline \hline Galaxy & & H $\beta \lambda 4861$ & {$[\mathrm{OIII}] \lambda .5007$} & {$[\mathrm{NII}] \lambda 6584$} & {$[\mathrm{SII}] \lambda 6730$} \\
\hline IC 342 & Atlas & 0.09 & - & 0.54 & 0.15 \\
& HFS & 0.13 & 0.01 & 0.45 & 0.12 \\
NGC 1560 & Atlas & 0.27 & 0.84 & 0.05 & 0.05 \\
& HFS & 0.24 & 0.66 & 0.07 & 0.06 \\
NGC 2976 & Atlas & 0.23 & 0.29 & 0.29 & 0.09 \\
& HFS & 0.25 & 0.36 & 0.31 & 0.08 \\
NGC 3738 & Atlas & 0.41 & 1.10 & 0.12 & 0.19 \\
& HFS & 0.38 & 1.14 & 0.10 & 0.13 \\
NGC 6946 & Atlas & 0.07 & - & 0.63 & 0.18 \\
& HFS & 0.07 & 0.03 & 0.64 & 0.16 \\
\hline \hline
\end{tabular}

Table 3.5: Fluxes (relative to $\mathrm{H} \alpha \lambda 6563$ ) given in this atlas and published by Ho, Filippenko \& Sargent (1997b) - HFS. 


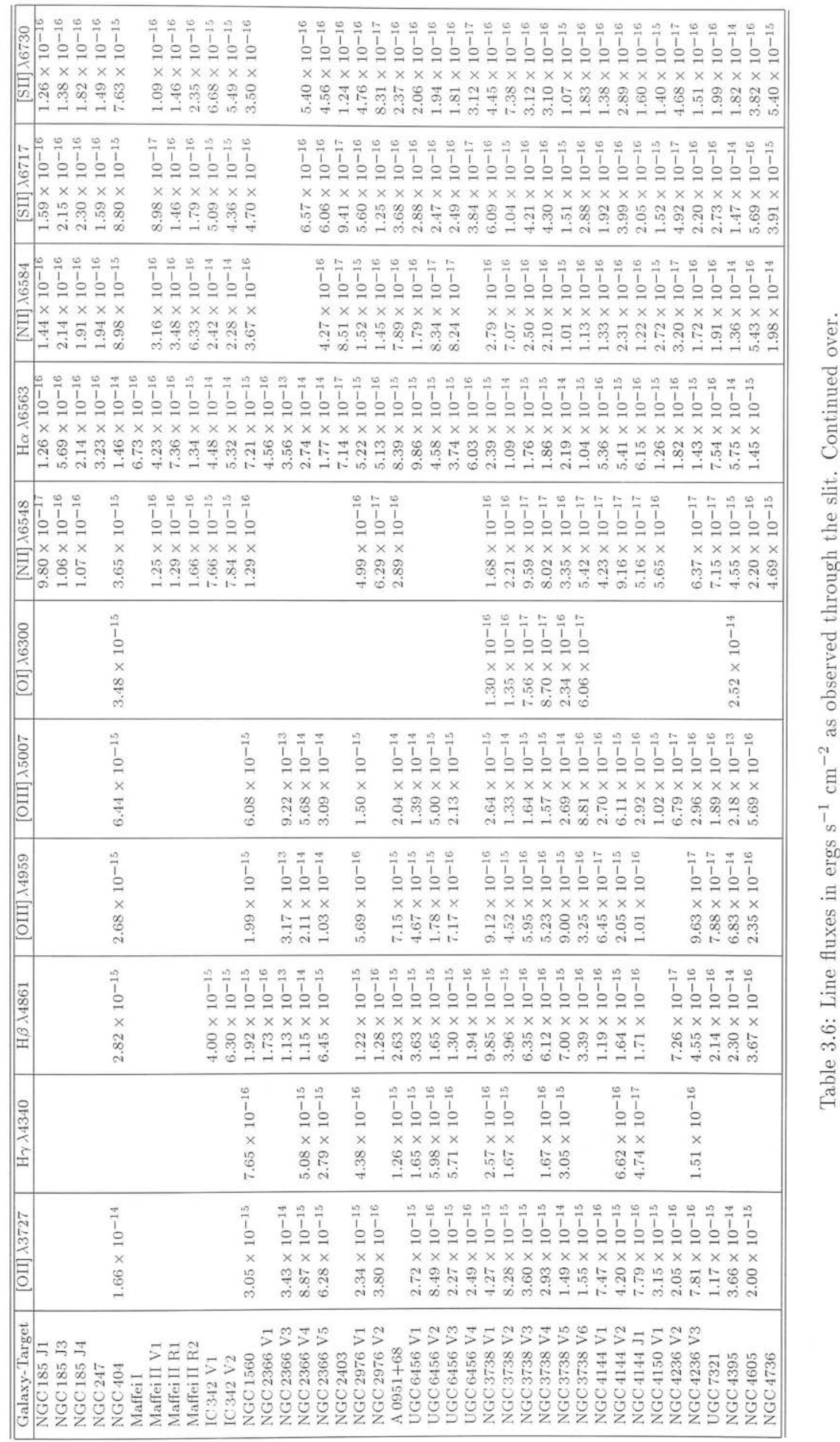




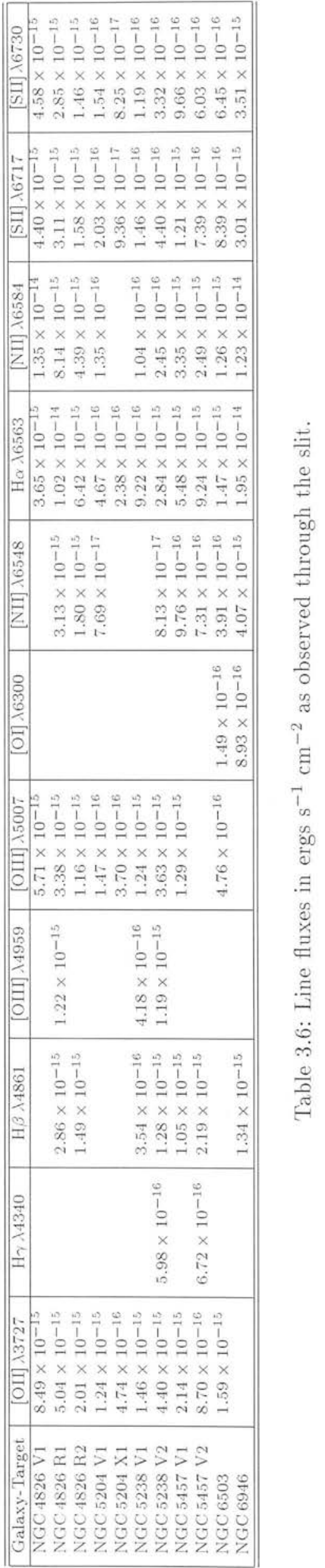



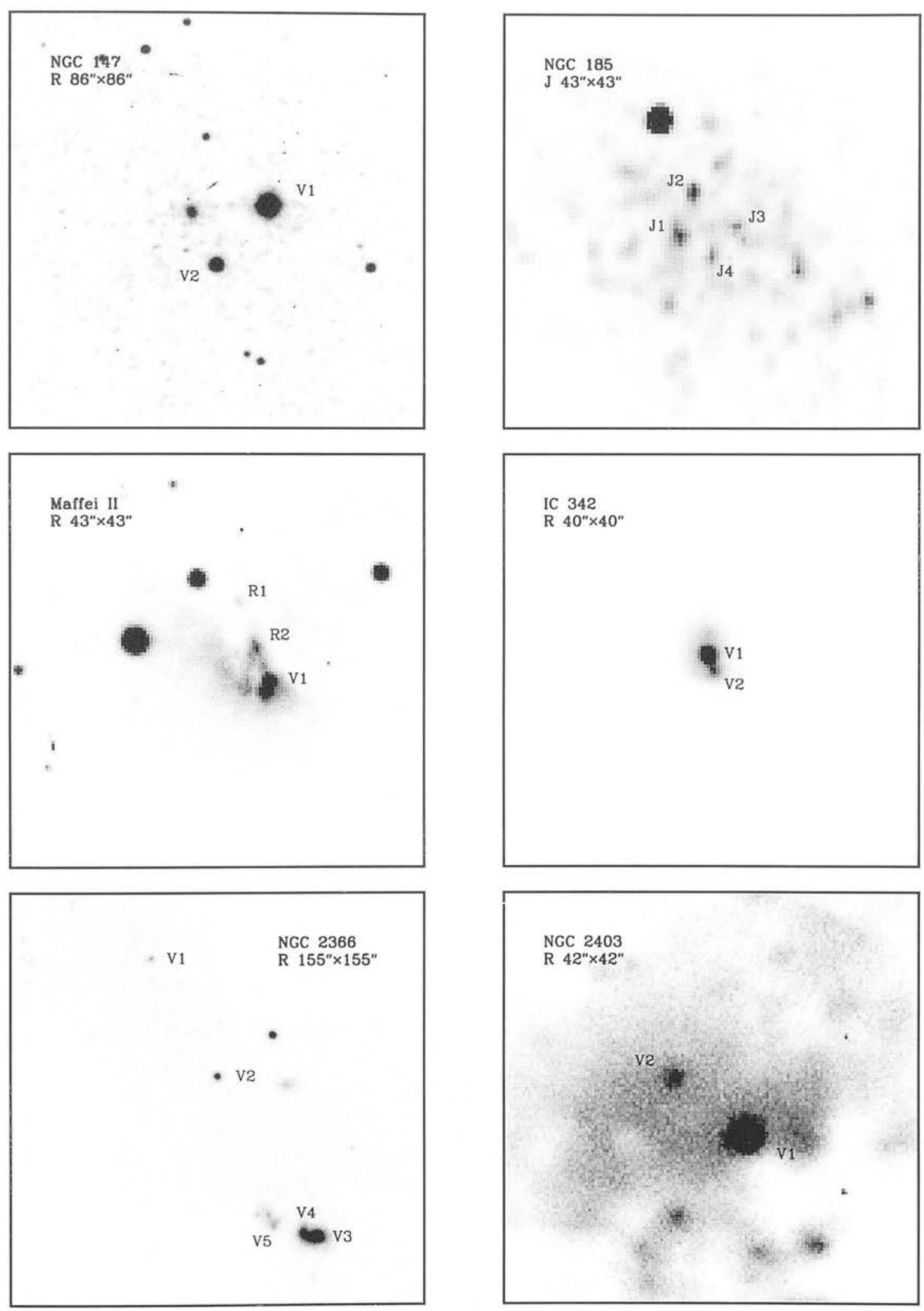

Figure 3.5: Nuclear spectroscopy targets. 

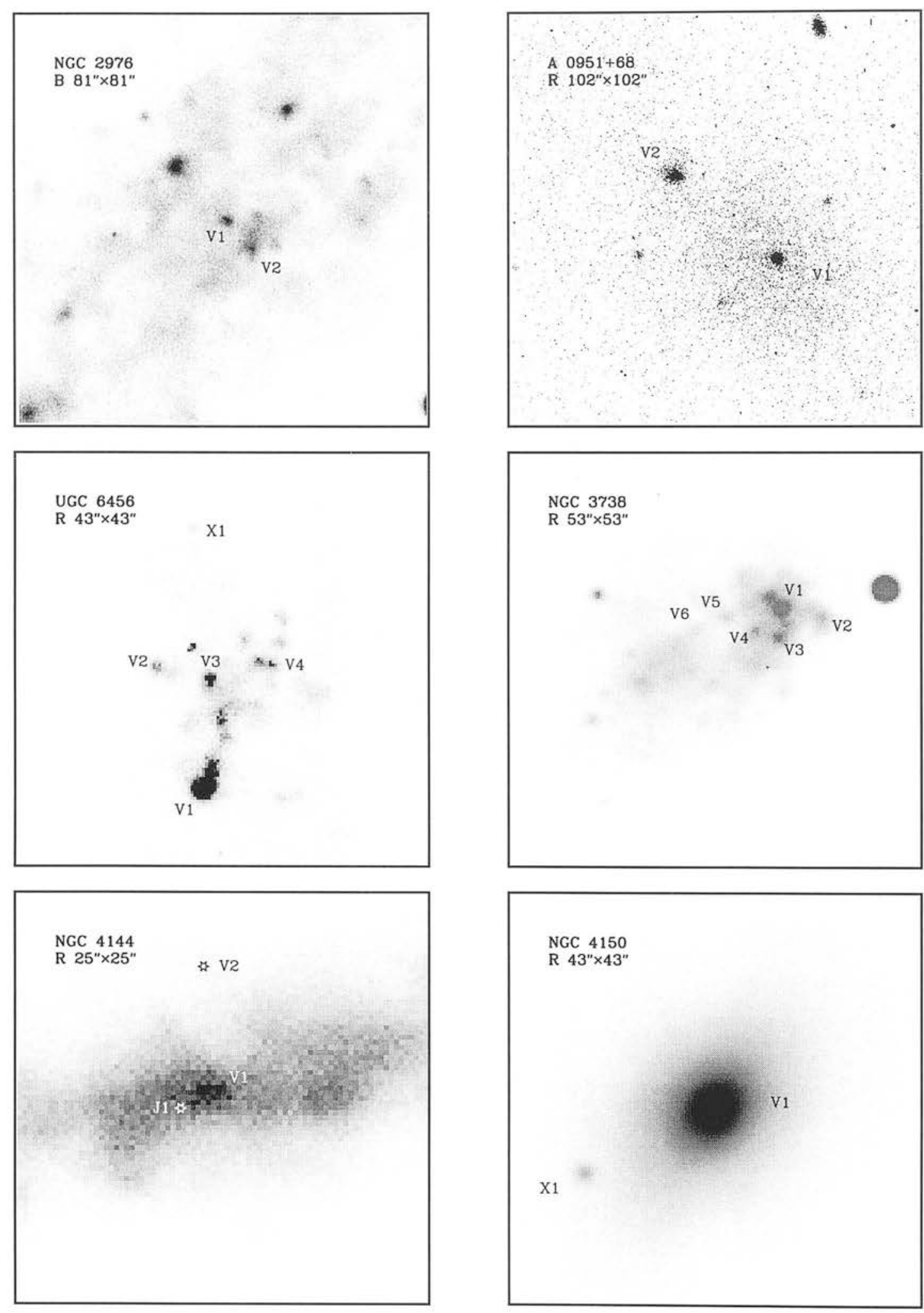

Figure 3.6: Nuclear spectroscopy targets. 

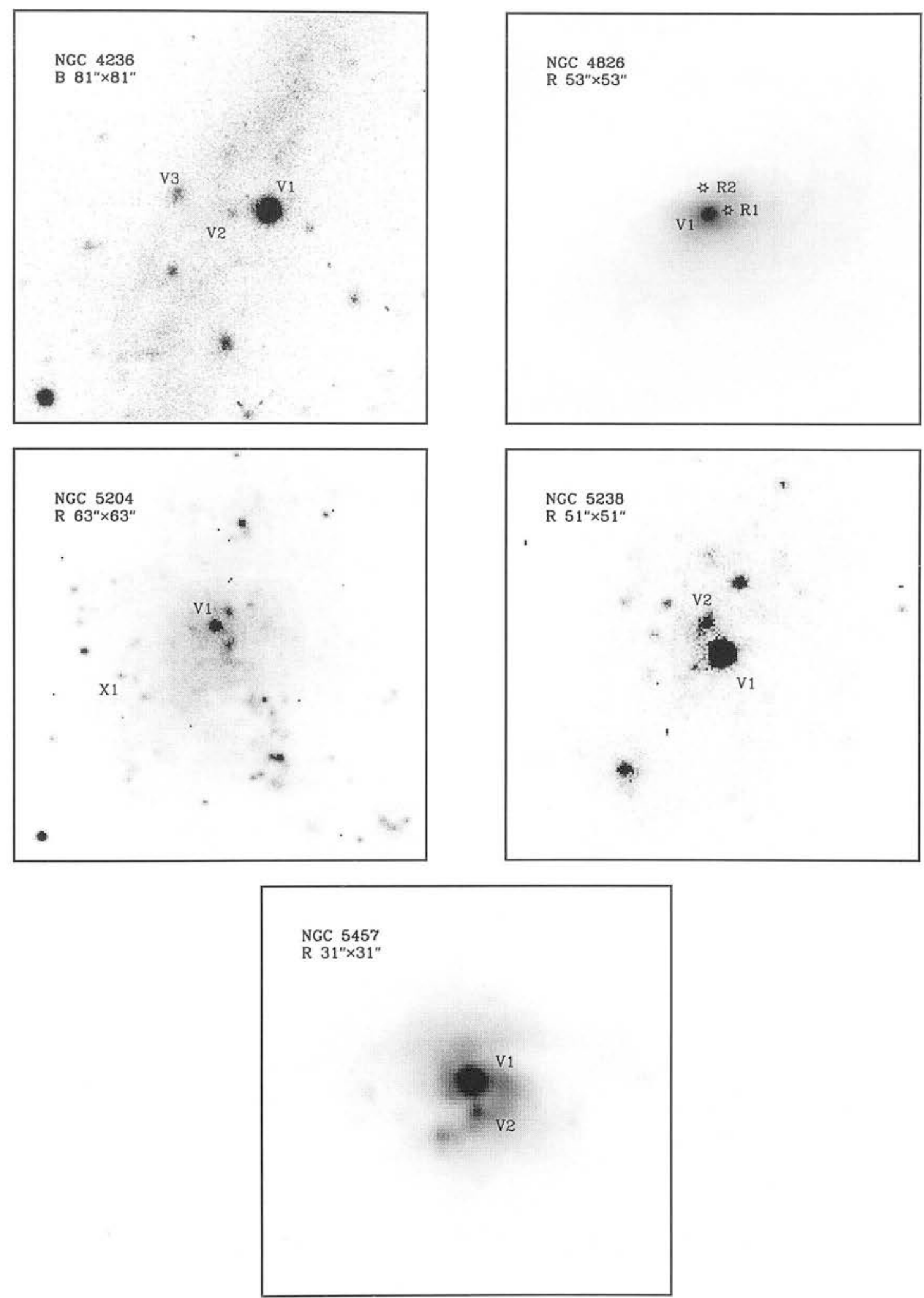

Figure 3.7: Nuclear spectroscopy targets. 


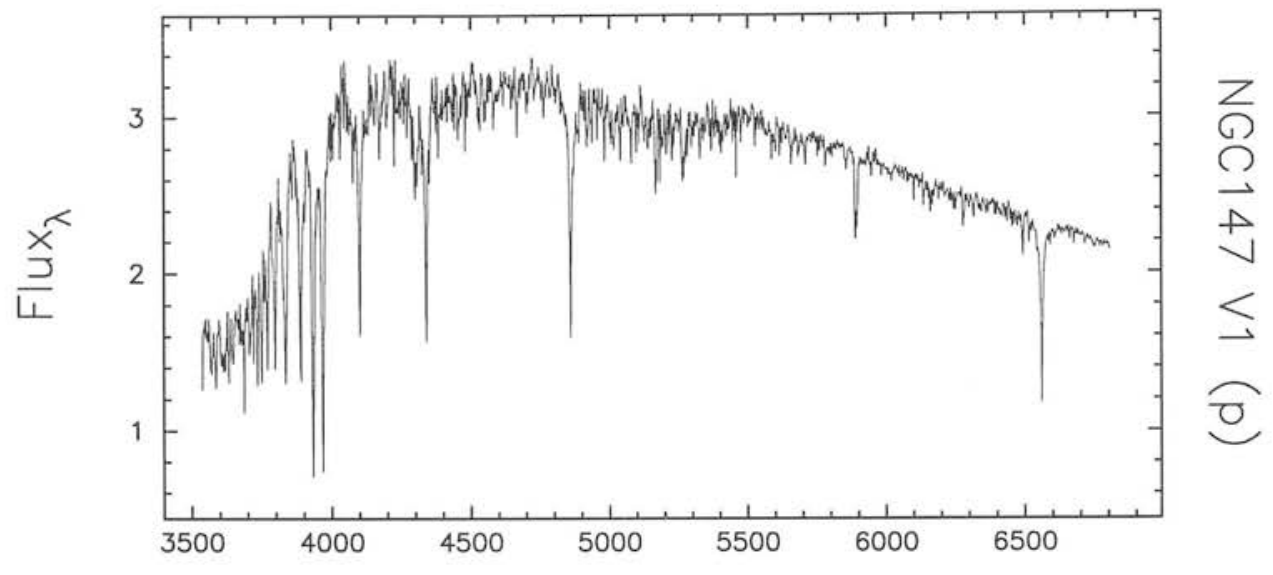

Angstroms

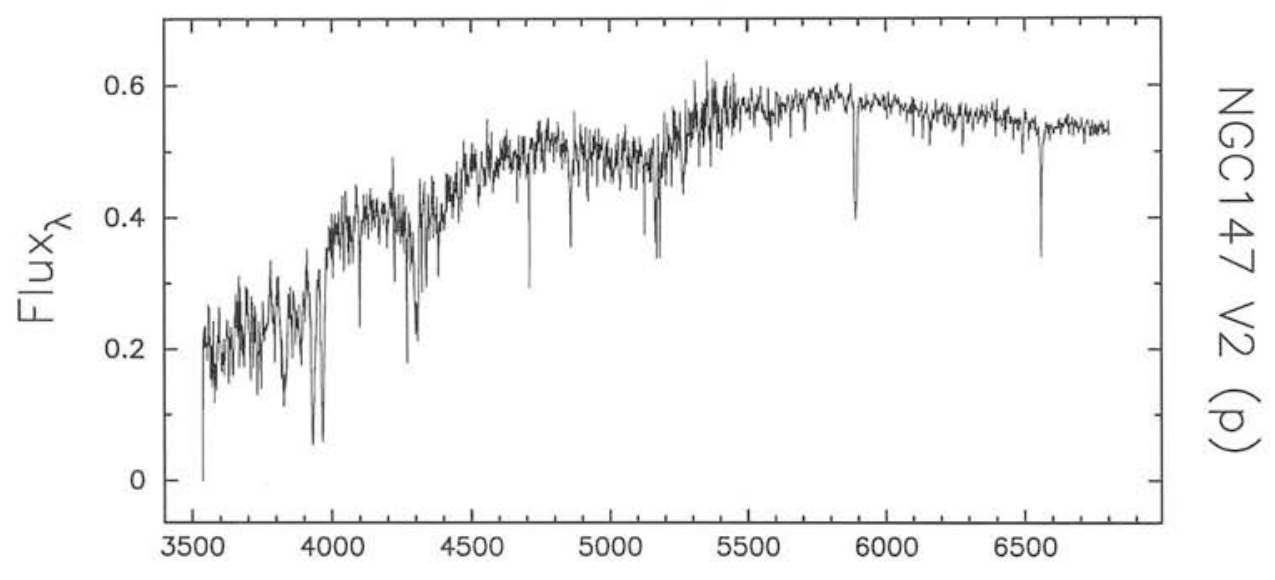

Angstroms

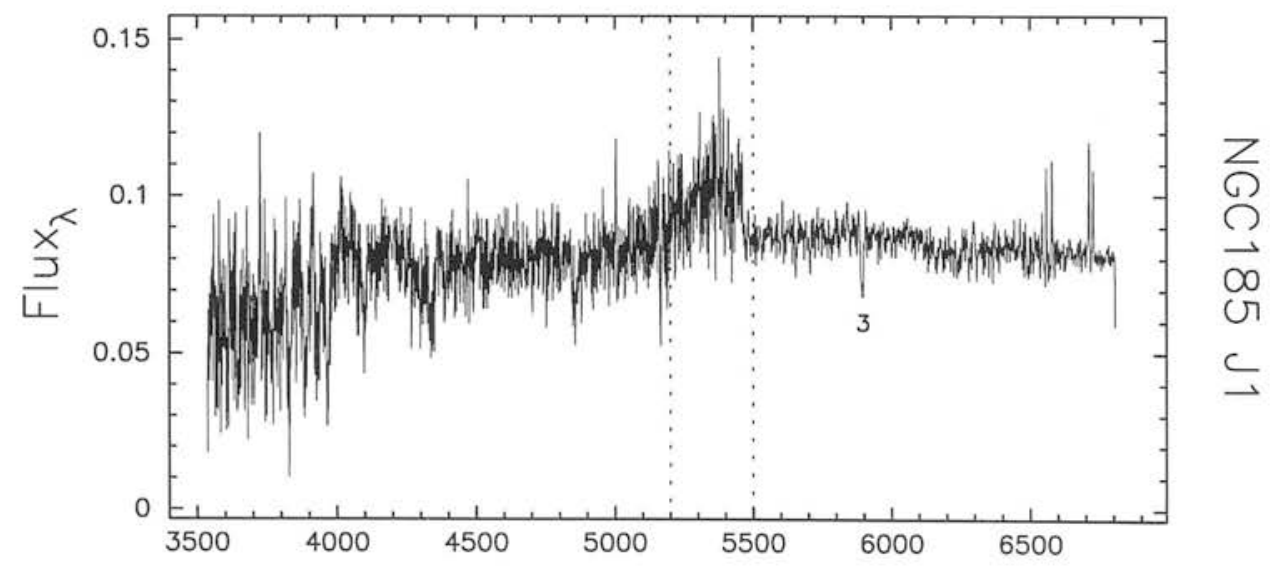

Angstroms

Figure 3.8: Flux in $10^{-15} \mathrm{ergs} \mathrm{s}^{-1} \mathrm{~cm}^{-2} \AA^{-1}$. 


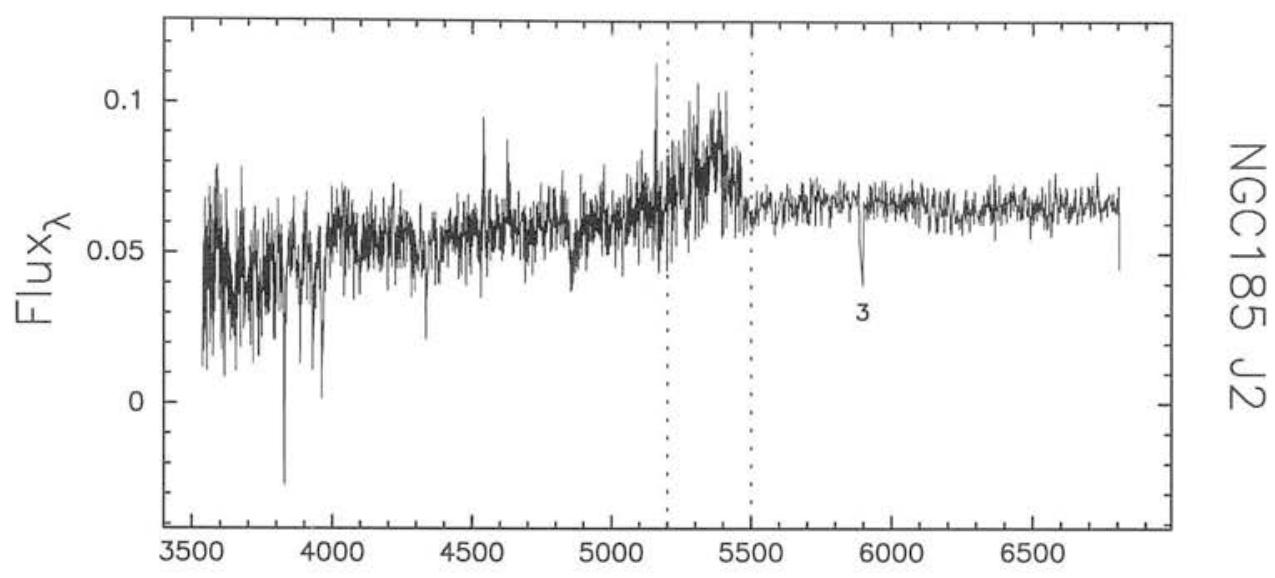

Angstroms

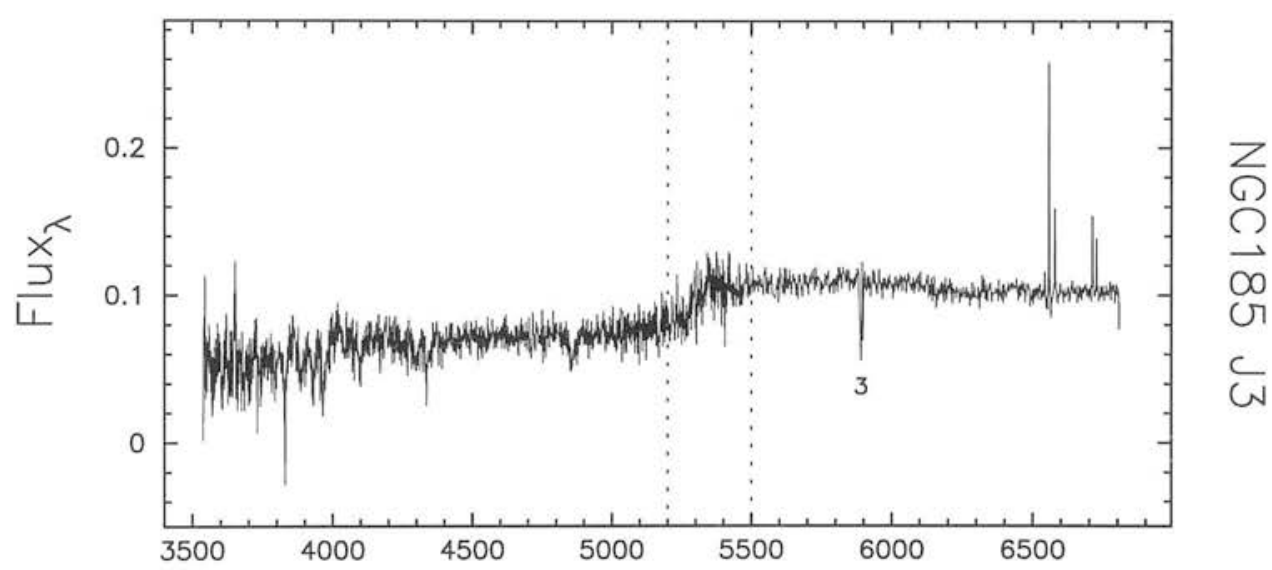

Angstroms

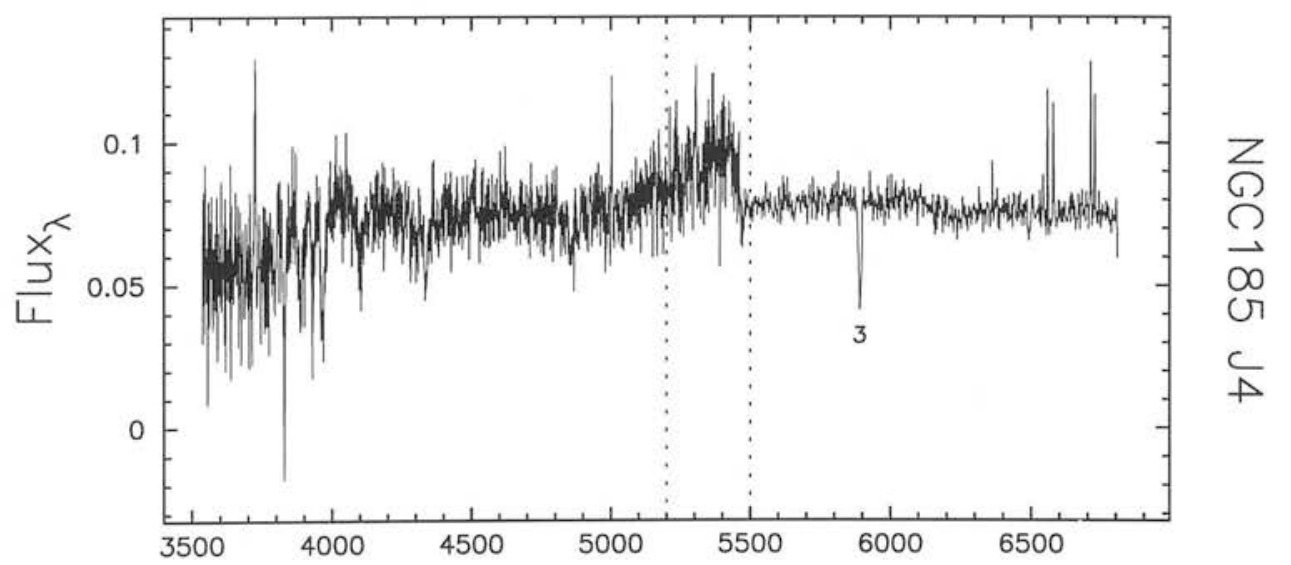

\section{Angstroms}

Figure 3.9: Flux in $10^{-15} \mathrm{ergs} \mathrm{s}^{-1} \mathrm{~cm}^{-2} \AA^{-1}$. 


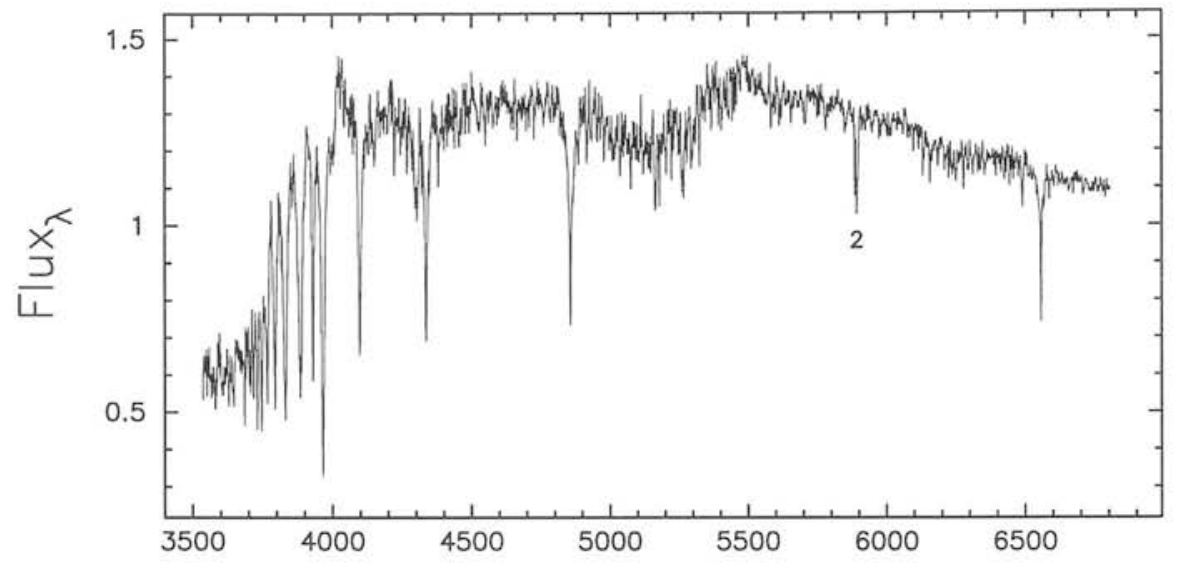

$z$
O
N
G

Angstroms

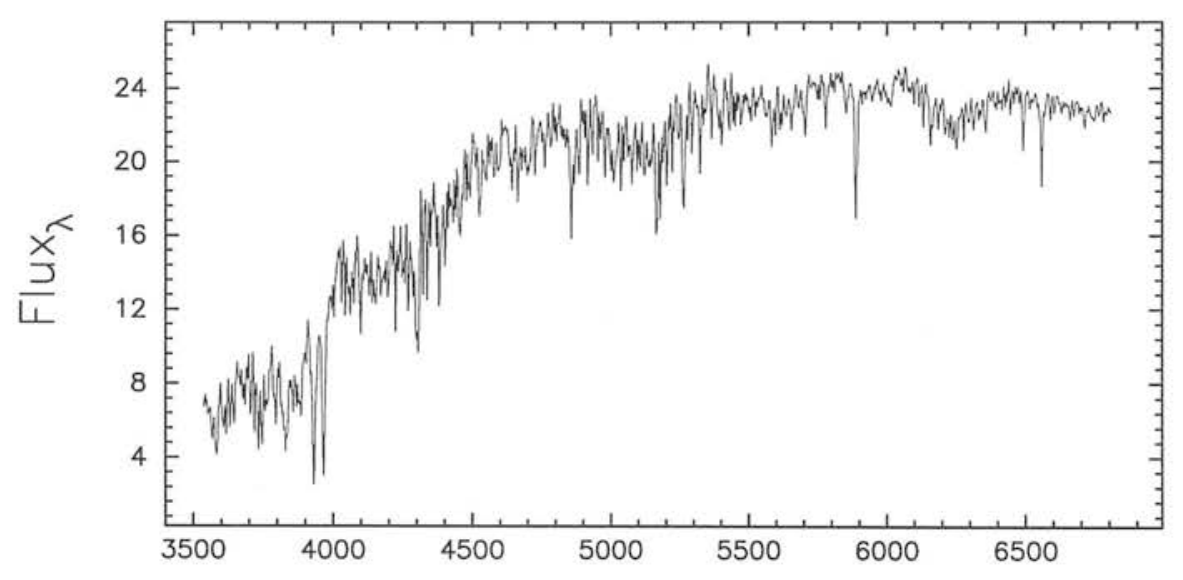

$Z$
N
N

Angstroms

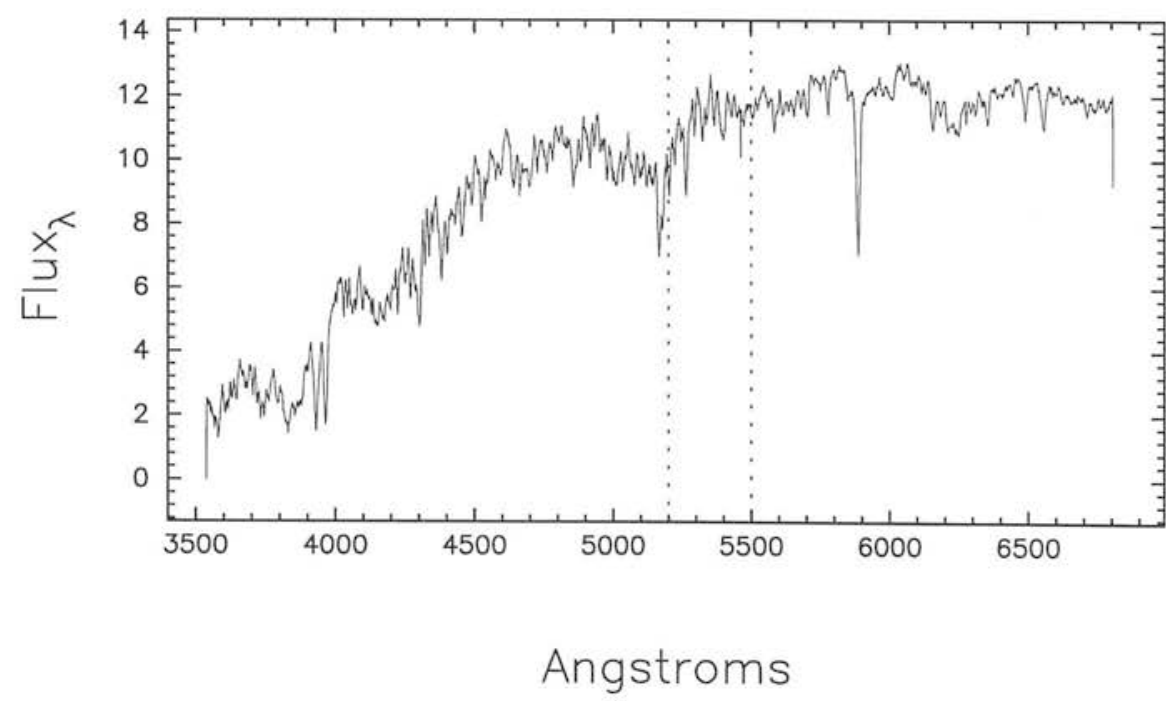

$Z$
$N$
$N$
$N$
$N$

Figure 3.10: Flux in $10^{-15} \mathrm{ergs} \mathrm{s}^{-1} \mathrm{~cm}^{-2} \mathrm{~A}^{-1}$. 


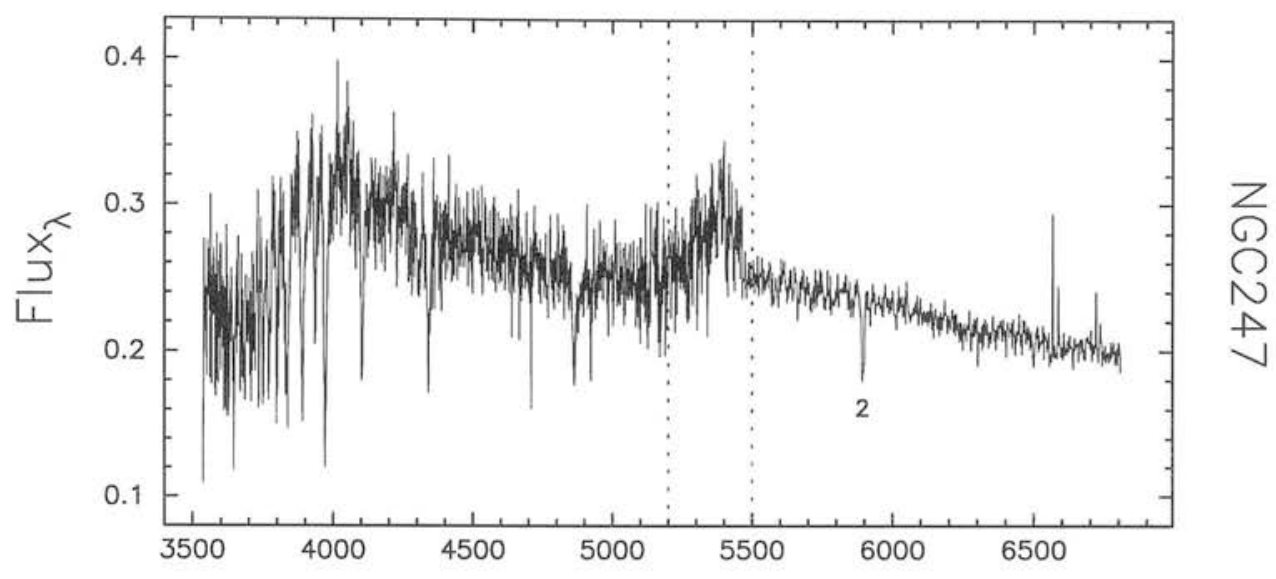

Angstroms

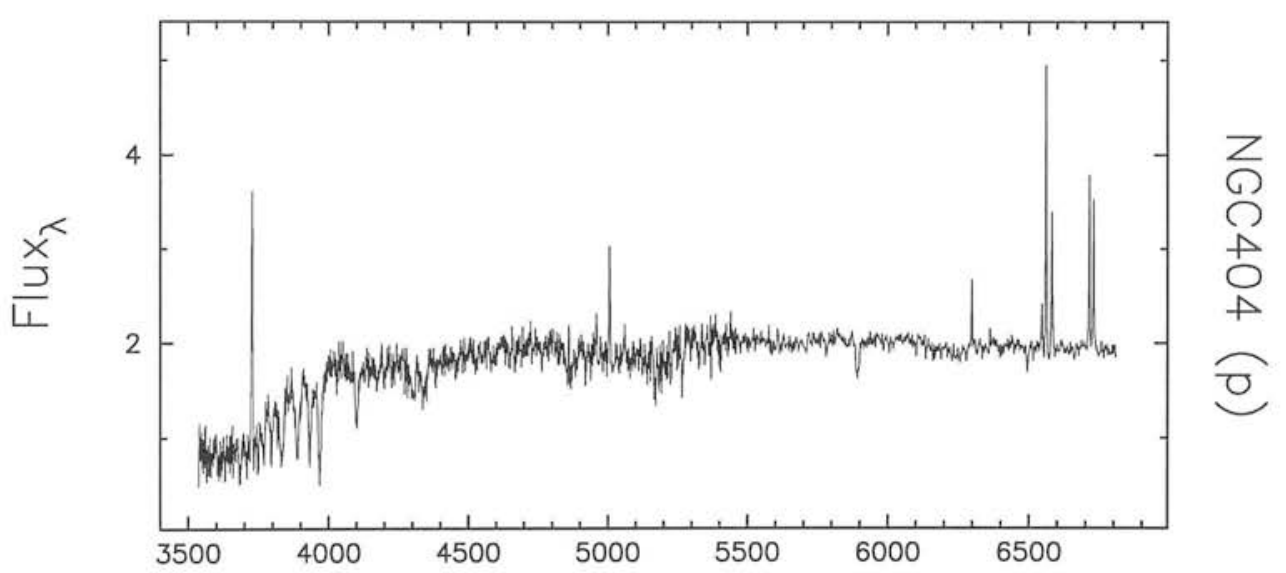

Angstroms

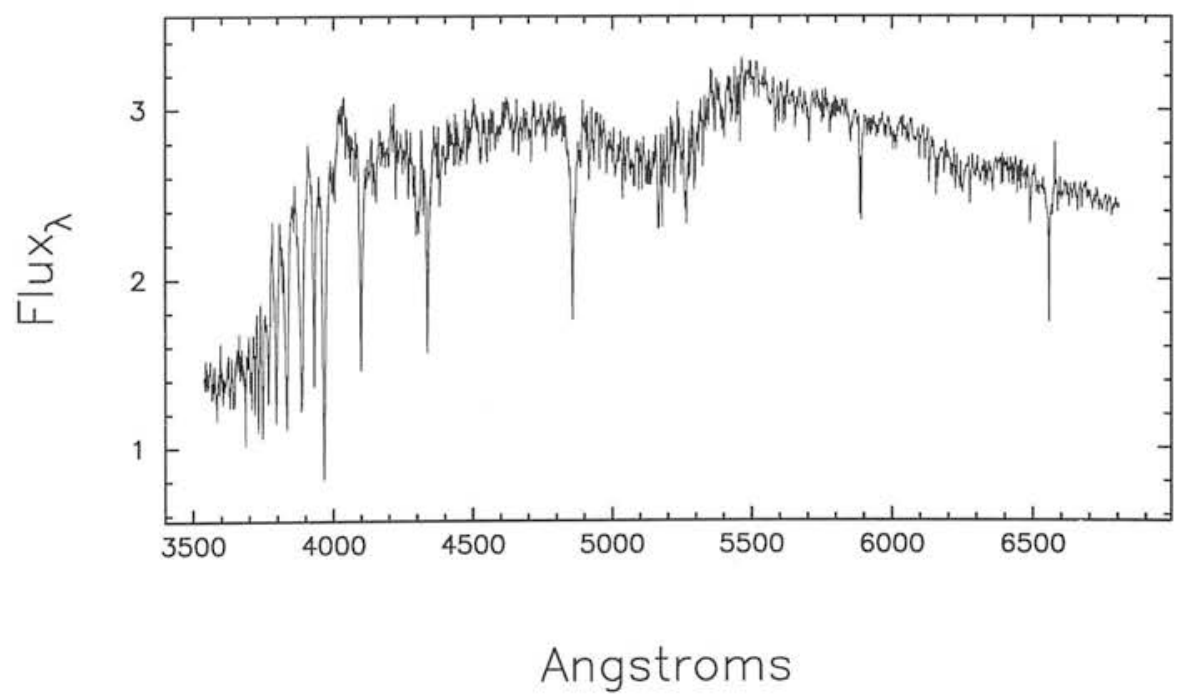

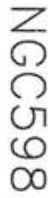

Figure 3.11: Flux in $10^{-15} \mathrm{ergs} \mathrm{s}^{-1} \mathrm{~cm}^{-2} \AA^{-1}$. 


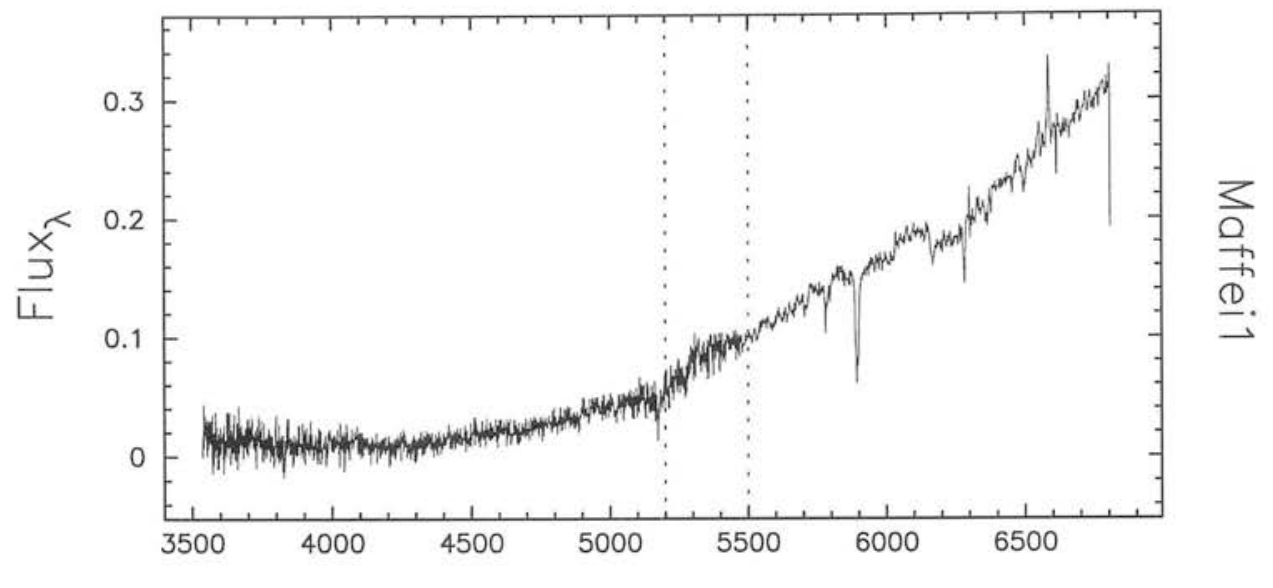

Angstroms

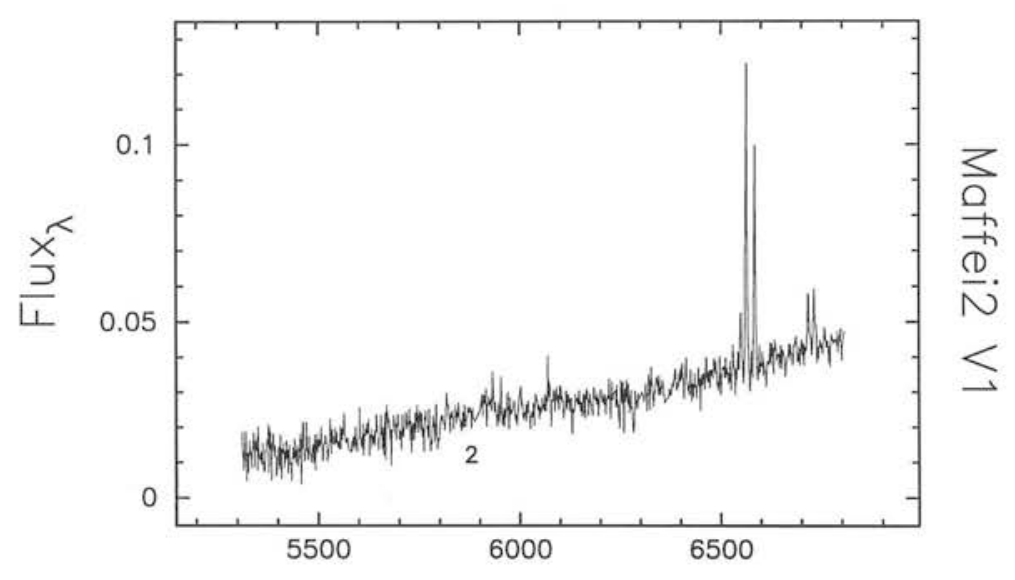

Angstroms

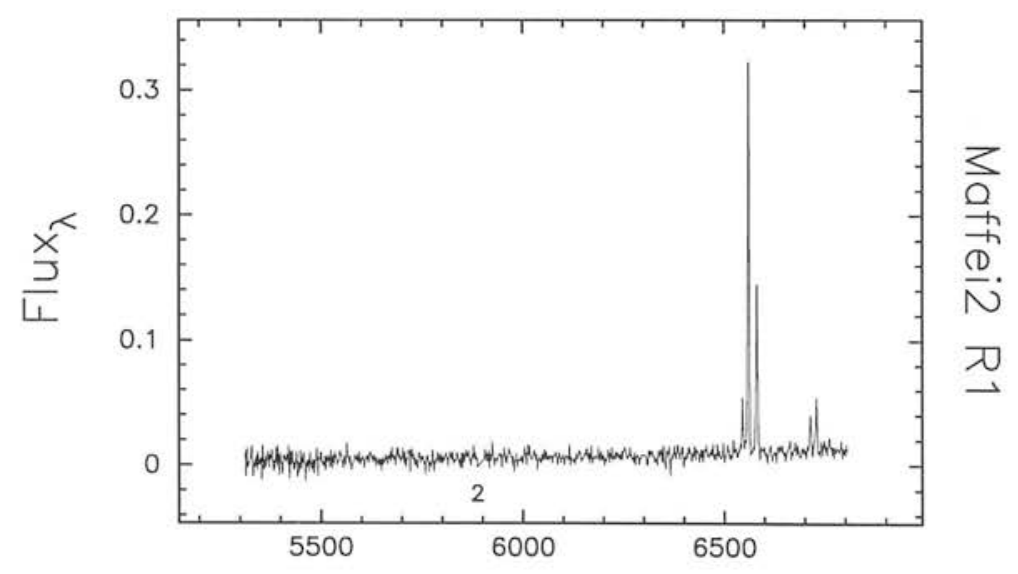

\section{Angstroms}

Figure 3.12: Flux in $10^{-15} \mathrm{ergs} \mathrm{s}^{-1} \mathrm{~cm}^{-2} \AA^{-1}$. 


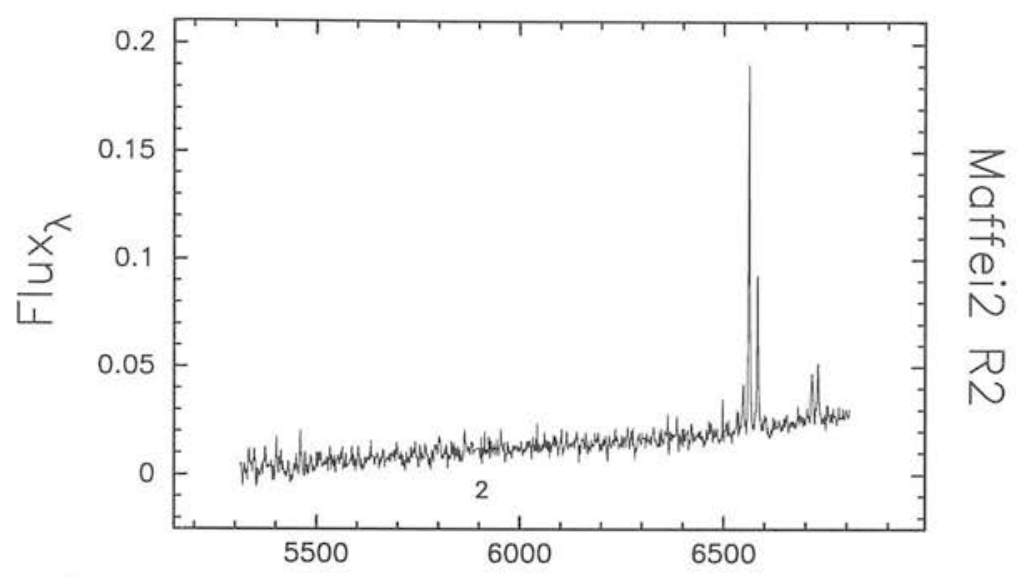

Angstroms

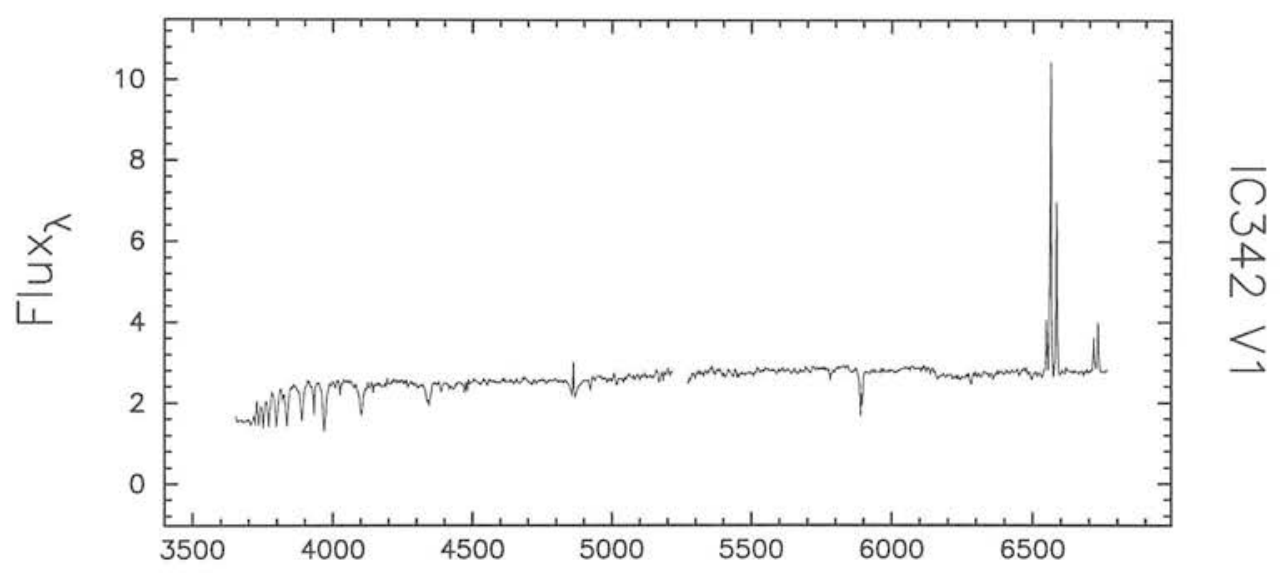

Angstroms

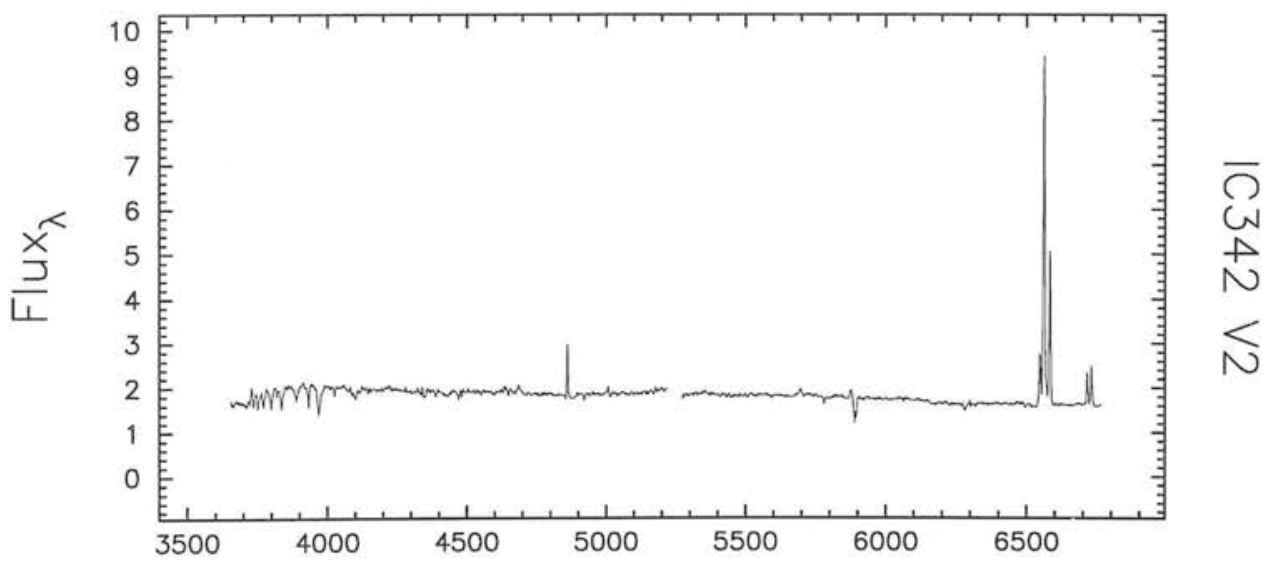

Angstroms

Figure 3.13: Flux in $10^{-15} \mathrm{ergs} \mathrm{s}^{-1} \mathrm{~cm}^{-2} \AA^{-1}$. 


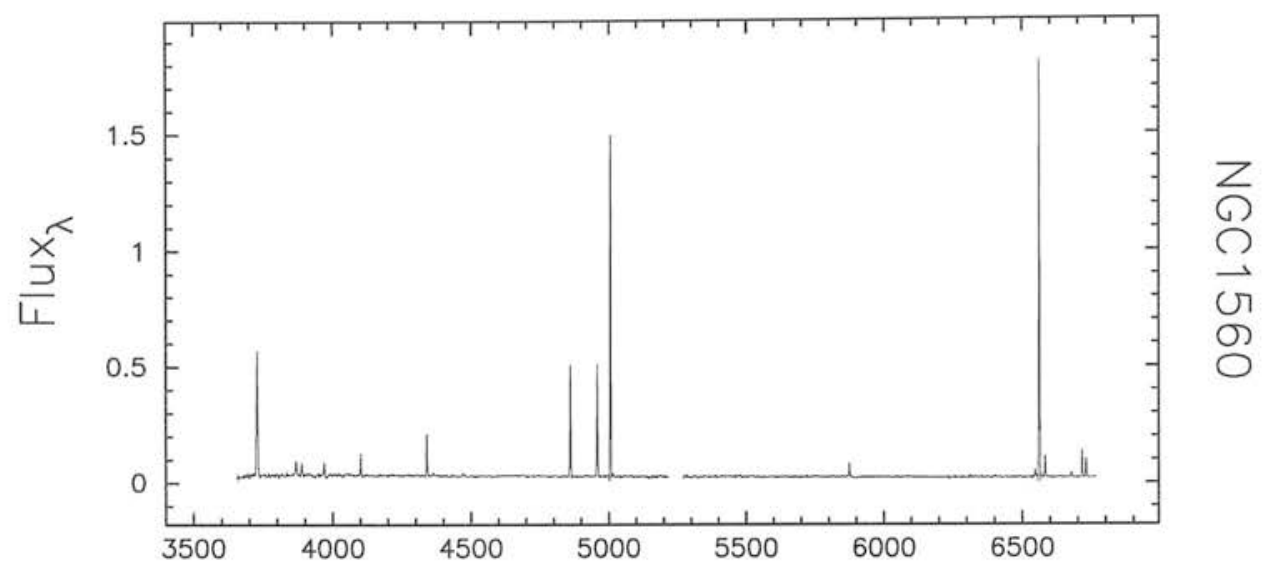

Angstroms

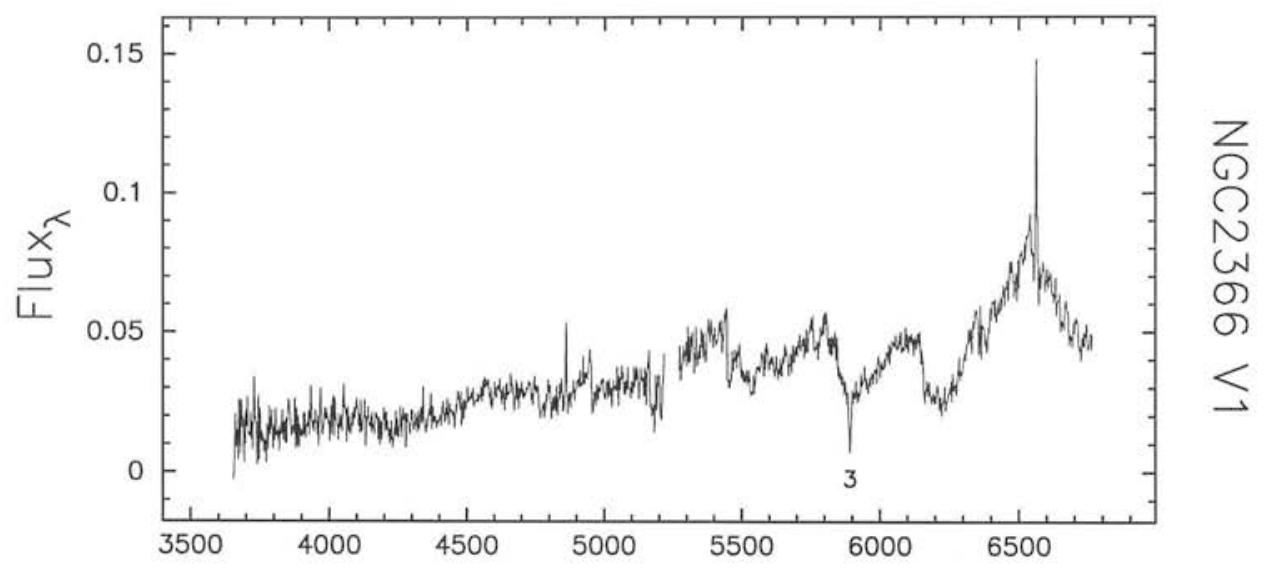

Angstroms

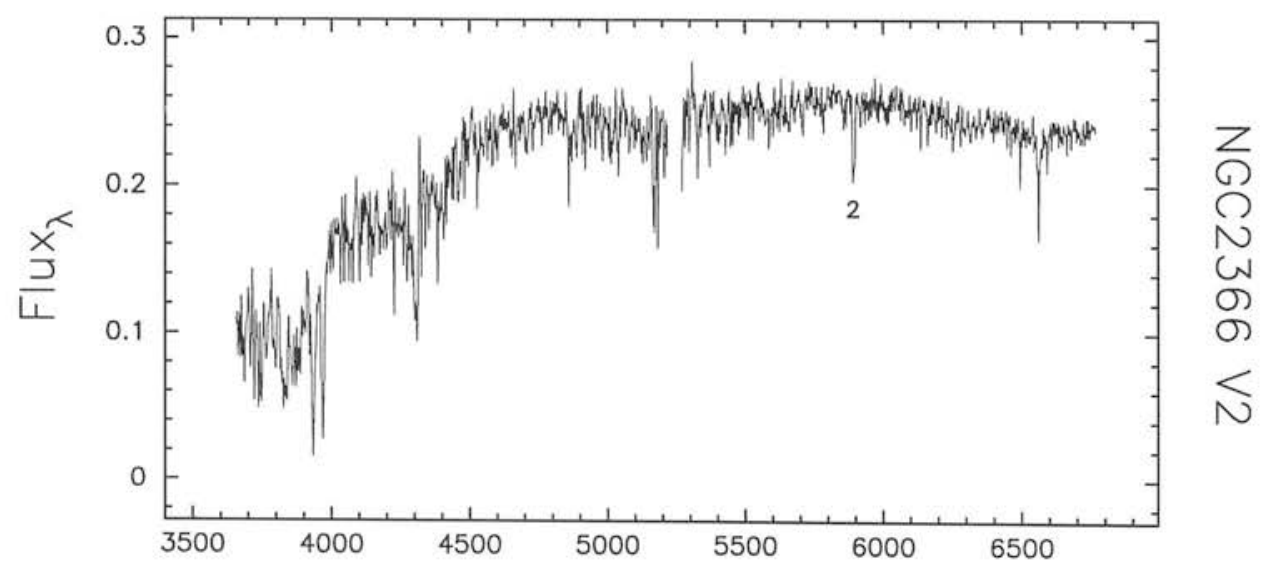

\section{Angstroms}

Figure 3.14: Flux in $10^{-15} \mathrm{ergs} \mathrm{s}^{-1} \mathrm{~cm}^{-2} \AA^{-1}$. 


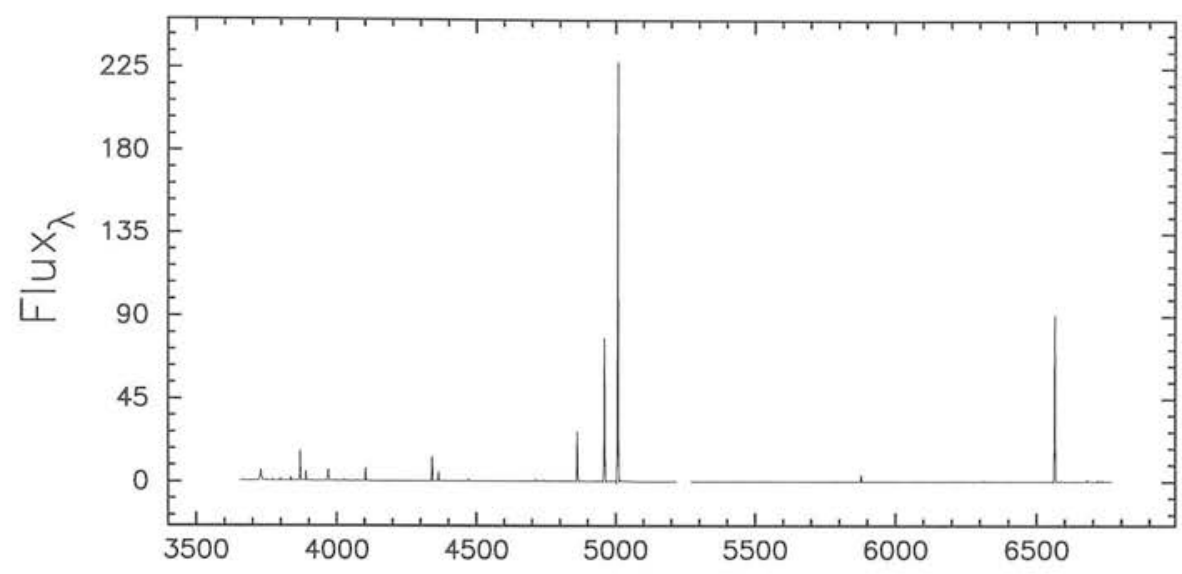

$z$
$\Omega$
$N$
$\omega$
$\sigma$
$\sigma$
$\omega$

Angstroms

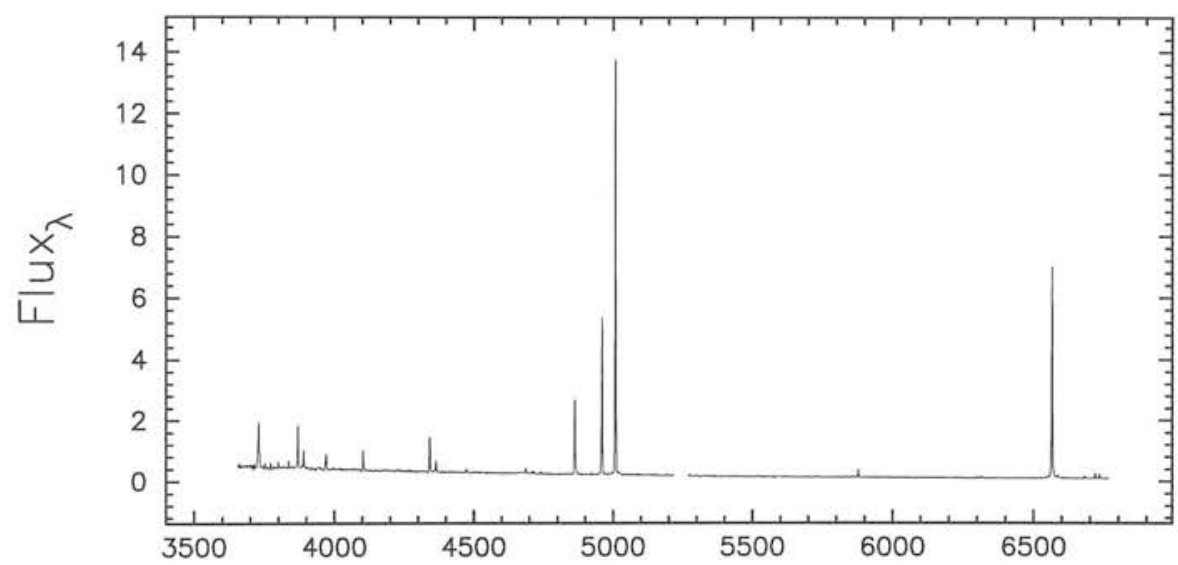

$z$
$\Omega$
$N$
$w$
$\Omega$
$\sigma$
๙

Angstroms

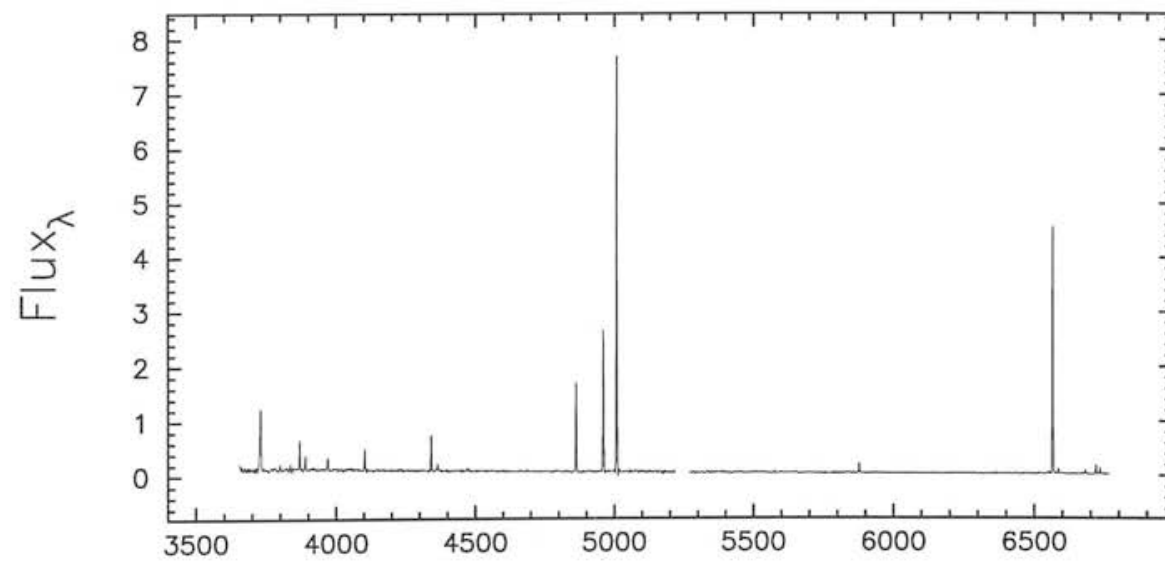

$Z$
$\Omega$
$N$
$w$
$\sigma$
$\sigma$
जा

Angstroms

Figure 3.15: Flux in $10^{-15} \mathrm{ergs} \mathrm{s}^{-1} \mathrm{~cm}^{-2} \AA^{-1}$. 


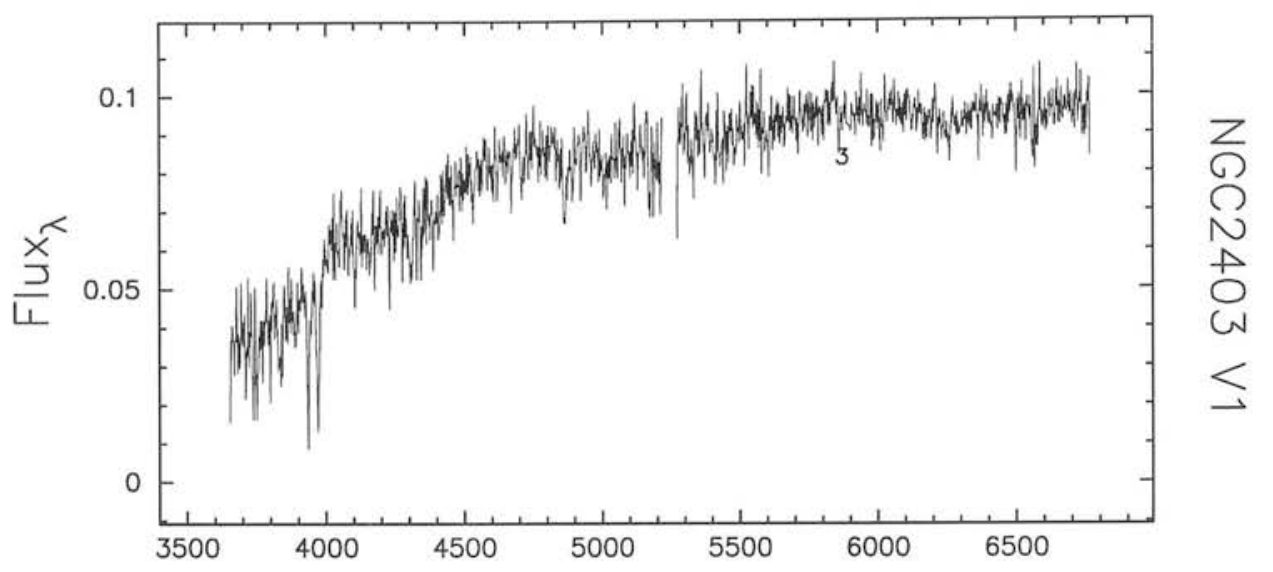

Angstroms

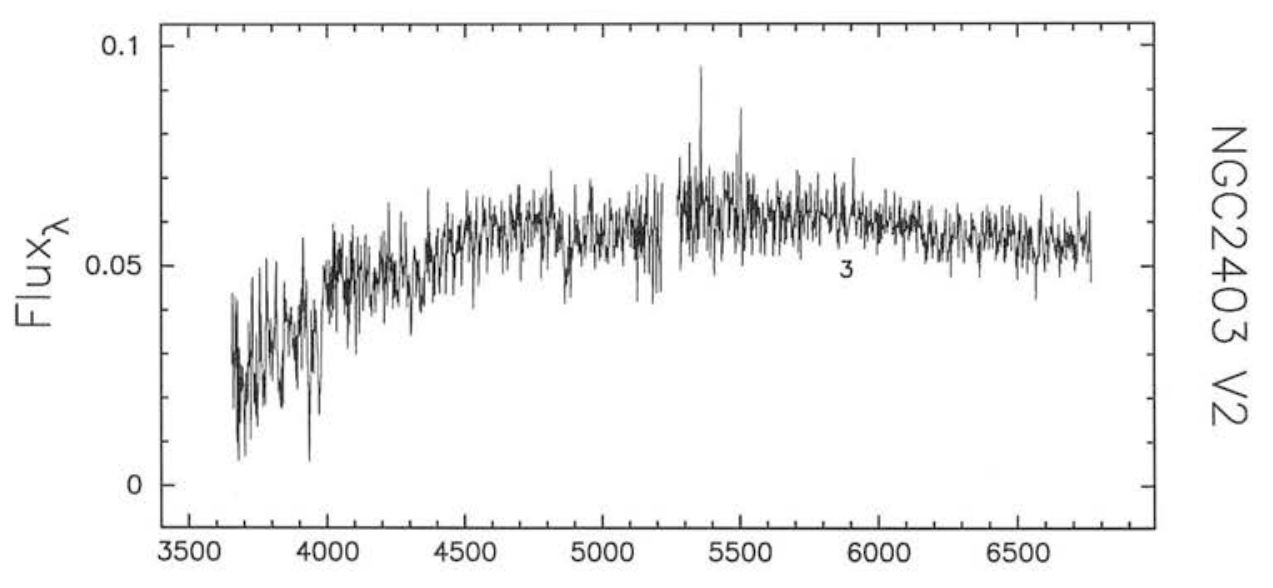

Angstroms

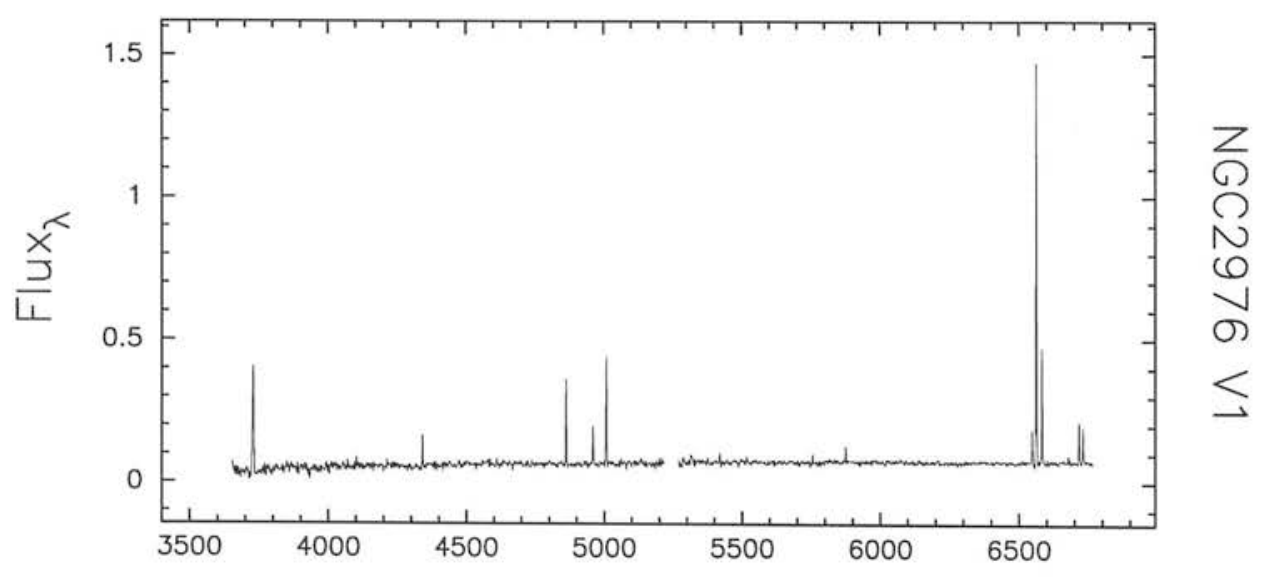

Angstroms

Figure 3.16: Flux in $10^{-15} \mathrm{ergs} \mathrm{s}^{-1} \mathrm{~cm}^{-2} \AA^{-1}$. 


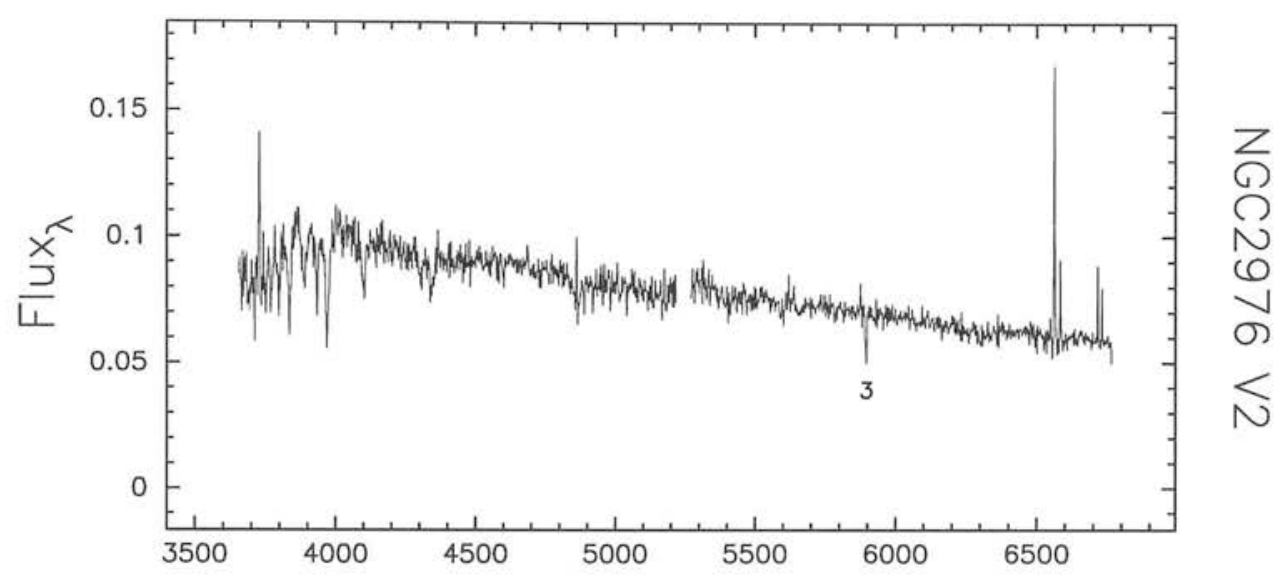

Angstroms

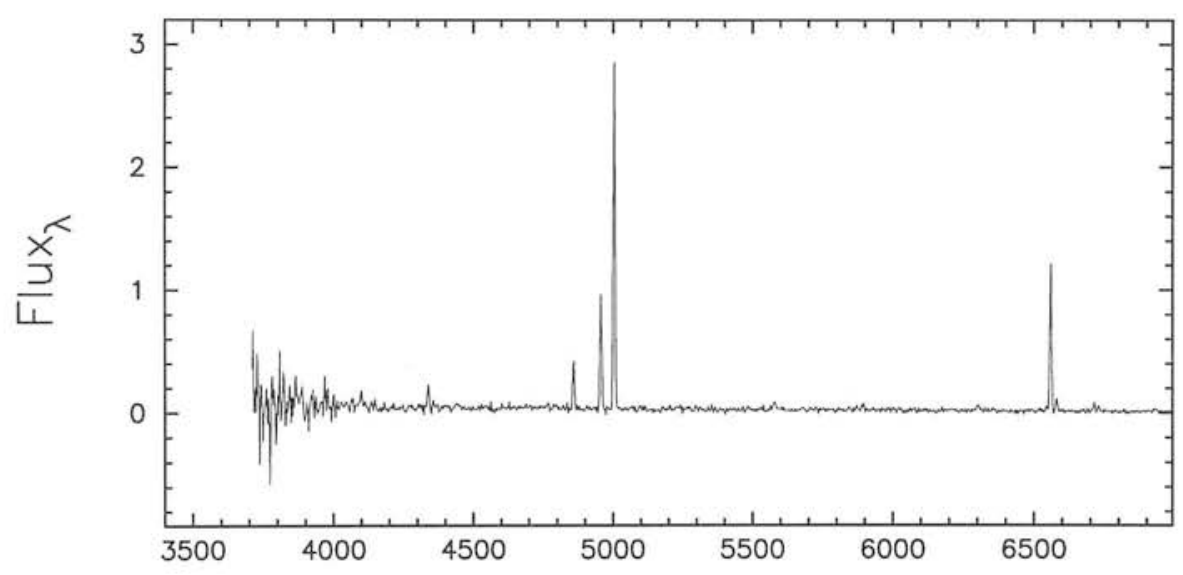

$D$
0
0
U
$\overrightarrow{+}$
0
$\infty$
$\hat{N}$

Angstroms

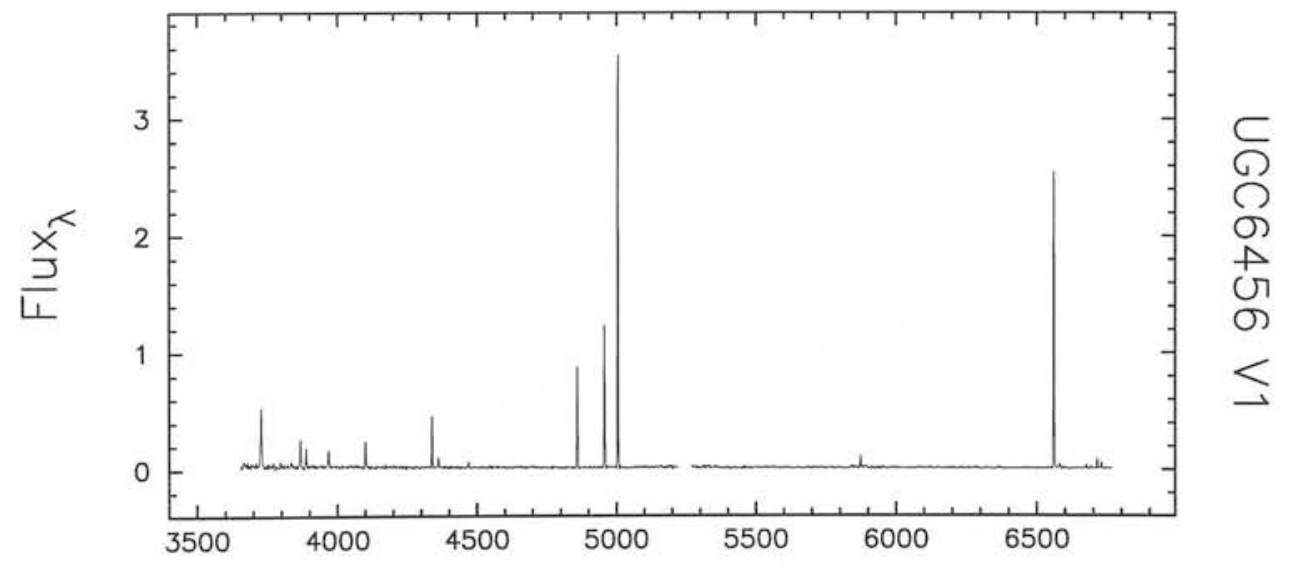

Angstroms

Figure 3.17: Flux in $10^{-15} \mathrm{ergs} \mathrm{s}^{-1} \mathrm{~cm}^{-2} \AA^{-1}$. 


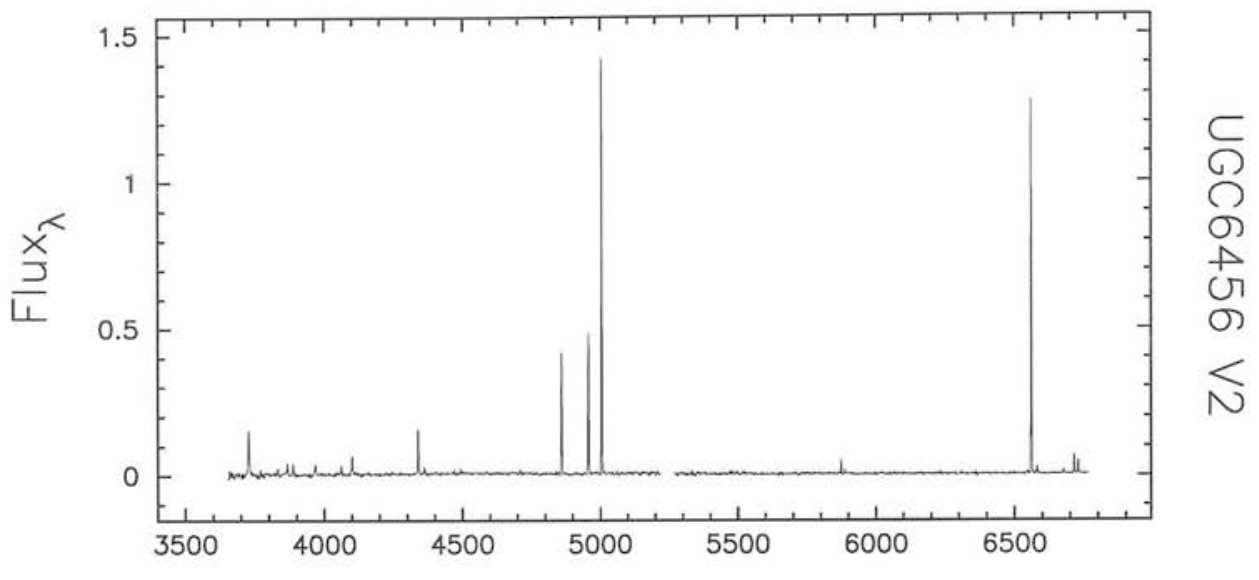

Angstroms

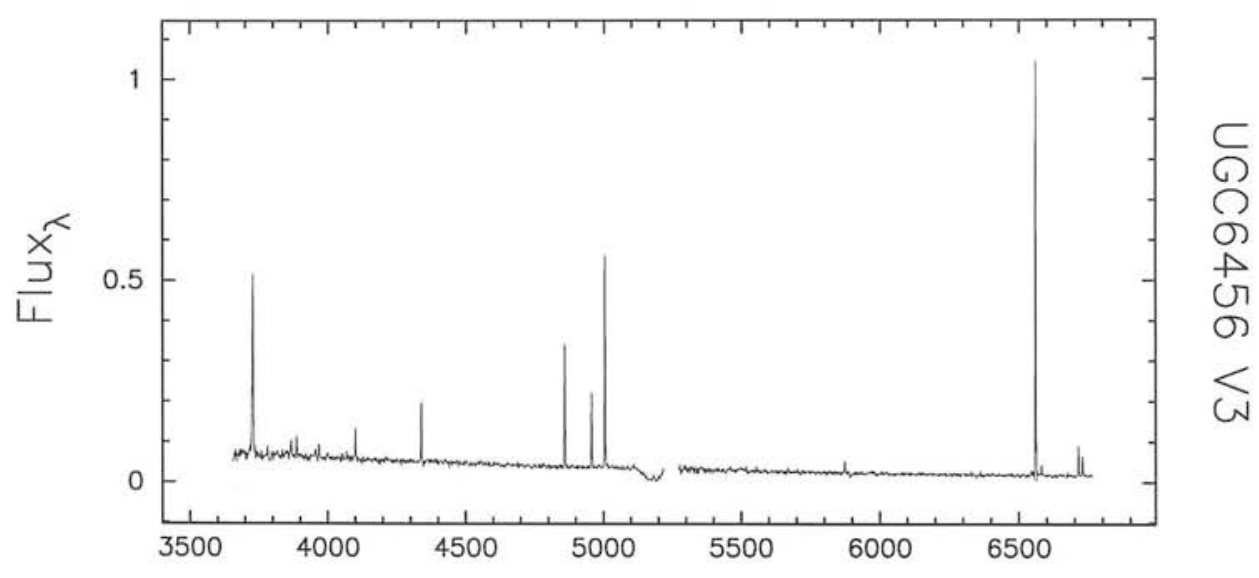

Angstroms

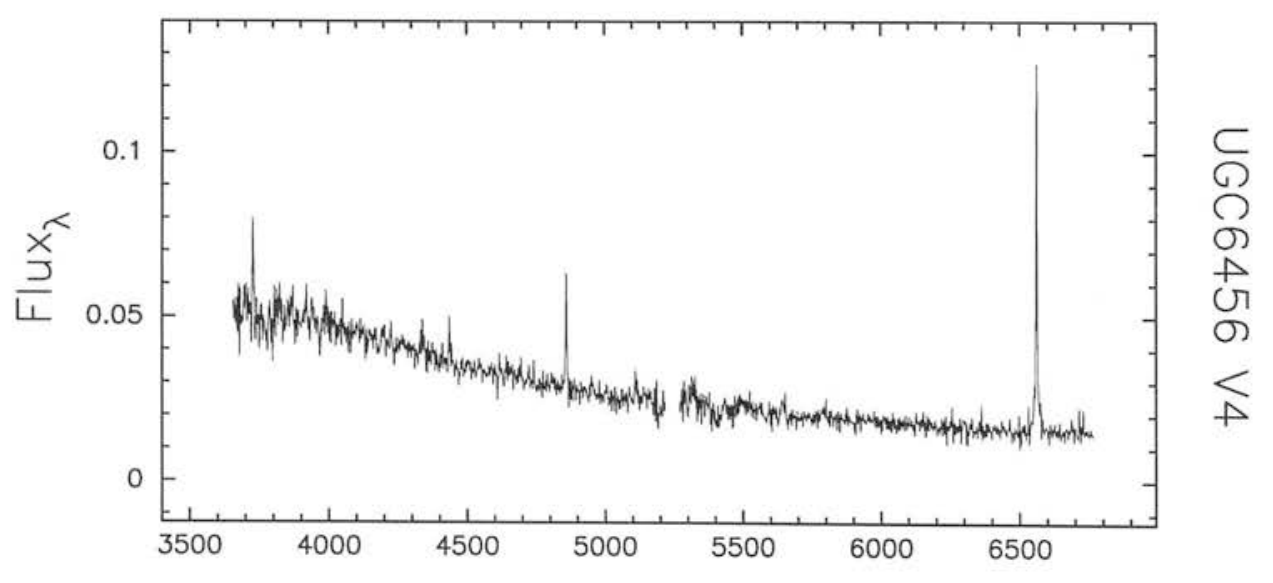

Angstroms

Figure 3.18: Flux in $10^{-15} \mathrm{ergs} \mathrm{s}^{-1} \mathrm{~cm}^{-2} \AA^{-1}$. 


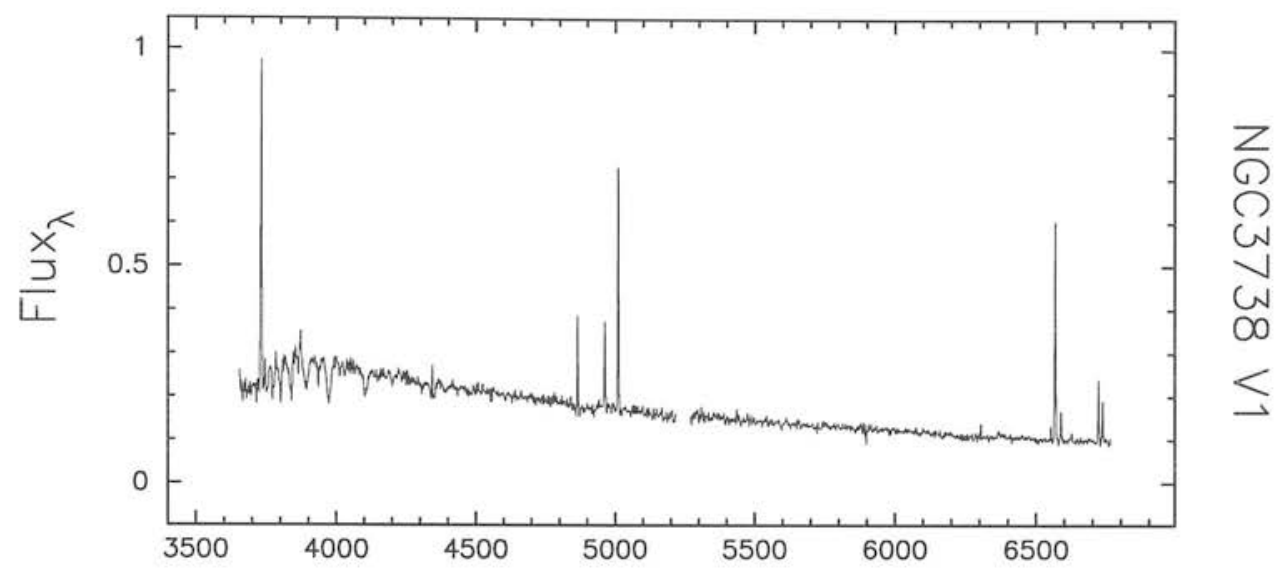

Angstroms

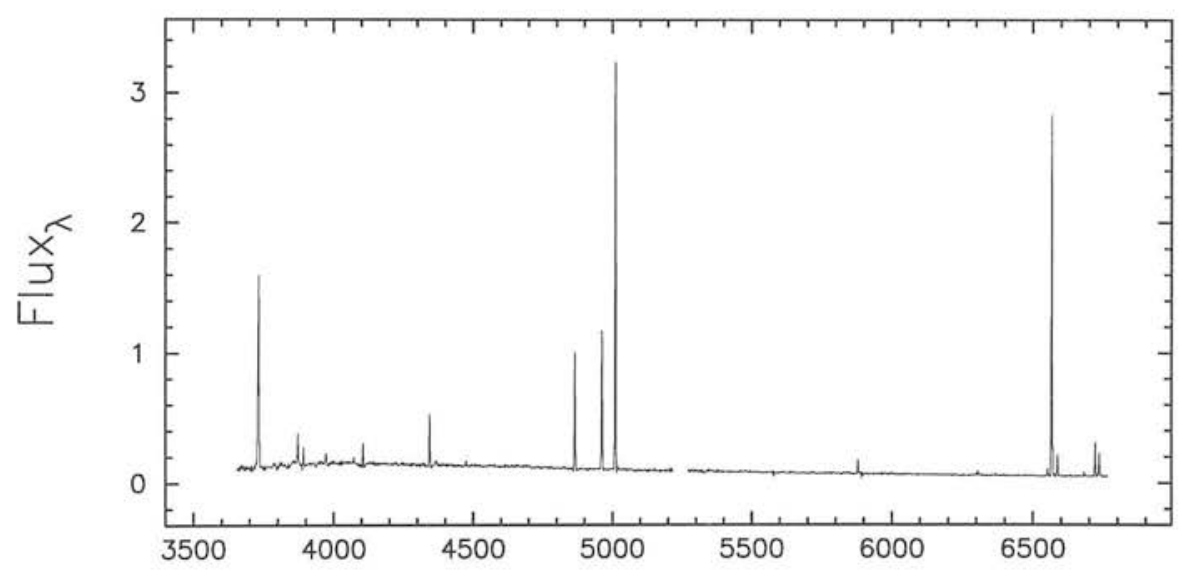

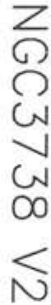

Angstroms

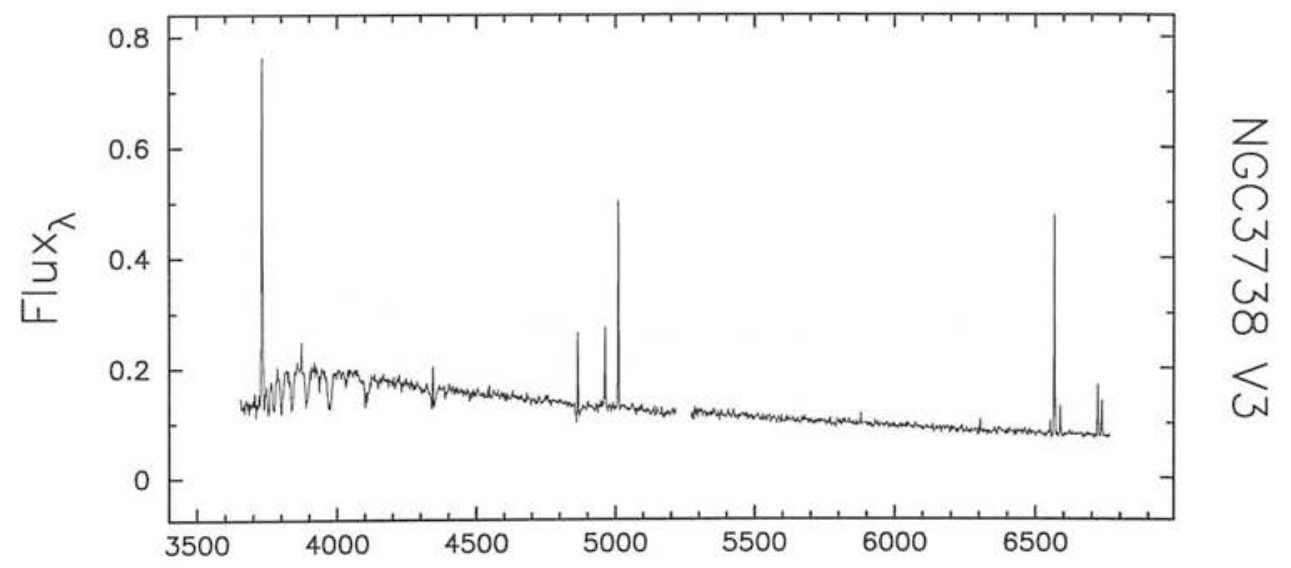

Angstroms

Figure 3.19: Flux in $10^{-15} \mathrm{ergs} \mathrm{s}^{-1} \mathrm{~cm}^{-2} \AA^{-1}$. 


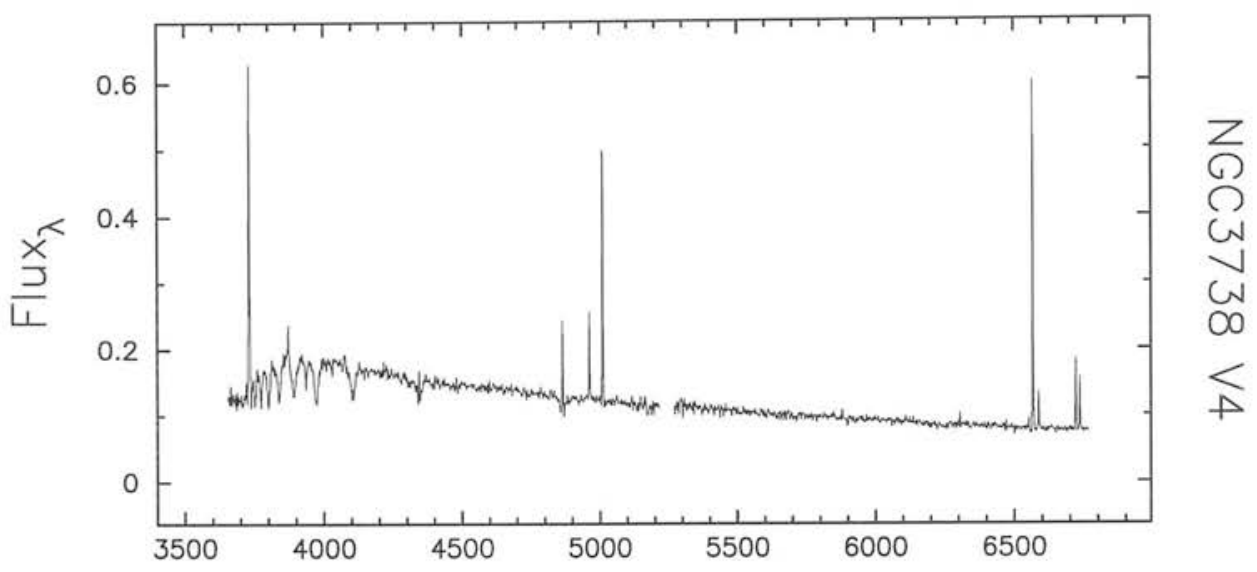

Angstroms

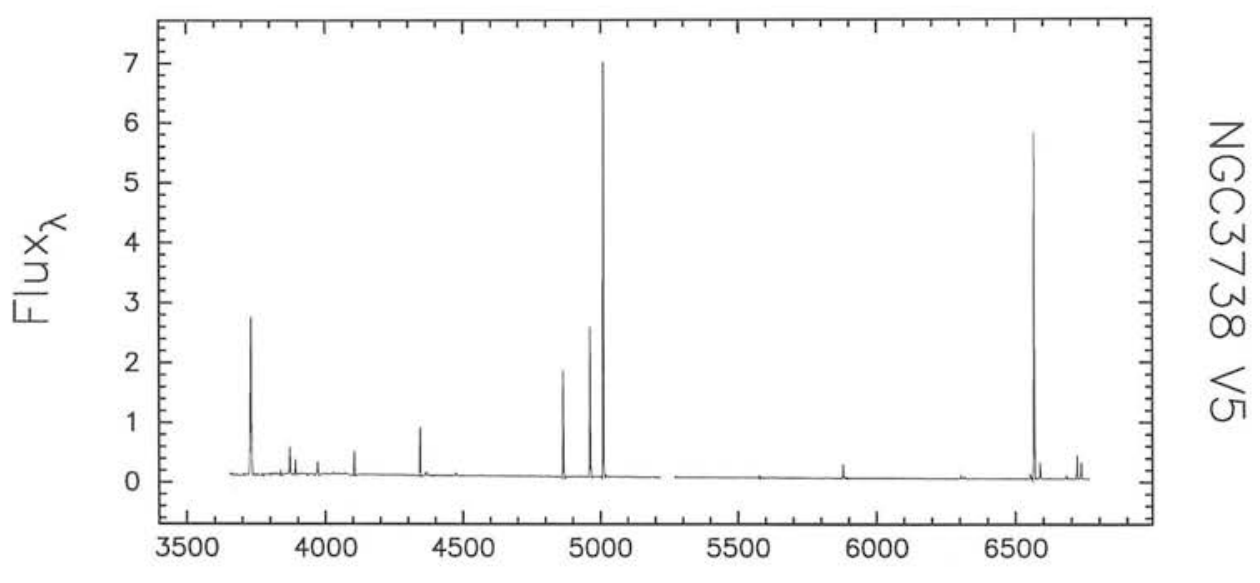

Angstroms

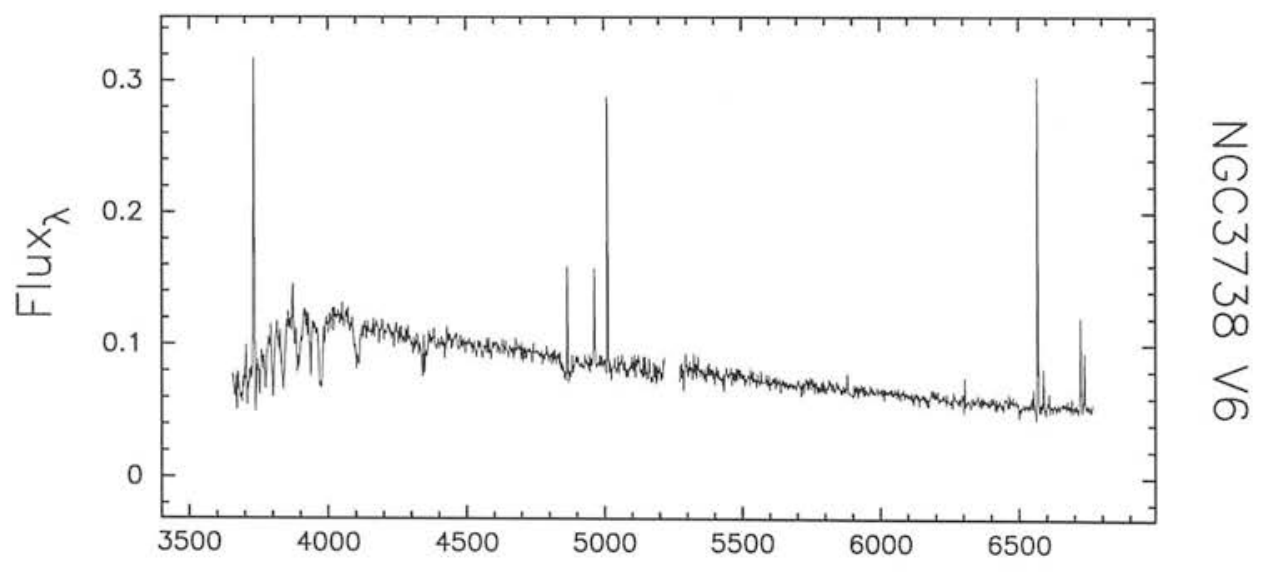

Angstroms

Figure 3.20: Flux in $10^{-15} \mathrm{ergs} \mathrm{s}^{-1} \mathrm{~cm}^{-2} \AA^{-1}$. 


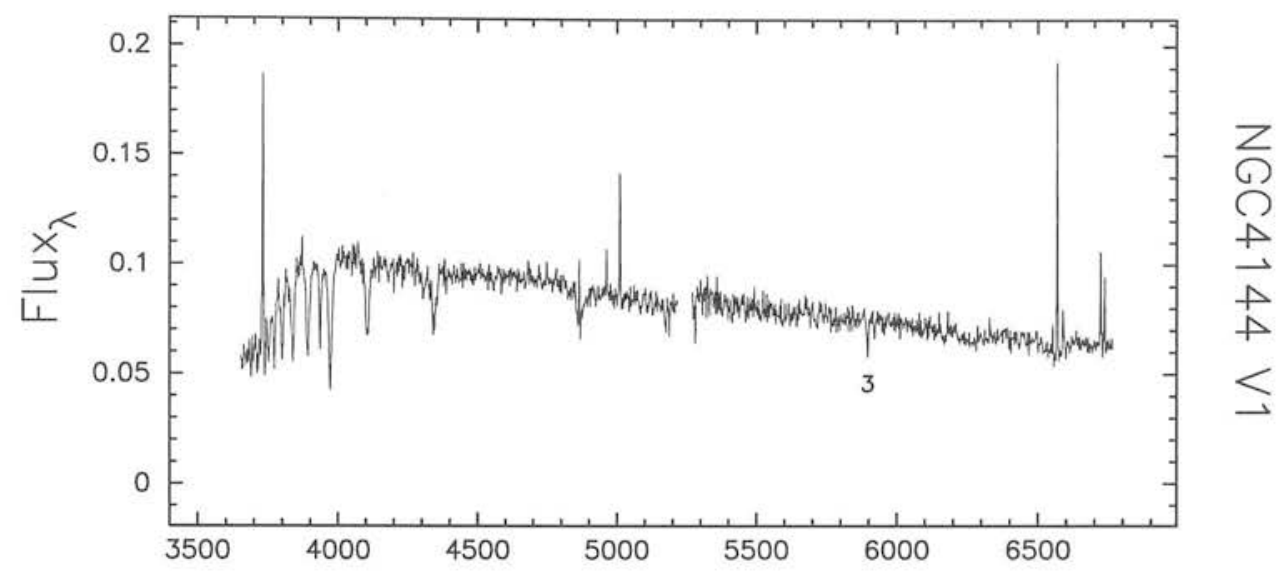

Angstroms

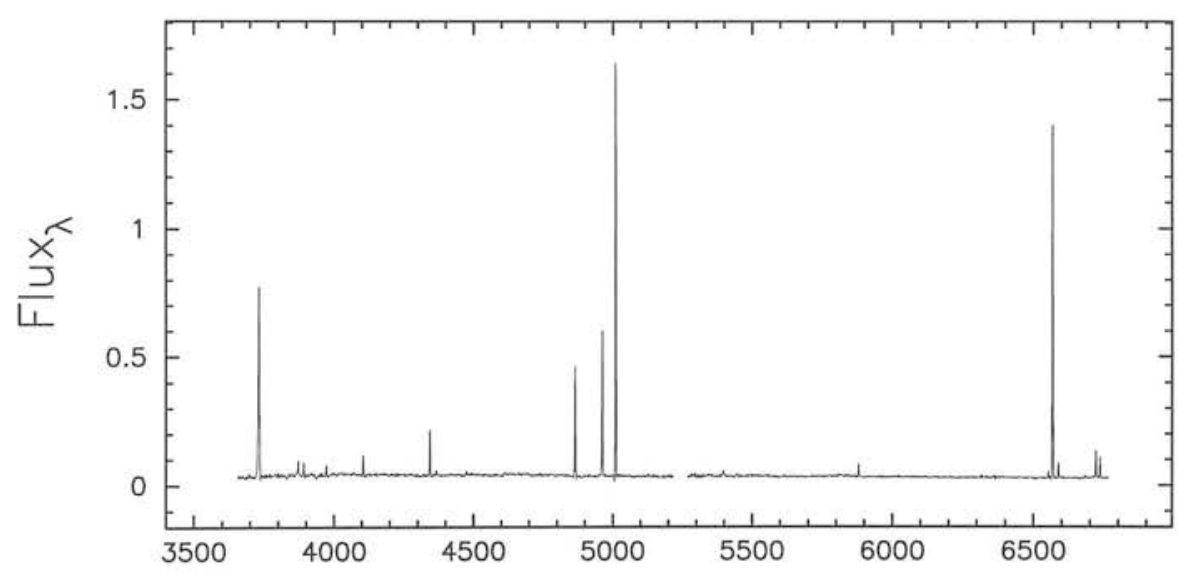

$Z$
$\Omega$
$\stackrel{A}{A}$
$\vec{A}$
$\stackrel{N}{N}$
$<$

Angstroms

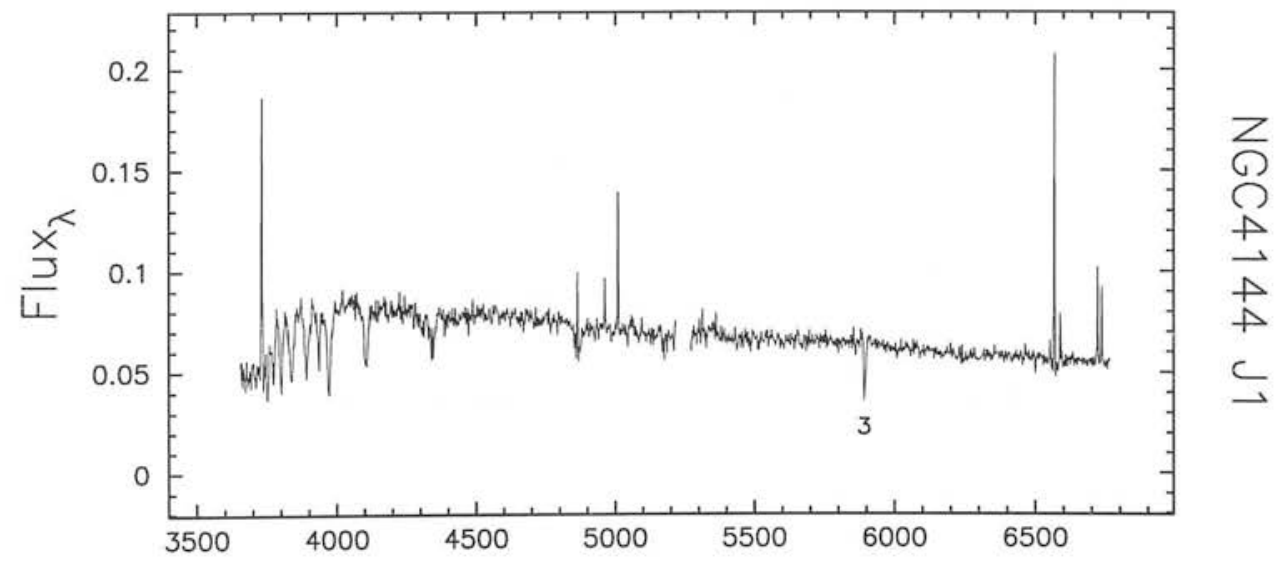

Angstroms

Figure 3.21: Flux in $10^{-15} \mathrm{ergs} \mathrm{s}^{-1} \mathrm{~cm}^{-2} \AA^{-1}$. 


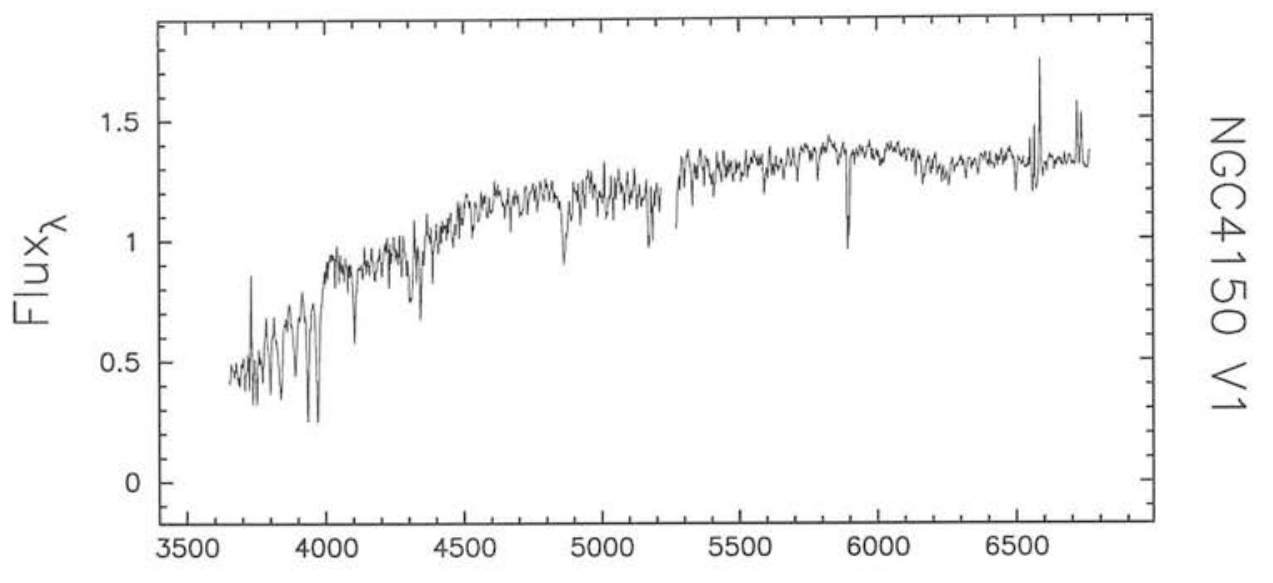

Angstroms

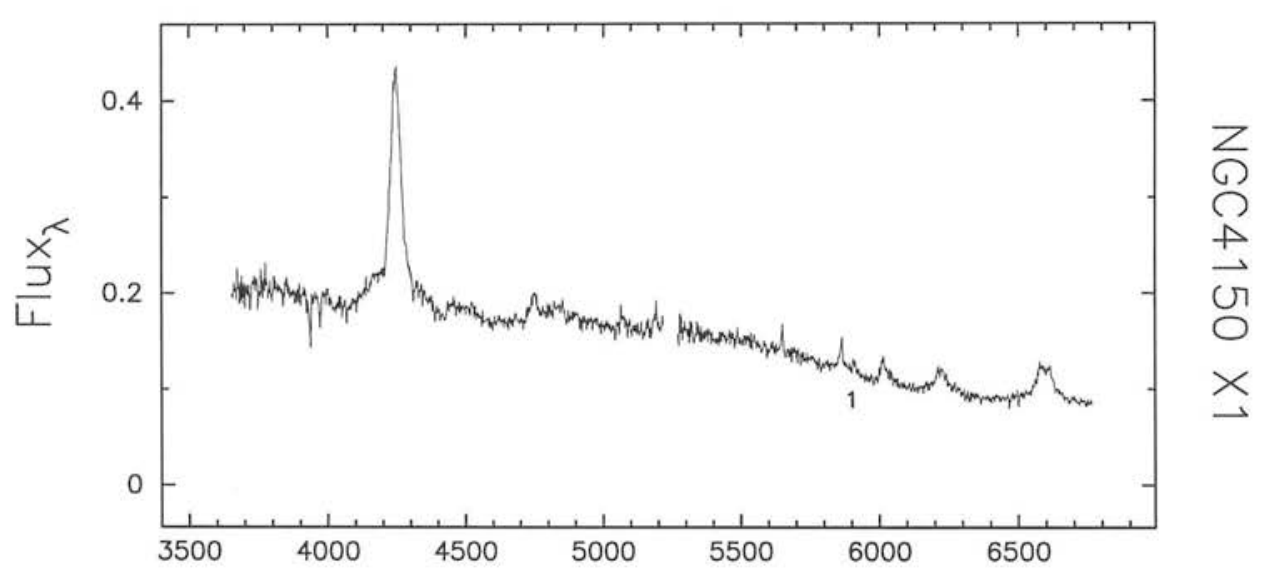

Angstroms

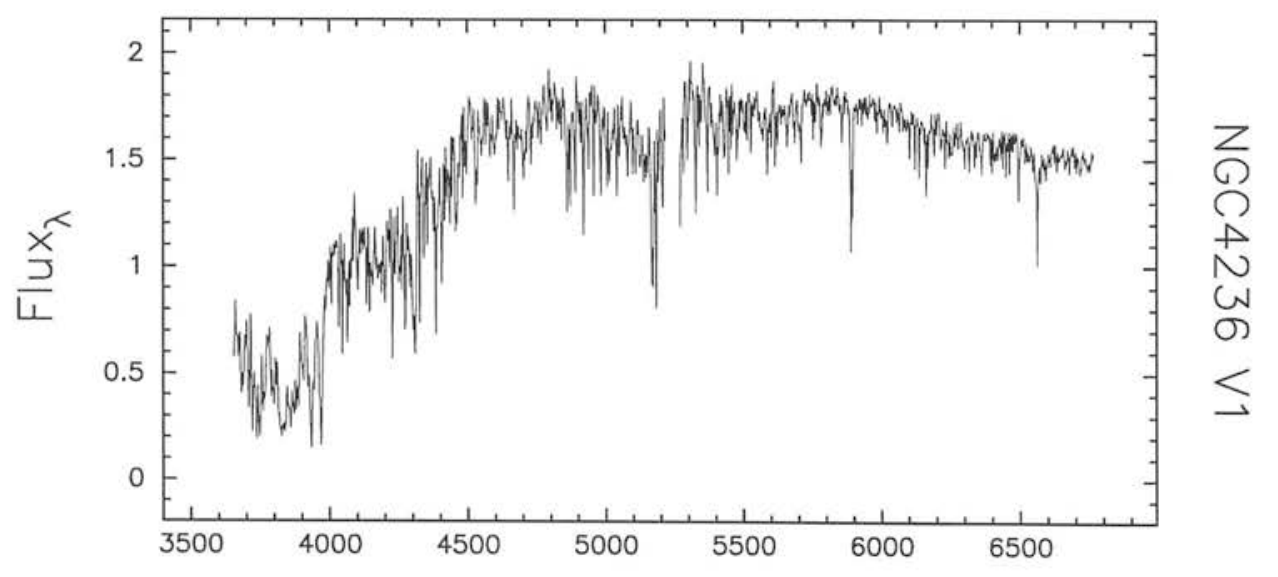

Angstroms

Figure 3.22: Flux in $10^{-15} \mathrm{ergs} \mathrm{s}^{-1} \mathrm{~cm}^{-2} \AA^{-1}$. 


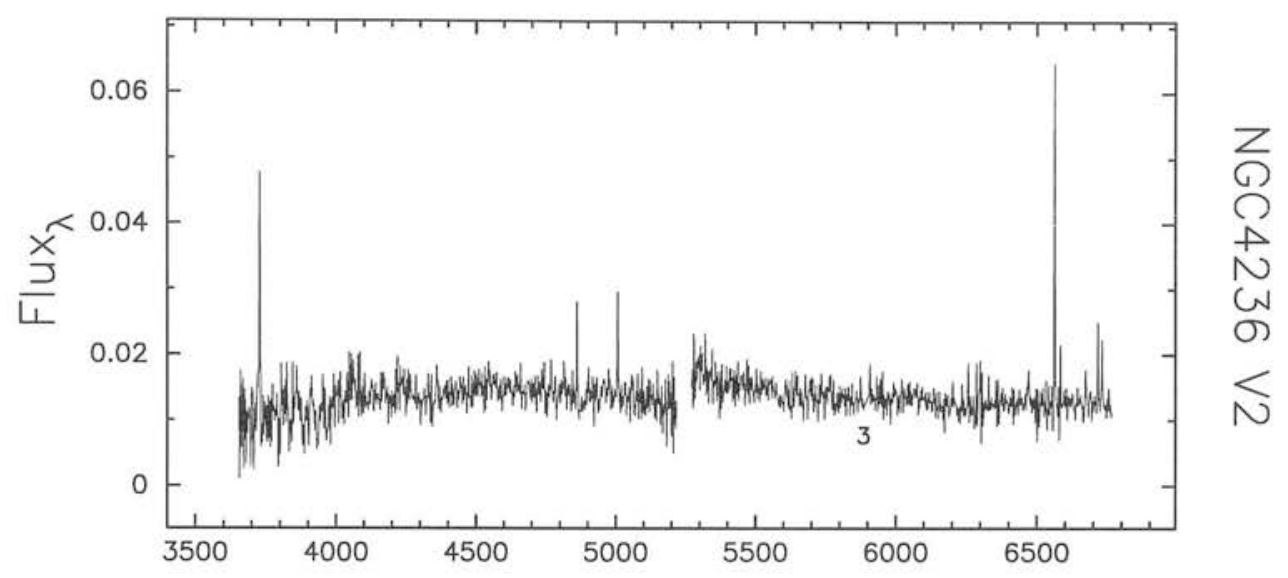

Angstroms

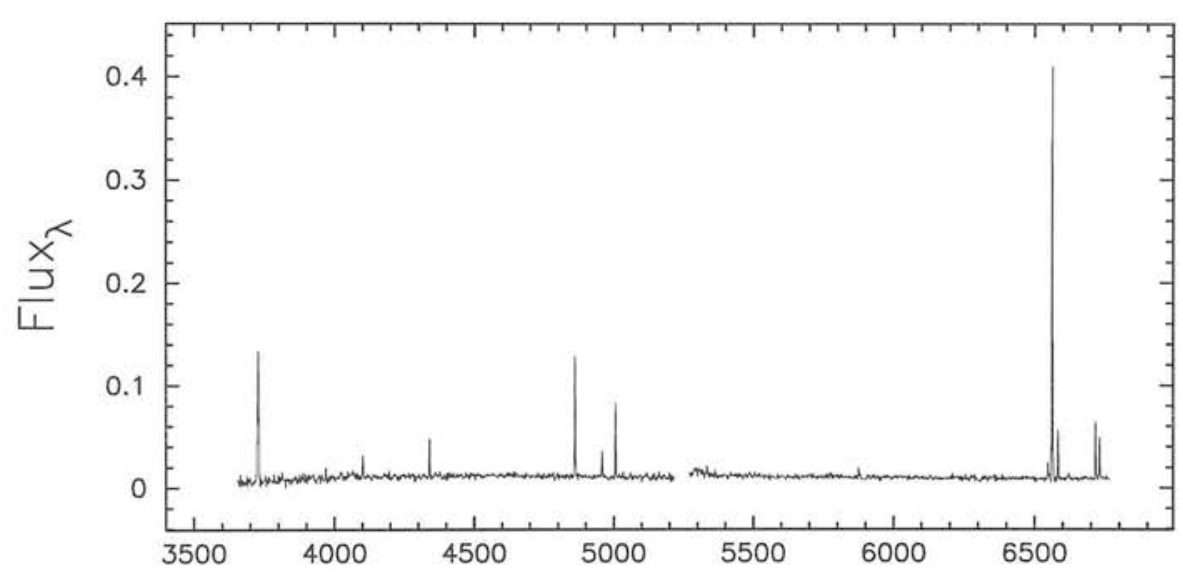

$Z$
$\Omega$
$\Omega$
$N$
$w$
a
$\vdots$

Angstroms

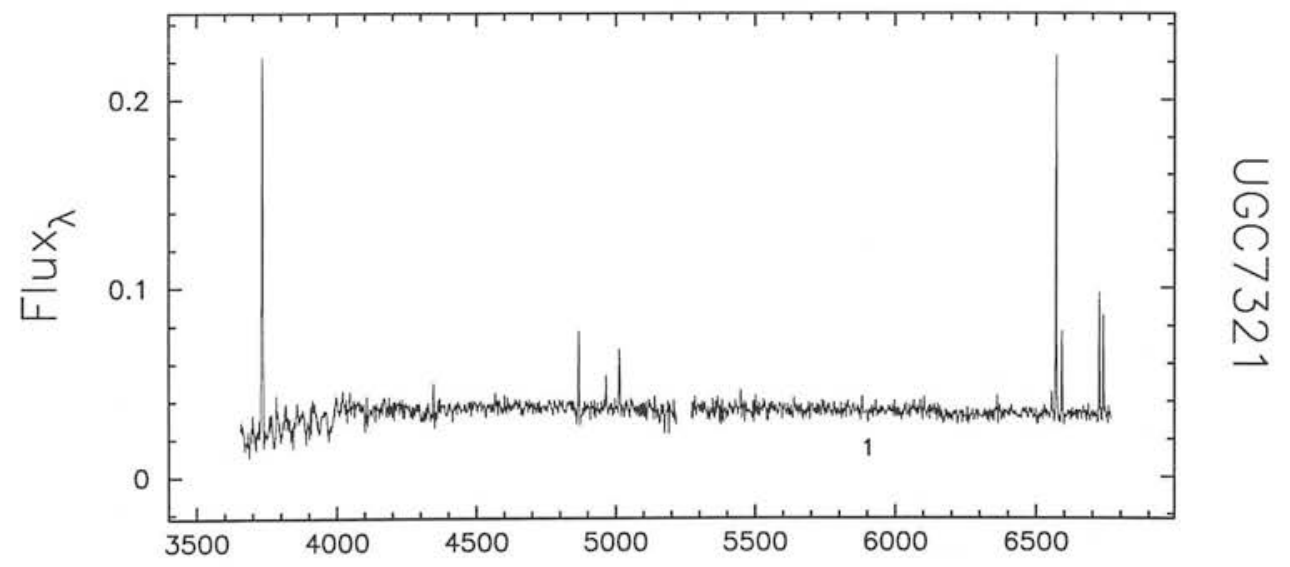

Angstroms

Figure 3.23: Flux in $10^{-15} \operatorname{ergs~s}^{-1} \mathrm{~cm}^{-2} \AA^{-1}$. 


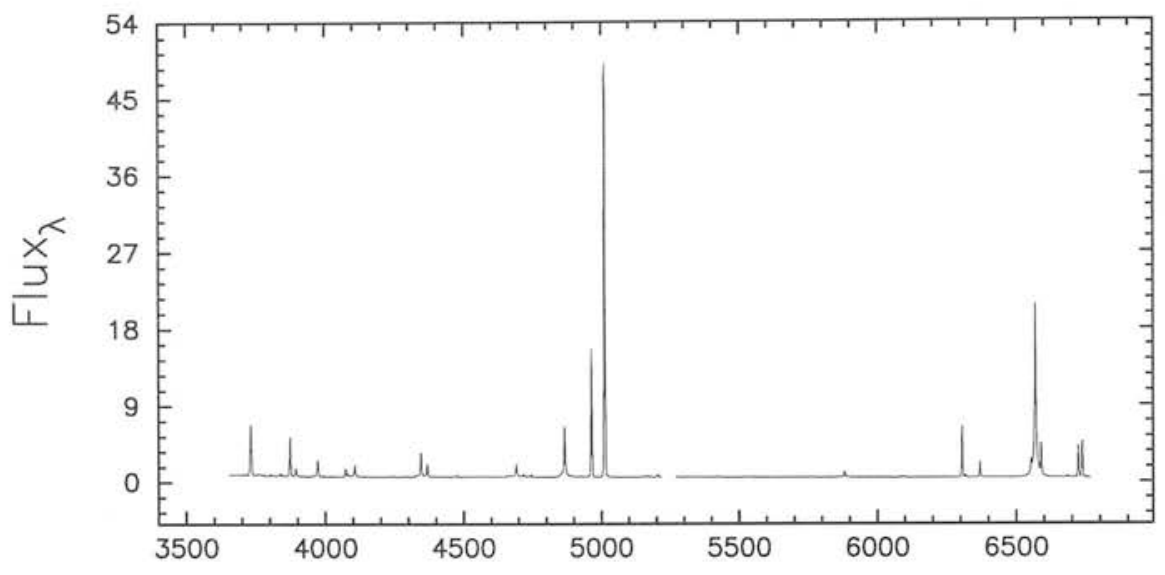

Angstroms

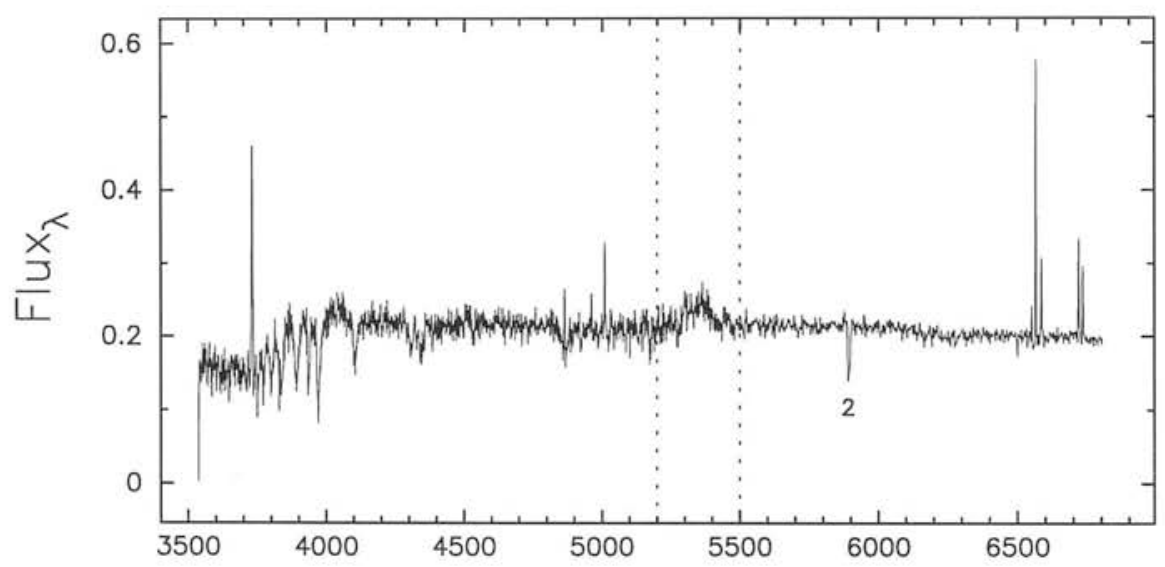

$z$
ก
a
음
or

Angstroms

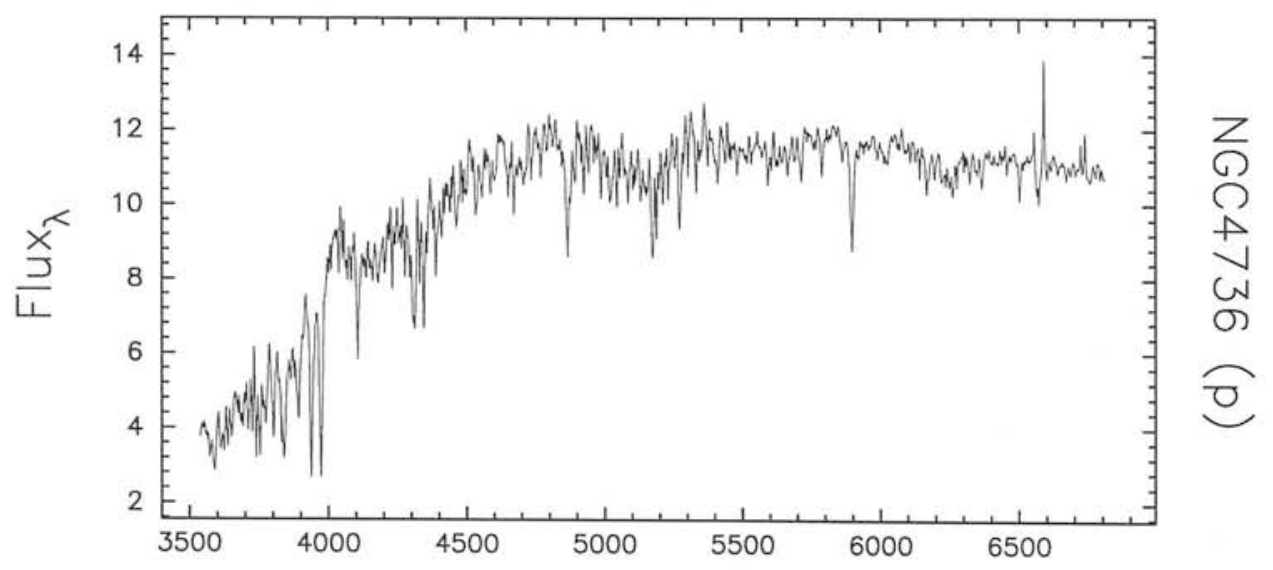

Angstroms

Figure 3.24: Flux in $10^{-15} \mathrm{ergs} \mathrm{s}^{-1} \mathrm{~cm}^{-2} \AA^{-1}$. 


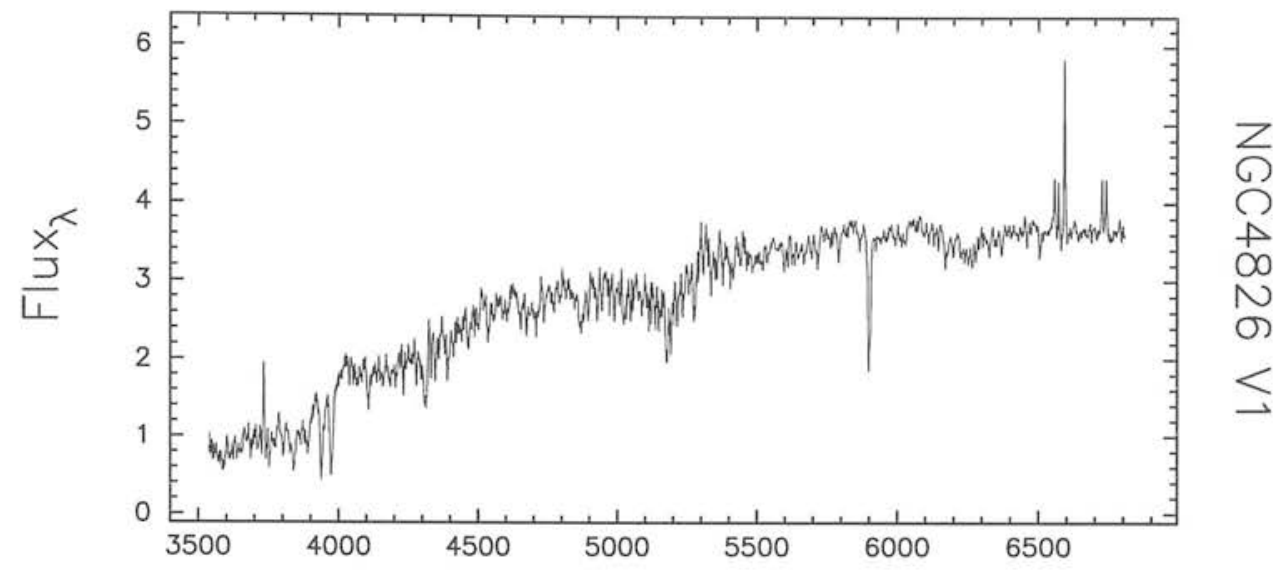

Angstroms

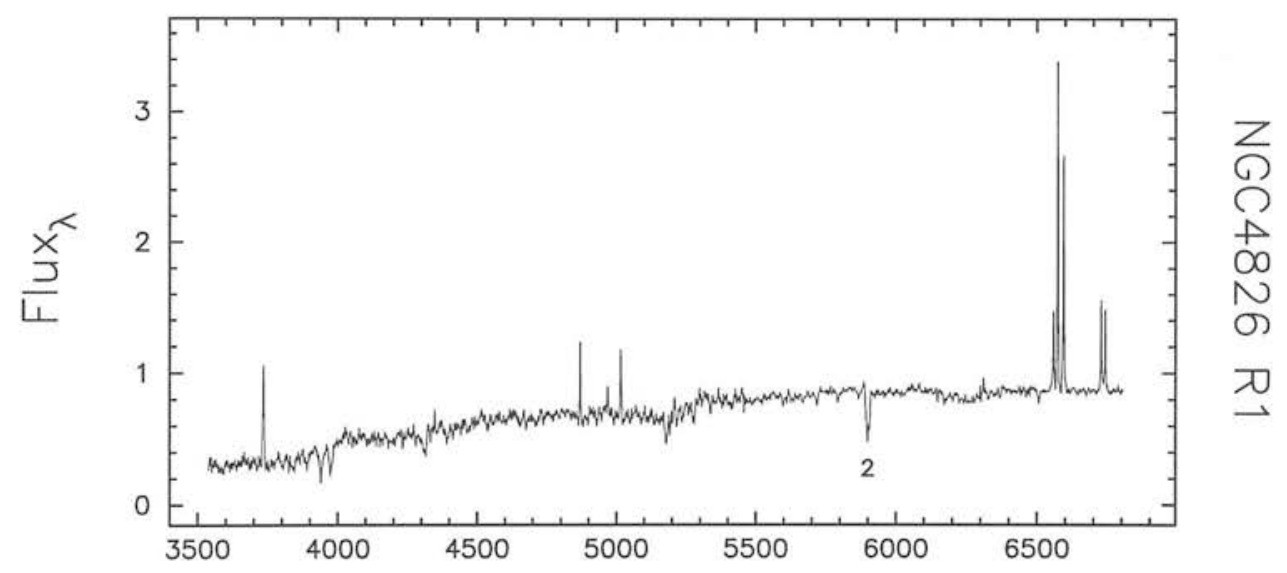

Angstroms

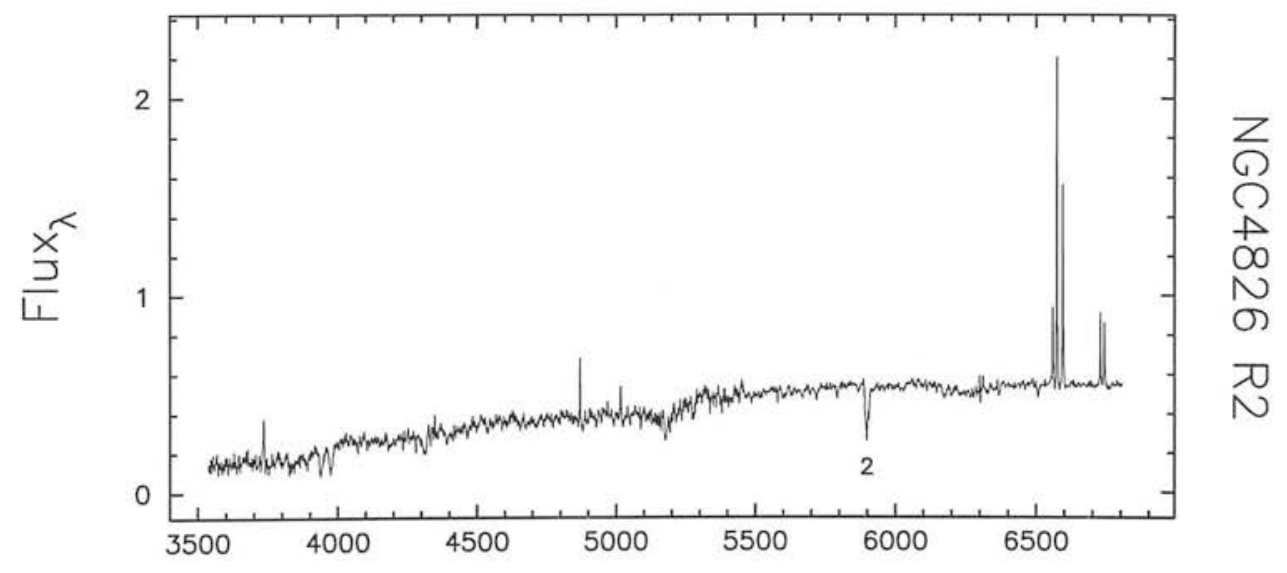

Angstroms

Figure 3.25: Flux in $10^{-15} \mathrm{ergs} \mathrm{s}^{-1} \mathrm{~cm}^{-2} \AA^{-1}$. 


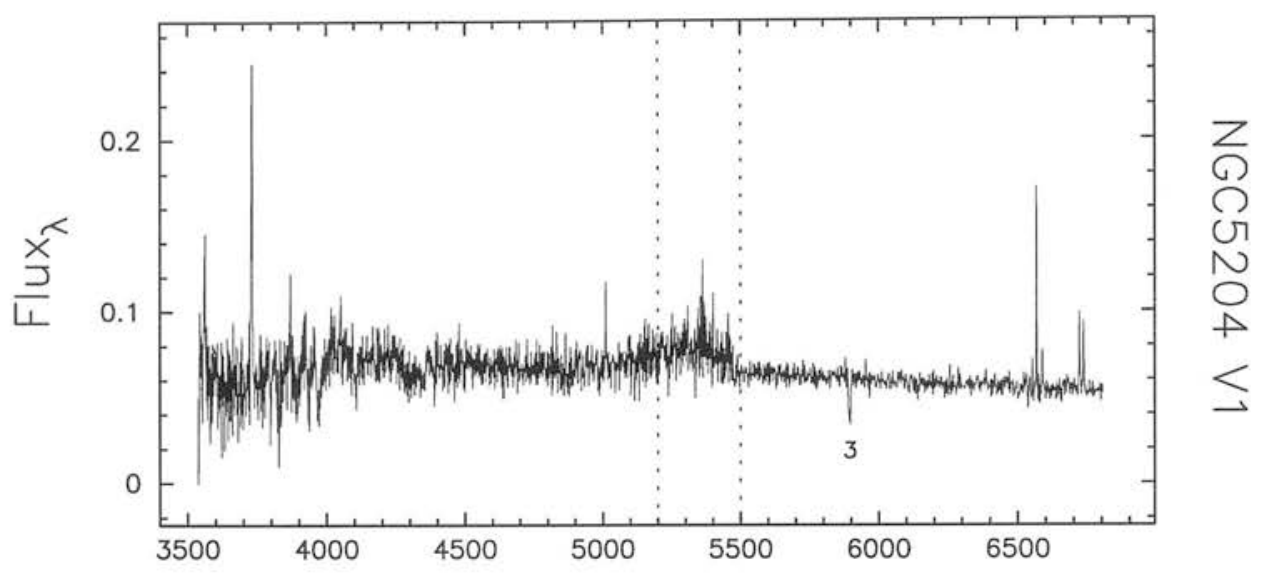

Angstroms

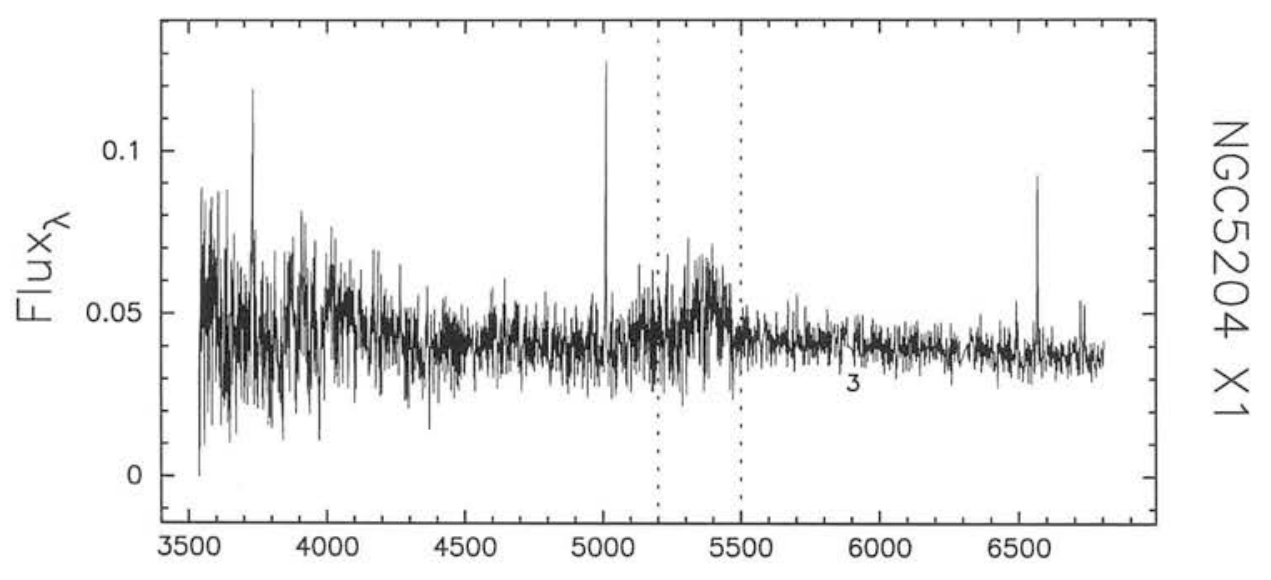

Angstroms

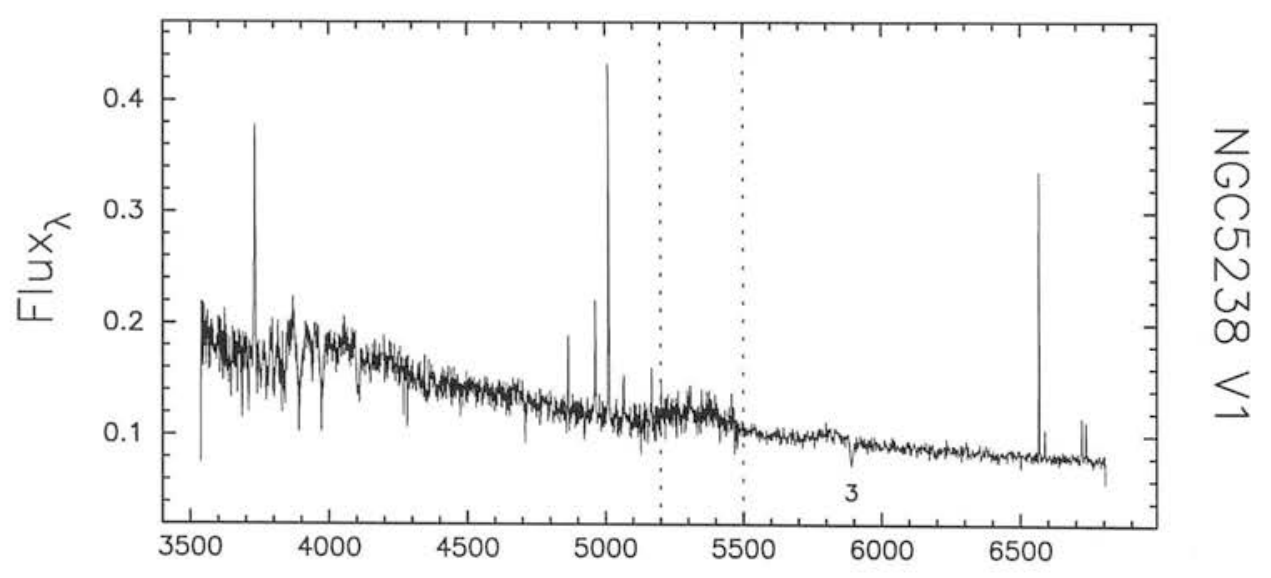

\section{Angstroms}

Figure 3.26: Flux in $10^{-15} \mathrm{ergs} \mathrm{s}^{-1} \mathrm{~cm}^{-2} \AA^{-1}$. 


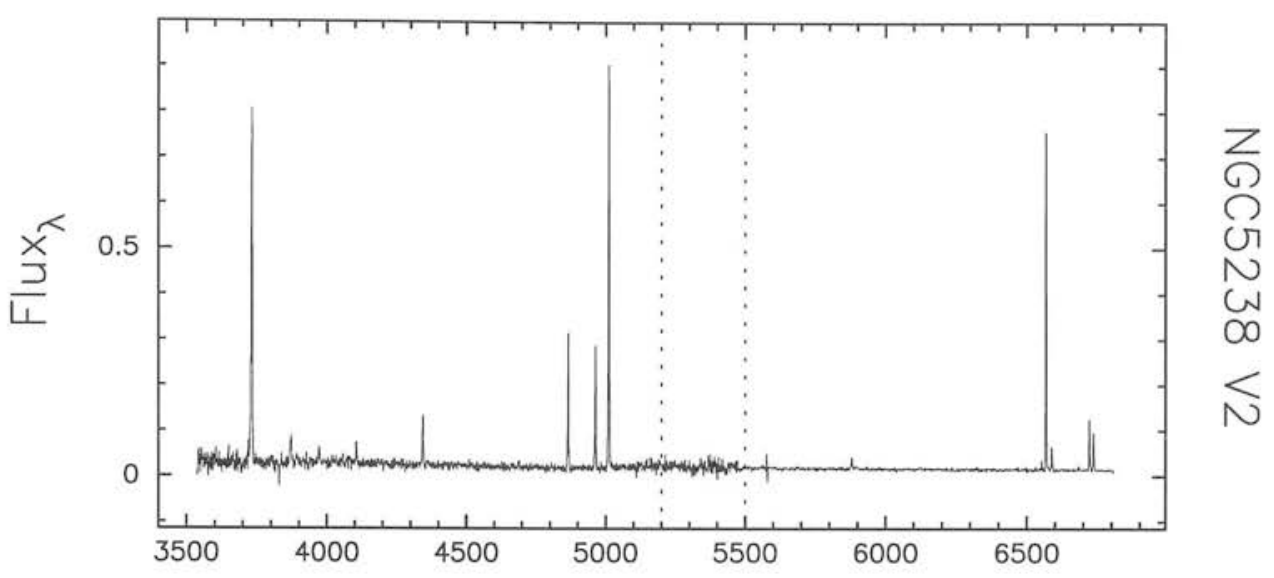

Angstroms

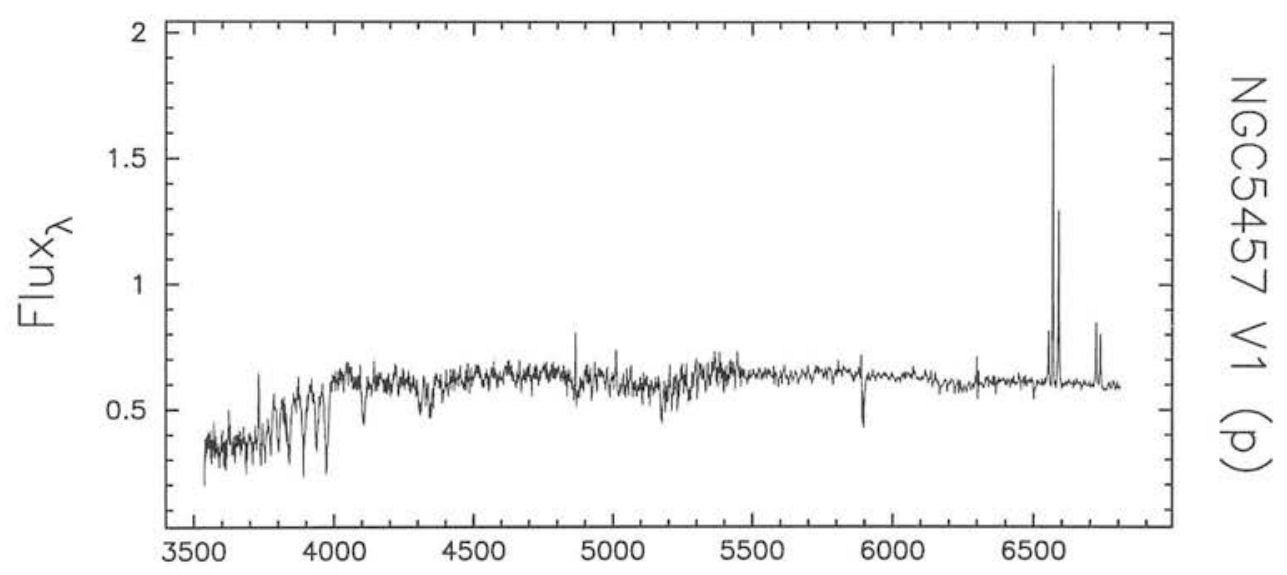

Angstroms

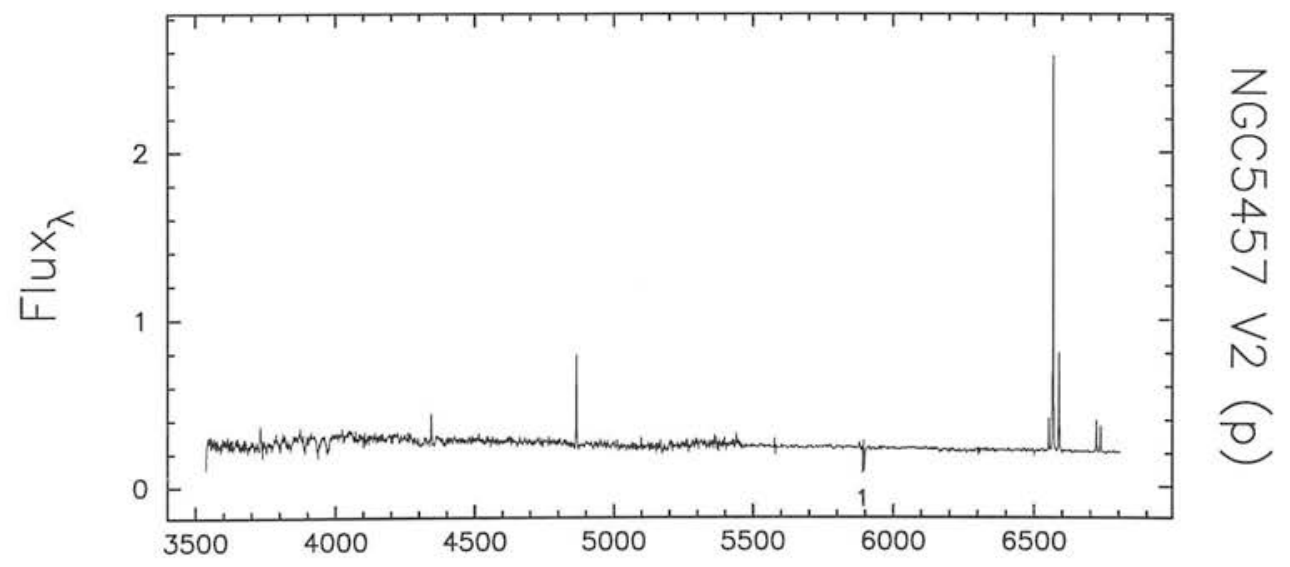

Angstroms

Figure 3.27: Flux in $10^{-15} \mathrm{ergs} \mathrm{s}^{-1} \mathrm{~cm}^{-2} \AA^{-1}$. 


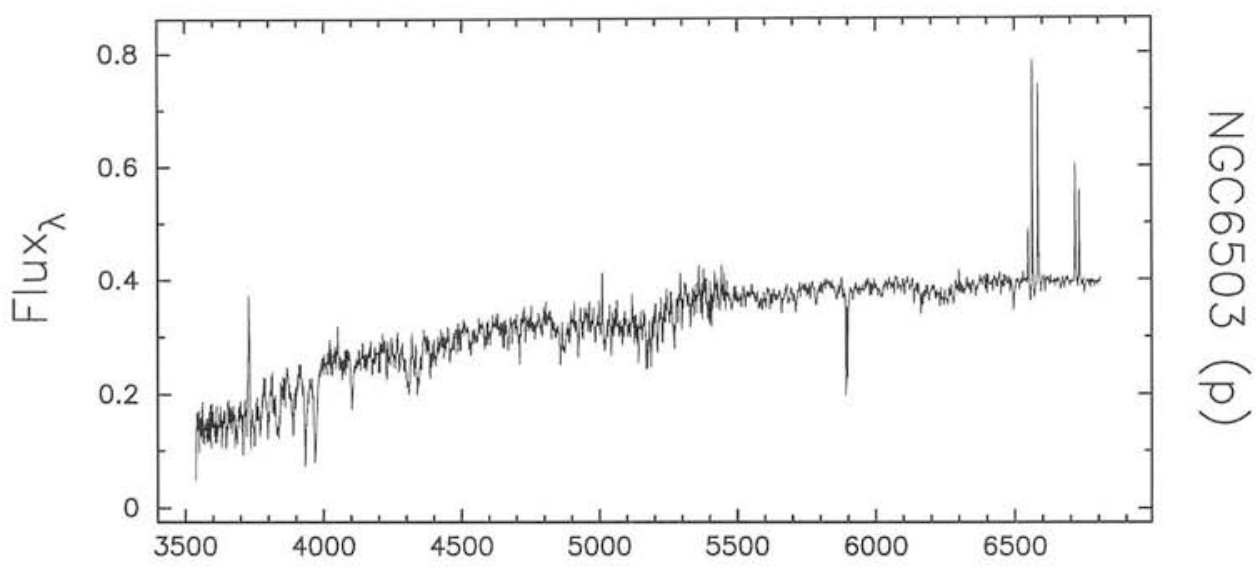

Angstroms

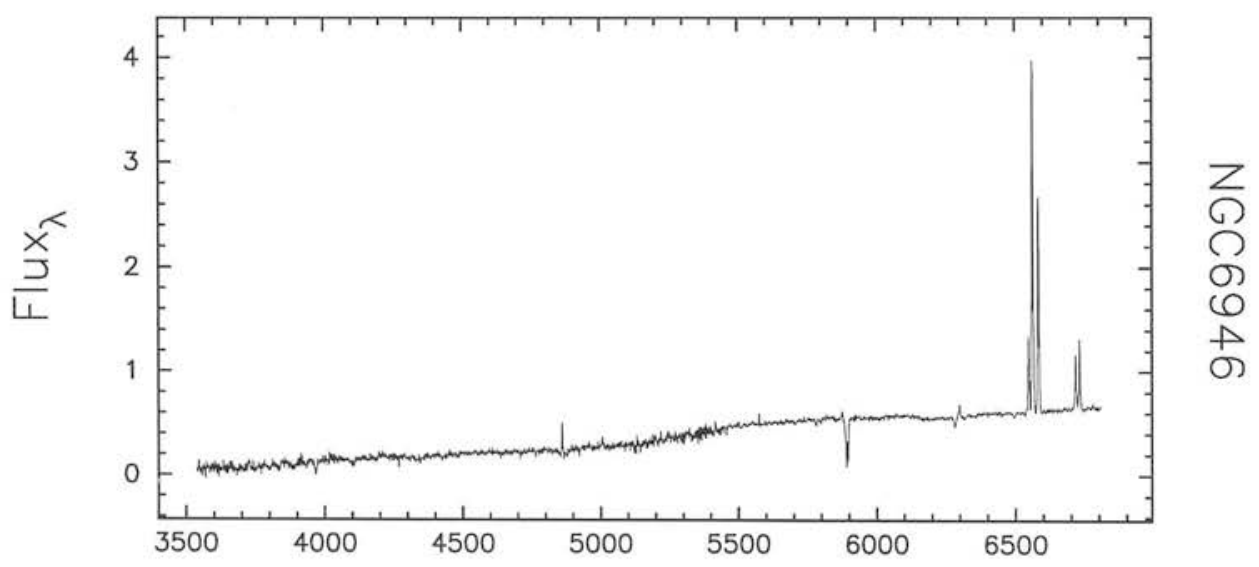

Angstroms

Figure 3.28: Flux in $10^{-15} \mathrm{ergs} \mathrm{s}^{-1} \mathrm{~cm}^{-2} \AA^{-1}$. 


\subsection{Notes on individual objects}

In what follows notes will be given for each galaxy. Whenever radio or X-ray targets were located in the nuclear region of the galaxies, it will be mentioned whether the targets were followed up with spectroscopic observations or not.

NGC 147: Three prominent optical sources are visible in the nuclear region of NGC 147 as can be seen in figure 3.5. It is not clear if any of these sources correspond to the nucleus of the galaxy. Sources V1 and V2 are unresolved, whereas the third source is resolved and probably corresponds to a globular cluster (Johnson 1997).

Observations for all 3 targets were acquired. The spectra of V1 and V2 can be seen in figure 3.8. The spectrum of the third source has such low $\mathrm{S} / \mathrm{N}$ that it is not shown here (although a hint of an absorption feature at $\mathrm{H} \alpha$ wavelength suggests the presence of an sizeable young star population).

Both V1 and V2 appear stellar and are probably foreground Galactic stars. Comparison with spectra from stellar libraries suggests that they are F-stars. V2 appears to be of a later type than V1, which is consistent with the broad band colours found by Johnson (1997). The NaD doublets in figure 3.8 seem too strong for an F-type which might suggests some problems with the subtraction of skylines.

Comparison with the spectral atlas of Ho, Filippenko \& Sargent (1995b) shows that their observation towards this galaxy was of V1.

NGC 185: In a blue image this galaxy has a fairly smooth surface brightness profile with two bright sources located in the nuclear region. The appearance changes dramatically towards longer wavelengths. In figure 3.5 a near-IR image shows how the galaxy breaks into several knots of emission in the J band.

Spectra were obtained for 4 of the knots seen in the J band. J1 and J3 correspond to the brightest sources seen at the blue band. The spectra show some weak emission lines such as $\mathrm{H} \alpha$, [NII] and [SII], and also absorption features such as higher order lines of the Balmer series and CaII $\mathrm{K}$ and $\mathrm{H}$ lines. Inspection of the spectrum published by Ho, Filippenko \& Sargent (1995b) suggests that their observation corresponds to J4. They classified this source as a Seyfert 2, although further analysis using the [OII] $\lambda 3727$ emission line, which is not available from the Ho, Filippenko \& Sargent (1995b) data, shows that in fact the knots in the nuclear region of this galaxy probably belong to the LINER class (see chapter 
5).

NGC 205: This galaxy has a very well defined nucleus. Its spectrum is extremely similar to the stellar foreground sources seen in NGC147. In this case, however, it corresponds to a nuclear population dominated by a relatively young population of stars.

The H $\alpha$ absorption profile shows a wide, shallow component and a sharp central core. The lack of emission lines suggests that this galaxy has lost most of its gas, probably due the close presence of $\mathrm{NGC} 224$, of which NGC 205 is a satellite, or to the absence of OB stars in the nucleus.

The nuclear spectrum is also shown by Ho, Filippenko \& Sargent (1995b).

NGC 221: This elliptical galaxy is also a companion to NGC 224. It has a very smooth surface brightness profile and an unresolved nucleus at the diffraction limit of the HST Planetary Camera (Lauer et al. 1992).

The nuclear spectrum shows weak Balmer absorption lines as well as a significant 4000 $\AA$ break and CaII $\mathrm{K}$ and $\mathrm{H}$ absorption features, implying that the nucleus is dominated by a relatively old population. In fact, stellar spectral synthesis techniques show that the population is more than 10 Gyr old with solar metallicities (Bica, Alloin \& Schmidt 1990).

As mentioned in chapter 2, dynamical studies of this galaxy have revealed the presence of a central dark massive object with mass $3 \times 10^{6} M_{\odot}$. The lack of detected AGN-like emission lines, X-ray or radio emission suggests that this central dark massive object is a dormant BH.

Since the off-nuclear X-ray source observed in this galaxy (see figure 2.8 in chapter 2) did not have an optical or radio counterpart it was not followed up with spectroscopic observations.

NGC 224: The nucleus of NGC 224 is also dominated by stellar absorption features. HST Planetary Camera observations show a double nucleus with the two components $0.49^{\prime \prime}$ apart (Lauer et al. 1993). One of these components is consistent with the position of the geometrical centre of the bulge, with a radio source (Crane, Dickel \& Cowan 1992), and, within the spatial resolution of the ROSAT and Einstein HRI, with a weak X-ray source (Trinchieri \& Fabbiano 1991; Primini, Forman \& Jones 1993). The spectrum shown in figure 3.10 was obtained with a seeing above $1^{\prime \prime}$ and therefore the light from both components of the double nucleus was integrated during the observations. 
NGC 247: The bright nucleus of this galaxy appeared unresolved in the JKT broad band images (Johnson 1997). The spectrum shows some weak emission lines and significant Balmer absorption features, implying a young nuclear population and the presence of $\mathrm{OB}$ stars. The off-nuclear X-ray sources X1 and X2 seen in figure 2.9 were not followed up with spectroscopic observations.

NGC 404: Figure 2.10 in chapter 2 shows that the nucleus of NGC 404 is coincident with an X-ray source $\left(L_{X}=1.7 \times 10^{38} \mathrm{ergs} \mathrm{s}^{-1}\right)$. Ho, Filippenko \& Sargent (1995b) classified this nucleus as a LINER and this classification is confirmed here using the [OII] $\lambda 3727$ emission line (see figure 5.2 in chapter 5). HST observations at UV wavelengths of this galaxy show that its nucleus is dominated by stellar absorption features from massive young stars (Maoz et al. 1998). This might indicate a stellar origin for the emission spectrum seen at optical wavelengths.

NGC 598: This bulge-free galaxy has an unresolved nucleus surrounded by a faint fuzz (Nieto \& Auriere 1982). As mentioned in chapter 2, the nucleus is also a strong X-ray source.

The peculiar [NII] $\lambda \lambda 6548,6584-\mathrm{H} \alpha$ features seen in figure 3.11, with [NII] in emission and $\mathrm{H} \alpha$ in sharp, deep absorption, have been noticed by Rubin \& Ford (1986). The absorption spectrum has been classified as A7 (Nieto \& Auriere 1982), although comparison of figure 3.11 with spectra from stellar libraries suggests a later type.

Visual inspection of the spectrum published by Ho, Filippenko \& Sargent (1995b) suggests that they might have acquired some other source, which shows a bluer continuum and obvious $\mathrm{H} \alpha$ emission, and not the nucleus of NGC 598 .

Maffei I: The extremely reddened spectrum of Maffei I is due to its location in the plane of the Galaxy. An extinction of $A_{V}=5.1 \pm 0.2$ magnitudes has been measured (Buta \& McCall 1983). The spectrum shows $\mathrm{H} \alpha$ in emission and several absorption features. Some hint of a complex or broad component can be seen in the $\mathrm{H} \alpha$ profile (FWZI $\sim 900 \mathrm{~km}$ $\mathrm{s}^{-1}$ ), although this is very difficult to verify without proper modelling of the underlying stellar emission (see figure 3.29). No lines are seen in the blue spectrum, probably because of the poor signal-to-noise ratio.

Maffei II: This galaxy has a starburst nucleus which was probably triggered by a recent interaction (Hurt 1994). Like Maffei I, Maffei II is located in the Galactic plane and therefore heavily obscured. The internal nuclear extinction has also been found to be high, with $A_{V} \gtrsim 10$ magnitudes (Hurt \& Turner 1991; Hurt et al. 1993), which explains the intricate 


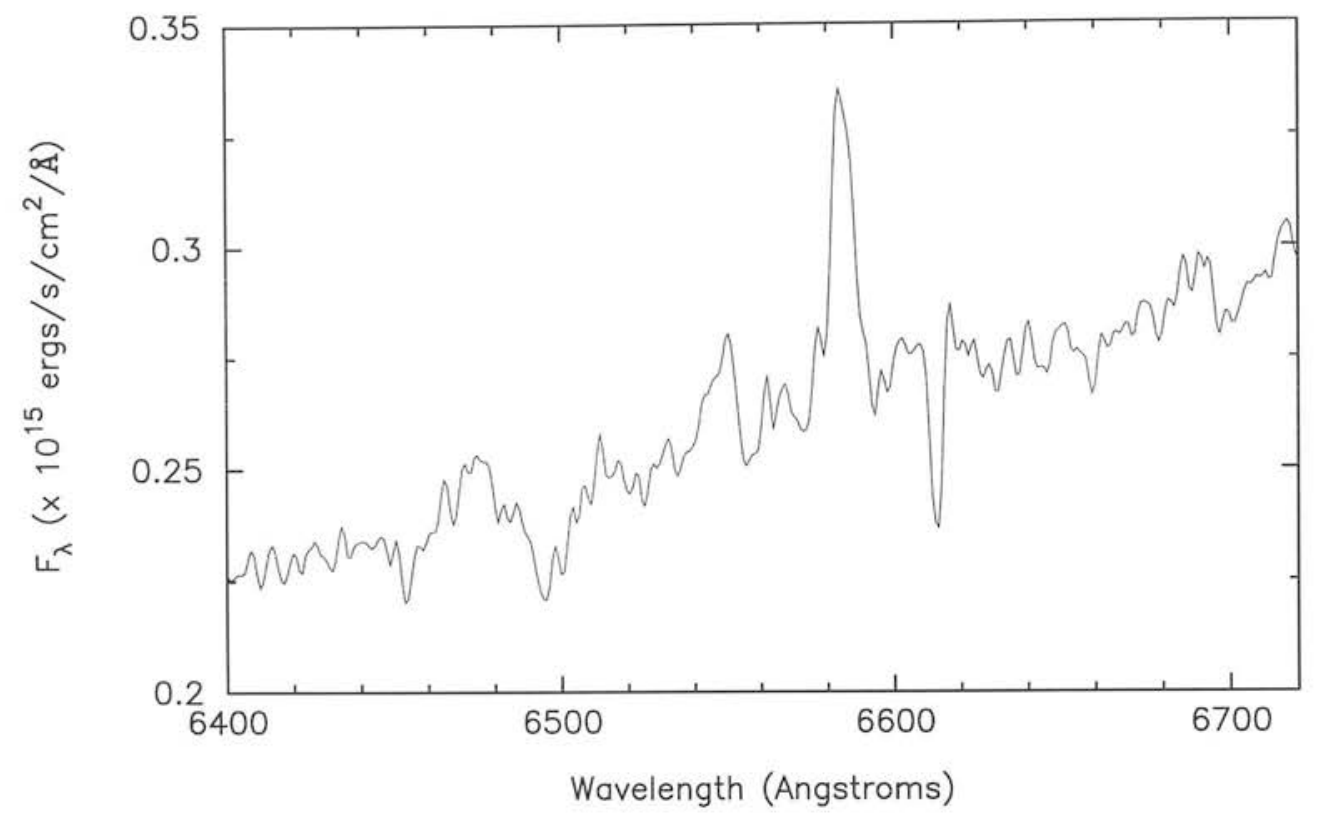

Figure 3.29: Detail of the Maffei I spectrum showing the $\mathrm{H} \alpha$ line emission.

morphology seen in figure 3.5. Near-infrared images show a much smoother distribution, with a peak somewhere between targets V1 and R1 (Johnson 1997). Nuclear thermal radio sources aligned in the north-south direction were observed by Turner \& Ho (1994). Two of them are coincident with peaks of optical emission. The spectra of these two sources and of the brightest optical knot are shown in figure 3.12 (the blue spectra had extremely low signal-to-noise ratio and are therefore not shown).

IC 342: An extended X-ray source is coincident with the starburst nucleus in IC 342 (see figure 2.11 in chapter 2). Infrared images show that the nucleus of the galaxy has a well defined peak, while optical images show an elongated morphology in the north-south direction (Johnson 1997). This elongation is due to two blue knots of emission located above and below the nucleus and $\sim 5^{\prime \prime}$ apart, with the southern component being the brightest.

Two targets were acquired in the nuclear region, the nucleus (V1) and the southern blue knot (V2 - see figure 3.5). Both spectra look extremely similar although the nucleus shows stronger absorption Balmer lines. Inspection of the data published by Ho, Filippenko \& Sargent (1995b) shows that their observations correspond to the galactic nucleus. No spectroscopic follow up was done for the off-nuclear X-ray sources X1 and X3 seen in figure 2.11 .

NGC 1560: This low luminosity galaxy harbours an HII nucleus. The low metallicity of the emitting gas can be appreciated from the small [NII] $\lambda 6584 / \mathrm{H} \alpha$ ratio. No signs of an 


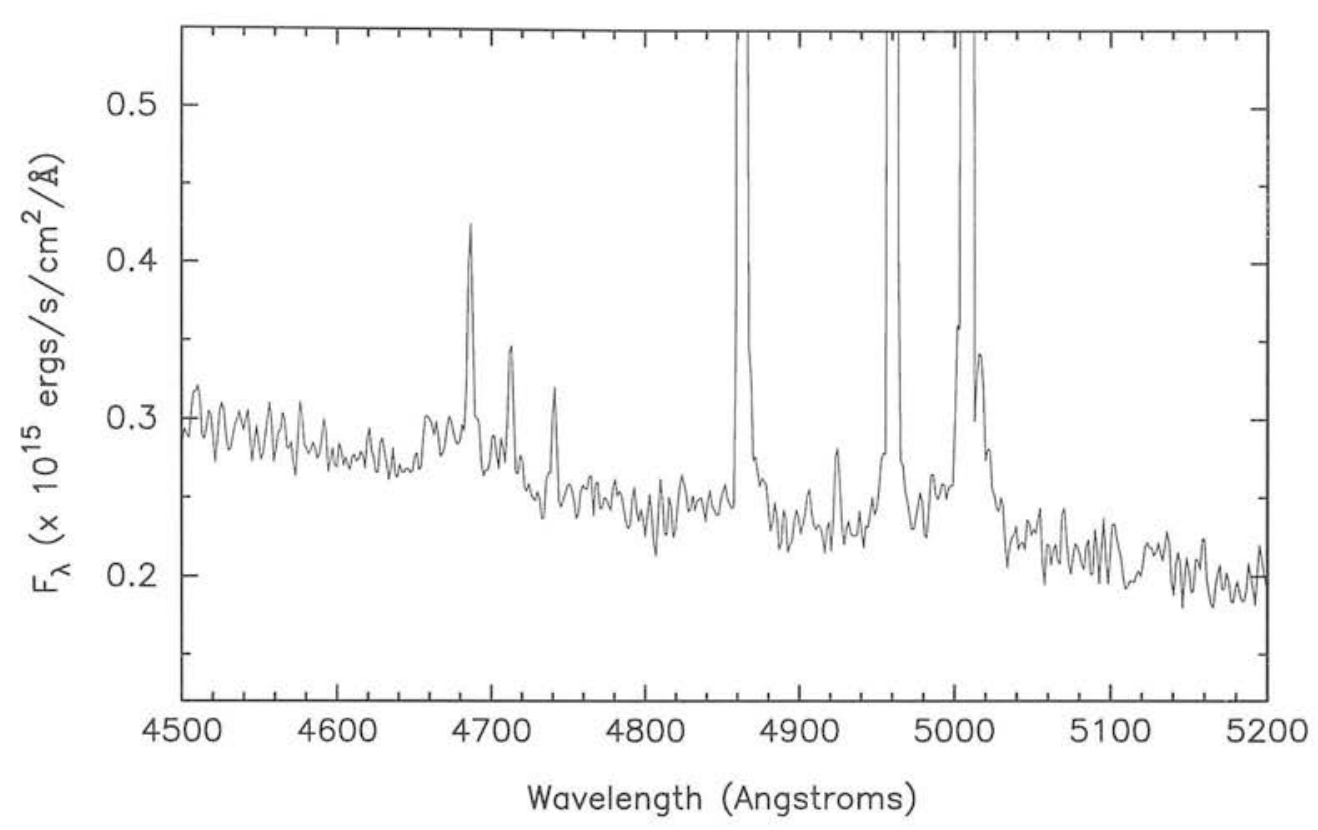

Figure 3.30: Detail of the NGC 2366 V4 spectrum showing broad components in the oxygen lines and a hump at $\sim 4660 \AA$ thought to be due to the presence of Wolf-Rayet stars.

underlying stellar population are seen in the spectrum in figure 3.14. The observed target corresponds to the same observations acquired by Ho, Filippenko \& Sargent (1995b).

NGC 2366: A good fraction of the body of this galaxy is shown in figure 3.5. Spectra were acquired for two bright sources located in the north and central part of the galaxy (V1 and V2) and for three sources located at or near the giant HII region NGC 2363 (V3, V4 and V5).

V1 shows some emission lines superimposed on the characteristic undulating spectrum of a late M-type star. This spectrum corresponds to the observations obtained by Ho, Filippenko \& Sargent (1995b). Johnson (1997) finds that the colours of this source are consistent with it being of M5-type.

$\mathrm{V} 2$ is an unresolved source and probably another foreground star of G-type.

The giant HII region NGC 2363 has been studied by Roy et al. (1992). They have found that V4 shows low intensity broad wings in $\mathrm{H} \alpha, \mathrm{H} \beta$ and [OIII] $\lambda .5007$ emission lines. Gonzalez-Delgado et al. (1994) also found broad emission lines at 4660 and $5810 \AA$ attributed to Wolf-Rayet stars. A detail of the spectrum of this source can be seen in figure 3.30. The small [NII] $\lambda 6584 / \mathrm{H} \alpha$ ratios observed in sources V3, V4 and V5 (figure 3.15) suggests low metallicity emitting regions.

NGC 2403: The nuclear region of this galaxy is dominated by two unresolved knots of 
emission (see figure 3.5). It is unclear if either of them represents the nucleus of the galaxy (Johnson 1997). Spectra for both sources were acquired and can be seen in figure 3.16. Both sources show absorption features and very weak nebular emission lines. V1 corresponds to the source observed by Ho, Filippenko \& Sargent (1995b). No spectroscopic follow up of the four off-nuclear X-ray sources seen in figure 2.14 was made.

NGC 2976: The B image in figure 3.5 shows two bright emission knots in the nuclear region of this galaxy. V1 is unresolved and is the only source visible in the $\mathrm{R}$ and infrared images. V2 becomes dominant in the $\mathrm{U}$ band. The spectra in figure 3.16 and 3.17 show strong emission lines and, for $\mathrm{V} 2$, the presence of a young underlying stellar population with strong blue continuum. V1 corresponds to the source observed by Ho, Filippenko \& Sargent (1995b). No spectroscopic follow up of the off-nuclear X-ray source seen in figure 2.15 was made.

A 0951+68: The data for this dwarf galaxy were obtained through INT service observations. No previous spectral observations have been published in the literature. A detailed analysis of the data has been reported by Johnson et al. (1997). The galaxy seems to have a double nucleus shown as V1 and V2 in figure 3.6. V2 corresponds to a high excitation low-metalicity star-forming region. Its spectrum is shown in figure 3.17. The region shows an extremely high value for [OIII] $\lambda 5007 / \mathrm{H} \beta$ and appears to have undergone a very recent star formation episode (Johnson et al. 1997).

UGC 6456: As was mentioned in section 2.4 the bright optical knots seen in figure 3.6 are displaced south from the centre of the outer envelope of the galaxy. Also shown in the figure is what seems to be the most probable optical counterpart of the X-ray source $\mathrm{X} 1$, but no spectroscopic data were acquired for this source. In figures 3.17 and 3.18 the spectra of four of the optical knots are shown.

The spectra of V1 and V2 look very similar, both showing a high [OIII] $\lambda 5007 / \mathrm{H} \beta$ ratio. In the V3 spectrum the ratio is much lower and in the V4 spectrum the [OIII] $\lambda 5007$ emission lines are completely absent.

The most interesting source is V4. Its spectrum shows very few emission lines, with a broad component in $\mathrm{H} \alpha\left(2300 \mathrm{~km} \mathrm{~s}^{-1}\right.$ FWZI - see figure 3.31) and $\mathrm{H} \beta$, as well as some weak absorption features at the blue end. The only observed oxygen line corresponds to $[\mathrm{OH}] \lambda 3727$. The lack of $[\mathrm{OIII}] \lambda 5007$ emission, when $\mathrm{H} \beta$ is observed, rules out the possibility of this source being an AGN (AGN are characterised by [OIII] $\lambda 5007 / \mathrm{H} \beta z 1$ ). Visual inspection of the spectrum reveals no hint of the broad emission lines attributed 


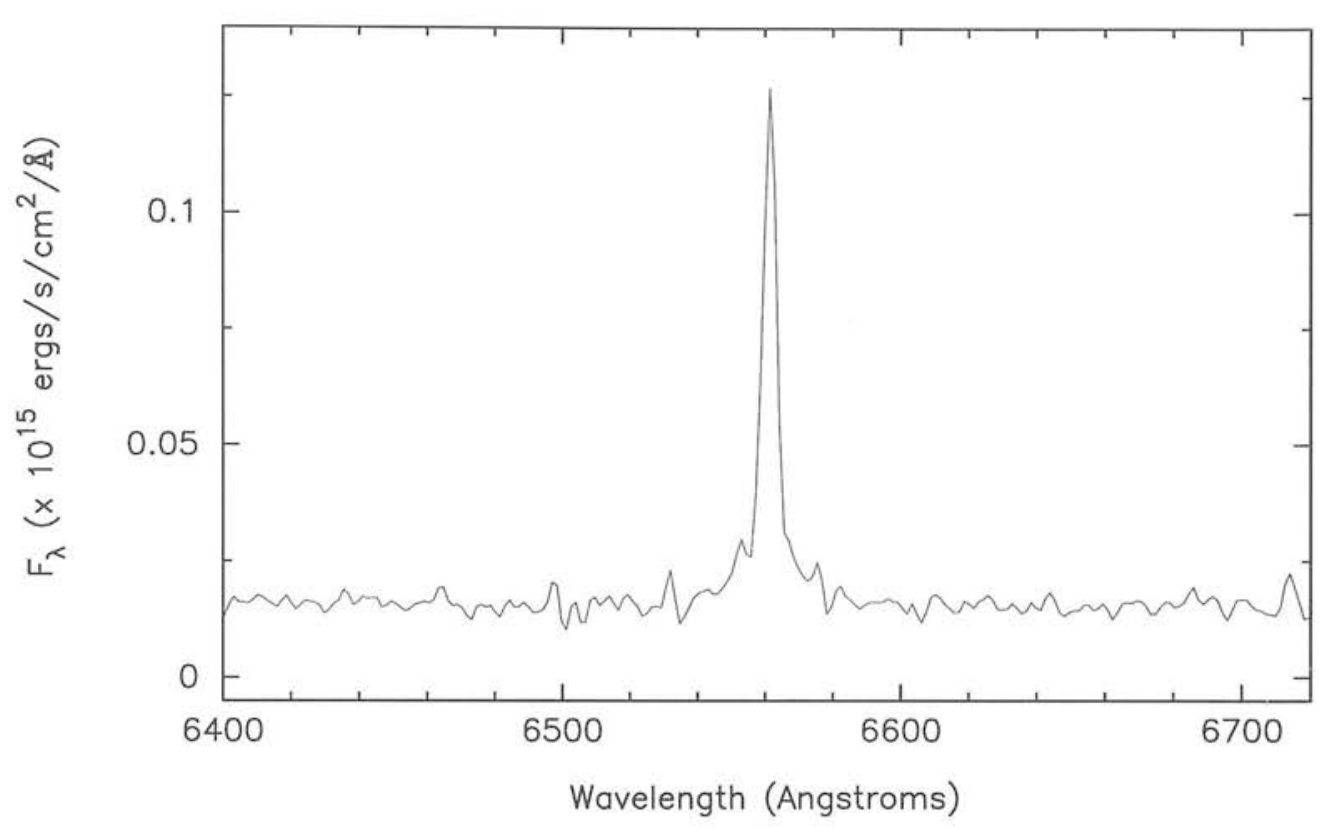

Figure 3.31: Detail of the UGC 6456 V4 spectrum showing the $\mathrm{H} \alpha$ broad component.

to Wolf-Rayet stars and the true nature of this source is a puzzle. It was not observed by Ho, Filippenko \& Sargent (1995b).

NGC 3738: Several knots of optical emission can be seen in the central region of this galaxy (figure 3.6), with the brightest sources being displaced to the north-west. The centre of ellipses fitted to the outer isophotes of the galaxy seen in infrared observations lies close the position of source V6 (Johnson 1997).

Spectra for 6 of these knots are shown here (figures 3.19 and 3.20). The spectra are a combination of pure HII regions with different contributions from an underlying young stellar population. Some beautiful examples of the Balmer series in absorption and emission can be seen in V3, V4 and V6.

All these sources are plotted in the [OI] $\lambda 6300 /[\mathrm{OIII}] \lambda 5007$ vs [OI] $\lambda 3727 /[\mathrm{OIII}] \lambda 5007$ diagnostic diagram shown in chapter 5 (empty circles in figure 5.2) and they all seem to have a [OI] $\lambda 6300 /[\mathrm{OIII}] \lambda 5007$ ratio slightly higher than normal HII regions.

Visual inspection of the spectrum published by Ho, Filippenko \& Sargent (1995b) seems to correspond to the observations of V1. The X-ray sources observed in figure 2.18 were not followed up with optical spectroscopy.

NGC 4144: The central region in this galaxy has a very ill defined morphology, as can be seen from figure 3.6 . 
The spectrum of source V1 in figure 3.21 corresponds to the brightest knot seen in the optical images. This seems to coincide with the object observed by Ho, Filippenko \& Sargent (1995b). The spectrum is a combination of nebular emission lines with significant absorption features from a young population of stars and significant blue continuum.

$\mathrm{J} 1$ corresponds to the brightest infrared peak and it is located $1.3^{\prime \prime}$ to the east of V1. Its spectrum is very similar to V1. V2 corresponds to a low metallicity HII region located $4^{\prime \prime}$ north from V1. The off-nuclear X-ray source X1 observed in figure 2.20 was not followed up with optical spectroscopy.

NGC 4150: The nucleus of this galaxy has been classified as a transition object by Ho, Filippenko \& Sargent (1997b). The spectrum, dominated by absorption features, also shows some weak emission lines. The $\mathrm{H} \alpha$ flux quoted in table 3.6 should be considered a lower limit, since no correction has been made to account for the absorption component.

$\mathrm{X} 1$ corresponds to a knot of emission $15^{\prime \prime}$ away from the nucleus and is consistent with the position of an X-ray source discussed in chapter 2 (see also figure 3.6). Its spectrum reveals that it is in fact a background quasar at redshift 0.52 . Its X-ray luminosity corresponds to $1.88 \times 10^{45} \mathrm{ergs} \mathrm{s}^{-1}$. The strongest line, seen at $4246 \AA$ in figure 3.22 , corresponds to $\operatorname{MgII} \lambda 2798$.

NGC 4236: This is a low surface brightness galaxy without an obvious nucleus. Spectra of 3 optical knots of emission were obtained (see figure 3.7 ).

V1 is unresolved in the JKT broad band images and its stellar spectrum is shown in figure 3.22. It probably corresponds to a Galactic foreground object. Comparison with spectra in stellar libraries suggests that it is a late G-type star. This is confirmed by the colours determined by Johnson (1997).

V2 shows strong emission lines and some evidence of absorption features from an underlying stellar population, while V3 has an HII region spectrum.

None of these sources corresponds to the observation published by Ho, Filippenko \& Sargent (1995b). Their spectrum shows an HII region with $\mathrm{H} \beta$ lower than [OII] $\lambda 5007$, while the spectra of both $\mathrm{V} 2$ and $\mathrm{V} 3$ have $\mathrm{H} \beta$ comparable to, if not larger than, [OIII] $\lambda 5007$.

The off-nuclear X-ray source X1 observed in figure 2.22 was not followed up with optical spectroscopy. 
UGC 7321: The position of the nucleus of this nearly edge-on galaxy was determined from a DSS image. The spectrum, seen in figure 3.23 shows strong emission lines and absorption from a young underlying stellar population. This galaxy was not observed by Ho, Filippenko \& Sargent (1995b).

NGC 4395: Two spectra were obtained of the dwarf Seyfert nucleus of this galaxy, one on July 1996 and the other on January 1997. They are analysed in detail in chapter 4. The off-nuclear X-ray source X2 observed in figure 2.24 was not followed up with optical spectroscopy.

NGC 4605: The nucleus of this galaxy is well defined in the infrared images and corresponds to the brightest knot of emission seen in the galaxy at optical wavelengths (Johnson 1997). The spectrum of the nucleus can be seen in figure 3.24 and is dominated by emission lines and absorption features from a young stellar population. It clearly corresponds to the source observed by Ho, Filippenko \& Sargent (1995b).

NGC 4736: The bright nucleus of this galaxy was classified as a. LINER by Heckman (1980) and is a strong X-ray source (see figure 2.26). The nuclear spectrum seen in figure 3.24 is dominated by absorption lines. For this reason, no Balmer line fluxes have been measured. Taniguchi et al. (1996) studied the weak emission lines using stellar population synthesis techniques and found that the nucleus shows marginal differences between a LINER and a HII region. Ho, Filippenko \& Sargent (1995b) also found a similar result, although they finally classified the nucleus as a pure LINER in Ho, Filippenko \& Sargent (1997b).

NGC 4826: The nucleus of this galaxy has been classified as a transition object by Ho, Filippenko \& Sargent (1997b). The galaxy has an unusual morphology because of a prominent dust lane seen across the disk. Nine compact radio sources were detected by Turner \& Ho (1994) - they are all located within $19^{\prime \prime}$ of the galactic centre and one of them is coincident with the nucleus.

Spectra were acquired for the nucleus (V1) and for two radio sources located $3^{\prime \prime}$ west (R1) and $4^{\prime \prime}$ north (R2) of the nucleus (see figure 3.7). The spectra can be seen in figure 3.25. They are dominated by emission lines, except for V1 where many absorption features are seen. The $\mathrm{V} 1 \mathrm{H} \alpha$ flux quoted in table 3.6 should be considered a lower limit, since no correction has been made to account for the absorption component.

NGC 5204: The $\mathrm{R}$ image in figure 3.7 shows the central region of this galaxy. V1 corresponds to the brightest knot seen in the infrared images, although it is not dominant 
in the $\mathrm{B}$ and $\mathrm{U}$ bands. It is also close to the centre of symmetry of the galaxy (Johnson 1997). The spectrum of this source can be seen in figure 3.26 and it seems to correspond to the observations reported by Ho, Filippenko \& Sargent (1995b). It is dominated by emission lines and some absorption features can also be seen in the low signal-to-noise ratio blue end.

$\mathrm{X} 1$ corresponds to the closest optical counterpart of the bright X-ray source seen in figure 2.28. A spectrum of this source is also shown in figure 3.26. Despite the low signal-tonoise ratio of the data it is possible to appreciate several strong emission lines denoting the presence of a star forming region.

NGC 5238: This galaxy has a bright unresolved knot of emission in its central region. However, it is displaced from the centre of symmetry of the galaxy (Johnson 1997). Spectra of this source (V1) and of another dimmer optical knot located $\sim 4^{\prime \prime}$ north from V1 were obtained (see figure 3.7). They can be seen in figures 3.26 and 3.27 . V1 shows strong emission lines, evidence for an underlying star population, and a very blue continuum. V2 obviously corresponds to an HII region. This galaxy was not observed by Ho, Filippenko \& Sargent (1995b).

NGC 5457: Two sources were observed in the central region of this galaxy. V1 corresponds to the nucleus, which is the brightest knot of emission seen in figure 3.6 and is also an X-ray source. Its position is coincident with the peak observed in infrared images (Johnson 1997). This source corresponds to the observations reported by Ho, Filippenko \& Sargent (1995b). V2 is a knot located just south of the nucleus.

The spectra of both sources (figure 3.27) are dominated by strong emission lines. Absorption features can be seen at the blue end, particularly for the nucleus.

None of the off-nuclear X-ray sources seen in figure 2.31 were observed with optical spectroscopy.

NGC 6503: The nucleus of this galaxy has been classified by Ho, Filippenko \& Sargent (1995b) as a transition-object-Seyfert-2 nucleus. Its spectrum shows strong emission lines and a red continuum. Inspection of the data in figure 3.28 reveals that a correction should be applied to the $\mathrm{H} \alpha$ flux quoted in table 3.6 since an absorption component is present. Although Ho, Filippenko \& Sargent (1995b) obtained a spectrum of the nucleus of the galaxy, it only corresponds to the red arm (i.e., in the $\sim 6210-6860 \mathrm{~A}$ range). A possible extended nuclear X-ray source in this galaxy was discussed in chapter 2. 
NGC 6946: The nucleus of this galaxy is a strong X-ray emitter (see figure 2.33). The strong emission lines in the nuclear spectrum can be appreciated in figure 3.28 . The steep continuum and the large Balmer decrement denotes the strong extinction that affects this galaxy (see tables 3.6 and 5.1). The nucleus was also observed by Ho, Filippenko \& Sargent (1995b). None of the numerous off-nuclear X-ray sources seen in figure 2.33 were followed up with optical spectroscopy. 


\section{Chapter 4}

\section{NGC 4395, the feeblest known Seyfert 1 nucleus ${ }^{\dagger}$}

\subsection{Introduction}

The dwarf Seyfert nucleus in NGC 4395 was first reported by Filippenko \& Sargent (1989) a decade ago. Optical spectroscopy showed high ionization narrow lines as well as broad permitted emission-lines. The detection of a compact radio source (Sramek 1992) added support to the idea that NGC 4395 is a feeble version of the more luminous Seyfert galaxies. Its low luminosity nucleus has a blue absolute magnitude $M_{B} \sim-11$, a luminosity $10^{4}$ times fainter than a classical Seyfert galaxy like NGC 4151. The detection of a featureless UV continuum gave further support to this hypothesis (Filippenko, Ho \& Sargent 1993). However, Shields \& Filippenko (1992), after several years of spectroscopic monitoring, reported that no evidence was found for continuum or line variability. Since variability is one of the most common characteristics of AGN, this result was quite surprising.

NGC 4395 is a nearly face-on dwarf galaxy $\left(B \sim 10.7, M_{B} \sim-17.9\right.$, assuming a distance of $5.21 \mathrm{Mpc}$ - see below) with morphological classification Sd III-IV in the extended Hubble system, as defined by Sandage \& Tammann in the RSA catalogue (Sandage \& Tammann 1981). It exhibits a star-like nucleus and an extremely low surface-brightness disk. The loose and disconnected spiral arms show some blue knots of star formation activity (for a colour plate see Wray 1988).

$\doteqdot$ This chapter is substantially the same as Lira et al. (1999). Any work by people other than the author of this thesis is noted. The text itself is also the work of the thesis author, with the usual contributions expected from the co-authors of the paper. 
As part of the multiwavelength imaging and spectroscopy study of the volume-limited sample of nearby galaxies, ROSAT HRI data and optical spectroscopy for NGC 4395 were acquired (see table 1.1). $B$ and $I$ broad-band images of the galaxy were obtained as part of an AGN monitoring program conducted by Dr. P. O'brien at Leicester University. Finally, HST and ROSAT PSPC data for NGC 4395 were retrieved from public archives. In this chapter the detection of optical and X-ray variability in the nucleus of NGC 4395 will be reported.

\subsection{Observations and Data Reduction}

A journal with the data used in this chapter can be found in table 4.1. It includes ground based and HST imaging and spectroscopy, and ROSAT data.

\subsubsection{HRI data}

As reported in chapter 2 an X-ray image of NGC 4395 was obtained with the High Resolution Imager (HRI) on board ROSAT on the 23rd of June 1996 as part of the AO7 cycle of pointed observations. The total exposure time was 11,253 secs.

Figure 2.24 shows a contour map of the central part of the X-ray frame overlaid on the Digital Sky Survey data for NGC 4395. The most prominent source in the figure (X2) has no obvious optical identification although the DSS image shows some diffuse emission in the area. The possible source $\mathrm{X} 1$ has been tentatively identified as the X-ray nuclear emission of NGC 4395. The slight shift between the optical nucleus and the X-ray emission is consistent with the accuracy of the ROSAT pointing. Indeed, another X-ray source approximately $7^{\prime}$ to the west of X2 and with a clear optical identification shows the same shift.

The count rates for $\mathrm{X} 1$ and $\mathrm{X} 2$ were computed as described in chapter 2 . The net count for X1 was $7.6 \pm 4.6$ photons, i.e., it is not a significant detection. For X2 a net count of $169.2 \pm 14.3$ photons was found. The count rates are $6.6 \pm 4.0 \times 10^{-4}$ and $15.0 \pm 1.3 \times 10^{-3}$ photons $\mathrm{s}^{-1}$ for $\mathrm{X} 1$ and $\mathrm{X} 2$ respectively. 


\begin{tabular}{|rccc|}
\hline \hline Date & Telescope & Mode & Archive Data \\
\hline 7 April 1988 & Hale & Spectroscopy & No \\
2 July 1992 & ROSAT PSPC & Imaging & Yes \\
15 \& 19 July 1992 & HST FOS & Spectroscopy & Yes \\
17 July 1992 & ROSAT PSPC & Imaging & Yes \\
5 December 1995 & HST WFPC2 & Imaging & Yes \\
5 - 16 June 1996 & JKT & Imaging & No \\
23 June 1996 & ROSAT HRI & Imaging & No \\
13 July 1996 & WHT & Spectroscopy & No \\
15 January 1997 & WHT & Spectroscopy & No \\
\hline
\end{tabular}

Table 4.1: Journal of observations of NGC 4395. †: Data provided by Filippenko et al. See Filippenko \& Sargent (1989).

\subsubsection{PSPC data}

As mentioned in chapter 2, the PSPC was one of the two focal plane instruments onboard ROSAT. The PSPC had an on-axis angular resolution of $\sim 25^{\prime \prime}$ and its energy resolution was $\Delta E / E=0.43 \times(E / 0.93)^{-0.5}$ (FWHM), where $E$ is in units of keV.

Two sets of PSPC data were retrieved from the ROSAT archive. One of these data sets is presumably that referred to as a private communication from Snowden \& Belloni in Filippenko, Ho \& Sargent (1993). The first set was obtained on the 2nd of July 1992 with 7,755 seconds of exposure time, while the second set was obtained 15 days later with 8,764 seconds exposure. Comparing both data sets it is easy to identify a variable $\mathrm{X}$-ray source which is consistent with the position of the nuclear source for NGC 4395 marginally detected from the HRI image.

For total count extraction of a PSPC point source, an aperture of $2^{\prime}$ should be adequate. Reducing the aperture to $1^{\prime}$ loses $15-20$ per cent of the counts at the soft end of the spectrum ( $\mathrm{E} \lesssim 0.1 \mathrm{keV}$ ) because of the wider PSF at lower energies (Hasinger et al. 1992). However, apertures larger than 30" around the NGC 4395 nuclear source would include other knots of X-ray emission, as can be seen in the HRI image. To estimate the flux due to these extra-nuclear sources, the net counts in the HRI image using an annulus centered on the nuclear source with an inner radius of $10^{\prime \prime}$ and an outer radius of $1^{\prime}$ were measured. A total of $23.5 \pm 14.7$ counts were found, so some contamination within the $1^{\prime}$ aperture is expected, but should not be significant.

For a $1^{\prime}$ aperture centered at the position of the NGC 4395 nucleus, the net counts were $67.2 \pm 10.7$ photons and $139.7 \pm 14.1$ photons for the first and second PSPC data sets, respectively. For the background estimation a large circle of radius $600^{\prime \prime}$ far away from 


\begin{tabular}{|c|c|c|c|c|c|c|c|c|c|c|}
\hline \multirow{3}{*}{ Date } & \multicolumn{4}{|c|}{ Blue Arm } & \multicolumn{6}{|c|}{ Red Arm } \\
\hline & [OII] & [NeIII] & {$[\mathrm{OIII}]$} & [OIII] & {$[\mathrm{OI}]$} & {$[\mathrm{OI}]$} & {$[\mathrm{NII}]$} & [NII] & [SII] & [SII] \\
\hline & $\lambda 3727$ & $\lambda 3869$ & $\lambda 4959$ & $\lambda 5007$ & $\lambda 6300$ & $\lambda 6363$ & $\lambda 6548$ & $\lambda 6583$ & $\lambda 6716$ & $\lambda 6731$ \\
\hline April $1988^{\dagger}$ & - & - & 64.41 & - & 23.02 & 7.59 & - & - & 19.77 & 22.97 \\
\hline July 1996 & 36.83 & 19.19 & 66.84 & 207.60 & 19.60 & 6.52 & 4.29 & 12.86 & 13.00 & 16.57 \\
\hline January 1997 & 36.34 & 21.55 & 69.72 & 227.82 & 24.69 & 7.77 & 4.80 & 14.40 & 16.34 & 19.81 \\
\hline
\end{tabular}

Table 4.2: Narrow line fluxes. Fluxes in units of $10^{-15} \mathrm{ergs} \mathrm{s}^{-1} \mathrm{~cm}^{-2}$.

$\dagger$ : Line fluxes from the spectrum acquired by Filippenko and collaborators.

\begin{tabular}{|l|ccccccccc|}
\hline \hline Date & $\mathrm{H} \beta_{N}$ & FWHM & $\mathrm{H} \beta_{B}$ & $\mathrm{FWHM}$ & $\mathrm{H} \alpha_{N}$ & $\mathrm{FWHM}$ & $\mathrm{H} \alpha_{B}$ & $\mathrm{FWHM}_{1}$ & $\mathrm{FWHM}_{2}$ \\
\hline April $1988^{\dagger}$ & 28.58 & 4.0 & 17.43 & 21.3 & - & - & - & - & - \\
July 1996 & 21.80 & 4.0 & 14.29 & 22.4 & 55.20 & 3.5 & 82.90 & 10.0 & 36.4 \\
January 1997 & 24.20 & 4.0 & 18.49 & 20.4 & 59.85 & 3.6 & 120.86 & 9.7 & 33.9 \\
\hline \hline
\end{tabular}

Table 4.3: Broad and narrow deblended line fluxes. Fluxes in units of $10^{-15} \mathrm{ergs} \mathrm{s}^{-1}$ $\mathrm{cm}^{-2}$.

$\dagger$ : Line fluxes from the spectrum acquired by Filippenko and collaborators.

any contamination by other X-ray sources was used. The associated count rates are $8.7 \pm 1.4 \times 10^{-3}$ photons $s^{-1}$ on the 2 nd of July 1992 , and $15.9 \pm 1.6 \times 10^{-3}$ photons s $^{-1}$ 15 days later, giving a variability of about a factor of two.

\subsubsection{Optical spectroscopy}

As reported in chapter 3 long slit spectra of the nucleus of NGC 4395 were obtained on the 13th of July 1996 and the 15th of January 1997. Although the conditions during January 1997 were not photometric throughout the night, the data for NGC 4395 were acquired during a clear period at the end of the night (see section 3.3.1).

The interstellar Galactic absorption towards NGC 4395 is just $A_{V}=0.02$ magnitudes (Schlegel, Finkbeiner \& Davis 1998), and no correction to account for this extinction is applied to the data in this chapter. The signal to noise per pixel achieved during July 1996 was $\sim 16$ for the red arm and $\sim 8$ per for the blue arm. For January 1997 the signal to noise per pixel was $\sim 42$ and $\sim 35$ for the red and blue arm, respectively.

As the NGC 4395 nucleus is unresolved for a $1^{\prime \prime}$ slit (see below) and the standard stars were observed with a wider slit $\left(8^{\prime \prime}\right)$, an empirical aperture correction was applied to the spectra. The correction was calculated by comparing the standard star spectra used in the flux calibration with spectra of the same stars obtained by software which simulated a narrow slit. A slight variation with wavelength was found and the spectra were scaled by a factor of $\sim 1.4$ at the blue end $(\lambda \sim 3700 \AA)$ and by a factor of $\sim 1.3$ at the red end 
$(\lambda \sim 6800 \AA)$.

HST Planetary Camera observations of the NGC 4395 taken in the narrow band F502N filter show that the $[\mathrm{OIII}] \lambda 5007$ emission region has a diameter of $\lesssim 0.4^{\prime \prime}$ (Filippenko, Ho \& Sargent 1993). In contrast, the FWHM for the spatial profile of an unresolved star in the spectroscopic data is $z 1^{\prime \prime}$. Inspection of the profile of the emission-lines in the spectra shows that the Narrow Line Region (NLR) is, as expected, spatially unresolved and no aperture effects have to be taken into account when comparing narrow line fluxes from the two spectra.

Measurements of the narrow line fluxes in July 1996 and January 1997 agree to within 25 per cent at worst (see table 4.2). Since a $1^{\prime \prime}$ slit has been used, the accuracy of the absolute calibration is expected to be of the order of $30 \%$. Comparison of the narrow line fluxes measured from the 1996-1997 data with the values measured from data obtained in 1988, and kindly provided by Filippenko and collaborators, have also been included in table 4.2 and 4.3 . The 1988 spectra were examined using the same IRAF packages used in the reduction the data reported in chapter 3 . The line fluxes agree to within $30 \%$, except for $[\mathrm{SII}] \lambda 6716$, where the difference is slightly bigger.

The blue spectrum obtained during the July 1996 observation shows a depression just after the [OIII] $\lambda 5007$ emission-line. This is due to the cosmetic defect in the LORAL CCD discussed in chapter 3 .

\subsubsection{Ground-based broad-band observations}

As part of an AGN monitoring program, NGC 4395 was observed on 5th-11th of June 1996 with the Jacobus Kapteyn Telescope (JKT). These observations were obtained, reduced and analyzed by Dr. P. O’brien.

JKT observations of NGC 4395 were obtained over a period of a few hours at the start of each night using the JKT CCD camera, using $B$ and $I$ band filters. An integration time of 5 minutes was used for each exposure. The atmospheric conditions were judged to be good and very stable over the entire week. This was confirmed by inspection of the extinction data recorded independently by the Carlsberg Meridian Circle, which showed the average $\mathrm{V}$-band extinction at the zenith was constant to \pm 0.01 over the observing run.

The CCD data were reduced in a standard way using the IRAF packages. Although photometric standard stars were observed during the monitoring campaign, the variability 
analysis was restricted to photometry of the nucleus of NGC 4395 relative to several stars within the same CCD frames. This procedure is better suited to searching for rapid, small-amplitude variability for which atmospheric changes can significantly affect the results (e.g., Done et al. 1990). Within each CCD frame several nearby stars of similar magnitude to the AGN nucleus were identified, and their counts calculated using a circular photometric aperture $6^{\prime \prime}$ in radius. A large aperture was used to ensure all the point-source light was enclosed allowing for possible small variations in seeing. For each star the sky background was removed by subtracting a scaled average of the counts in an annulus of inner and outer radius $9^{\prime \prime}$ and $11^{\prime \prime}$ respectively centered on the photometric aperture. A similar procedure was used for the AGN, except the annulus lay on top of the galaxy thereby permitting a first-order correction for the galaxy-light within the photometric aperture. No other attempt was made to correct for galaxy contamination. It is noted, however, that the central region of the galaxy of NGC4395 is quite faint even relative to the low-luminosity nucleus: the surface brightness of the galaxy in the nucleus vicinity is $\sim 20.5 \mathrm{mag} \operatorname{arcsec}^{-2}$. The light distribution is also flat in the spatial direction (i.e., no strong central bulge appears to be present). Hence the adopted galaxysubtraction procedure appears quite adequate for obtaining a good measurement of the intrinsic, nuclear-variability amplitude.

\subsubsection{HST WFPC2 observations}

NGC 4395 was observed with the WFPC2 onboard HST in the F450W ( B) and F814W $(\sim I)$ band filters on the 5 th of December 1995 as part of the GTO proposal 6232. These data were retrieved from the HST data archive and analysed using IRAF by Dr. R. A. Johnson. Results from a previous analysis of these observations can be found in Matthews et al. (1996). The nucleus of NGC 4395 was imaged on the PC chip which has a pixel size of $0.046^{\prime \prime}$. Three exposures of NGC 4395 were taken in each filter. The F450W exposure times were $1 \times 60 \mathrm{~s}$ and $2 \times 400 \mathrm{~s}$ and the $\mathrm{F} 814 \mathrm{~W}$ exposure times were $1 \times 60 \mathrm{~s}$ and $2 \times 300 \mathrm{~s}$. Only the short exposure observations have been used here as the nucleus was saturated in the long exposures. 


\begin{tabular}{|l|ccc|}
\hline \hline & HRI & \multicolumn{2}{c|}{ PSPC } \\
& & Low State & High State \\
\hline$\alpha=1.5$ & 0.37 & 1.11 & 2.06 \\
$\alpha=1.0$ & 0.32 & 1.10 & 2.05 \\
\hline \hline
\end{tabular}

Table 4.4: ROSAT $(0.1-2.4 \mathrm{keV})$ fluxes. Fluxes in units of $10^{-13} \mathrm{ergs} \mathrm{s}^{-1} \mathrm{~cm}^{-2}$.

\subsection{Results}

\subsubsection{X-ray imaging and spectral analysis}

The conversion to fluxes of the HRI and PSPC count rates for the NGC 4395 nuclear source were done assuming a power law spectrum $\left(F_{\nu} \propto \nu^{-\alpha}\right)$ with energy index $\alpha=1$ and 1.5, and using the energy range $0.1-2.4 \mathrm{keV}$. Adopting a Galactic hydrogen column density of $1.31 \times 10^{20} \mathrm{~cm}^{-2}$ (Stark et al. 1992) the HRI count rate gives an upper limit $\sim 3.5 \times 10^{-14} \mathrm{ergs} \mathrm{s}^{-1} \mathrm{~cm}^{-2}$, as can be seen in table 4.4. For a distance of $5.21 \mathrm{Mpc}$ this implies a maximum X-ray nuclear luminosity for NGC 4395 of $2.8 \times 10^{38} \mathrm{ergs} \mathrm{s}^{-1}$.

Since at least 100 counts are required to perform spectral analysis of PSPC observations, it was not possible to fit the data using the 34 energy channels of the detector. Instead, the total counts $(0.1-2.4 \mathrm{keV})$ for each data set were binned into a single channel to estimate the fluxes. A power law spectrum with $\alpha=1$ and 1.5 , and a hydrogen column density of $1.31 \times 10^{20} \mathrm{~cm}^{-2}$ were assumed. The results are shown in table 4.4.

In order to obtain spectral information from the PSPC data, the hardness ratio (HR) technique was used, which gives an 'X-ray colour' for objects with few net counts (Hasinger et al. 1992; Ciliegi et al. 1997). By definition $\mathrm{HR}=(\mathrm{H}-\mathrm{S}) /(\mathrm{H}+\mathrm{S})$, where $\mathrm{S}$ is the number of net counts within the channels $11-42(\sim 0.11-0.43 \mathrm{keV})$, and $\mathrm{H}$ is the number of counts in the channels $51-201(\sim 0.51-2.02 \mathrm{keV})$. Values of HR close to -1 indicate that the source has an extremely soft spectrum, while values close to +1 show that the source has a hard or heavily absorbed spectrum. For NGC $4395 \mathrm{HR}=0.34 \pm 0.11$, where the error was calculated as in Ciliegi et al. (1997). This value of HR, with a Galactic hydrogen column density, implies a spectral index of $\alpha \sim 1$. If NGC 4395 is indeed a typical Seyfert 1 an index $\alpha_{x} z 1.5$ is expected (Laor et al. 1997; Walter \& Fink 1993), which would require an additional hydrogen column density of $\sim 3 \times 10^{20} \mathrm{~cm}^{-2}$ to explain the observed HR. The hardness ratio for NGC 4395, therefore, is consistent with a modest intrinsic absorption of the soft part of the X-ray spectrum.

The PSPC data show a change by a factor of two in the X-ray flux over about 15 days. 


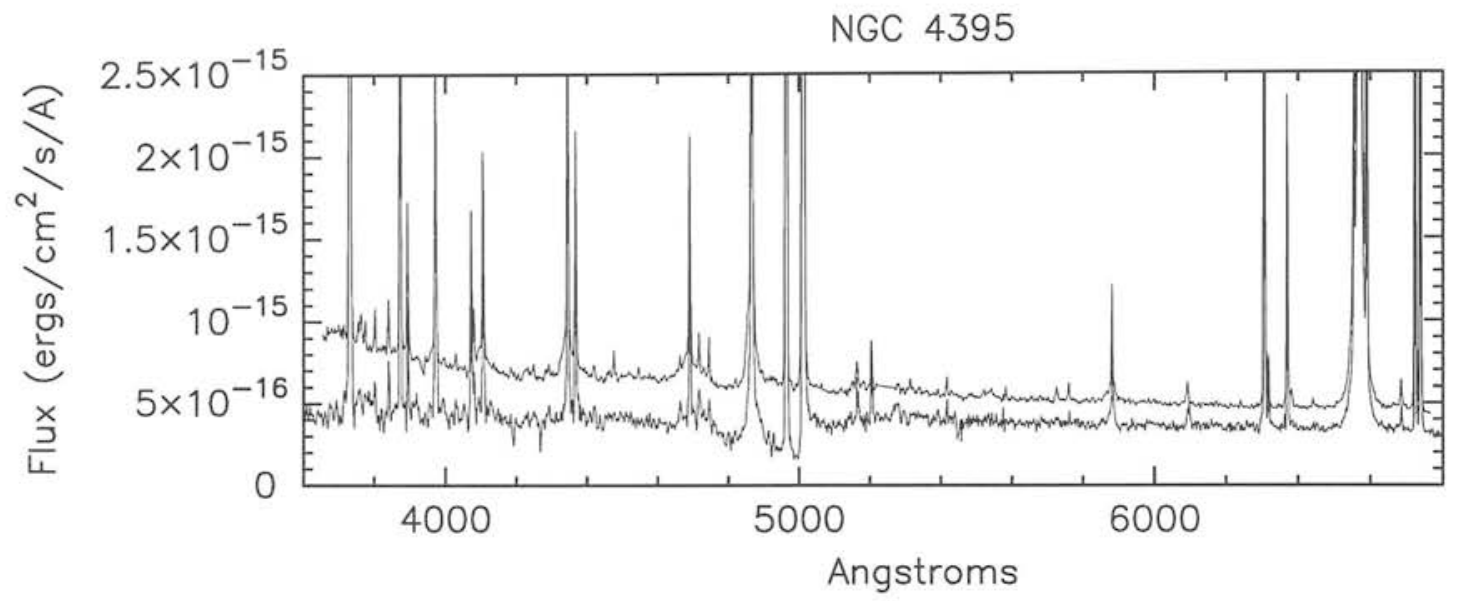

Figure 4.1: Optical spectra obtained with the WHT showing high and low state of NGC 4395. The lower spectrum was obtained on July 1996 and the upper was obtained almost exactly 6 months later. For plotting purposes, the blue continuum from the July 1996 spectrum has been slightly smoothed in order to suppress noise. Fluxes are in units of ergs s${ }^{-1} \mathrm{~cm}^{-2} \AA^{-1}$.

However, due to the the low number of counts the associated errors are large and the two measurements are consistent at a $3 \sigma$ level. The presence of variability could be confirmed by comparing the high PSPC and the HRI observations, since their $3 \sigma$ error bars do not overlap. This comparison cannot be done directly, however, due to the different responses of the detectors, with the PSPC being more sensitive at lower energies than the HRI. A soft enough spectrum could, in principle, explain both the observed PSPC and HRI count rates. This was assessed using PIMMS, an X-ray flux/count rate convertor distributed by the High Energy Astrophysics Science Archive Research Centre, HEASARC, at NASA. It was found that very soft incident spectra were required to explain the lowest count rate $(\mathrm{CR}-3 \sigma)$ of the high PSPC observation and the highest count rate $(\mathrm{CR}+3 \sigma)$ of the HRI*. Only for a power law with index $\alpha>2.0$ or thermal Bremsstrahlung emission with $k T>0.25 \mathrm{keV}$ can the two observations be reconciled. This result, however, is in disagreement with the observed HR. A power law index $\alpha_{x} \gtrsim 2.0$ implies a HR $<-0.2$, well below $(z 5 \sigma)$ the value found for NGC 4395 . 


\subsubsection{Analysis of optical spectra}

In this section evidence of variations in the continuum and emission lines of NGC 4395 will be presented.

For luminous Seyfert galaxies continuum variations are normally followed by flux changes in the broad emission lines. To achieve a reliable comparison of the line fluxes, an accurate (relative) calibration of the data must first be obtained. Also, a highly repeatable method has to be devised for the measurement of the fluxes. The calibration of the spectra is normally accomplished by using the flux in the narrow lines as an internal standard. Due to the large spatial extent of the NLR, any short-term variation in the ionising continuum is damped out and the line fluxes remain constant over the time scales of interest. A problem could arise, however, if the NLR is spatially resolved, covering a larger area on the sky than the size of the spectrograph entrance aperture, or if a very narrow slit is used. This means that the amount of detected NLR light would depend on the positioning of the slit and the seeing conditions during the observations (see discussion in Wanders et al. 1992). These issues will be discussed further in the remainder of this section in the context of the NGC 4395 observations.

Figure 4.1 shows the spectra obtained in July 1996 and January 1997. Within 6 months the continuum has changed by a factor of $\sim 1.3$ at red end of the spectra and by a factor of $\sim$ 2.2 at the blue end. The nuclear source becomes bluer when brighter, with a change in the spectral index from $\alpha \sim 2$ to $\alpha \sim 1$ (see section 4.4.1). From the narrow line fluxes quoted in table 4.2 it seems that the flux at the red end of the spectrum obtained in January 1997 might be slightly overestimated when compared to the July 1996 observation. It is then possible that there is negligible change at the red end of the spectra, and an even more dramatic change in colour between July 1996 and January 1997. The continuum becomes harder when it is brighter, which is a general characteristic of classical AGNs (Kassebaum et al. 1997; Kaspi et al. 1996; Reichert et al. 1994; Peterson et al. 1991; Edelson, Pike \& Krolik 1990).

The narrow line widths are not resolved in the data (resolution $\sim 3.5 \AA$ ). To find the fluxes given in table 4.2 the lines were fitted with a single Gaussian (except for the nitrogen doublet).

*If the observations are independent the likelihood of the true value being $3 \sigma$ or more from both is extremely small. Discrepancies of less than $2 \sigma$, implying an even softer spectrum, are needed for an overall probability corresponding to a " $3 \sigma$ difference". 

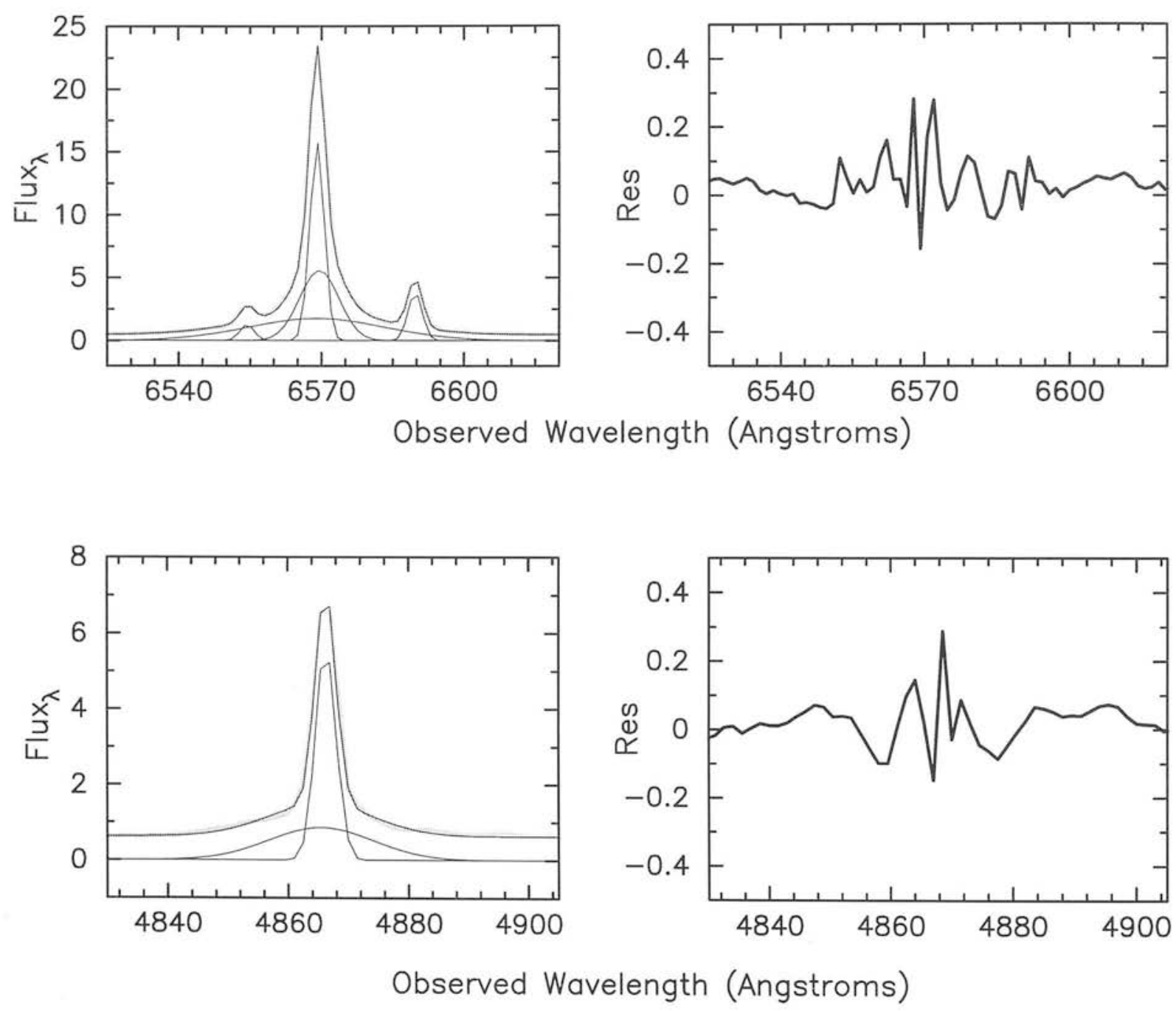

Figure 4.2: Gaussian profile fitting to $H \alpha$ (top) and $H \beta$ (bottom). Two and one broad components were fitted to each line, respectively. The narrow components had a fixed width measured from other narrow lines. The left panel shows the data (thick gray line), the fit (thin black line) and the individual model components (the continuum level is not included). The right panel shows the residuals between the data and model. All fluxes are in units of $10^{-15}$ ergs $\mathrm{cm}^{-2} \mathrm{~s}^{-1} \mathrm{~A}^{-1}$. 
Two and three Gaussians were then fitted to $\mathrm{H} \beta$ and $\mathrm{H} \alpha$ respectively: a narrow component with a fixed (instrumental) FWHM as measured from the narrow lines, and one (or two) free parameter broad components to fit the extended wings. The continuum level and slope were also free parameters during the fitting. A Lorentzian profile was also fitted to the broad components, but the results were much poorer than when using Gaussian profiles. Figure 4.2 shows the fit to $\mathrm{H} \alpha$ and $\mathrm{H} \beta$ as individual Gaussian components and as residuals between the data and model. Errors for the broad components were found to be less than 3 percent. They were computed as the square root of the diagonal elements of the covariance matrix of the non-linear model (i.e., they represent $68 \%$ confidence intervals for each parameter taken separately). Table 4.3 gives the fluxes and FWHM obtained for each line. For $\mathrm{H} \alpha$ the values for both fitted broad components are shown.

The error in the absolute line fluxes is probably of the order of 30 per cent, but as mentioned before, the relative changes in $\mathrm{H} \alpha$ and $\mathrm{H} \beta$ can be obtained to within a few percent by normalizing to nearby narrow line fluxes. At a distance of $5.21 \mathrm{Mpc}$ the NLR has a linear size of $\sim 10 \mathrm{pc}$ (from HST PC narrow band observations centred on the [OIII] $\lambda 5007$ line - Filippenko, Ho \& Sargent 1993). Although fairly modest, this diameter implies a traveling time of more than 30 years which should ensure that any variations in the central continuum source will be smeared out within the NLR and that the narrow line emission is fairly constant.

Given the observed size of the nuclear region (see sections 4.2 .3 and above) and the similar seeing conditions observed during both runs, there should be no significant aperture effects to take into account, so that the ratios of the narrow line fluxes observed in July 1996 and January 1997 can be used to assess any relative calibration differences. The observed narrow line ratios indicate that there is a slight variation with wavelength in the relative calibration. By fitting to these ratios, and assessing their scatter about the result, it is possible to estimate the significance of the broad line variations.

Figure 4.3 shows the narrow line ratios taken from table 4.2 and plotted against wavelength. The straight line is an unweighted best fit. If the two narrow line spectra are identical, apart from a linear flux correction, then the difference between the individual line ratios and this line reflect the noise in the data. The standard deviation of the scatter about the fit is 0.05 , shown as dashed lines.

The line ratios for the broad components of $\mathrm{H} \alpha$ and $\mathrm{H} \beta$ are also shown (filled stars) and clearly differ from the observed trend in the narrow lines. If the error in the ratio of the broad lines is similar to that inferred from the narrow lines then the significance of 


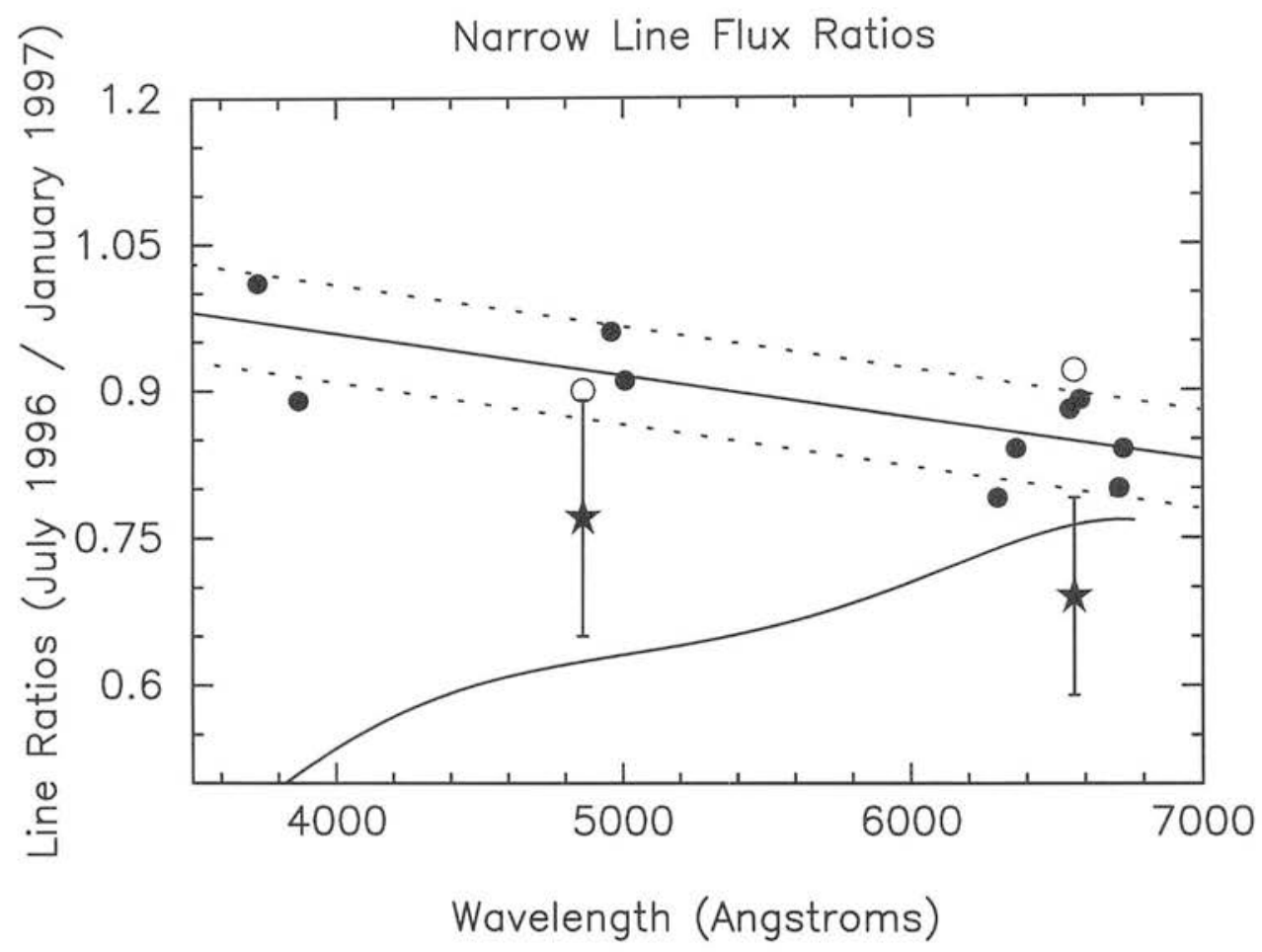

Figure 4.3: Narrow line flux ratios between July 1996 and January 1997 as a function of wavelength. A linear fit to the data is shown as a solid line. Dashed lines correspond to 1 standard deviation from the fit. The empty circles correspond to the ratio of the narrow line component of $\mathrm{H} \alpha$ and $\mathrm{H} \beta$ which were not used in the fit. The stars correspond to the ratio of the $\mathrm{H} \alpha$ and $\mathrm{H} \beta$ broad components with $2 \sigma$ error bars (see text). The ratio between the nuclear continuum observed in July 1996 and in January 1997 is also shown as a curved line at the bottom of the figure. 
the difference is estimated to be greater than 3 times the standard deviation. The ratio of the $\mathrm{H} \alpha$ and $\mathrm{H} \beta$ narrow components, which are consistent with the ratios found from the other narrow lines, have also been included in figure 4.3 (open circles). Applying the linear correction found using the narrow line flux ratios to those for the broad components, $\mathrm{H} \alpha_{B}^{96} / \mathrm{H} \alpha_{B}^{97}=0.81 \pm 0.05$ and $\mathrm{H} \beta_{B}^{96} / \mathrm{H} \beta_{B}^{97}=0.84 \pm 0.05$ are found, where the error has been assumed to be equal to the typical scatter of the narrow line ratios about the fit.

However, it is expected that the errors associated to the broad lines are somewhat larger than the ones observed in the narrow lines since a deblending process is required in order to measure the flux in the broad components. A crucial step in obtaining these fluxes is the adoption of the continuum level. Since this was a free parameter during the fit described above, two new fits were obtained for $\mathrm{H} \alpha$ and $\mathrm{H} \beta$, this time fixing the value of the adopted continuum level to $\pm 5 \%$ the best fit value. The new continuum levels can be seen in figure 4.4, where it is evident that they are a good representation of the noise in the neighbour regions of the lines. These adopted values are larger than 3 times the standard error associated to the best fit continuum level. The different broad component fluxes obtained with these continua correspond to changes in best fit values of $\pm 5 \%$ for $\mathrm{H} \beta$ and $\pm 2 \%$ for $\mathrm{H} \alpha$. Assuming that they are an estimate of the systematic error associated with the measurement of the line fluxes and summing in quadrature with the scatter observed in the narrow lines, we find that there is still compelling evidence that broad line variability has been detected, particularly for $\mathrm{H} \alpha$ (see figure 4.3).

Figure 4.3 also shows the ratio of the fitted continuum observed in July 1996 and January 1997 (see section 4.4.1) versus wavelength. This shows that there was a large variation in the blue, but that the variation in the red is only marginally significant.

Inspecting the spectra by eye, it appears that the broad components of higher order Balmer lines, such as $\mathrm{H} \gamma$ and $\mathrm{H} \delta$, have varied by a larger factor. However, it has not been attempted to quantify this given the noisiness of the data and the problem of blended lines.

An absorption line, identified as CaIIK $\lambda 3933$, can be seen near the blue end of the January 1997 spectrum (CaIIH $\lambda 3970$ coincides closely with $\mathrm{H} \varepsilon$, so is not easily seen). An additional tentative absorption line at $\sim 4055 \AA$ can be seen, for which there is no identification. These lines, as well as some other weak features, are not seen in the July 1996 spectrum due to the poor $\mathrm{S} / \mathrm{N}$ of the data (note that the spectrum in figure 4.1 has been slightly smoothed). The equivalent width of the observed CaIIK $\lambda 3933$ line is just above $1 \AA$. The line is quite broad, with a FWHM of $\sim 10 \AA$ ruling out the possibility of it 

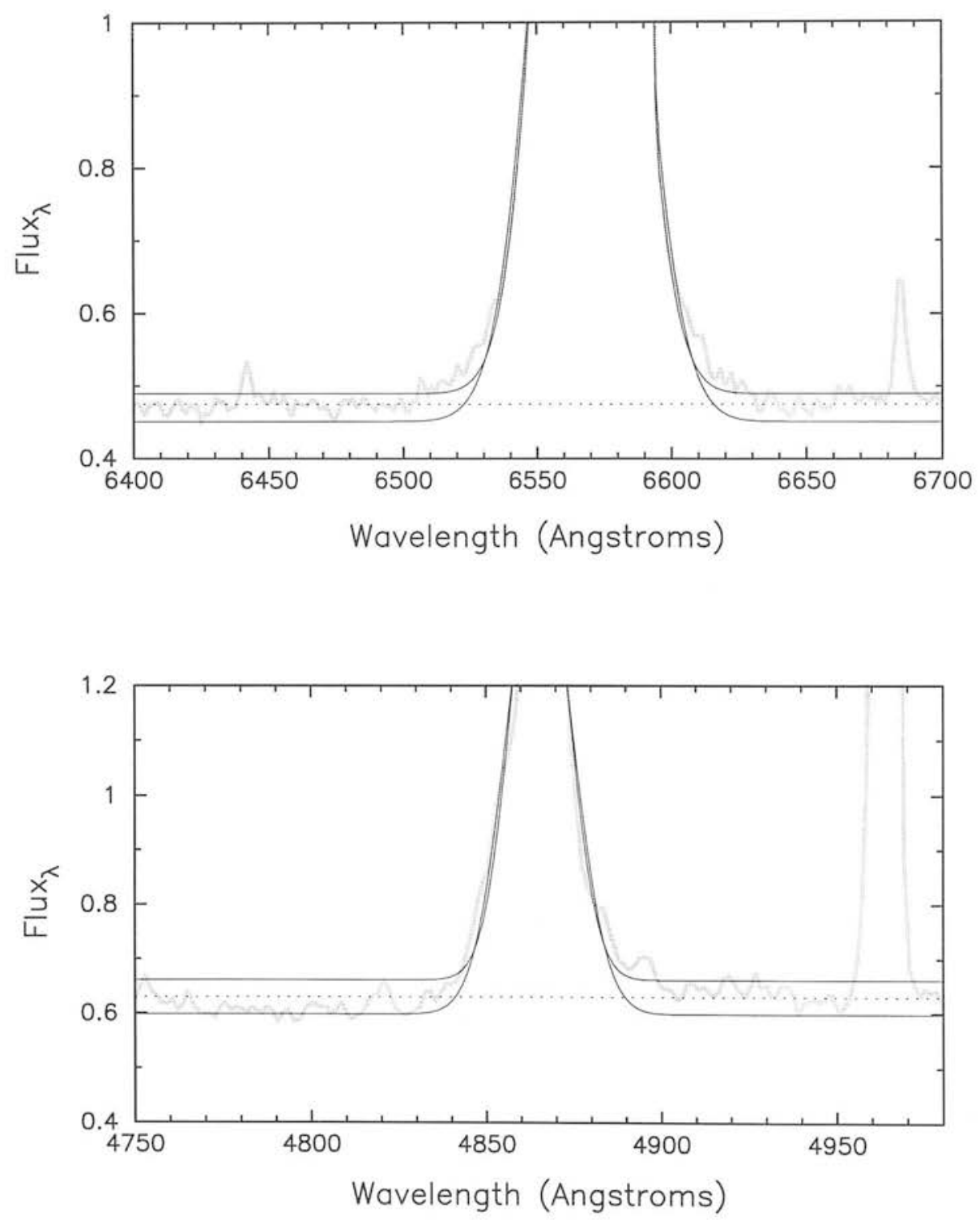

Figure 4.4: Fitted models to the $\mathrm{H} \alpha$ (top) and $\mathrm{H} \beta$ (bottom) emission lines. The adopted continuum levels correspond to $\pm 5 \%$ the best fit value (dashed lines). All fluxes are in

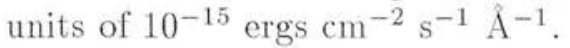


being caused by interstellar absorption. Its profile is slightly asymmetric, which may indicate more than one component. However, the quality of the data prevents any firm conclusions. There is no evidence of other important metal lines such as CN $\lambda 4200$, the G band of CH $\lambda 4301, \mathrm{MgI}+\mathrm{MgH} \lambda 5175$ or NaID $\lambda 5892$ in the data. Neither is the 4000 $\AA$ break observed, suggesting a very young stellar population. Bica (1988) shows that in a sequence of stellar population types from spiral galaxies the equivalent width (EW) of the CaIIK $\lambda 3933$ feature decreases towards younger populations as well as towards lower metallicities. The absence of the $4000 \AA$ break means that of Bica's templates only groups S6 and S7 can apply. For S7 most of the light is thought to come from populations of age $\sim 10^{8}$ years, and still has a EW of CaIIK $\lambda 3933 \sim 3.5 \AA$. Although it is not possible separate the effects of age and dilution in the case of NGC 4395, it seems likely that the population is young (conservatively $<1 \mathrm{Gyr}$ ) and has a dilution of at least $70 \%$.

The detection of the CaIIK absorption line suggests that other stellar features characteristic of young clusters may also be detectable. Among these, the near infrared CaII $\lambda \lambda 8498,8542,8662$ triplet should be the strongest (Terlevich, Diaz \& Terlevich 1990). A detection of the IR CaII triplet would provide confirmation of the presence of a young cluster. Direct measurement of the velocity dispersion would give its dynamical mass.

\subsubsection{Broad-band variability}

After careful examination of the JKT $B$ and $I$ images, no evidence was found for nuclear variability within any of the two hour observing windows each night. Therefore, the data for each night were averaged. To minimise any colour-dependent effects due to differences in the intrinsic spectral energy distribution of the nucleus, galaxy and stars, only data from frames for which the airmass was less than 1.5 were used to construct the nightly averages. The average airmass is very similar for each night as the observing periods were at similar UT.

The average $B$ and $I$ band CCD count ratios for two stars, and for the nucleus and one of the stars are shown in figure 4.5. The values have been normalised to unity using the data from the last four nights of the run. Based on the ratios between the two stars (empty circles), the one-sigma uncertainty for flux variability is estimated to be $2 \%$. Clearly, the nucleus is variable over the first few nights, with the largest change being a brightening by about 20 percent from nights two to three. The changes are similar in form and amplitude in both bands. Other small-amplitude variations are possibly present during the second half of the week, although these are not highly significant. 


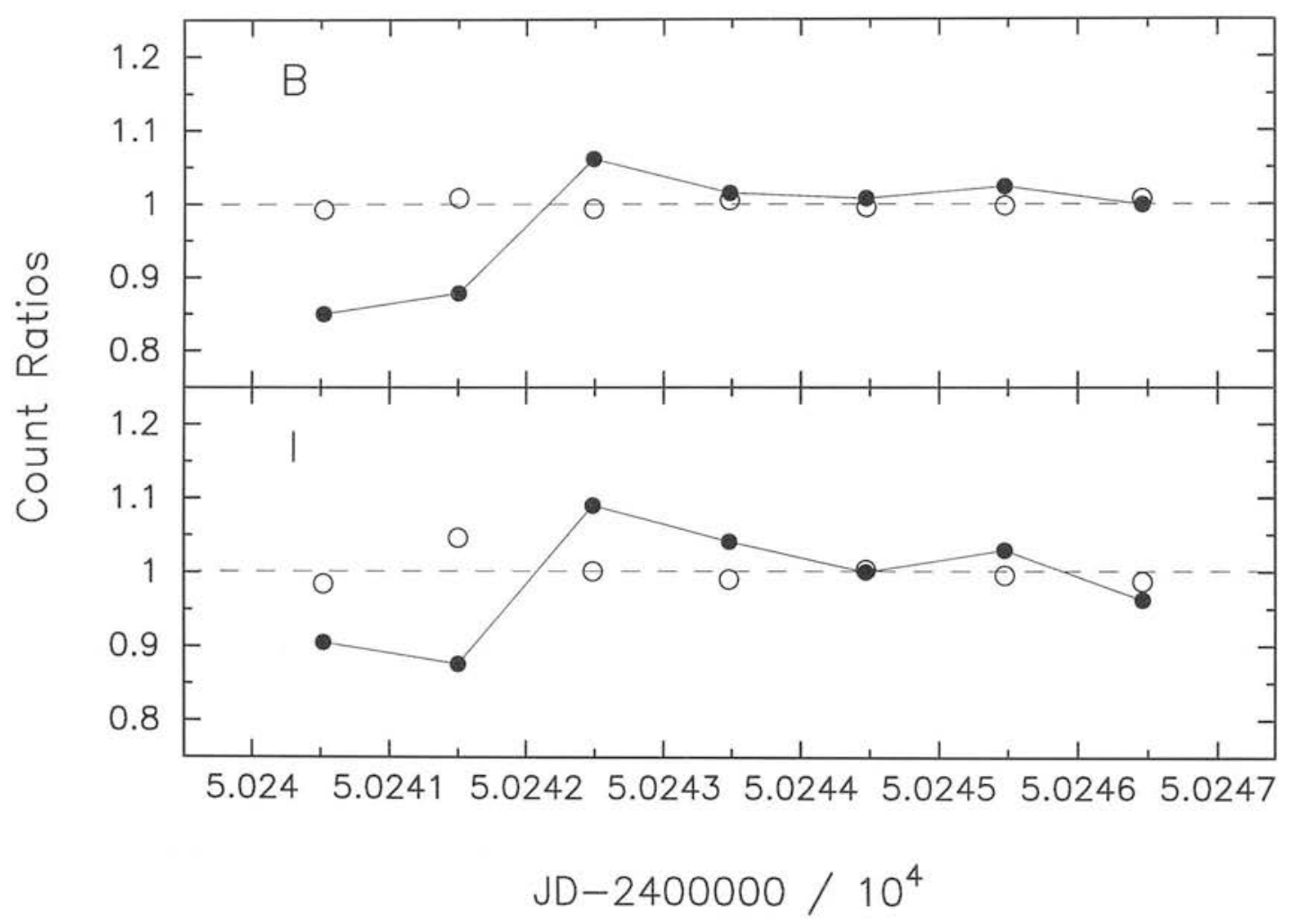

Figure 4.5: JKT $I$ and $B$ relative photometry for the NGC 4395 nucleus. The count ratios between two field stars (open circles) are compared with the ratio between the nuclear photometry and one of the stars (filled circles). The star-star comparison suggests a typical error of $\sim 2 \%$ so the variation in the nuclear photometry is significant. 
Absolute photometric magnitudes were derived using several flux standards and field stars, all observed at very low airmass $(<1.1)$, so colour differences are insignificant. This implies nuclear (galaxy subtracted) magnitudes of $B=16.8$ and $I=15.8$ for NGC 4395 during the JKT run. One-sigma errors of \pm 0.1 magnitudes are estimated. Filippenko \& Sargent (1989) quote a B magnitude of $~ 17.3(0.42 \mathrm{mJy})$ based on the flux density at $4400 \AA$ from their spectroscopic data. From the spectroscopy shown in figure 4.1, the fluxes at $4400 \AA$ imply B magnitudes of 18.0 and 17.5 for July 1996 and January 1997 respectively. This implies a historical flux variation by a factor $\sim 2$, and strongly supports the detection of variability in NGC 4395 .

\subsubsection{Analysis of HST images}

Photometry in standard bands was derived from the HST observations using a circular aperture of $1^{\prime \prime}$ diameter. The background emission was negligible. This gave values of $B=16.91$ and $I=16.23$, comparable to the values from the JKT broad-band imaging, further supporting evidence of variability.

HST imaging has been previously discussed by Filippenko, Ho \& Sargent (1993). However those observations were taken by WFPC1, whereas the data discussed here were taken with WFPC2. Therefore, the images were examined for any sign of resolved structure. Model point spread functions (PSFs) for both the F450W and F814W filters at the relevant chip positions were calculated using Tiny Tim software. Standard IRAF routines were then used to find the centroids and radial profiles of both the real and model data. Comparison of HST data with model PSFs in the very central regions is very sensitive to centroid location with respect to pixel centres, as even the PC is somewhat undersampled. Proper modelling was not attempted here. Instead the model PSF was scaled by eye to match the real data at radii of 1-2 pixels, in order to look for evidence of extended structure.

The F814W data have a clear peak pixel, so a direct comparison with a PSF is relatively secure. The comparison with the PSF is shown in figure 4.6. It can be seen that the core of the PSF matches the data very well, so the finding of Filippenko, Ho \& Sargent (1993) that most of the flux from the nucleus of NGC 4395 comes from an unresolved point source is confirmed. A proper fit has not been found, but an intrinsic FWHM of more than about half a pixel would have been easily detected. At the adopted distance of $5.21 \mathrm{Mpc}$ this corresponds to a physical size of $0.6 \mathrm{pc}$.

In the wings of the profile, however, the source has a clear excess above the PSF. Although this is a very small number of counts, it is a large factor above the predicted wings, so 
HST WFPC2 814W filter : radial profile of NGC 4395
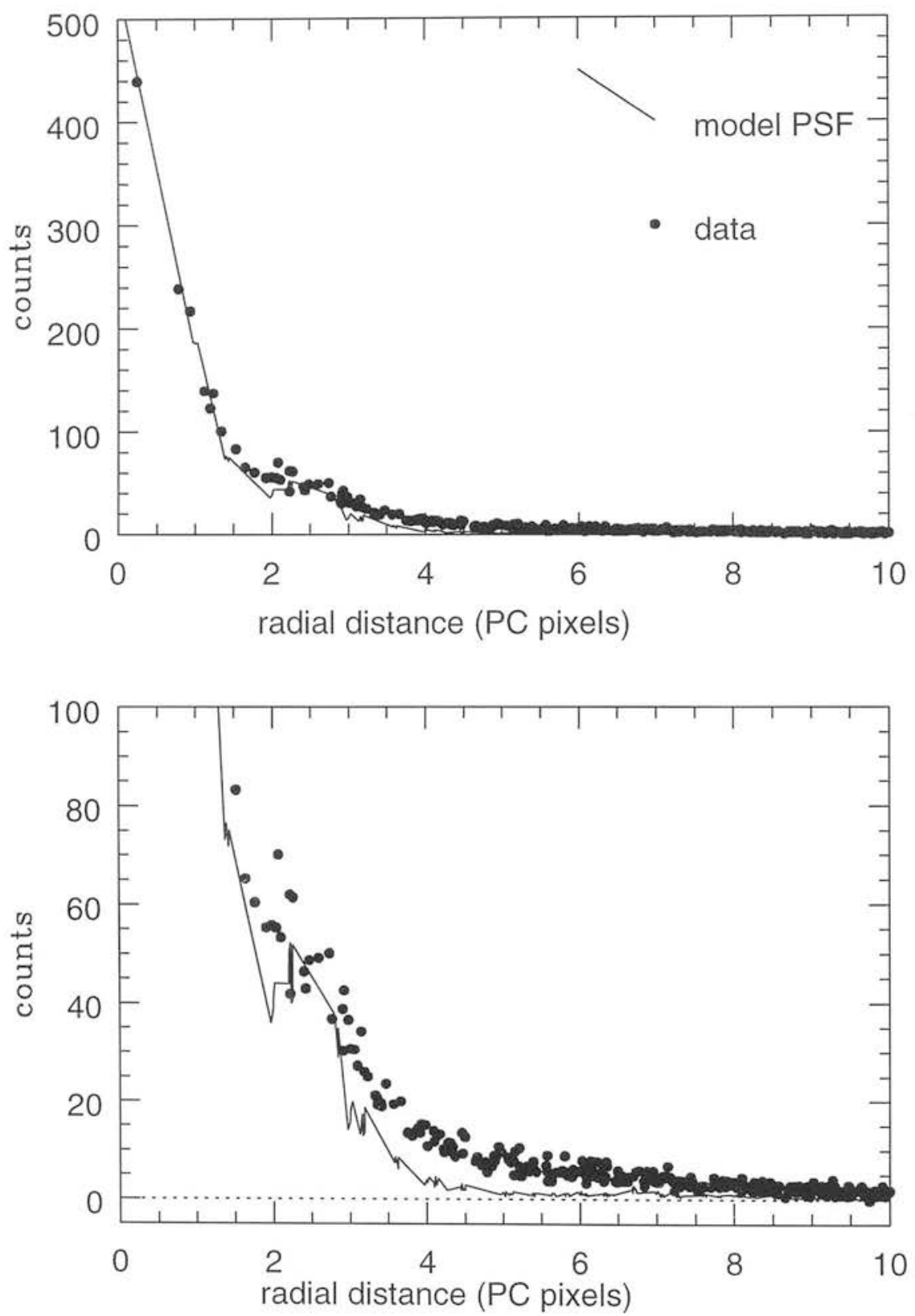

Figure 4.6: Comparison of the radial profile of the NGC 4395 nuclear emission and model Tiny Tim psfs. The nucleus of the galaxy was imaged onto the HST PC 2 using the F814W filter. The bottom panel shows a detail of the top panel, so that the extended component can be seen more clearly. 
it is unlikely to be accounted for by a slightly different normalisation of the PSF without disagreeing badly with the core. In this region the Tiny Tim PSF should be very reliable and the spacecraft jitter during these observations was only 0.14 pixels. There is, then, evidence of diffuse emission surrounding the core of NGC 4395. Without proper modelling it would be unwise to quantify the excess, but very roughly the integrated diffuse flux could be as much as 10 per cent of the core flux. Its diameter of $\sim 18$ pixels $=0.8^{\prime \prime}=$ $20 \mathrm{pc}$ is similar to the size of the extended [OII] emission detected by Filippenko, Ho \& Sargent (1993) - see also Matthews et al. (1996). Indeed, it is quite possible that at the $5-10 \%$ fraction of the core considered here, the diffuse light in the F $814 \mathrm{~W}$ filter is entirely due to nebular emission. On the other hand, the detection of an underlying young stellar component (see section 4.3.2) could explain the diffuse emission as contamination by a nuclear stellar cluster.

In the blue (F450W) observation, the data has two bright pixels and any point source is probably centred somewhere between these. The type of crude analysis used above is, therefore, even less reliable, and so a detailed discussion of this image is not given. Very roughly however, the data are at least consistent with the same story - an unresolved core and diffuse emission at the $10 \%$ level.

\subsection{Discussion}

\subsubsection{Spectral energy distribution}

For the determination of the Spectral Energy Distribution (SED) of NGC 4395, ultraviolet HST spectra obtained with the Faint Object Spectrograph (FOS) in July 1992 were retrieved from the public archive. The original data were published by Filippenko, Ho \& Sargent (1993).

To determine the featureless UV and optical continuum a low degree polynomial was fitted to the spectra, allowing for the rejection of all the emission-line features during the fitting procedure. The resulting UV continuum will include broad quasi-continuum such as Balmer continuum and FeII emission-lines. Some starlight contamination is expected as stellar signatures have been observed in the nuclear emission but it is only of the order of $10 \%$ of the total flux at $4000 \AA$ (see sections 4.3 .2 and 4.3 .4 ).

For the X-ray data an effective energy for the ROSAT HRI bandpass of $0.8 \mathrm{keV}$ was deduced assuming a power law spectrum of index $\alpha=1.5$. For the PSPC the value 


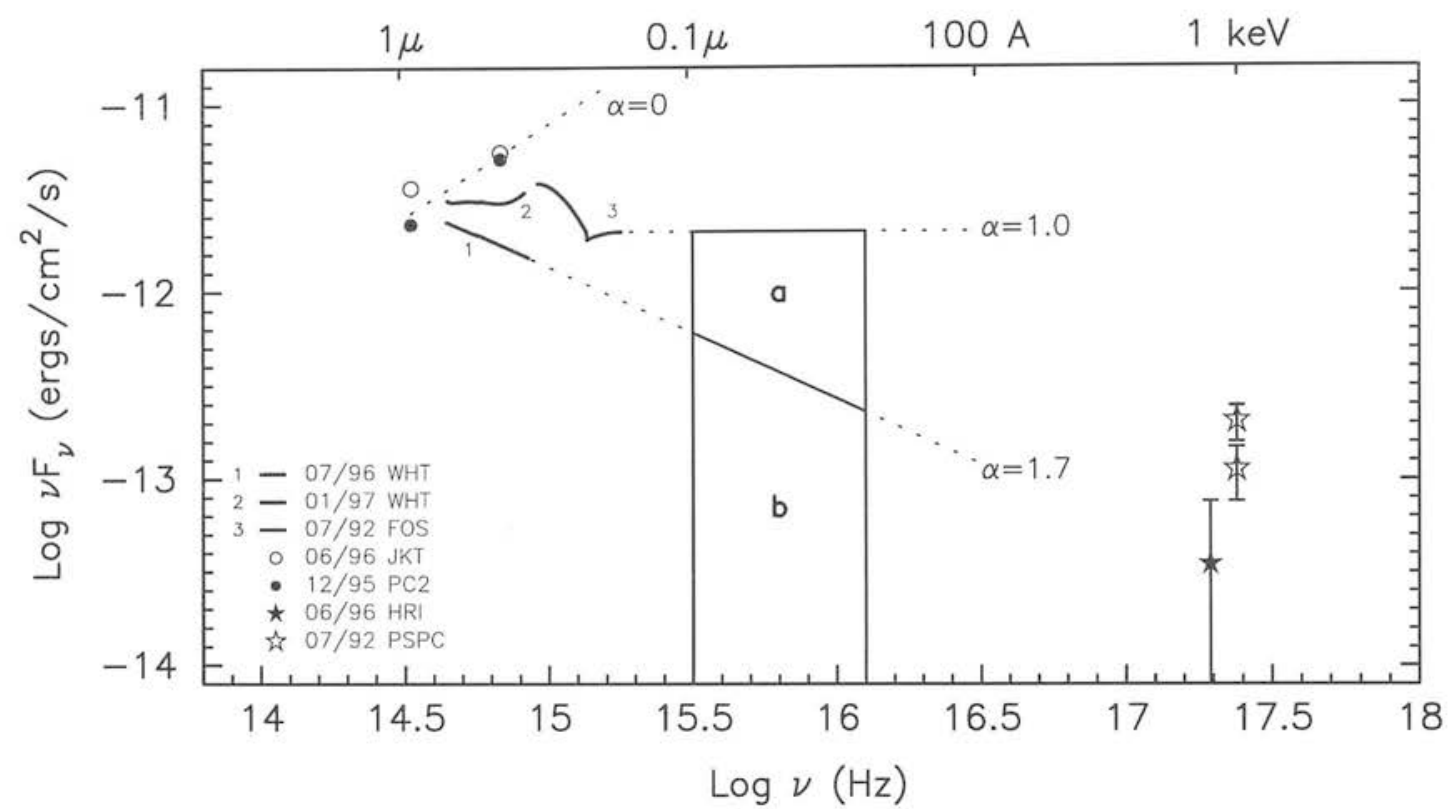

Figure 4.7: Spectral energy distribution of the nucleus of NGC 4395. Dotted lines have been drawn as examples of power law functions with energy spectral indices $\alpha=0,1$ and 1.7. The ionizing continuum (between $\nu=3.3 \times 10^{15}$ and $\nu=10^{16} \mathrm{~Hz}$ ) has been represented by the areas a and b (see text). The optical spectra (lines 1 and 2 ) were obtained in July 1996 and January 1997 and show a low and high state of activity. The FOS UV spectrum (line 3) was retrieved from the HST public archive (it has been published by Filippenko, Ho \& Sargent 1993). B and I broad band observations are shown as open circles (JKT data) and filled circles (HST data). The best estimate of the measured ROSAT HRI $\mathrm{X}$-ray flux is indicated with a filled star. Archive ROSAT PSPC fluxes are indicated with open stars. All the X-ray data are given with $2 \sigma$ error bars. The figure key shows the dates (month/year) of the different observations. Note from table 4.1 that the X-ray observations are not simultaneous with any other data.

adopted was $1.0 \mathrm{keV}$.

Figure 4.7 shows the observed SED for NGC 4395. Dashed lines have been drawn as examples of power law functions with spectral indices $\alpha=0,1$ and $1.7\left(f_{\nu} \propto \nu^{-\alpha}\right)$. Several striking features are evident in the SED plot. The change in the continuum shape between the two spectroscopic observations obtained in 1996 and 1997 is dramatic. The featureless optical continuum obtained in July 1996 is very steep, with a best fitted spectral index $\alpha \approx 1.7$. The extrapolation of this continuum to shorter wavelengths seems to agree with the observed X-ray luminosities. Compared with more luminous AGN, NGC 4395 seems to be a very quiet X-ray source, unless the PSPC and the HRI observations were made during extremely low activity states. 
The optical continuum looks flatter and brighter by January 1997 with a best fitted $\alpha \approx$ 1.1, but starts to raise for wavelengths shorter than $4000 \AA$. The HST UV data from 1992 shows a sharp break at $\sim 2200 \AA$. After the break the UV spectrum seems to agree with an $\alpha=1$ spectral distribution. The bump observed at $\sim 3000 \AA$ nicely matches the optical observations of 1997. However, due to the uncertainties in the spectral absolute flux calibration and the 4 year gap between the optical and the UV observations, the match could be simply fortuitous.

The 3000 A UV bump in figure 4.7 resembles the 'Small Blue Bump' observed in many other classic AGN (Wills, Netzer \& Wills 1985), which is believed to be a blend of broad permitted FeII lines and Balmer continuum (Bac). The UV excess corresponds to $4.0 \times 10^{-13}$ ergs $\mathrm{cm}^{-2} \mathrm{~s}^{-1}$ (obtained by integrating below the SED between 2220 to 4500 $\AA$ and subtracting the flux of the underlying continuum approximated as a power law), giving an estimation for the UV excess $/ \mathrm{H} \alpha$ ratio of $\sim 2.5$. Observed ratios for more powerful AGN range from $\sim 2$ to 5 , in agreement with the observations (Edelson \& Malkan 1986; Malkan 1983). These values are much larger than the model predictions for Case B recombination (Kwan \& Krolik 1981), suggesting that perhaps more than the 50 per cent of this UV excess comes from FeII emission. The lack of observed FeII features in the optical range in NGC 4395 is not umusual. Wills, Netzer \& Wills (1985) and Netzer et al. (1985) have shown that strong UV Fell emitters can show extremely weak optical FeII lines.

The $\sim 2200 \AA$ break can also be explained as substantial internal reddening in NGC 4395 . Using the extinction curve by Cardelli, Clayton \& Mathis (1989b) it is found that a visual absorption $A_{V} \lesssim 0.4$ is required to produce the dip at $2200 \AA$. The dust responsible for this extinction is probably not located in the Broad Line Region (BLR) since it is unlikely to survive the high densities and temperatures. Nor does the observed Balmer decrement support the presence of significant dust in the NLR: from table $4.2 \mathrm{H} \alpha_{N} / \mathrm{H} \beta_{N} \sim 2.5$ is found, while an extinction $A_{V} \sim 0.4$ implies $\mathrm{H} \alpha_{N} / \mathrm{H} \beta_{N} \sim 3.3$. Rowan-Robinson (1995) claims that the dust responsible for optical and UV reddening in quasars, with $A_{V} \sim$ $0.1-0.5$, is diffuse material located in the NLR or associated with the interstellar medium of the parent galaxy. The latter seems to agree with the observations reported here, although an $A_{V} \sim 0.4$ is slightly high for a nearly face on galaxy.

It seems that NGC 4395 has been caught in three different states. The broad band JKT data and the HST images show a bright and blue source with $\alpha \sim 0$, similar to luminous quasars. The January 1997 data show a state with $\alpha \sim 1$ and a much less obvious 'Big Blue Bump'. Finally, in July 1996, NGC 4395 shows a steep spectrum with $\alpha \sim 2$. 
The relative strength and slope of the Blue Bump can vary widely amongst quasars and Seyfert galaxies (Elvis et al. 1994; Walter \& Fink 1993; Puchnarewicz et al. 1996). In the NGC 4395 medium state, the observed continuum with $\alpha \sim 1$ is in fact quite similar to that seen in many Seyfert galaxies (e.g., Edelson \& Malkan 1986; Malkan 1983; Kriss et al. 1991). The low state spectrum of July 1996 is much more unnusual, but not unprecedented - for example, the ultraluminous IRAS galaxy F10214+4724 and the archetypal Type-2 Seyfert NGC 1068 both show steep spectra throughout the near-IR to UV (see Lawrence et al. 1994 and references therein). More recently two other extremely low luminosity broad-line AGN have been found to have a steep optical-UV spectrum M81 (Ho, Filippenko \& Sargent 1996), and NGC 4579 (Barth et al. 1996). In both these cases the X-ray luminosity is very strong, with $\alpha_{o x}=0.92$ and 0.86 respectively. However, for NGC4395, the continuum seems to continue falling steeply all the way to soft X-rays $\left(\alpha_{o x} \sim 1.9-\right.$ see section 4.4.6).

\subsubsection{Optical spectroscopic variability}

For the first time optical variability has been reported for NGC 4395. Previous observations had given negative results for any change in the continuum level or line fluxes (Shields \& Filippenko 1992), although this was not quantified. The data reported here show a substantial change in the optical continuum (3700 - $6700 \AA)$, as can be seen in figure 4.1. It seems that NGC 4395 moves between high and low activity states, characterized by spectral distributions with indices $\alpha \approx 1.0$ and $\alpha \approx 1.7$, respectively. The broad-band data suggest an even higher state with $\alpha \sim 0$.

As was mentioned earlier, a significant variation in the $\mathrm{H} \alpha$ and $\mathrm{H} \beta$ broad line fluxes has been detected, but with an amplitude much smaller than the variation exhibited by the continuum. Variability studies for local Seyfert galaxies show that the amplitude of the flux variations changes from line to line, with the high ionization lines showing the largest variations (Carone et al. 1996; Clavel et al. 1991; Kassebaum et al. 1997). While a variation of $\sim 1.5$ is normal for the total $\mathrm{H} \beta$ flux, a factor of up to 16 has been observed for HeII $\lambda 4686$. From table 4.3 the (uncorrected - see section 4.3.2) ratio of the 1996 fluxes to 1997 fluxes is 1.2 for $\mathrm{H} \beta$ and 1.3 for $\mathrm{H} \alpha$.

Rosenblatt et al. (1992) carried out optical spectroscopic monitoring of 13 Seyfert galaxies with $5 \times 10^{39}<\mathrm{L}(\mathrm{H} \beta)<4 \times 10^{42} \mathrm{ergs} \mathrm{s}^{-1}$ from 1979 to 1984 . They found strong evidence that variability in the continuum and $H \beta$ occur on time scales of 90 days or less. They also found that less luminous galaxies are more strongly variable in their lines 


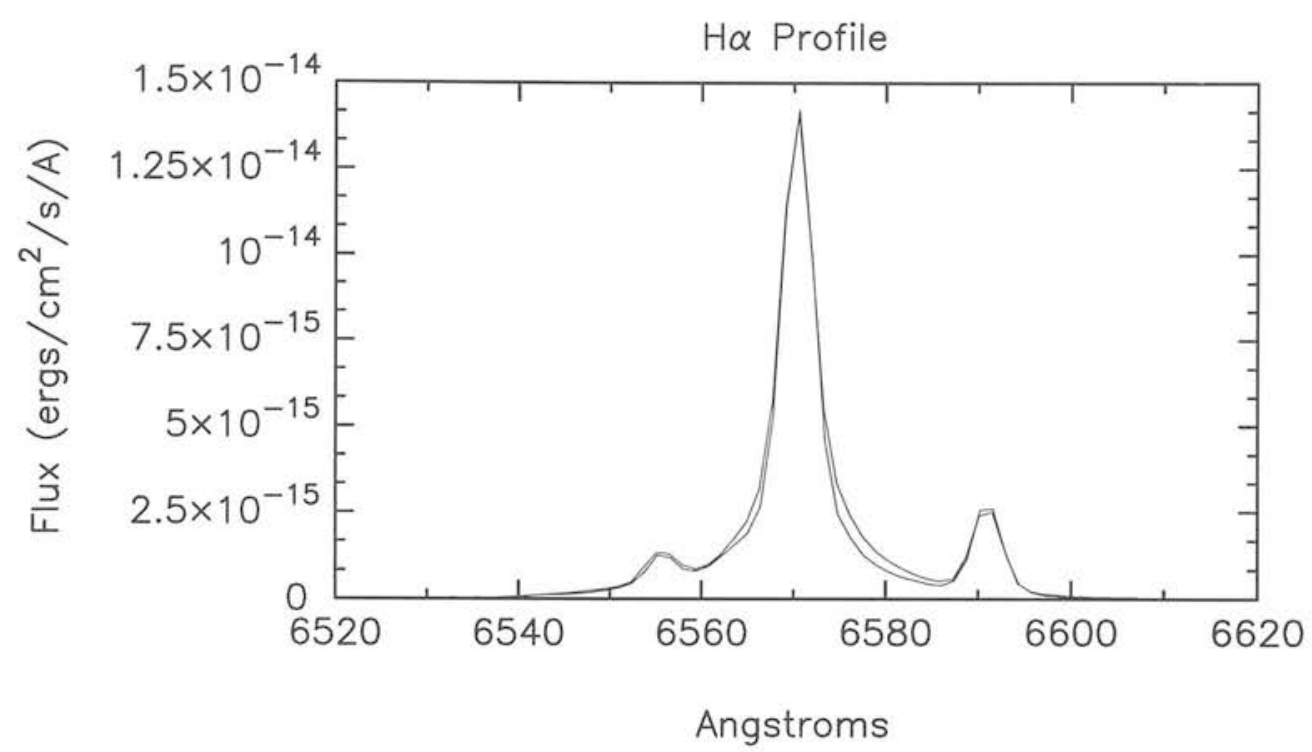

Figure 4.8: $\mathrm{H} \alpha+\mathrm{N}$ II line profiles for the high and low state of NGC 4395. The continuum have been subtracted from both spectra and a scale factor of 1.2 has been applied to the low state data.

and continuum. This report on optical variability for NGC 4395 shows that extremely low luminosity Seyfert galaxies do vary, but it is still unclear whether they follow the pattern shown by the more luminous objects. Rosenblatt et al. (1992) also show that in 8 galaxies peak-to-peak changes in $\mathrm{H} \beta$ fluxes were $100 \%-200 \%$, while the continuum varied by $200 \%-350 \%$ for 12 of the 13 galaxies, in agreement with the observations for NGC 4395 .

No change in the line profiles is observed between the two epochs, as can be seen in figure 4.8, where the $\mathrm{H} \alpha+\mathrm{N}$ II lines from July 1996 and January 1997 have been plotted together, after continuum subtraction and a scaling of the former by a factor of 1.2. It has been shown for NGC 5548 that while emission-line fluxes vary with changes in the ionizing continuum, the line profiles do not reflect these variations (Wanders \& Peterson 1996; Kassebaum et al. 1997). Instead, it is expected the timescale for line profile changes to correlate with the BLR kinematical evolution timescale.

As has been noted, it is a common property of Seyfert galaxy variability that the optical continuum becomes harder when the source becomes brighter. However, it has always been worrying that this might be due to stellar contamination in the red. For NGC 4395, the surrounding bulge is of very low surface brightness and Filippenko, Ho \& Sargent (1993) stress the featureless nature of the continuum, suggesting that any contribution from an old stellar population is small. The discovery of CaIIK $\lambda 3933$ absorption (section 4.3.2) and a diffuse component to the nuclear broad-band emission (section 4.3.4) suggests 
that a small but significant fraction of the light (10-20\%) comes from a very young stellar cluster, but this is unlikely to produce the colour dependence observed in variability. An important test will be high $\mathrm{S} / \mathrm{N}$ spectroscopy in the low state, when the CaIIK absorption should be larger.

\subsubsection{Fast variability}

In the X-rays, a factor two variation in 15 days have been seen. By the standards of local AGN, this is not particularly fast (for example, NGC 4051 can change by a factor of two in half an hour - see Papadakis \& Lawrence 1995). Likewise, optical spectroscopic variability by a factor of two in six months is quite reasonable for local Seyfert galaxies like NGC 5548. Luminous quasars variations are slower, taking typically several years to vary by a factor of two - see for example the light-curves in Hawkins (1996). However, evidence that NGC 4395 varies in a more dramatic fashion than classical Seyfert galaxies is presented here.

First, it has to be noted that the last night of the broad-band imaging (JKT) observations were taken only 32 days before the night in which the spectroscopic low state is found. During this time the B-band flux decreased by a factor of three. Variations by a factor of three in the UV over 50 days certainly occur in Seyfert galaxies - for example in the well known 1989 monitoring of NGC 5548 (Clavel et al. 1991) - but over the same period, the blue optical flux (at $4870 \AA$ ) followed the same pattern of variation with a much smaller amplitude, about a factor 1.5 (Peterson et al. 1991). The largest optical variations seen in the four year 1988 - 1992 optical monitoring of NGC (Peterson et al. 1994) are by a factor two over about 100 days.

The second piece of evidence that NGC 4395 varies more dramatically and/or more quickly than more luminous AGN is that a $20 \%$ change from one night to the next during the broad-band imaging observations has been seen. Very few short-timescale monitoring campaigns have been carried out. Optical monitoring of the low luminosity Seyfert NGC 4051 by Done et al. (1990) found the B-band flux to be constant within 1 per cent over a whole week. Likewise, the two week long monitoring of NGC 4151 by Edelson et al. (1996) showed that variations at $5125 \AA$ were 1 per cent or less. During the same period however, UV variations were considerably larger, with a normalised variability amplitude of 9 per cent at $1275 \AA$, and one particular event showing a rise of $\sim 20$ per cent over 1 day.

There is, then, tentative evidence that NGC 4395 either varies more quickly or with larger amplitude than more luminous AGN. However, especially for the intra-week monitoring, 
we may have seen a freak event. Repeated monitoring is clearly needed to see what is typical. UV and fast X-ray monitoring would also be of great interest.

\subsubsection{The ionizing continuum}

As noted in section 4.4.1, the blue bump in NGC 4395 is occasionally strong, and sometimes very weak. Is the implied ionising continuum luminous enough to produce the observed broad Balmer lines? This depends sensitively on how one extrapolates into the UV. If a power-law spectrum $L_{\nu}=L_{\nu_{0}}\left(\nu / \nu_{0}\right)^{-\alpha}$ is assumed, where $L_{\nu_{0}}$ is the monochromatic luminosity per unit frequency at the hydrogen ionization edge $\nu_{\circ}$, then extrapolating to infinity gives the number of ionising photons $N_{\text {ion }}=L_{\nu_{0}} / h \alpha$ (some authors extrapolate to the He edge at $228 \hat{\AA}$ but this makes relatively little difference). $L_{\nu_{0}}$ can in turn be estimated by extrapolating from the observed optical/UV continuum. Then on standard Case B assumptions, with a unity covering factor, and assuming that all the ionising photons are absorbed, $N_{i o n} / N_{H \alpha}=2.2$. More realistic conditions should change this predicted value by less than a factor two. Relaxing the other assumptions (such as complete coverage) makes $N_{i o n} / N_{H \alpha}$ larger.

For the medium state, it is possible use the $\mathrm{H} \alpha$ luminosity observed in January 1997, and calculate $N_{i o n}$ by extrapolating the HST UV luminosity at $2000 \AA$ with $\alpha=1$. (This corresponds to the sum of areas $a+b$ in figure 4.7 where, for the purposes of the plot, the He edge has been adopted as the upper limit for the ionizing continuum; the actual determination of $N_{i o n}$ was done by integrating to infinity). This gives $N_{i o n} / N_{H \alpha}=$ 1.5 which, within the uncertainties of this calculation, is just consistent with $\mathrm{H} \alpha$ being produced by photo-ionisation. For the low state, the $\mathrm{H} \alpha$ luminosity observed in July 1996 is used (which is only 0.8 times smaller than the $H \alpha$ luminosity in the high state). An optimistic extrapolation from the blue end of the optical spectrum with $\alpha=1$ gives a result very similar to the case above. However, if the observed steep spectrum continues falling as $\alpha=1.7$ (area a in figure 4.7 ) it is found that $N_{i o n} / N_{H \alpha}=0.4$, and the deduced ionising luminosity fails to explain the observed broad $\mathrm{H} \alpha$ by a substantial factor.

Such a deficit of ionising photons has been claimed for Seyfert 2 galaxies (e.g., Wilson, Ward \& Haniff 1988; Kinney et al. 1991) and for the extended emission-line regions of radio galaxies (e.g., Robinson et al. 1987), where it has been used to argue for an obscured and/or anisotropic continuum. Such a deficit has rarely, if ever, been claimed for traditional luminous broad-line objects, i.e., Seyfert 1s and quasars. Indeed, the excess of available continuum to lines is often used to deduce that the BLR covering factor is much 
less than 1 (Yee 1980; Shuder 1981). One exception is Filippenko (1985), who argued that extrapolating the optical continuum of the broad line radio galaxy Pictor A yields a deficit of a factor three. More recently, Barth et al. (1996) perform a similar calculation for the very low luminosity broad line object NGC 4579, which also has a steep UV continuum. They find that the extrapolated ionising continuum is marginally sufficient to explain the observed narrow lines, but fails to explain broad $\mathrm{H} \alpha$ by a substantial factor.

Obviously, if the ionising continuum cannot explain the observed broad emission-lines, then this is a potentially important result and may imply that the continuum is anisotropic, or that an extra heating source is required for Balmer lines in AGN, such as mechanical heating of some kind. However, given that $\mathrm{H} \alpha$ varies, that the UV very likely also varies, and that there is no simultaneous optical and UV data, it is not yet possible to make such a bold statement. Further monitoring, especially in the UV, is very important.

\subsubsection{Black hole mass in NGC 4395}

It is possible to crudely estimate the bolometric luminosity of NGC 4395 by assuming a simplified shape for the whole spectral energy distribution. Long wavelength observations help constrain the SED below $1 \mu \mathrm{m}$. Radio VLA observations of a compact source coincident with the nucleus of NGC 4395 give a flux of $1.24 \pm 0.07 \mathrm{mJy}$ at $20 \mathrm{~cm}$ and $0.56 \pm 0.12$ mJy at $6 \mathrm{~cm}$. IRAS observations at $12 \mu \mathrm{m}, 25 \mu \mathrm{m}$ and $60 \mu \mathrm{m}$ show cirrus-like emission, most probably coming from cold dust heated by the interstellar radiation field within the NGC 4395 disk, rather than warmer dust heated by the nuclear source.

Without far-infrared observations of the nucleus to constrain the bolometric luminosity, two very simple models will be assumed: (1) a power law with index $\alpha=1.7$, normalised at $6800 \AA$ to match the optical spectroscopy, and with cut-offs at $20 \mu \mathrm{m}$ and $2 \mathrm{keV} ;(2)$ the same, but assuming $\alpha=1$. The bolometric luminosities are found to be $L_{B o l}^{(1)}=1.18 \times 10^{41}$ ergs s${ }^{-1}$ for the first case and $L_{B o l}^{(2)}=1.21 \times 10^{41} \mathrm{ergs} \mathrm{s}^{-1}$ for the second. The two values are fortuitously similar, hiding the fact that in the first case nearly all the luminosity is in the mid-IR, and in the second case in the UV. If $100 \mu \mathrm{m}$ had been chosen as the cut off in the far infra-red, the bolometric luminosity would have increased by almost an order of magnitude for case (1), while remaining almost unchanged for case (2). Note also that if the infrared emission is largely reprocessed energy, then some source geometries will include both the raw and reprocessed components and may overestimate the nuclear luminosity. A value of $L_{B o l}=1.2 \times 10^{41} \mathrm{ergs} \mathrm{s}^{-1}$ will be adopted as a representative value for the bolometric luminosity. 
If $v$ is the velocity dispersion of virialized clouds within a distance $R$ of the nucleus then the central mass of an AGN is $M=R v^{2} / G$. While $v$ can be estimated from the full width zero intensity of the broad line components $(v \sim \sqrt{3} / 2 \mathrm{FWZI}(\mathrm{H} \beta)$, Wandel 1991), the value of $R$ is less certain.

In the last 10 years reverberation mapping has made it possible to estimate the size of the BLR in a direct way, in contrast with previous estimations through AGN standard photoionization models which had overestimated $R$ by about an order of magnitude (Peterson 1994; Maoz 1994). Reverberation mapping also appears to confirm a luminosity-size-mass relationship consistent with $R \propto L^{1 / 2}$ (Kaspi et al. 1996). Such a relationship is theoretically expected given two somewhat naïve assumptions: (1) the shape of the ionising continuum is the same for all AGN, and (2) the BLR is characterized by a constant value for the product of the ionising parameter $\mathrm{U}$ and the cloud density $n_{e}$. The best fit slope from AGN data covering two orders of magnitude in luminosity gives $R=1.4 \times 10^{-24} L_{0.1-1 \mu m}^{1 / 2}$ pc, where $L_{0.1-1 \mu m}$ is the $0.1-1 \mu \mathrm{m}$ luminosity in units of ergs s${ }^{-1}$ (Netzer \& Peterson 1997). However, the emission spectra of LINERs have been explained with a value for the ionization parameter 10 times smaller than that inferred for Seyfert galaxies (Ferland \& Netzer 1983). Even if all Seyfert 1 galaxies (including NGC 4395) can be characterised by a particular photoionization regime, any extrapolation of the physical conditions derived for the more luminous objects to very low luminosity sources may introduce major errors in these calculations.

Bearing in mind all the limitations discussed above it is found from the spectra of NGC 4395, that $L_{0.1-1 \mu m} \sim 1.7 \times 10^{40} \mathrm{ergs} \mathrm{s}^{-1}$, implying $R=5.7 \times 10^{14} \mathrm{~cm}$. For FWZI $(\mathrm{H} \beta) \sim 4100$ $\mathrm{km} \mathrm{s}^{-1}$ the mass of the central object in NGC 4395 is then $\sim 1.1 \times 10^{36} \mathrm{~kg}$, or $5.4 \times 10^{5}$ $M_{\odot}$. The corresponding Eddington luminosity is $L_{E d d}=7.0 \times 10^{43} \mathrm{ergs} \mathrm{s}^{-1}$. From the estimates of $M$ and the bolometric luminosity $L_{B o l}$ it is found that the central source is emitting at $\sim 1.7 \times 10^{-3} L_{E d d}$.

Otherwise, if the distance to the BLR does not scale as $L^{1 / 2}$ to very low luminosities but, instead, approaches a minimum value $\left(R_{\min }\right)$, then the central mass in NGC 4395 could be much larger. The smallest observed values of $R$ correspond to $0-3$ light-days for NGC 4151 (Kaspi et al. 1996) and 4 light-days for NGC 4593 (Dietrich et al. 1994). A $R_{\min } \sim 1$ light-day would imply a black hole mass of $2.5 \times 10^{6} M_{\odot}$ and NGC 4395 would be extremely underluminous, emitting at just $\sim 4 \times 10^{-4} L_{E d d}$. 


\subsubsection{X-ray loudness}

To compare the X-ray flux with continuum emission at other wavelengths the spectral indices $\alpha_{o x}$ and $\alpha_{i x}$ will be used, where the index $\alpha_{o x}$ is defined between $2500 \AA(\log \nu=$ $15.08)$ and $2 \mathrm{keV}(\log \nu=17.68)$, and $\alpha_{i x}$ between $1 \mu \mathrm{m}(\log \nu=14.48)$ and $2 \mathrm{keV}$. Using the observed flux at $2500 \AA$, and extrapolating the optical spectroscopy to obtain $F_{1 \mu m}$ it is found that the range of observed X-ray fluxes gives $\alpha_{o x}=2.03-1.76$ and $\alpha_{i x}=1.85-1.63$, where the monochromatic X-ray fluxes at $2 \mathrm{keV}$ have been determined from the broad-band observations assuming a power law index of 1.5 between 0.1 and 2.4 $\mathrm{keV}$.

Values of $\alpha_{o x}$ for AGN (Mushotzky \& Wandel 1989; Laor et al. 1994; Laor et al. 1997; Walter \& Fink 1993; Barth et al. 1996; Mushotzky 1993) are normally regarded as a good estimate of the size of the 'Blue Bump' relative to the X-ray emission. Typically, for quasars and Seyfert 1 galaxies, $\alpha_{o x} \sim 1.5$. For LINERs a much smaller value is found $\left(\alpha_{o x} \sim 0.9\right)$ due to the lower UV fluxes relative to X-rays. NGC 4395 has a steep $\alpha_{o x}$ which is the result of the low X-ray flux rather than powerful UV emission. A similar result is found amongst other low luminosity Seyfert galaxies (Koratkar et al. 1995) which are faint in the UV, but even less bright in the X-rays, giving $\alpha_{o x} \sim 1.6$.

The power law index $\alpha_{i x}$ better characterizes X-ray loudness, since the flux at $1 \mu \mathrm{m}$ is less affected by either internal absorption or the intrinsic magnitude of the UV power. The value of $\alpha_{i x}$ found for NGC $4395(\sim 1.7)$ is much larger than that found for more luminous radio-quiet AGN ( 1.3, Lawrence et al. 1997; see Mushotzky \& Wandel 1989 for a power law slope of 1.27 between $7500 \AA$ and $2 \mathrm{keV}$ ). This shows that the overall spectral shape for NGC 4395 drops rapidly from the optical to the soft X-rays.

Assuming a mean $\alpha_{i x}=1.74$ it is found that NGC 4395 is about 25 times less X-ray loud than more powerful radio-quiet AGN. Adopting an absorption cross section per hydrogen atom at $1 \mathrm{keV}$ of $\sim 2 \times 10^{-22} \mathrm{~cm}^{2}$ (Morrison \& McCammon 1983) it is found that a hydrogen column density of $\sim 1.4 \times 10^{22} \mathrm{~cm}^{-2}$ is required to explain the X-ray deficit. A normal

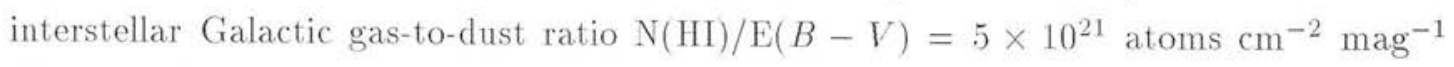
(Sauvage \& Vigroux 1991), and a Galactic extinction law, gives $A_{V} \sim 8.4$ mag. This is not supported by the narrow components of the Balmer lines (see table 4.2) that show a Balmer decrement of $\mathrm{H} \alpha_{N} / \mathrm{H} \beta_{N} \sim 2.5$. However, it is quite usual that local Seyferts with large $\mathrm{X}$-ray columns do not have the corresponding line reddening, suggesting absorption by dust free gas. Examples of X-ray quiet quasars can be found in Laor et al. (1997). It is unknown if these objects are intrinsically faint, or absorbed but otherwise normal quasars. 
However, the observed Balmer decrement of the broad component, $\left\langle\mathrm{H} \alpha_{B} / \mathrm{H} \beta_{B}\right\rangle \sim 6$, does imply an $A_{V} \gtrsim 1$ if an extinction free ratio of 4.5 is adopted as typical for AGN (Osterbrock 1989). This, together with the lack of ionizing flux noted in the previous section and the break of the UV continuum at $\sim 2200 \AA$ could imply that some absorption and extinction occurs in the line of sight towards the central source.

\subsubsection{Comparison with other low luminosity AGN}

The LINER nucleus in M 81 (NGC 3031) is a very well studied low luminosity AGN. Compact X-ray and radio sources coincident with the nucleus have been detected (Fabbiano 1988a; Bartel et al. 1982). It has a broad $\mathrm{H} \alpha$ component with a flux 20 times fainter than the flux detected in NGC 4051, the dimmest 'classical' Seyfert galaxy (Filippenko \& Sargent 1988). Compared to M81, the luminosity of the mean $\mathrm{H} \alpha$ broad component for NGC 4395, taken from table 4.3, is $\lesssim 10$ times smaller. Another example of a LINER nucleus that shows AGN-like properties (broad $\mathrm{H} \alpha$ and X-ray and UV variability) is NGC 4579 (Barth et al. 1996).

Estimates for M 81 show that if photoionization is responsible for its LINER activity, values of $\mathrm{U}=10^{-2.8}$ and $n=10^{9} \mathrm{~cm}^{-3}$ are required, and a radius for the BLR of $1.2 \times 10^{17}$ cm can be inferred (Ho, Filippenko \& Sargent 1996). For NGC 4395 the size of the BLR found in section 4.4.5 is $\sim 200$ times smaller (under the assumption that $\mathrm{U}$ and $n$ are as inferred from more powerful AGN). As has been suggested before, the low ionization lines observed in LINERs could be caused by small values of $U$ and the consequent reduction of the ionizing photon flux, probably as a result of a larger distance between the central source and the emitting clouds (Halpern \& Steiner 1983; Ferland \& Netzer 1983). At the same time the central source in M81 is emitting at $\sim 0.06 \pm 0.04$ per cent of the Eddington limit (Ho, Filippenko \& Sargent 1996), a factor 3 smaller than the value found for NGC 4395.

The shape of the optical featureless continuum during the low state of NGC 4395 resembles the steep UV continuum observed in M 81 (Ho, Filippenko \& Sargent 1996) for which a spectral index close to 2 was derived. Unfortunately, the shape of the continuum of M 81 in the optical range is not well known because of the heavy contamination by starlight.

Optical variability has not been reported for M 81 (Ho, Filippenko \& Sargent 1996). The $\mathrm{H} \alpha$ light-curve shows only slight variation $(10 \%-15 \%)$, the reality of which is unclear, given the complexity of the observations. There is no report of continuum flux changes either. In this respect, the UV variability reported for NGC 4579 could be a further link 
between LINER nuclei and more powerful AGN. X-ray long term variability by significant factors has been reported for M 81 (Petre et al. 1993; Ishisaki et al. 1996) as well as for NGC 4579 (Serlemitsos, Ptak \& Yaqoob 1996).

A recent compilation of spectral energy distributions for a small sample of low-luminosity AGN (including M 81) shows that these objects can be characterised by a steep $(\alpha \sim 1.8)$ optical-UV slope, a radio strength typical for radio-loud AGN, and the lack of a big blue bump (Ho 1999). NGC4395 does not seem to share these traits. The optical to UV distribution resembles the steep UV continuum observed in the sample of low-luminosity AGN only during the low state, but greatly departs from it during the medium and high states. The measured radio flux qualifies NGC 4395 as a radio-quiet object: the parameter $R \equiv S_{\nu}(6 \mathrm{~cm}) / F_{\nu}(B)$ adopts values of $0.1-1.0$ for radio-quiet objects and $>100$ for radioloud ones (Ho 1999); for NGC 4395 it is $0.56 \pm 0.12 / 0.56 \pm 0.28$ (in m.Jy) $=1.0 \pm 0.54$, where the error in the radio flux is given by the uncertainty of the measurement (Sramek 1992), and the error in the $B$-band flux reflects the variability discussed in section 4.3.3. Finally, as in the SEDs presented by Ho (1999), no big blue bump is observed in the energy distribution of NGC 4395. In this case however, the disk emission thought to be responsible for the presence of this feature would be hotter, and therefore shifted towards shorter wavelengths, given the low estimates for the central black hole mass $\left(T_{\text {eff }} \propto M_{B H}^{-1 / 4}\right)$. In NGC 4395 the big blue bump could escape detection, hidden in the far UV.

Is NGC 4395 really the least luminous AGN? The search for 'dwarf' Seyfert nuclei in the Palomar sample carried out by Ho, Filippenko \& Sargent (1997c; 1995b) shows that the luminosity distrubution of the $\mathrm{H} \alpha$ narrow component for the objects in the sample appears to be extremely similar for Seyfert and LINER nuclei, both having a median of $\sim 6 \times 10^{38}$ ergs s$^{-1}$ (Ho 1996). Visual inspection of figure 5 in Ho (1996) shows, however, that some LINERs have luminosities $L\left(H \alpha_{N}\right)<10^{37} \mathrm{ergs} \mathrm{s}^{-1}$, while Seyfert nuclei do not show such low luminosities.

The uncertainty in the true nature of LINER nuclei implies that a more reliable comparison can be made by using only the Type-1 population from the Palomar sample (see discussions in sections 1.1.1 and 5.1). Ho, Filippenko \& Sargent (1997c) found that of the nuclei exhibiting broad $\mathrm{H} \alpha$ more than half were LINERs. The 6 least luminous objects $\left(L\left(H \alpha_{B}\right)<10^{39} \mathrm{ergs} \mathrm{s}^{-1}\right.$, ignoring ambigous spectral classifications and uncertain detenctions of the broad component - see notes to table 1 in Ho, Filippenko \& Sargent 1997c) correspond to 4 LINER and 2 Seyfert nuclei. NGC 4395 is then the least luminous Type-1 object for the distances adopted in the Palomar sample, but two Type-1 LINER nuclei would rival this status if a $5.2 \mathrm{Mpc}$ distance for NGC 4395 (the value used in this work) 
is adopted.

\subsubsection{An advective-dominated accretion flow in NGC 4395}

One possibility to explain the low luminosity for NGC 4395 is the presence of an advectivedominated accretion flow, or ADAF (e.g., Narayan \& Yi 1995; Narayan, Mahadevan \& Quataert 1999). Such a disc is optically thin and cooled by radial advection of heat rather than radiation. Such discs differ from the optically thick variety in several ways. ADAFs have a low radiative efficiency, are thermally and viscously stable and have a vertical thickness similar to their radius (i.e., the geometrically thin approximation does not hold). The combination of an outer, optically thick disk with an inner ADAF seems capable of explaining some apparently under-luminous, hot black-hole binary systems (e.g., Esin, McClintock \& Narayan 1997), although it is unclear physically how to join the two parts of the disc. ADAFs have also been proposed to explain under-luminous galactic nuclei, most notably for Sagittarius A* (Narayan et al. 1998). For NGC 4395 an ADAF could allow for a 'normal' mass black-hole while maintaining consistency with the bolometric luminosity. However, the estimation of the black-hole mass given in section 4.4.5 implies a radiative efficiency higher than that usually associated with ADAFs. For an upper limit for the mass of the black-hole see also Ho (1998).

It has been claimed that dwarf AGN vary considerably less than luminous Seyferts, both in the X-rays (Ptak et al. 1998) and the optical (Shields \& Filippenko 1992; Ho, Filippenko \& Sargent 1996). Ptak et al. (1998) propose that this is because dwarf AGN are ADAFs, and have characteristically larger X-ray emitting regions. However, in NGC 4395 clear large amplitude variability, quite consistent with more luminous Seyferts, has been detected. Perhaps typical dwarf AGN have large black holes together with low accretion rates and advection dominated flows, whereas NGC 4395 might have a small black hole and a higher accretion rate.

\subsubsection{Constraints on nuclear starburst models}

A plausible model for the IR, optical, UV and at least part of the X-ray emission in low luminosity AGN is the starburst model developed by Terlevich and collaborators (see, e.g., Terlevich et al. 1992). NGC 4395 offers a unique opportunity to test this paradigm: with a blue nuclear absolute magnitude of $\sim-10$, a single compact supernova remnant is enough to account for its luminosity. Significant constraints can be obtained from the spectroscopy, and from both the short and long term observed variability. 
In the starburst model, Type-1 AGN correspond to a phase in the evolution of a metal rich star cluster with an age around 10-20 Myr, where the luminosity is dominated by Type II supernovae which are exploding within a dense medium. These compact supernova remnants (cSNR) evolve rapidly and so are highly luminous. The energy output is mostly in the extreme UV and X-ray region of the spectrum. The optical continuum is a mixture of evolved stars and emission from the SNRs, but fast moving fragments are also expected to produce the BLR line emission. Indeed, the spectra of at least some luminous SN exploding in HII regions have a striking resemblance to that of the BLR of Seyfert galaxies (Filippenko 1989). In some well documented cases cSNR radiate about $10^{51}$ ergs in under two years, thus reaching peak luminosities well in excess of $10^{43} \mathrm{ergs} \mathrm{s}^{-1}$ (Aretxaga et al. in preparation).

The discovery of CaIIK absorption in NGC 4395 without a corresponding $4000 \AA$ break, is strong evidence for a young stellar population, as discussed in section 4.3.2. However the observed variability rules out the possibility that the blue light is simply young stars. It is conceivable that the optical continuum is a mixture of early-type stars and variable cSNR emission (or, of course, a mixture of early-type stars and variable accretion disc emission). As the CaIIK equivalent width is a sensitive function of stellar type, the dilution required to match the observed $1 \AA$ equivalent width depends on the age of the cluster. It would be important to test the predictions of such mixture models explicitly against the whole spectrum, but this is not attempted here.

A significant constraint on starburst explanations of NGC 4395 is the long term stability. Although variability by a factor of 2 has been detected on relatively short timescales, comparison with the Filippenko \& Sargent (1989) observations, and indeed with the POSS image discussed by Filippenko, Ho \& Sargent (1993), shows that there has been no significant long term change in mean brightness beyond a factor of 2 in the last $\sim 40$ years. The secular variability in the starburst model comes from the evolution of cSNRs. In more luminous Seyferts, the superposition of cSNR events at a rate governed by the total mass of the starburst could potentially explain the characteristic month-timescale flaring.

In the case of NGC,4395, the observations should correspond to the decline of a single cSNR whose secular blue light-curve is given by $L_{B} \propto t^{-11 / 7}$ (Aretxaga \& Terlevich 1994; Aretxaga, Cid Fernandes \& Terlevich 1997). The peak luminosity of the remnant will be a function of the circumstellar density $\left(L_{B}^{\text {peak }} \propto n^{3 / 4}\right)$, with more luminous events occurring in denser regions. Notice that the observed absolute magnitude of NGC $4395(\sim-10)$ is much fainter than the absolute magnitude of a single cSNR near peak $(\sim-20$ and -18.5 for $n \sim 10^{7}$ and $10^{6} \mathrm{~cm}^{-3}$, respectively). This, together with the low rate of change seen 
in the last 40 years, as mentioned above, suggests that the observation correspond to the late evolution of the cSNR. Using the $t^{-11 / \tau}$ law it is found that a cSNR of age $\sim 300$ years old evolving in a medium with density $n \sim 10^{7} \mathrm{~cm}^{-3}$ would have shown a decline of 10 magnitudes since maximum and of just 0.2 magnitudes in the last 40 years. On the other hand, with a density of $10^{6} \mathrm{~cm}^{-3}$ a $\sim 500$ year old $\mathrm{cSNR}$ would have declined about 8.5 magnitudes since maximum and about 0.1 magnitudes in the last 40 years. Both cases are in agreement with the observations.

It is also possible to compare predicted line strengths by using the cSNR models of Terlevich (1994). If a significant fraction of the nuclear blue luminosity is due to a starburst, then the predicted SN rate is $4.0 \times 10^{-5} \mathrm{yr}^{-1}$. The nucleus in NGC 4395 has:

$$
\begin{array}{llrl}
L_{X} & \sim 3 \times 10^{38} & \text { ergs s}^{-1} \\
L_{B o l} & \sim 5 \times 10^{40} & \text { ergs s}^{-1} \\
L_{H \alpha} & \sim 5.5 \times 10^{38} & \text { ergs s}^{-1} \\
L_{H \beta} & \sim 8.5 \times 10^{38} & \text { ergs s}^{-1}
\end{array}
$$

and the FWHM of the broad component of the broad lines, while difficult to measure, is about $1000 \mathrm{~km} / \mathrm{sec}$.

From table 1.1 and 1.2 of Terlevich (1994) it is possible to see that this set of parameters corresponds to a cSNR evolving in a medium with $n \sim 10^{7} \mathrm{~cm}^{-3}$ and age equal or slightly larger than the last entry corresponding to about 26 yrs. The predicted values for 26 yrs are :

$$
\begin{array}{llrl}
L_{B o l} & \sim 4 \times 10^{40} & \operatorname{ergs~s}^{-1} \\
L_{H \alpha} & \sim 1.3 \times 10^{39} & & \text { ergs s}^{-1} \\
L_{H \beta} & \sim 2.6 \times 10^{38} & & \text { ergs s}^{-1}
\end{array}
$$

and the FWHM of the broad component about twice the shock velocity or $740 \mathrm{~km} \mathrm{~s}^{-1}$. The line properties are then in poor agreement with a cSNR slightly older than 26 years, much younger than the age derived from the absolute blue magnitude.

The short timescale variability in the starburst model is produced by cooling instabilities in the strongly radiative shock (Terlevich et al. 1995; Plewa 1995) or as the result of fragments or density fluctuations in the ejecta interacting with the outer thin shell (Cid 
Fernandes et al. 1996). These mechanisms are capable of producing strong variability in luminous AGN on timescales from hours to weeks. However it might be expected that such an old remnant would have become relatively stable.

In summary, a single cSNR could explain the observed low luminosity nuclear flux from NGC 4395 if the remnant is some tens to hundreds of years old. However, the observed short term (days to months), large-amplitude, optical and X-ray variability, including changes in optical continuum shape, is in clear disagreement with such a starburst model.

\subsection{Summary}

In this chapter optical broad-band images and spectra as well as ROSAT X-ray data for the nearest, most feeble known Seyfert 1 galaxy NGC 4395 has been presented. The main results can be summarised as follows:

1. The optical continuum has been observed to vary by a factor $\sim 1.3$ at $6800 \AA$ and by a factor of $\sim 2.2$ at $3800 \AA$ in a period of 6 months (between July 1996 and January 1997), becoming bluer when brighter. A power law fit shows that the spectral shape changed from an index $\alpha=1.7$ to $\alpha=1.1$.

2. One week of broad-band monitoring was obtained in June 1996. The $B$ and $I$ bands show $\sim 20 \%$ variability in just 24 hours. The inferred spectral shape is consistent with a power law fit with $\alpha \sim 0$ and implies a change by a factor of $\sim 3$ at $4400 \AA$ in just one month, when compared with the low state spectroscopy from July 1996. This evidence shows that NGC 4395 varies in a much more dramatic way than classic Seyfert galaxies.

4. UV spectroscopy shows a clear hump at $\sim 3000 \AA$ consistent with the small blue bump observed in classic AGN. The extrapolation of the featureless UV continuum to higher frequencies assuming $\alpha=1$ gives just enough ionizing photons to explain the observed $\mathrm{H} \alpha$ fluxes. However a deficit of photons is found when the extrapolation is done assuming a steeper $\alpha=1.7$ power law, as implied by the optical low state spectral shape. Anisotropic emission from the central source and/or a different ionizing mechanism might need to be considered to explain the lack of UV photons.

5. HRI and PSPC ROSAT data show that the flux from a weak source consistent with the position of the nucleus changed by a factor of 2 in 15 days. NGC 4395 appears to be $\sim 26$ times less X-ray loud than classic AGN. The low luminosity can be explained as substantial absorption along the line of sight. Spectral analysis of the PSPC data is 
consistent with some intrinsic absorption.

6. Applying reverberation mapping results from classic AGN to NGC 4395, a radius for the BLR of just $2 \times 10^{-4} \mathrm{pc}$ is inferred. This implies that the central source is emitting at $2 \times 10^{-3} L_{E d d}$. However, these results were calculated by extrapolating the observed properties of AGN $\gtrsim 10^{4}$ times more luminous than NGC 4395 and, therefore, must be revisited when more observations on very low luminosity AGN become available.

7. The discovery of a weak CaIIK $\lambda 3933$ absorption line ( $\mathrm{EW} \sim 1 \AA$ ) has been reported, suggesting the presence of a young stellar cluster coincident with the nucleus of NGC 4395. The starlight component from the cluster is estimated to be less than $30 \%$ of the total flux at $4000 \AA$. The cluster may be directly observed as a diffuse component in HST optical imaging, suggesting a 10\% contribution. The IR CaII $\lambda \lambda 8498,8542,8662$ triplet is expected to be detectable. Thus high resolution spectroscopy can provide a direct measurement of the dynamical mass of the young cluster.

8. It has been shown that the ADAF and starburst models for low-luminosity AGN are in poor agreement with the variability patters seen in NGC 4395 . 


\section{Chapter 5}

\section{Nuclear emission from a complete sample of nearby galaxies}

In chapters 2 and 3 the results from the nuclear optical spectroscopy and X-ray imaging were presented. A detailed review was given for every galaxy and new and published data were discussed. Here the sample of galaxies will be studied as a whole in an attempt to address some of the issues raised in chapter 1.

The main aim of the project, and this thesis, is to gain some insight into the nature of low luminosity nuclear activity. The questions to answer include: how common are active nuclei in small galaxies; do they exist at all; is there a minimum size for a galaxy that harbours an AGN; does the AGN luminosity correlate with the host luminosity? In this chapter the optical spectroscopy and X-ray nuclear emission presented earlier will be used to try and answer some of these questions.

\subsection{Optical classification of emission line spectra}

AGN can be recognised by their spectra. Based on the characteristics of their emission lines, AGN are normally classified in two major groups. 'Type-1' objects have two superposed sets of lines: one set of 'narrow lines' with typical electron densities of $n_{e} \sim 10^{3}-10^{6}$ $\mathrm{cm}^{-3}$ and FHWMs $\lesssim 500 \mathrm{~km} \mathrm{~s}^{-1}$, and a second set of 'broad lines' characterised by $n_{e} \sim 10^{9} \mathrm{~cm}^{-3}$ and FHWMs $\lesssim 1000-10000 \mathrm{~km} \mathrm{~s}^{-1}$. 'Type-2' objects differ from 'Type-1' objects in that they only show narrow emission lines. The AGN narrow emission spectra are distinguishable from HII region spectra by the presence of 'harder' ionising photons and therefore a wider range in ionization species. 
LINERs are, arguably, considered members of the AGN family. Their spectra are characterised by emission lines from low ionization species which are distinctly different from both HII regions and classical active nuclei. The idea that LINERs are the low-luminosity tail of the AGN distribution has been challenged by many nuclei with clear Seyfert characteristics but fainter line luminosities than some LINERs. One of the strongest arguments in support of the classification of LINERs as genuine active nuclei is that they also show the 'Type-1'/'Type-2' dichotomy (Ho, Filippenko \& Sargent 1997d). However, it is also clear that the spectra of many objects that fall into the technical definition of a LINER could, in fact, be of stellar origin (Maoz et al. 1998).

The search for low luminosity AGN in the volume-limited sample of galaxies will use the nuclear optical spectroscopy obtained for each galaxy. Signatures of AGN emission will be sought and objects classified accordingly.

As has been described earlier, a list of nuclear targets was constructed for each object after careful examination of the radio, near-IR, optical and X-ray images. This approach was especially important in the case of galaxies with ill defined or 'spotty' nuclei. In some objects this appearance could be related to the presence of dust and regions of star formation and the IR broad-band images helped to locate candidates for the real nuclear position. In other cases galaxies did not seem to have a nucleus at all. Optical spectra were obtained for a total of 32 galaxies, 17 of them having spectra from more than one target in the nuclear region. This gives the 59 observations reported in chapter 3.

As explained in chapter 1, Ho, Filippenko \& Sargent (1995b; 1997b) carried out a similar search obtaining nuclear spectra for a complete sample of $\sim 500$ galaxies in the northern hemisphere with $B_{T} \leq-12.5$ magnitudes. To study the nebular spectra of the nuclei and look for low luminosity AGN signatures they took special care in the removal of the starlight contamination using a series of 'template' galaxies with stellar nuclear spectra (Ho, Filippenko \& Sargent 1993). This, and the large number of objects studied, are the two most important advantages of their survey over this work.

The present study involves a much smaller sample, but has some advantages of its own: (1) the broad-band imaging, including X-ray data, provides alternative targets for the spectroscopy; (2) the range of galaxy sizes extends to dwarf objects; (3) optical spectra have a wider wavelength coverage, including [OII] $\lambda 3727$ which defines the LINER group. 


\begin{tabular}{|lrrc|}
\hline \hline Galaxy & \multicolumn{2}{c}{ Galactic Coord. } & $E(B-V)$ \\
\hline NGC 185 & 120.79 & -14.48 & 0.182 \\
NGC 247 & 113.94 & -83.56 & 0.018 \\
NGC 404 & 127.03 & -27.01 & 0.059 \\
Maffei I & 135.84 & -0.57 & 1.085 \\
Maffei II & 136.50 & -0.33 & 2.326 \\
IC 342 & 138.17 & 10.58 & 0.558 \\
NGC 1560 & 138.36 & 16.03 & 0.188 \\
NGC 2366 & 146.43 & 28.53 & 0.036 \\
NGC 2403 & 150.57 & 29.19 & 0.040 \\
NGC 2976 & 143.92 & 40.91 & 0.070 \\
A 0951+68 & 142.49 & 41.29 & 0.072 \\
NGC 3031 & 142.09 & 40.90 & 0.080 \\
UGC 6456 & 127.84 & 37.33 & 0.036 \\
NGC 3738 & 144.56 & 59.31 & 0.010 \\
NGC 4144 & 143.17 & 69.01 & 0.015 \\
NGC 4150 & 190.45 & 80.47 & 0.018 \\
NGC 4236 & 127.41 & 47.36 & 0.015 \\
UGC 7321 & 241.95 & 81.06 & 0.028 \\
NGC 4395 & 162.10 & 81.54 & 0.017 \\
NGC 4605 & 167.37 & 75.49 & 0.022 \\
NGC 4736 & 123.36 & 76.01 & 0.018 \\
NGC 4826 & 315.71 & 84.42 & 0.041 \\
NGC 5204 & 113.50 & 58.01 & 0.012 \\
NGC 5238 & 107.40 & 64.19 & 0.011 \\
NGC 5457 & 102.04 & 59.77 & 0.009 \\
NGC 6503 & 100.57 & 30.64 & 0.032 \\
NGC 6946 & 95.72 & 11.67 & 0.342 \\
\hline \hline
\end{tabular}

Table 5.1: Galactic extinction in the line of sight towards galaxies with measured emission lines. Taken from Schlegel, Finkbeiner \& Davis (1998). 


\begin{tabular}{|c|c|c|c|c|}
\hline Galaxy & No & Targets & HFS? (Which) & HFS Class \\
\hline NGC 185 & 3 & J1 J3 J4 & Yes $(\mathrm{J} 4)$ & S2 \\
\hline $\mathrm{NGC} 247$ & 1 & & Yes & $\mathrm{H}$ \\
\hline NGC 404 & 1 & & Yes & $\mathrm{L} 2$ \\
\hline Maffei I & 1 & & No & \\
\hline Maffei II & 1 & V1 R1 R2 & No & \\
\hline IC 342 & 2 & $\mathrm{~V} 1 \mathrm{~V} 2$ & Yes (V1) & $\mathrm{H}$ \\
\hline NGC 1560 & 1 & & Yes & $\mathrm{H}$ \\
\hline NGC 2366 & 4 & V1 V3 V4 V5 & Yes (V1) & $?$ \\
\hline NGC 2403 & 1 & V1 & Yes & $\mathrm{H}$ \\
\hline NGC 2976 & 2 & $\mathrm{~V} 1 \mathrm{~V} 2$ & Yes (V1) & $\mathrm{H}$ \\
\hline A $0951+68$ & 1 & $\mathrm{~V} 2(\mathrm{~B})$ & No & \\
\hline UGC 6456 & 4 & $\mathrm{~V} 1 \mathrm{~V} 2 \mathrm{~V} 3 \mathrm{~V} 4$ & No & \\
\hline NGC 3738 & 6 & V1 V 2 V 3 V4 V 5 V 6 & Yes (V1) & $\mathrm{H}$ \\
\hline NGC 4144 & 4 & V1 V2 J1 & Yes (V1) & H \\
\hline NGC 4150 & 1 & V1 & Yes & $\mathrm{T} 2$ \\
\hline NGC 4236 & 2 & $\mathrm{~V} 2 \mathrm{~V} 3$ & Yes (Unknown) & \\
\hline UGC 7321 & 1 & & No & \\
\hline NGC 4395 & 1 & & Yes & S1.8 \\
\hline NGC 4605 & 1 & & Yes & H \\
\hline NGC 4736 & 1 & & Yes & $\mathrm{L} 2$ \\
\hline NGC 4826 & 3 & $\mathrm{~V} 1 \mathrm{R} 1 \mathrm{R} 2$ & Yes (V1) & $\mathrm{T} 2$ \\
\hline NGC 5204 & 2 & V1 X1 & Yes (V1) & $\mathrm{H}$ \\
\hline NGC 5238 & 2 & V1 V2 & No & \\
\hline NGC 5457 & 2 & $\mathrm{~V} 1 \mathrm{~V} 2$ & Yes (V1) & $\mathrm{H}$ \\
\hline NGC 6503 & 1 & & Yes & $\mathrm{T} 2 / \mathrm{S} 2$ \\
\hline NGC 6946 & 1 & & Yes & $\mathrm{H}$ \\
\hline
\end{tabular}

Table 5.2: Journal of galaxies with measured emission lines. The total number and identification of the nuclear targets is given. Observations by Ho, Filippenko \& Sargent (1995b) are also included with their spectral classification $(\mathrm{S}=$ Seyfert, $\mathrm{L}=$ LINER, $\mathrm{T}=$ transition object, $\mathrm{H}=\mathrm{HII}$ region). The number attached to the spectral class gives the Type ('Type-1' or 'Type-2', along with the intermediate Types 1.2, 1.5, 1.8 and 1.9). Although NGC 2366 is present in the spectral atlas by Ho, Filippenko \& Sargent (1995b) it does not appear in the table containing the spectral classifications (Ho, Filippenko \& Sargent 1997b). 


\subsubsection{Diagnostic diagrams}

The two main mechanisms thought to be responsible for the formation of nebular emission lines are photoionisation by UV photons and ionisation by shocks. In the first case, the source of the UV radiation can be divided into two types: thermal photons coming from hot massive young stars, and 'harder' photons coming from a non-thermal source, normally thought to be an active nucleus. The second case, shock heated ionisation, is normally associated with winds and supernova explosions.

Apart from the nature of the photoionising source, which ultimately determines the spectral distribution of UV photons, several other physical factors could play a role in determining the final appearance of the nebular emission: metallicity; density and filling factor of the gas; power and distance of the source; age of the stellar cluster; geometry; etc. Some of these quantities are combined into a single value, called the ionisation parameter $\mathrm{U}$, which measures the ratio between the flux of ionising photons at the position of the nebula and the number of gas particles. Various definitions of $U$ can be found in the literature. The one adopted here is given by:

$$
\mathrm{U}=\frac{Q(\mathrm{H})}{4 \pi r^{2} N_{e} c}
$$

where $Q(H)$ is the total number of ionising photons, $r$ is the distance between the ionising source and the emitting clouds, $N_{e}$ is the electron density of the cloud, and $c$ is the speed of light.

A successful method for distinguishing between different ionising mechanisms uses emission line ratios that are sensitive to changes in the spectral shape of the ionising radiation (Baldwin, Phillips \& Terlevich 1981; Veilleux \& Osterbrock 1987). In this way diagnostic diagrams can separate the (narrow) nebular emission into three major groups: HII regions, AGNs and LINERs (see for example figure 1 in Filippenko \& Terlevich 1992). Theoretical photo-ionisation models of hot stars and power-law continua successfully reproduce the line ratios that correspond to loci occupied by HII regions and AGN (Veilleux \& Osterbrock $1987)$.

As mentioned before, the situation seems more complex for the case of LINER nuclei. Heckman described the LINER class as objects with $[\mathrm{OII}] \lambda 3727 /[\mathrm{OHI}] \lambda 5007 \geq 1.0$ and [OI] $\lambda 6300 /[\mathrm{OIII}] \lambda 5007 \geq 1 / 3$. There is much evidence that not all the objects falling into this definition are driven by the same physical processess, but it is not possible, yet, 
to discern between the AGN and the star formation LINER-types solely on their optical spectroscopic characteristics.

Two different diagnostic diagrams were produced using the sample of nearby galaxies. The line fluxes were taken from table 3.6. Since line ratios are relative values an absolute flux calibration of the spectra is not necessary. The ratios were corrected for Galactic extinction using the $E(B-V)$ values given in table 5.1 using the Galactic extinction law derived by Cardelli, Clayton \& Mathis (1989a). NGC 3031 (M 81) was also included in the diagrams as, along with NGC 4395, these are the only two well studied Seyfert galaxies in the sample. The line ratios for NGC 3031 were taken from Ho. Filippenko \& Sargent (1996).

The optical lines most widely used in diagnostic diagrams are narrow components of $\mathrm{H} \alpha$ and $H \beta$, and the forbidden lines of oxygen, nitrogen and sulphur. Unfortunately, for low redshift galaxies, the weak $[\mathrm{OI}] \lambda 6300$ line is very close to (if not coincident with) the strong oxygen sky-line and is therefore extremely difficult to measure. For this reason, this line flux was obtained for just a handful of objects, as can be appreciated in table 3.6. Balmer emission line fluxes considerably reduced by the presence of a stellar absorption component were not used.

The first approach used in the construction of the diagnostic diagrams was to use the spectral classification given by Ho, Filippenko \& Sargent (1997b) for those galaxies common to both surveys. The rest of the observations were labeled as unclassified. Whenever a galaxy had multiple targets (i.e., galaxies for which more than one nuclear spectrum was obtained), care was taken in finding the right identification of the source observed by Ho, Filippenko \& Sargent (1995b). Table 5.2 shows the complete list of galaxies and targets with measured emission lines and gives details of those galaxies observed by Ho, Filippenko \& Sargent (1997b) and their spectral classification (in four groups: Seyferts, LINERs, transition objects and HII regions). They define transition objects as nuclei that show $[\mathrm{OI}] \lambda 6300$ strengths intermediate between LINERs and HII regions and are probably LINERs contaminated by neighboring HII regions.

\section{The $[\mathrm{OII}] \lambda 3727 /[\mathrm{OIII}] \lambda 5007$ vs $[\mathrm{NII}] \lambda 6584 / \mathrm{H} \alpha$ diagram}

Figure 5.1 shows the $[\mathrm{OII}] \lambda 3727 /[\mathrm{OIII}] \lambda 5007 \mathrm{vs}[\mathrm{NII}] \lambda 6584 / \mathrm{H} \alpha$ diagnostic diagram first developed by Baldwin, Phillips \& Terlevich (1981). Filled symbols have been used to show the nuclear spectra classified by Ho, Filippenko \& Sargent (1997b), while empty circles show the unclassified targets. Notice that many galaxies have multiple observations and 

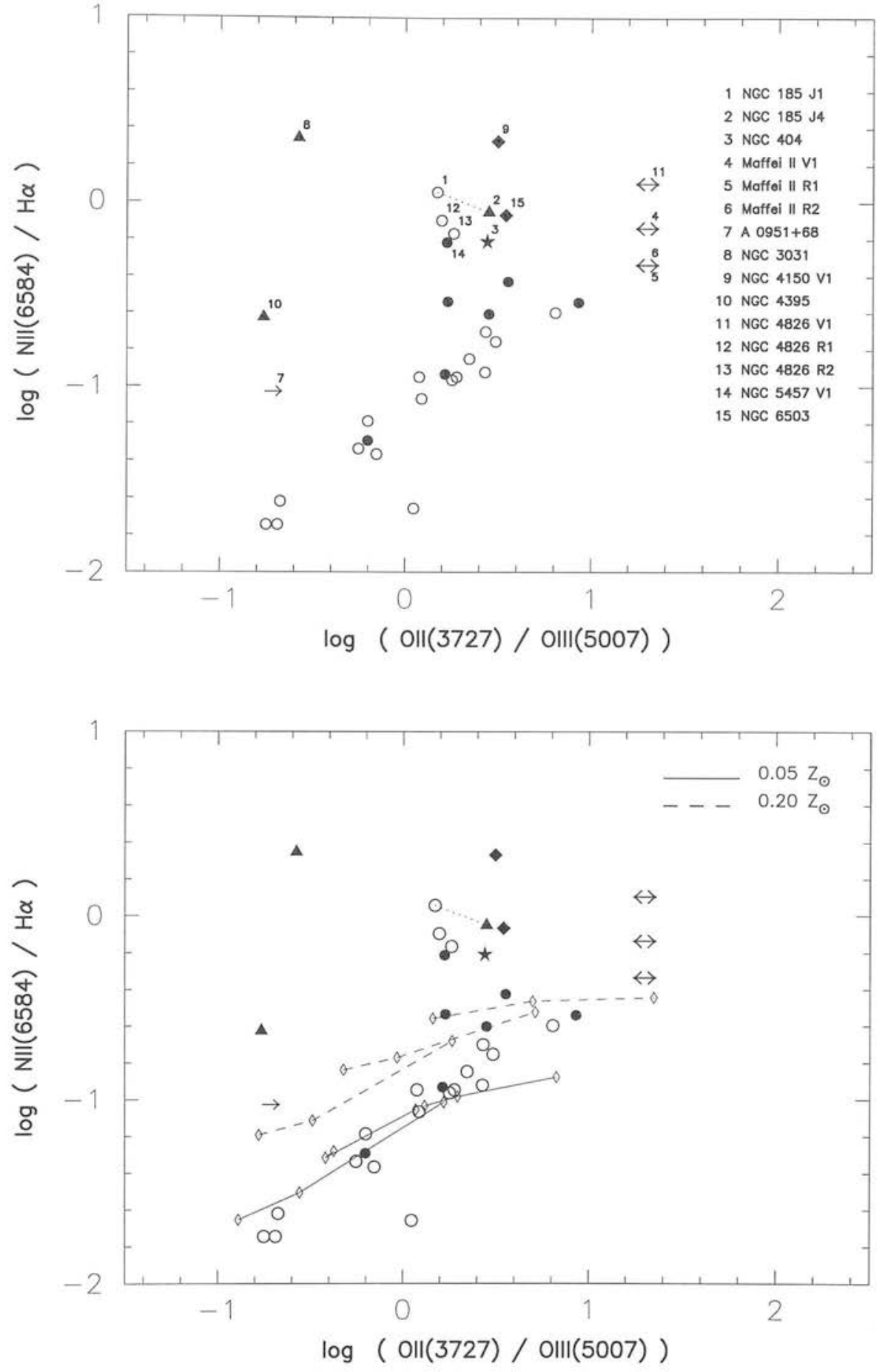

Figure 5.1: [OII] $\lambda 3727 /[\mathrm{OIII}] \lambda 5007$ vs [NII] $\lambda 6584 / \mathrm{H} \alpha \lambda 6503$ diagnostic diagrams. Solid symbols show: HII region nuclei $(\bullet)$, transition objects $(\diamond)$, LINERs $(\star)$, and Seyfert nuclei ( $\mathbf{\Lambda}$ ), as classified by Ho, Filippenko \& Sargent (1997b). Unclassified objects are shown with empty circles. Upper limits are shown with arrows. Objects of interest have been labeled and identified in the upper panel (see also table 5.2). HII region models developed by Garcia-Vargas, Bressan \& Diaz (1995) have been included in the lower panel. Two families of curves are shown with metallicities 0.05 and 0.20 times solar. Each curve represents the evolution of a single star cluster. Diamonds $(\diamond)$ mark the cluster age for 1 , 2 and 3 Myr. The ionisation parameter $U$ varies continously along each curve and from one adjacent curve to another, with limiting values of $\log U=-2.0$ at the left end and $\log \mathrm{U}=-3.3$ at the right. 
therefore several empty circles can belong to the same galaxy. The top panel in figure 5.1 shows that most of the unclassified objects lie in a well defined sequence that crosses the diagram diagonally. Most of the sources classified as HII regions by Ho, Filippenko \& Sargent (1997b) are located at the top of this sequence.

The bottom panel of figure 5.1 includes models of HII region lines ratios taken from GarciaVargas, Bressan \& Diaz (1995) for two different metallicities: $Z / Z_{\odot}=0.05$ (continuus line) and $Z / Z_{\odot}=0.20$ (dashed line). The grids of models follow the evolution in time of the ionising star cluster from 1 to 3 Myr for different cluster sizes and distances between the cluster and the emitting clouds. Variations in these parameters effectively change the value of the ionisation parameter $\mathrm{U}$ and move the predicted position of the line ratios along a rather narrow track on the [OII] $\lambda 3727 /[\mathrm{OIII}] \lambda 5007$ vs [NII] $\lambda 6584 / \mathrm{H} \alpha$ diagram. Variations in the metallicity will shift vertically the position of this track as can be seen in figure 5.1. It is clear that the models can reproduce the loci occupied by the sequence of unclassified objects and by those nuclei classified as HII regions by Ho, Filippenko \& Sargent (1997b) (except for NGC 5457, see below).

The unclassified targets laying along the HII region tracks correspond to spectra obtained for galaxies NGC 2366, A 0951+68, UGC6456, NGC 4236, UGC7321, and NGC5238. Note that NGC 2366, UGC 6456 and NGC 4236 do not have an obvious nucleus. However, it is possible to state that all candidate nuclei are now classified as HII regions. A $0951+68$, labeled as source 7 in figure 5.1 , is very small galaxy $\left(M_{B} \approx-13\right)$ that is actually a pair of close, spheroidal galaxies, each with a distinct nucleus. Spectroscopy of the nuclei showed one to be starlight and the other to have high excitation emission lines ([OIII] $\lambda 5007 / \mathrm{H} \beta \sim 6$ ) consistent with a very low-metallicity nuclear starburst (Johnson et al. 1997).

All objects not located at the HII region locus have been labeled with numbers and identified in the top panel of figure 5.1. All of them, except for the Seyfert galaxies NGC 4395 and NGC 3031, lie on the loci normally ocupied by LINERs.

NGC 5457 is the only nucleus in this LINER group which has been classified as an HII region by Ho, Filippenko \& Sargent (1997b). The [OII] $\lambda 3727 /[\mathrm{OIII}] \lambda 5007$ ratio clearly corresponds to those seen in LINERs. The observed position in the diagram cannot be explained by internal extinction because the [NII] $\lambda 6584 / \mathrm{H} \alpha$ ratio is not sensitive to such corrections (extinction can however, affect the value of the [OII] $\lambda 3727 /[\mathrm{OIII}] \lambda 5007$ ratio). Stellar Balmer absorption could shift the position of the nucleus towards the HII region loci, but (as can be seen from the optical spectra shown in figure 3.27 ) the $\mathrm{H} \alpha$ absorption 
component is probably negligible. The lack of a reliable measurement of the [OI] $\lambda 6300$ line prevents a certain classification of the nucleus as a LINER or a transition object.

The value of the $[\mathrm{NII}] \lambda 6584 / \mathrm{H} \alpha$ ratio for the three spectra obtained for Maffei II are shown to the left of the LINER loci with a double arrow (sources 4, 5, and $6 ; 5$ and 6 are coincident). Since no blue spectra are available for these observations the [OII] $\lambda 3727 /[\mathrm{OIII}] \lambda 5007$ ratios are unknown. A similar argument to that given above can be applied to this object: the $[\mathrm{NII}] \lambda 6584 / \mathrm{H} \alpha$ ratio of source $\mathrm{V} 1$ is compatible with those seen in LINERs and transition objects. However, this classification is very uncertain since no oxygen lines have been observed.

The unclassified targets labeled as 12 and 13 in figure 5.1 correspond to spectra taken at the position of two radio sources $3^{\prime \prime}$ and $4^{\prime \prime}$ away from the LINER nucleus in NGC 4826 . These two spectra also show LINER characteristics. Since the observation of the nucleus of the galaxy is strongly affected by starlight contamination, the line measurements from the data presented in chapter 3 are not shown in figure 5.1. Instead, the [NII] $\lambda 6584 / \mathrm{H} \alpha$ ratio given Ho, Filippenko \& Sargent (1997b) is used and plotted with a double arrow since no [OII] $\lambda 3727$ flux measurement is available. Assuming a single central ionising source, the ratio between the value of the ionising parameter at the position of the nucleus and the position of the radio sources should be $\sim 10\left(\mathrm{U} \propto r^{-2}\right)$. Models show that changes in the value of $U$ by this magnitude will be seen as a considerable reduction of the [OII] $\lambda 3727 /[\mathrm{OIII}] \lambda 5007$ ratio and a smaller reduction of the [NII] $\lambda 6584 / \mathrm{H} \alpha$ ratio (see for example figure 2 in Ferland \& Netzer 1983). This effect is consistent with the observed change in the $[\mathrm{NII}] \lambda 6584 / \mathrm{H} \alpha$ ratio and therefore does not disagree with the possibility of a single central source in the LINER nucleus of NGC 4826. However, to definitely rule out or confirm this hypothesis, the [OII] $\lambda 3727 /[\mathrm{OIII}] \lambda 5007$ must be obtained.

\section{The $[\mathrm{OI}] \lambda 6300 /[\mathrm{OIII}] \lambda 5007$ vs $[\mathrm{OI}] \lambda 3727 /[\mathrm{OIII}] \lambda 5007$ diagram}

Figure 5.2 shows the $[\mathrm{OI}] \lambda 6300 /[\mathrm{OIII}] \lambda 5007$ vs $[\mathrm{OI}] \lambda 3727 /[\mathrm{OIII}] \lambda 5007$ diagnostic diagram. This diagram defines the LINER class according to the criteria adopted by Heckman (1980). The loci of LINERs is shown as a shaded area in the right top corner of the figure. As noted before it has only been possible to measure the [OI] $\lambda 6300$ line for a few galaxies which explains the reduced number of objects shown in this diagram. The convention for the symbols is the same as in figure 5.1. The line ratios of three Seyfert galaxies from the literature have also been included to better define the region occupied for these objects. They are shown with asterisks. 


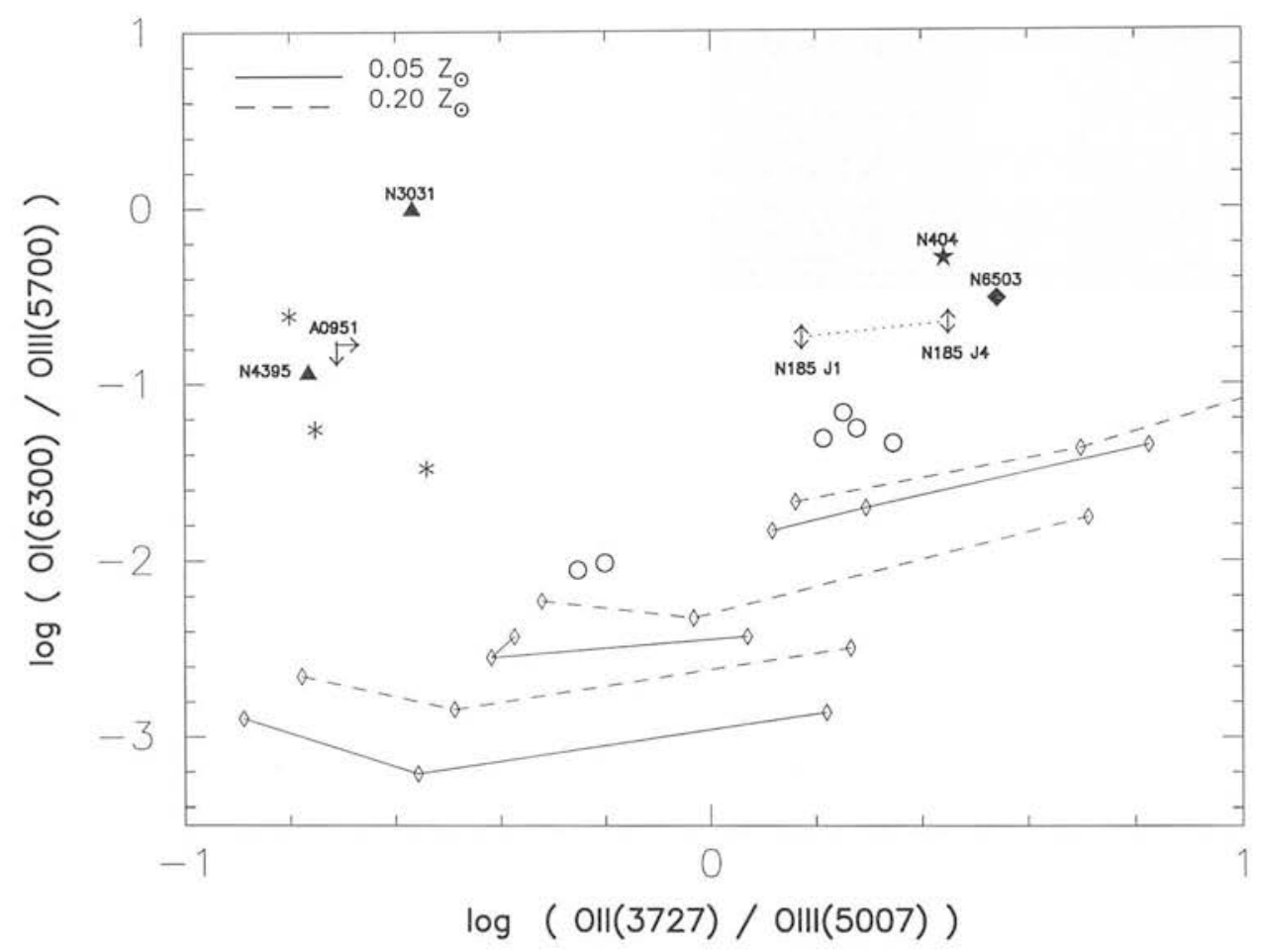

Figure 5.2: [OI] $\lambda 6300 /[\mathrm{OIII}] \lambda 5007$ vs [OII] $\lambda 3727 /[\mathrm{OIII}] \lambda 5007$ diagnostic diagram. Symbols as in figure 5.1. The shaded area represents the LINER loci as defined by Heckman (1980). Three examples of Seyfert nuclei have been added and are shown with asterisks (*). The narrow line ratios were taken from Osterbrock \& Koski (1976) (for NGC 4151), Boksenberg \& Netzer (1977) (for NGC 3516) and from Kunth, Sargent \& Bothun (1987) (for G1200-2038). HII region models are as in figure 5.1. 
The dwarf galaxy NGC 185 was classified as a Seyfert 2 by Ho, Filippenko \& Sargent (1997b) based on their spectrum of the source they identified as the nucleus. Comparison of the spectra shown in chapter 3 with the data published by Ho, Filippenko \& Sargent (1997b) suggests that this probably corresponds to the IR source J4 shown in figure 3.5 .

For two of the four spectra obtained in the nuclear region of the galaxy ( $\mathrm{J} 1$ and J4) emission lines were measured. They are included in figures 5.1 and 5.2 and, in each case, are joined by a dotted line. In figure $5.1 \mathrm{~J} 1$ is shown as an unclassified target and both objects are in clear disagreement with the line ratios expected from HII regions. Ho, Filippenko \& Sargent (1997b) seem to have based the classification of their observations on the $[\mathrm{OIII}] \lambda .5007 / \mathrm{H} \beta$ ratio, which they measured to be 3.32 . The criteria adopted by Ho, Filippenko \& Sargent (1997b), stating that LINER nuclei have [OIII] $\lambda 5007 / \mathrm{H} \beta<3$, while Seyferts have $[\mathrm{OIII}] \lambda 5007 / \mathrm{H} \beta \geq 3$, is not very robust since HII regions like the starburst nucleus in A $0951+68$ can have [OIII] $\lambda 5007 / \mathrm{H} \beta$ as high 6 !

Unfortunately the [OI] $\lambda 6300$ lines are impossible to measure for the NGC 185 sources J1 and J4 due to heavy sky contamination. The OI $\lambda 6300$ values adopted in figure 5.2 correspond to the level of the noise in neighbouring regions, but the real values are unknown and could be either larger or smaller. Despite this disadvantage, it becomes clear from figure 5.2 that the $[\mathrm{OII}] \lambda 3727 /[\mathrm{OIII}] \lambda 5007$ ratio alone is able to distinguish between Seyfert and LINER nuclei, since all Seyferts have [OII] $\lambda 3727 /[\mathrm{OIII}] \lambda 5007<0.6$ while LINERs, by definition, have [OII] $\lambda 3727 /[\mathrm{OIII}] \lambda 5007>1.0$ (a limit of [OII] $\lambda 3727 /[\mathrm{OIII}] \lambda 5007=1$ has been adopted as the boundary between Seyferts and LINERs in figure 1 of Filippenko \& Terlevich (1992)). On these bases J1 and J4 are clearly incompatible with a Seyfert classification and, as discussed above based on the 5.1 diagnostic diagram, also incompatible with being HII regions. It is suggested here that this nuclear region should be classified as a LINER or transition object.

As was mentioned earlier it appears that LINERs are not an homogeneous class of objects. NGC 185 adds support to this idea since at least two distinctive knots of emission in its nuclear region show LINER characteristics. This geometry clashes with the hypothesis of an AGN-like single central ionising source. Instead, the NGC 185 nuclear region seems to break into several stellar clusters exhibiting LINER spectra. The measurement of the $[\mathrm{OI}] \lambda 6300$ lines would have allowed for the comparison of these sources with the models developed by Filippenko \& Terlevich (1992) which postulate that 'weak-[OI] $\lambda 6300$ LINERs' are in fact photoionised by hot early O-type stars with a significantly lower ionisation parameter than normal HII regions. Unfortunately, it is not possible to do so. 


\subsubsection{The final spectral classification}

Table 5.3 summarises the final spectral classifications for all the galaxies with spectral information. New objects or objects misclassified by Ho, Filippenko \& Sargent (1997b) are identified in the notes. On the basis of the discussion above, NGC 185 and NGC 5457 are now classified as tentative Type-2 LINERs or transition objects. The classification of Maffei I as an HII region is solely based on the presence of $\mathrm{H} \alpha$ and the lack of strong nitrogen or sulphur lines. This classification is uncertain. Equally uncertain is the classification of Maffei II as a LINER or transition object, since only the [NII] $\lambda 6584 / \mathrm{H} \alpha$ ratio has been measured. The spectral classification given by Ho, Filippenko \& Sargent (1997b) has been adopted for the LINER NGC 4736 since its spectrum is heavily contaminated by starlight and, therefore, it has not been analysed here. NGC 3031 is a well known Seyfert galaxy (Peimbert \& Torres-Peimbert 1981; Ho, Filippenko \& Sargent 1996) and has been included as such in the table. NGC 253 is an extensively studied starburst galaxy and so has been included as an HII region (Engelbracht et al. 1998; Beck \& Beckwith 1984). Finally, Ho, Filippenko \& Sargent (1997b) show a clear HII region spectrum for NGC 4244 which has, therefore, also been included.

\subsection{The AGN population}

In light of the spectral classification obtained in the previous section for the objects in the volume-limited sample of galaxies it is now possible to establish the demographics of AGN and their relationship with the parent galaxy distribution.

Of the 33 galaxies from the sample which have a spectral classification (Maffei I and Maffei II are not included here because of their uncertain classification) in the previous section: 4 have a stellar spectrum; 20 have HII region nuclei; 5 are Type- 2 transition objects; 2 are Type-2 LINERs; 2 have Type-1 Seyfert nuclei. Note that NGC185, NGC 5457 and NGC 6503 have been taken as transition objects and that the AGN class includes Seyferts, LINERs and transition objects.

The fraction of galaxies with emission lines is very large ( $\sim 90 \%)$, in good agreement with the results found by Ho, Filippenko \& Sargent (1997d). Although the fractions of AGN and Type- 1 objects are lower than in the Palomar sample $(\sim 30 \%$ and $6 \%$ respectively, compared with $\sim 40 \%$ and $\sim 10 \%$ reported by Ho, Filippenko \& Sargent 1997d), the values are consistent with the errors associated with low counts. 


\begin{tabular}{|lccc|}
\hline \hline Galaxy & $-M_{B}$ & Final Class & Notes \\
\hline NGC 147 & 13.71 & $\mathrm{~A}$ & \\
NGC 185 & 13.94 & L2 or T2 & $\mathrm{R}$ \\
NGC 205 & 15.24 & $\mathrm{~A}$ & \\
NGC 221 & 15.06 & $\mathrm{~A}$ & \\
NGC 224 & 19.69 & $\mathrm{~A}$ & \\
NGC 247 & 18.32 & $\mathrm{H}$ & \\
NGC 253 & 20.26 & $\mathrm{H}$ & \\
NGC 404 & 17.07 & $\mathrm{~L} 2$ & \\
NGC 598 & 18.43 & $\mathrm{H}$ & \\
Maffei I & 13.87 & $\mathrm{H}:$ & $\mathrm{N}$ \\
Maffei II & 8.08 & L2/T2: & $\mathrm{N}$ \\
IC 342 & 19.50 & $\mathrm{H}$ & \\
NGC 1560 & 15.95 & $\mathrm{H}$ & \\
NGC 2366 & 17.53 & $\mathrm{H}$ & $\mathrm{N}$ \\
NGC 2403 & 20.25 & $\mathrm{H}$ & \\
NGC 2976 & 16.98 & $\mathrm{H}$ & \\
A 0951+68 & 11.21 & $\mathrm{H}$ & $\mathrm{N}$ \\
NGC 3031 & 19.22 & $\mathrm{~S} 1.5$ & $\mathrm{H}$ \\
UGC 6456 & 12.19 & $\mathrm{H}$ & $\mathrm{N}$ \\
NGC 3738 & 16.78 & $\mathrm{H}$ & \\
NGC 4136 & 17.63 & $\mathrm{H}$ & $\mathrm{H}$ \\
NGC 4144 & 16.83 & $\mathrm{H}$ & \\
NGC 4150 & 15.30 & $\mathrm{~T} 2$ & $\mathrm{H}$ \\
NGC 4236 & 17.50 & $\mathrm{H}$ & $\mathrm{N}$ \\
NGC 4244 & 17.59 & $\mathrm{H}$ & $\mathrm{H}$ \\
UGC 7321 & 14.82 & $\mathrm{H}$ & $\mathrm{N}$ \\
NGC 4395 & 17.89 & $\mathrm{~S} 1.8$ & \\
NGC 4605 & 17.80 & $\mathrm{H}$ & \\
NGC 4736 & 20.00 & $\mathrm{~L} 2$ & $\mathrm{H}$ \\
NGC 4826 & 19.39 & $\mathrm{~T} 2$ & \\
NGC 5204 & 17.39 & $\mathrm{H}$ & \\
NGC 5238 & 15.79 & $\mathrm{H}$ & $\mathrm{N}$ \\
NGC 5457 & 21.22 & $\mathrm{H} 2 / \mathrm{T} 2$ & $\mathrm{R}$ \\
NGC 6503 & 18.28 & $\mathrm{~T} 2 / \mathrm{S} 2$ & \\
NGC 6946 & 19.66 & & \\
\hline \hline
\end{tabular}

Table 5.3: Final spectral classification for the galaxies in the volume-limited sample. The different classes are denoted as $\mathrm{A}=$ starlight dominated nuclei, $\mathrm{S}=$ Seyfert, $\mathrm{L}=\mathrm{LINER}$, $\mathrm{T}=$ transition object, and $\mathrm{H}=\mathrm{HII}$ region. Objects with highly uncertain classifications are shown with a ' $\because$. Objects that have been reclassified are shown with an ' $R$ '. New classifications are shown with a ' $\mathrm{N}$ ', and objects without spectra and for which the classification given by Ho, Filippenko \& Sargent (1997b) has been adopted are shown with a 'H'. NGC 253 has also been included and classified as a HII region. 
Total $=33 ;$ Seyferts $=2 ;$ LINERs $=2 ;$ Transition Objs $=5$

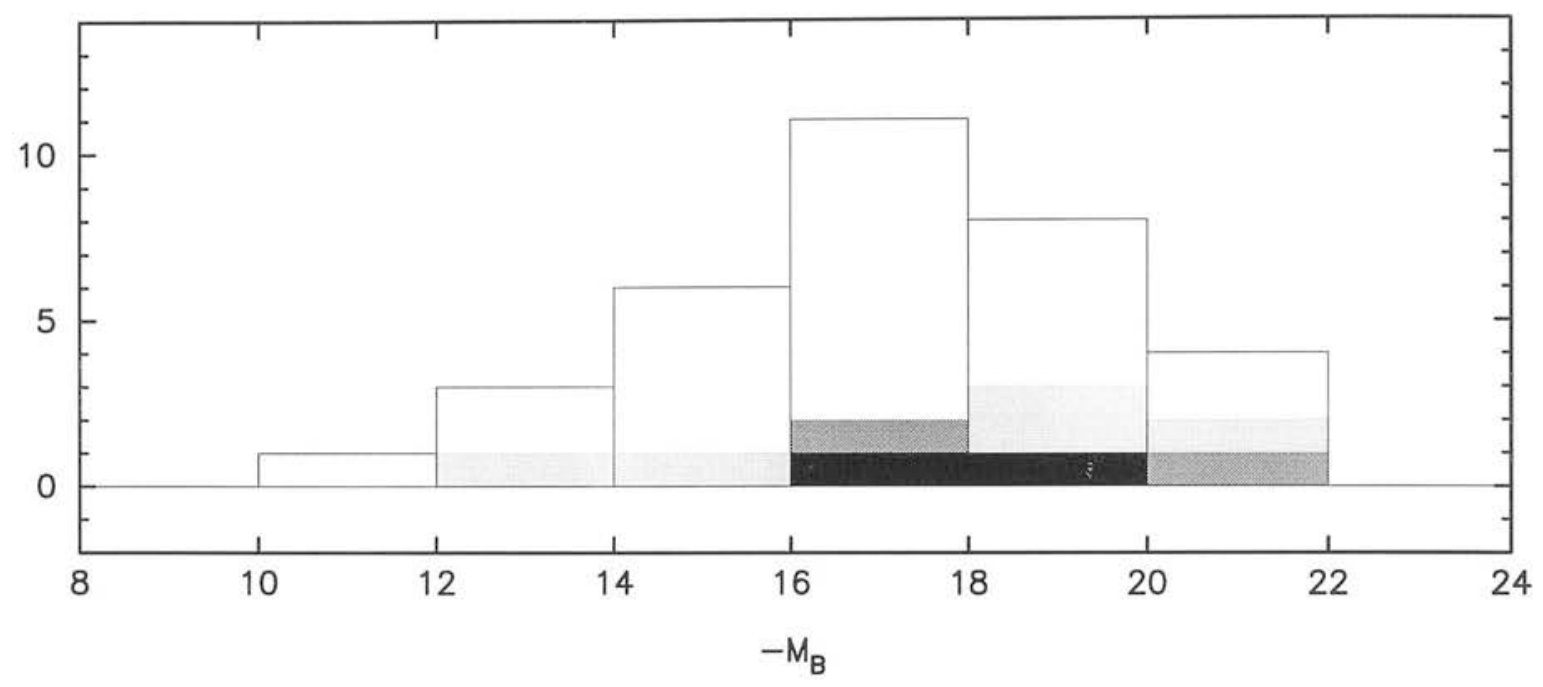

Figure 5.3: Distribution of different spectral groups in a sample of 33 classified objects as a function of the host galaxy absolute magnitude. Different histograms show the whole sample (outer line), Seyfert galaxies (solid black), LINERs (dark grey) and transition objects (light grey).

Assuming a Poisson distribution at a low count level and using equations B.3 and B.4 from appendix B, the number of AGN and Type-1 objects with their respective upper and lower ' $1 \sigma$ ' errors are $9_{-2.9}^{+4.1}$ and $2_{-1.3}^{+2.6}$. To propagate these errors it is necessary to assume that they are Gaussian distributed, and therefore symmetric. To do this the mean of the upper and lower ' $1 \sigma s$ ' will be adopted as representative of a symmetric error distribution. Under this approximation the percentages given above for the detection of AGN and Type-1 objects in the volume limited sample of galaxies becomes $(30 \pm 10) \%$ and $(6 \pm 5) \%$ respectively, which is consistent with the values found by Ho, Filippenko \& Sargent (1997d).

\subsubsection{Comparison with the normal galaxy population}

Do dwarf galaxies have active nuclei? It is possible to address this question by looking at the distribution as a function of absolute magnitude of active and 'normal' galaxies (i.e., those that are not classified as AGN). Figure 5.3 shows the distribution of galaxy luminosities for the population of 24 'normal' galaxies and for the different AGN groups (i.e., Seyferts, LINERs and transition objects).

A Kolmogorov-Smirnov test was used to compare the distribution of 'normal' galaxies 


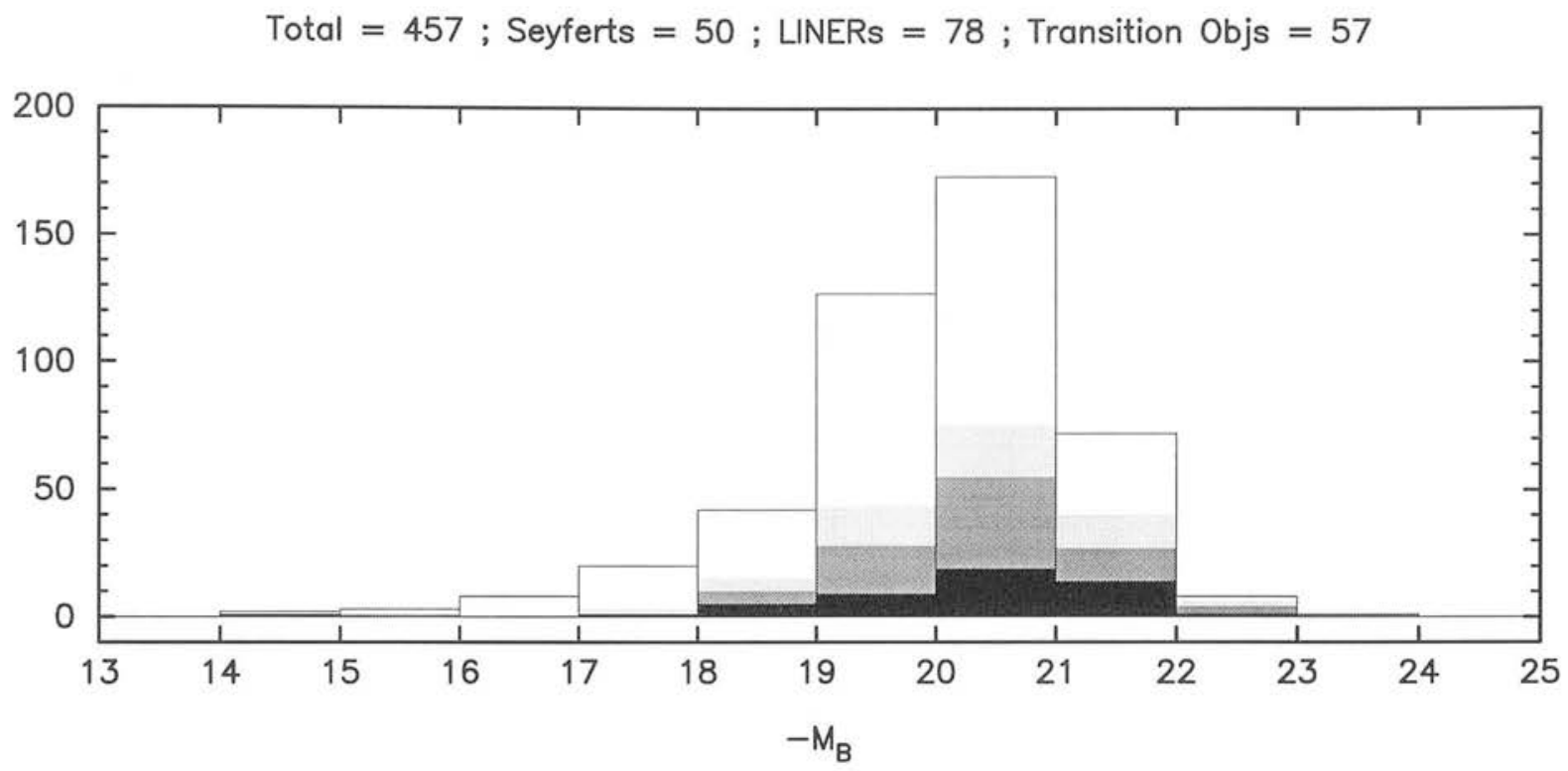

Figure 5.4: Distribution of different spectral groups in the Palomar sample (Ho, Filippenko \& Sargent 1997b) as a function of the host galaxy absolute magnitude. Different histograms show the whole sample (outer line), Seyfert galaxies (solid black), LINERs (dark grey) and transition objects (light grey).

with those classified as AGN (for details see appendix B). Given the low number of data points in the AGN sample, the test shows only marginally significant evidence that the distributions are different $\left(Q(\xi)=1.1 \times 10^{-1}\right)$.

This test can be repeated using the Palomar sample. Objects with highly uncertain classifications (shown with a '::' in table 4 of Ho, Filippenko \& Sargent (1997b)) were not used in the calculations. Whenever objects had a double classification (e.g., S2/L2), the first spectral class was adopted as representative.

Figure 5.4 shows the distribution of normal galaxies and AGNs as function of the host galaxy luminosity for the Palomar sample. The Kolmogorov-Smirnov test shows that the evidence that the populations of 'normal' galaxies and AGNs are distributed differently is highly significant $\left(Q(\xi)=3.7 \times 10^{-4}\right)$. The result is also significant if only Seyfert galaxies are regarded as $\operatorname{AGN}\left(Q(\xi)=1.0 \times 10^{-3}\right)$.

These results imply that the AGN host galaxies show a different distribution to inactive galaxies as a function of luminosity. Visual inspection of the histograms shown in figures 5.3 and 5.4 confirms previous work that claimed that the fraction of AGN to field galaxies decreases with decreasing host luminosity (Huchra \& Burg 1992). The better sensitivity of the present surveys shows, however, that the fraction of AGN to field galaxies had been 


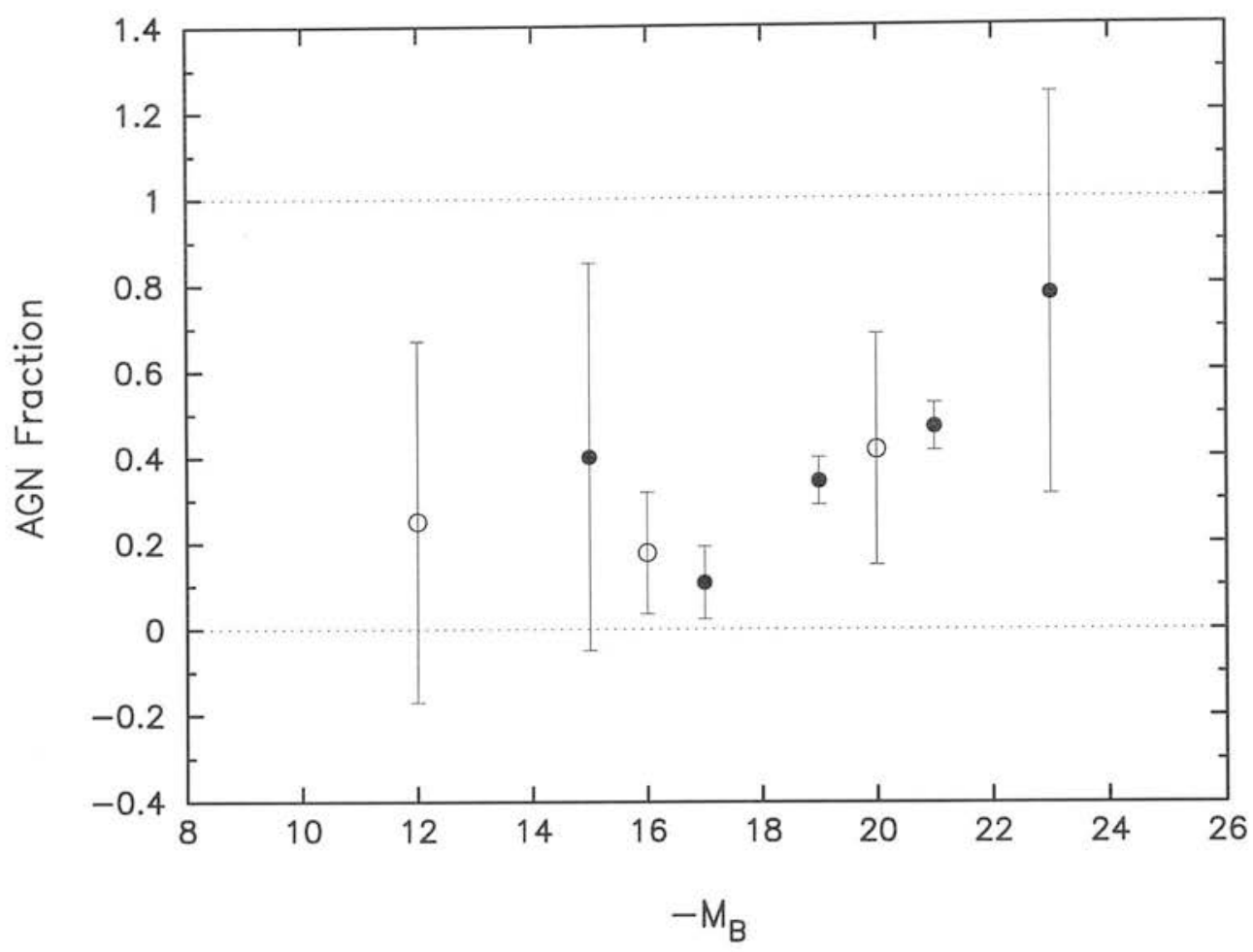

Figure 5.5: Fraction of AGN to total number of galaxies as a function of galaxy luminosity. Results come from the volume-limited sample (o) and the Palomar sample of galaxies $(\bullet)$. The error bars represent $1 \sigma$ confidence levels (see text).

underestimated: in the $-20<M_{B}<-18$ bin the fraction 'AGN/normal' found in the Palomar sample is equal to $\sim 0.35$, while Huchra \& Burg (1992) found a fraction equal to $\sim 0.25$ from the CfA Redshift Survey (both include Seyfert and LINERs as active galaxies). The inclusion of dwarf galaxies in the studies has also extended the low luminosity tail of the distributions by more than 2 orders of magnitude.

\subsubsection{The luminosity dependent fraction of AGN}

It is now possible to study the characteristics of the low luminosity tail of the AGN population. The ratio of AGN to the total number of galaxies (i.e., AGN plus 'normal' galaxies) as a function of galaxy luminosity is shown in figure 5.5. Results from the volumelimited sample of galaxies have been binned into three points and are shown with empty circles. Results from the Palomar sample have been binned into five data-points and are shown with filled circles. As before, errors were computed using equations B.3 and B.4 from appendix B, assuming that the mean between the upper and lower ' $1 \sigma$ 's is Gaussian distributed and, therefore, symmetric (as can be seen in figure 5.5, this approximation sometimes yields error bars which extend meaninglessly below zero or above one). 
A clear trend can be seen in figure 5.5: the fraction of AGN to field galaxies increases with galaxy magnitude. Although errors are large at the low luminosity end, the distribution of the ratios suggests a flattening of the fraction for absolute magnitudes $z-17$. This is a result of detecting two active nuclei in galaxies with absolute magnitudes larger than -17 . However, they correspond to NGC 185 and NGC 404, which, as discussed before, could in fact be powered by star formation and not by active nuclei. If this is the case, the trend seen in figure 5.5 is more suggestive of a lower cutoff for the absolute magnitude of active galaxies around $M_{B} \sim-17$.

It has to be remembered that very small galaxies are still poorly represented in the distributions and that the detection of nuclear activity in these objects is not easy, particularly if the level of activity correlates with galaxy size, as some observations suggest (see section 5.4.1). It is possible that the low luminosity tail of the AGN population would show a very different picture if the study of these galaxies could reach sensitivities of $\sim 10^{36}$ ergs $\mathrm{s}^{-1}$ or less.

\subsection{Nuclear X-ray sources}

A review of the X-ray emission from nearby galaxies was given in chapter 1 . The emission observed from these galaxies is understood to be directly associated with stars and the later stages of stellar evolution. XRBs and SNRs are responsible for the bulk of the Xray luminosity and their properties are reviewed in table 5.4. Although stars and CVs are more numerous they have much lower luminosities: about 10,000 individuals sources would be needed to account for the X-ray emission of a single luminous XRB.

AGN are ubiquitous X-ray emitters. The detection of nuclear X-ray sources, therefore, is a strong hint for the presence of an active nucleus. The 'soft' $\mathrm{X}$-ray spectra of radio quiet AGN is normally well fitted by a power law with index $\alpha=1.15 \pm 0.14$ (Laor et al. 1997). The X-ray continuum accounts for $z 10 \%$ of the bolometric luminosity and is characterised by very large amplitude variability on short time-scales. The amplitude of the variability at a fixed time-scale has been found to correlate inversely with the optical luminosity of the source.

In this section the nuclear X-ray sources found in the volume-limited sample of galaxies will be studied in detail. Correlations with host galaxy absolute magnitudes and emission line luminosities will be analysed and the relative possibilities of stellar and nuclear activity being responsible for the observed emission will be discussed. 


\begin{tabular}{|c|c|c|}
\hline Source & $\begin{array}{c}\log L_{X} \\
\left(\operatorname{ergs~s}^{-1}\right)\end{array}$ & Spectrum \\
\hline \multicolumn{3}{|c|}{ The Galaxy } \\
\hline $\begin{array}{l}\text { Stars } \\
\text { CV } \\
\text { SNR } \\
\text { HMXRB } \\
\text { LMXRB }\end{array}$ & $\begin{array}{l}\lesssim 33 \\
\lesssim 32 \\
\lesssim 36 \\
\lesssim 37 \\
\lesssim 38\end{array}$ & $\begin{array}{l}\text { soft } \\
\text { hard } \\
\text { soft } \\
\text { hard } \\
\text { hard }\end{array}$ \\
\hline \multicolumn{3}{|c|}{ The Magellanic Clouds } \\
\hline All sources & $\begin{array}{l}\text { 35-39 } \\
\text { M } 31\end{array}$ & \\
\hline All sources & $35-38$ & \\
\hline
\end{tabular}

Table 5.4: Average properties of X-ray sources (adapted from Watson (1990) and updated with values taken from Van Paradijs \& McClintock (1995)).

Of the 12 galaxies classified as dwarfs in the sample (see table 1.1), only four have Einstein or ROSAT HRI observations (NGC147, NGC 185, Leo B and UGC 6456), and of these only one has a positive detection of an X-ray source (UGC6456), and even this one is not nuclear. This section will, therefore, not include the dwarf objects in any analysis, unless otherwise indicated.

\subsubsection{X-ray nuclear and host galaxy luminosities}

Table 2.4 in chapter 2 shows that 13 of the 29 galaxies with HRI data have X-ray sources associated with their nuclear regions. This represents a $45 \%$ rate of detection. Figure 5.6 shows the nuclear X-ray luminosities and upper limits for the galaxies in the sample as a function of the blue absolute magnitude of the host. The following interesting results can be seen:

- The rate of detection of nuclear X-ray sources correlates strongly with host luminosity: the rate of detection is zero below $M_{B} \sim-17$, rises to $\sim 55 \%$ for $-17<M_{B}<-19$, and finally reaches $\sim 100 \%$ for $M_{B}<-19$.

- The distribution of detected sources suggests an upper limit for the nuclear X-ray luminosity that a galaxy can harbour, and that this limit is a linear function of the host luminosity.

- Extended components are fairly common in objects with large X-ray luminosities and large host sizes. 


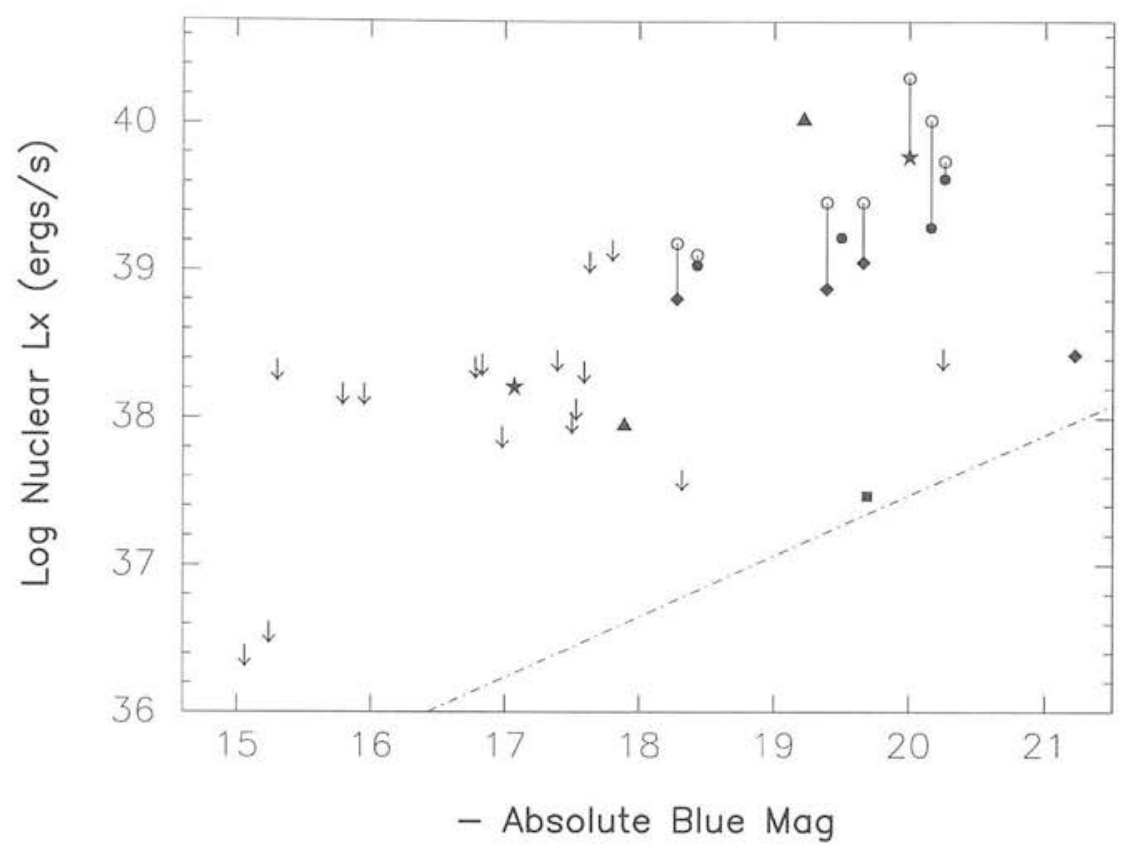

Figure 5.6: Nuclear X-ray luminosity as a function of the host blue absolute magnitude.

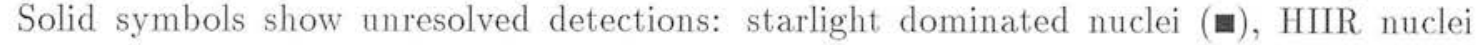
$(\bullet)$, transition objects $(\bullet)$, LINERs $(\star)$, and Seyfert nuclei $(\boldsymbol{\Delta})$. Empty circles show the addition of X-ray flux from an extended component. Arrows correspond to $2 \sigma$ upper limits. The dash-dotted line corresponds to the estimated X-ray luminosity from a population of nuclear LMXRBs (see the star formation model).

- No strong correlation is seen between the optical (spectral) classification of the galaxy and its X-ray luminosity. However, both LINER objects (NGC 404 and NGC 4258) lie close to the upper envelope of the distribution of detected sources, as does the Seyfert galaxy NGC 3031. The second Seyfert nucleus, NGC 4395, is unusually X-ray quiet, as discussed in chapter 4 .

Why does the nuclear X-ray emission (or its upper envelope) correlate with the parent galaxy luminosity? One hypothesis is that the luminosity function of the X-ray source population scales with the galaxy brightness, so that the probability of detecting a source of a particular strength increases with host galaxy luminosity. It is reasonable to assume that galaxies of different luminosities will have scaled versions of a 'universal' luminosity function and the chances of finding a luminous source (or several less luminous sources located in the same region), corresponding to the bright tail of the luminosity distribution, will correlate with the brightness of the host galaxy.

What is this universal luminosity function? M 31 is the only galaxy where the luminosity distribution has been determined to a reasonable degree. Primini, Forman \& Jones (1993) found the cumulative luminosity distribution of X-ray sources within the central $5^{\prime}$. The 


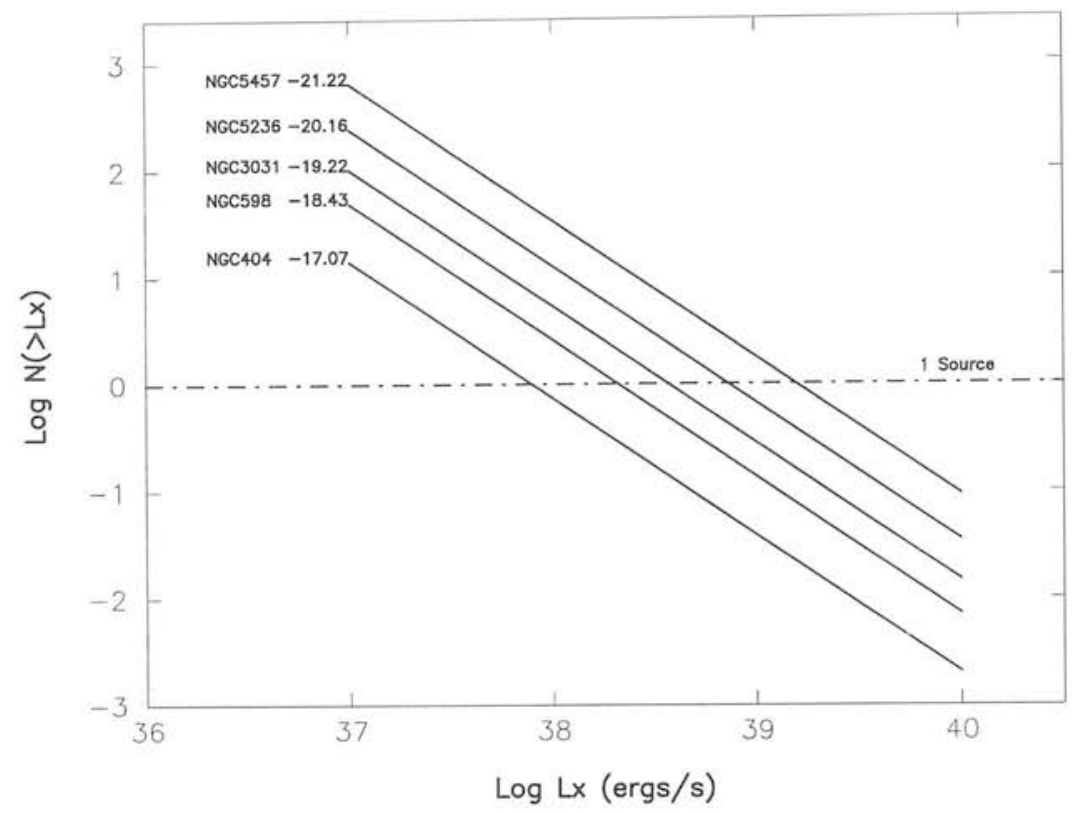

Figure 5.7: Predicted X-ray cumulative luminosity functions for galaxies of different sizes. Each curve has been scaled using the galaxy absolute magnitude (see text).

most luminous source has $L_{X} \gtrsim 10^{38} \mathrm{ergs} \mathrm{s}^{-1}$ and the luminosity dependent distribution shows a clear flattening for sources below $10^{37} \mathrm{ergs} \mathrm{s}^{-1}$. For the following parametrisation

$$
N\left(>L_{X}\right)=K \times\left(\frac{L_{X}}{10^{36}}\right)^{-\alpha}
$$

Primini, Forman \& Jones (1993) found $\alpha=1.74 \pm 0.46$ for sources above $2.0 \times 10^{37}$ ergs $\mathrm{s}^{-1}$ to a significance limit $\sim 14 \sigma$.

Figure 5.7 shows the predicted X-ray luminosity functions for galaxies spanning the whole range of optical luminosities seen in figure 5.6. The distributions have been found using equation 5.2 and by assuming that $K$ scales with galaxy size, i.e., $K=K_{M 31} \times$ ( $L_{g a l} / L_{M 31}$ ), where $K_{M 31}$ is the normalization of equation 5.2 found for $\mathrm{M} 31$, and $L_{g a l}$ and $L_{M 31}$ are the optical luminosities of the galaxies. The dash-dotted line in figure 5.7 shows when the distribution predicts that a source can be detected. Notice that the index $\alpha$ in equation 5.2 has been chosen to be the lowest value allowed by the fit (i.e., $\alpha=1.28$ ) in order to obtain the largest possible X-ray luminosity for the first detected source. As can be seen from figure 5.7 the most powerful sources for galaxies with $M_{B} \sim-20$ magnitudes are predicted to have $L_{X} \sim 10^{39} \mathrm{ergs} \mathrm{s}^{-1}$, while the observations show that galaxies of this brightness can harbour nuclear X-ray sources one order of magnitude brighter. More importantly, the model predicts that a change in the luminosity of the galaxy by $\sim 3$ 


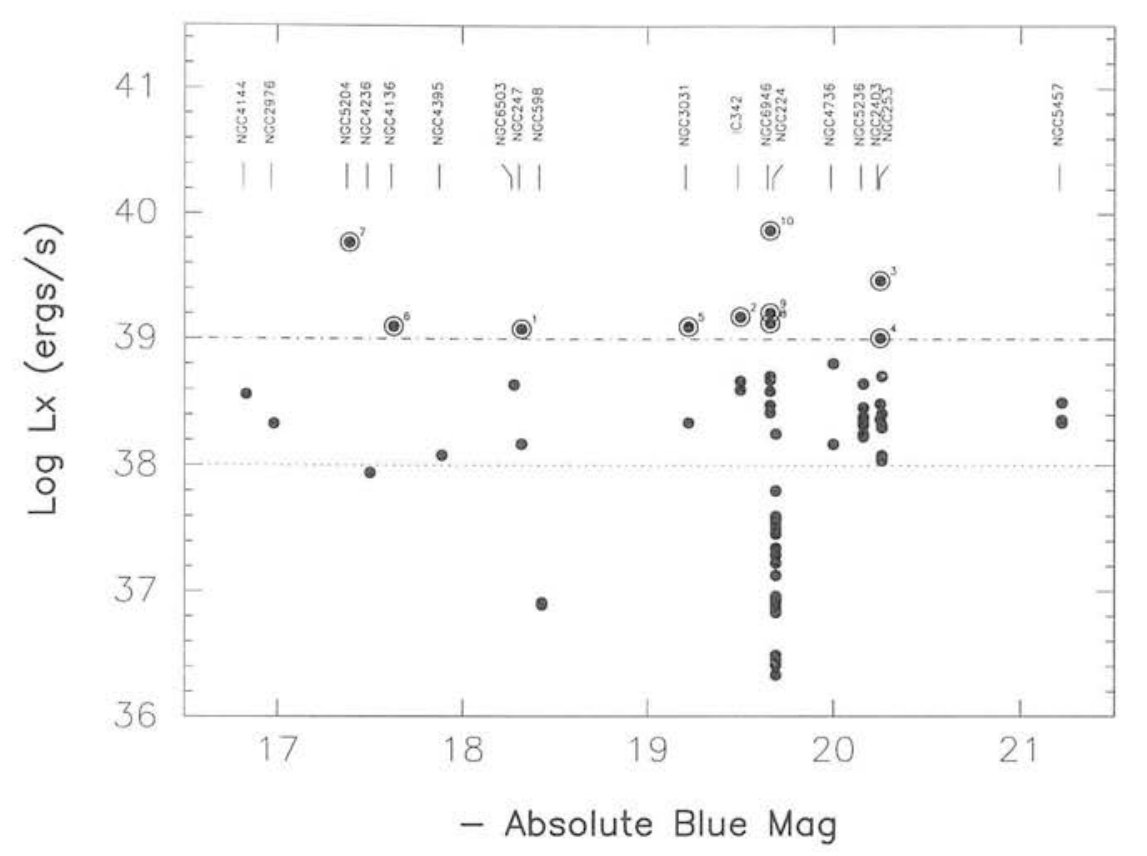

Figure 5.8: X-ray luminosity of off-nuclear sources as a function of the host absolute blue magnitude. The dotted line across the figure shows an estimation of the average sensitivity limit for the HRI observations. Sources with luminosities above $10^{39} \mathrm{ergs} \mathrm{s}^{-1}$ (dash-dotted line) have been labeled. Comments on their identifications can be found in table 5.5.

magnitudes corresponds to a change in the X-ray luminosity by a factor $\sim 10$. Figure 5.6 shows, however, that the $\mathrm{X}$-ray luminosity changes by a factor $\sim 30$. This disagreement is probably even larger, since the flattest possible value for $\alpha$ has been adopted. In other words, the model predicts a too shallow upper envelope, in clear disagreement with the observations.

It is possible that the luminosity function determined by Primini, Forman \& Jones (1993) is not valid for $L_{X}$ above $10^{38} \mathrm{ergs} \mathrm{s}^{-1}$ and that a steeper slope is required. In this case the disagreement between the luminosities predicted for the most powerful sources and the observed values would be even larger.

\subsubsection{Super-luminous X-ray off-nuclear sources}

The argument given in the previous section is not restricted to the nuclear regions, since large galaxies also have more populated disks. Therefore, in principle, the distribution of X-ray luminosities for off-nuclear source should look similar to the one seen in figure 5.6. However, when this figure is reproduced using all off-nuclear sources reported in chapter 2 (except for those cases where the link between the source and the galaxy was not clear, as noted in table 2.4), a clearly different pattern is found: figure 5.8 shows no correlation 


\begin{tabular}{|lllcl|}
\hline \hline Number & Host & ID & $\begin{array}{c}\log L_{X} \\
\left(\mathrm{ergs} \mathrm{s}^{-1}\right)\end{array}$ & Remarks \\
\hline 1 & NGC 247 & $\mathrm{X}-1$ & 39.08 & No optical counterpart \\
2 & $\mathrm{IC} 342$ & $\mathrm{X}-3$ & 39.18 & Possible faint counterpart \\
3 & NGC 2403 & $\mathrm{X}-1$ & 39.47 & No optical counterpart \\
4 & NGC 2403 & $\mathrm{X}-3$ & 39.01 & Possible very faint counterpart \\
5 & NGC 3031 & $\mathrm{X}-6$ & 39.10 & Located on a spiral arm and coincident \\
& & & & with a radio source (Fabbiano 1988a) \\
6 & NGC 4136 & $\mathrm{X}-1$ & 39.10 & Diffuse blue counterpart \\
7 & NGC 5204 & $\mathrm{X}-1$ & 39.77 & Faint counterpart of unknown nature \\
8 & NGC 6946 & $\mathrm{X}-1$ & 39.13 & No optical counterpart \\
9 & NGC 6946 & $\mathrm{X}-7$ & 39.21 & No optical counterpart \\
10 & NGC 6946 & $\mathrm{X}-8$ & 39.87 & Known SNR; faint red counterpart \\
\hline \hline
\end{tabular}

Table 5.5: Luminous off-nuclear X-ray sources. The numbers in the first column correspond to the labels shown in figure 5.8.

between the luminosity of the off-nuclear sources and the brightness of the host galaxy. Remarkably luminous point sources $\left(L_{X}>10^{38} \mathrm{ergs} \mathrm{s}^{-1}\right)$ are seen in galaxies spanning 2 orders of magnitude in luminosity. The most luminous sources $\left(L_{X}>10^{39} \mathrm{ergs} \mathrm{s}^{-1}\right)$ have been labelled with numbers and brief comments about them can be found in table 5.5.

As discussed in chapter 1, the population of X-ray sources in the Milky Way and M 31 does not include luminosities above $10^{38} \mathrm{ergs} \mathrm{s}^{-1}$. The presence of luminous $\left(L_{X} \gtrsim 10^{38} \mathrm{ergs}\right.$ $\mathrm{s}^{-1}$ ) sources in the Magellanic Clouds was assumed to be a metallicity effect (Helfand 1984; Van Paradijs \& McClintock 1995). However, as more galaxies were surveyed using the capabilities of the Einstein and ROSAT satellites it became clear that extremely luminous objects ( $L_{X} \gtrsim 10^{39}$ ergs s$^{-1}$ ) were not rare (Fabbiano 1995). At least one super-luminous source has been identified as a multiple object formed by several interacting SNRs in the disk of NGC6946 (Blair, Fesen \& Schlegel 1997). However, most of these superluminous objects are probably individual sources and, therefore, XRBs (see table 5.4). Their luminosities are several times greater than the Eddington limit for a $\sim 1.4 M_{\odot}$ neutron star. These super-Eddington luminosities could be due to anisotropic emission from neutron stars in binary systems with very strong magnetic fields (Van Paradijs \& McClintock 1995) or black hole XRBs with masses a few times the mass of the sun, which would allow for much higher luminosities without reaching the Eddington limit.

It is clear, then, that super-luminous point sources are common. However, unlike nuclear sources, and as discussed above (figure 5.8), there is no correlation between the probability of having a super-luminous off-nuclear source and the brightness of the parent galaxy. 


\subsection{The nature of the nuclear sources}

The very different correlations between X-ray luminosity and host galaxy absolute magnitude seen in figures 5.8 and 5.6 for nuclear and off-nuclear sources imply two different populations. Nuclear sources are not disk or bulge sources located in the nuclear region by chance. Instead, nuclear sources have a particular nuclear nature.

The next step is to try to unveil this nature. What are these sources? Are they connected with stellar processes in the nuclear region of the galaxies? Could they be an expression of nuclear activity that somehow escapes detection at optical wavelengths?

To assess the nature of the nuclear X-ray sources the observed $H \alpha$ spectral luminosities for the nuclear regions will be used in conjunction with the X-ray measurements. Since the flux calibration for the spectra presented in chapter 3 is highly unreliable, the $\mathrm{H} \alpha$ luminosities determined by Ho, Filippenko \& Sargent (1997b) will be used instead. Their spectra were obtained using an effective aperture of $2^{\prime \prime} \times 4^{\prime \prime}$ with a spatial resolution of $0.4^{\prime \prime}-0.8^{\prime \prime}$ per pixel.

Figure 5.9 shows the correlation between nuclear $\mathrm{X}$-ray and $\mathrm{H} \alpha$ luminosities. The data are shown in table 5.6. The conversion of $F(H \alpha)$ to luminosities has been done assuming the distances given in table 1.1. For those objects with $\mathrm{X}$-ray observation in the Einstein $0.2-$ $4.0 \mathrm{keV}$ band-pass (see table 2.6) a correction factor equal to 1.4 has been applied (Koratkar et al. 1995). NGC 6503 and NGC 5457 are plotted assuming a transition object spectral classification. The dwarf galaxy UGC 6456 has also been included in the figure. The $\mathrm{H} \alpha$ luminosity for the brightest HII region observed in this galaxy is $L(H \alpha)=6.8 \times 10^{37}$ ergs $\mathrm{s}^{-1}$ (Tully et al. 1981). Papaderos et al. (1994) point out that, to account for the total $\mathrm{H} \alpha$ emission, this luminosity has to be scaled by a factor 10 since about that number of bright knots of emission are seen in the central star forming region in UGC 6456.

The positions of two objects seem to disagree with the general trend observed in figure 5.9: NGC 598 and NGC 224 are both separate from the main cluster of points in the Xray $/ \mathrm{H} \alpha$ luminosity plot. It has to be remembered that Ho, Filippenko \& Sargent (1995b) apparently acquired the wrong target when observing NGC 598, and so its $\mathrm{H} \alpha$ luminosity has probably been overestimated (see discussion in chapter 3), so the discrepancy is even stronger.

As mentioned in chapter 2 the high luminosity, slightly extended X-ray source detected in the nucleus of NGC 598 (M 33) is one of the most intriguing nuclear sources seen in 


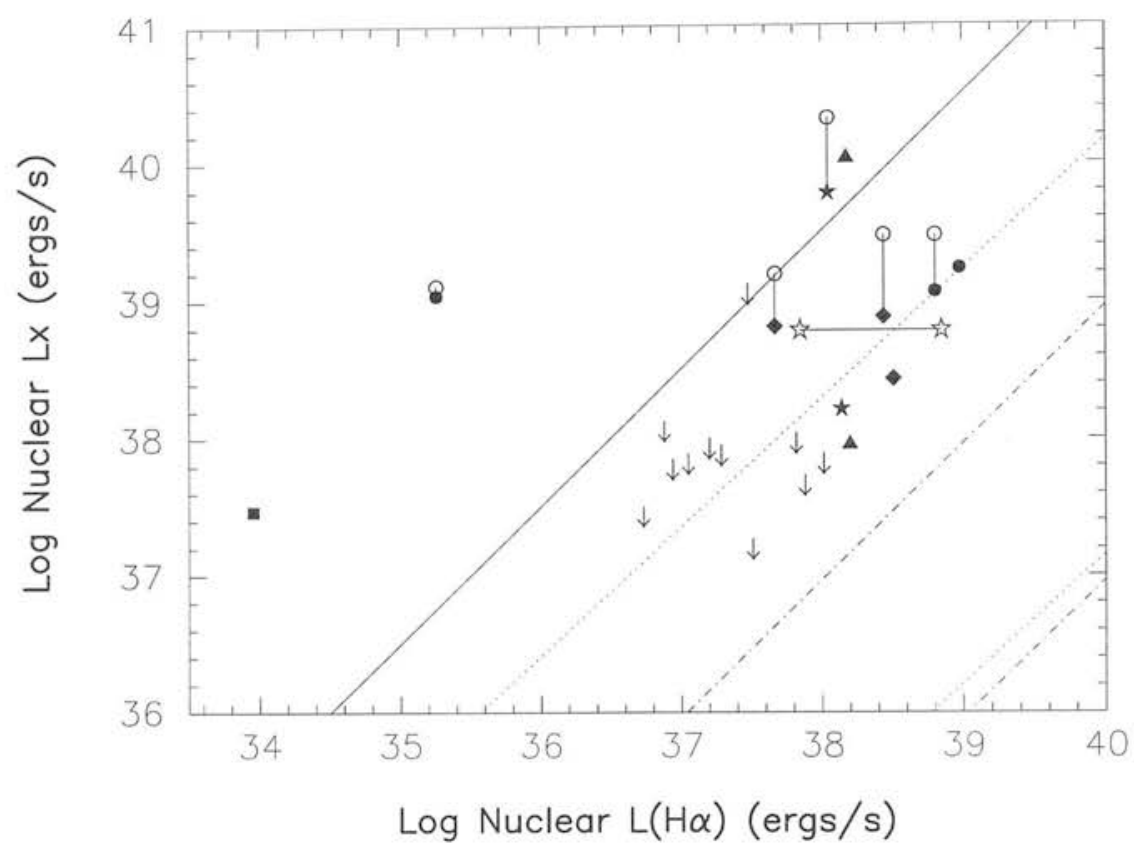

Figure 5.9: Nuclear X-ray luminosity plotted against nuclear $\mathrm{H} \alpha$ luminosity (taken from Ho, Filippenko \& Sargent (1997b)). Solid symbols show unresolved detections: starlight dominated nuclei $(\mathbf{v})$, HIIR nuclei $(\bullet)$, transition objects $(\bullet)$, LINERs $(\star)$, and Seyfert nuclei $(\mathbf{\Lambda})$. Empty circles show the addition of X-ray flux from an extended component. Arrows correspond to $2 \sigma$ upper limits. Observations of the dwarf galaxy UGC 6456 (empty stars) have been included. A correction by a factor 10 in the total $\mathrm{H} \alpha$ luminosity is shown (see text). The solid line shows the correlation between the $\mathrm{X}$-ray and $\mathrm{H} \alpha$ luminosities for AGN (adapted from figure 5 in Koratkar et al. 1995). The dotted and dash-dotted lines are from the star formation model.

nearby galaxies because its soft spectrum is not consistent with the presence of an active nucleus. NGC 224, on the other hand, has a relatively high X-ray luminosity (relative to the $H \alpha$ luminosity). A single XRB coincident with the position of the nucleus is the most obvious explanation for this excess. The observed variability of the source agrees with this scenario (Trinchieri \& Fabbiano 1991).

The observed trend in figure 5.9 can be tested against two hypotheses: first, that the relation is the result of AGN-like activity; and second, that the relation between the Xray and $\mathrm{H} \alpha$ luminosities can be explained as a result of star forming processes in the nuclear region of the galaxies. These two possibilities will be explored in the next two sections.

\subsubsection{The AGN hypothesis}

The X-ray emission from active galactic nuclei correlates with the luminosity seen at other wavelengths. Correlations between X-rays and the continuum at IR, optical and 


\begin{tabular}{|lccc|}
\hline \hline Galaxy & $\begin{array}{c}\log F(\mathrm{H} \alpha) \\
\left(\mathrm{ergs} \mathrm{s}^{-1} \mathrm{~cm}^{-2}\right)\end{array}$ & Class & $\begin{array}{c}\log L_{X} \\
\left.(\mathrm{ergs} \mathrm{s})^{-1}\right)\end{array}$ \\
\hline NGC 404 & -13.21 & $\mathrm{~L} 2$ & 38.21 \\
NGC 598 & -14.70 & $\mathrm{H}$ & $39.04(39.11)$ \\
IC 342 & $-12.67:$ & H & 39.23 \\
NGC 1560 & $-13.47:$ & H & upper limit \\
NGC 2403 & $-14.85:$ & H & upper limit \\
NGC 2976 & -13.70 & H & upper limit \\
NGC 3031 & $-12.73:$ & S1.5 & 39.88 \\
NGC 3738 & -13.63 & H & upper limit \\
NGC 4136 & -13.94 & H & upper limit \\
NGC 4144 & -14.56 & H & upper limit \\
NGC 4150 & -13.87 & T2 & upper limit \\
NGC 4236 & $-14.37:$ & H & upper limit \\
NGC 4244 & -14.41 & H & upper limit \\
NGC 4395 & $-13.31:$ & S1.8 & 37.95 \\
NGC 4605 & -14.10 & H & upper limit \\
NGC 4736 & $-13.60:$ & L2 & $39.78(40.32)$ \\
NGC 4826 & $-13.14:$ & T2 & $38.88(39.47)$ \\
NGC 5204 & $-14.53:$ & H & upper limit \\
NGC 5457 & -13.33 & T2/L2 & 38.43 \\
NGC 6503 & -14.09 & T2/S2 & $38.81(39.19)$ \\
NGC 6946 & $-13.01:$ & H & $39.06(39.47)$ \\
\hline \hline
\end{tabular}

Table 5.6: Nuclear properties of nearby galaxies. Narrow $\mathrm{H} \alpha$ fluxes for objects in the volume-limited sample are taken from Ho, Filippenko \& Sargent (1997b). Observations during non-photometric conditions are indicated with a ' ' ' and are therefore subject to corrections by some unknown factor. The spectral classification is taken from table 5.3. The galaxies are classified as HII nuclei $(H)$, transition objects $(\mathrm{T})$, LINERs $(\mathrm{L})$ and Seyfert nuclei (S). The number attached to the spectral class indicates 'Type-1' or 'Type2' objects, along with intermediate Types (1.2, 1.5, 1.8 and 1.9). In subsequent plots NGC 6503 and NGC 5457 will appear as transition objects. Nuclear X-ray luminosities are taken from table 2.4. Point plus extended luminosities are shown in parentheses. 
UV frequencies have been found in earlier work (Kriss 1988; Mushotzky \& Wandel 1989). Kriss, Canizares \& Ricker (1980) found that Seyfert galaxies show a significant correlation between the $2 \mathrm{keV} \mathrm{X}$-ray flux and the $\mathrm{H} \beta$ line flux.

A large compilation of soft X-ray and broad $\mathrm{H} \alpha$ luminosities by Koratkar et al. (1995) showed a strong correlation between these two quantities, spanning about 6 orders of magnitude. The sample includes low-luminosity AGN with $L(H \alpha) \sim 10^{40} \mathrm{ergs} \mathrm{s}^{-1}$. An indication of the relationship they found $\left(L_{X} \sim L(\mathrm{H} \alpha)+1.5\right)$ is plotted in figure 5.9 with a solid line.

From figure 5.9 it is clear that objects like NGC 3031 and NGC 4736 (both shown using a black triangle) show the expected correlation between $\mathrm{X}$-ray and $\mathrm{H} \alpha$ luminosities. NGC 4395, however, is X-ray underluminous by about 1.5 dex. This confirms the prediction made from the spectral index, $\alpha_{i x}$, in chapter 4 . Notice that the two Seyfert galaxies in figure 5.9, NGC 3031 and NGC 4395, are plotted using their narrow component luminosities and that corrections by factors of $\sim 5$ and $\sim 2$, respectively, are required to obtain the $\mathrm{H} \alpha$ broad component luminosities. Introducing these corrections does not strongly affect the positions of the galaxies in the figure: NGC 3031 is still in very good agreement with the Koratkar correlation and NGC 4395 remains in poor agreement.

The remaining objects in figure 5.9 are all $\mathrm{H} \alpha$ over-luminous compared with the expected AGN correlation. This is in agreement with the idea of an undetected and unresolved AGN $\mathrm{H} \alpha$ component: if an extremely low luminosity active nucleus is present in these galaxies its point-like emission would be buried in the nuclear starlight. From the Koratkar correlation the AGN $\mathrm{H} \alpha$ component, if it exists, should be about ten times fainter than the observed (HII region dominated) emission. In other words, the X-ray nuclear sources could be AGN-like but with their expected $\mathrm{H} \alpha$ components completely masked by the HII emission.

\section{Distribution upper envelopes}

The distribution of X-ray sources with galaxy absolute magnitude, shown in figure 5.6, can be bounded by a nearly linear upper envelope.

A similar linear upper envelope has been found in the correlation between nuclear (AGN) luminosity and host galaxy luminosity for samples of Seyfert galaxies and low redshift quasars at IR and optical wavelengths (Yee 1992; McLeod 1997). This suggests that there is a maximum allowed AGN luminosity which is an increasing function of the luminosity 


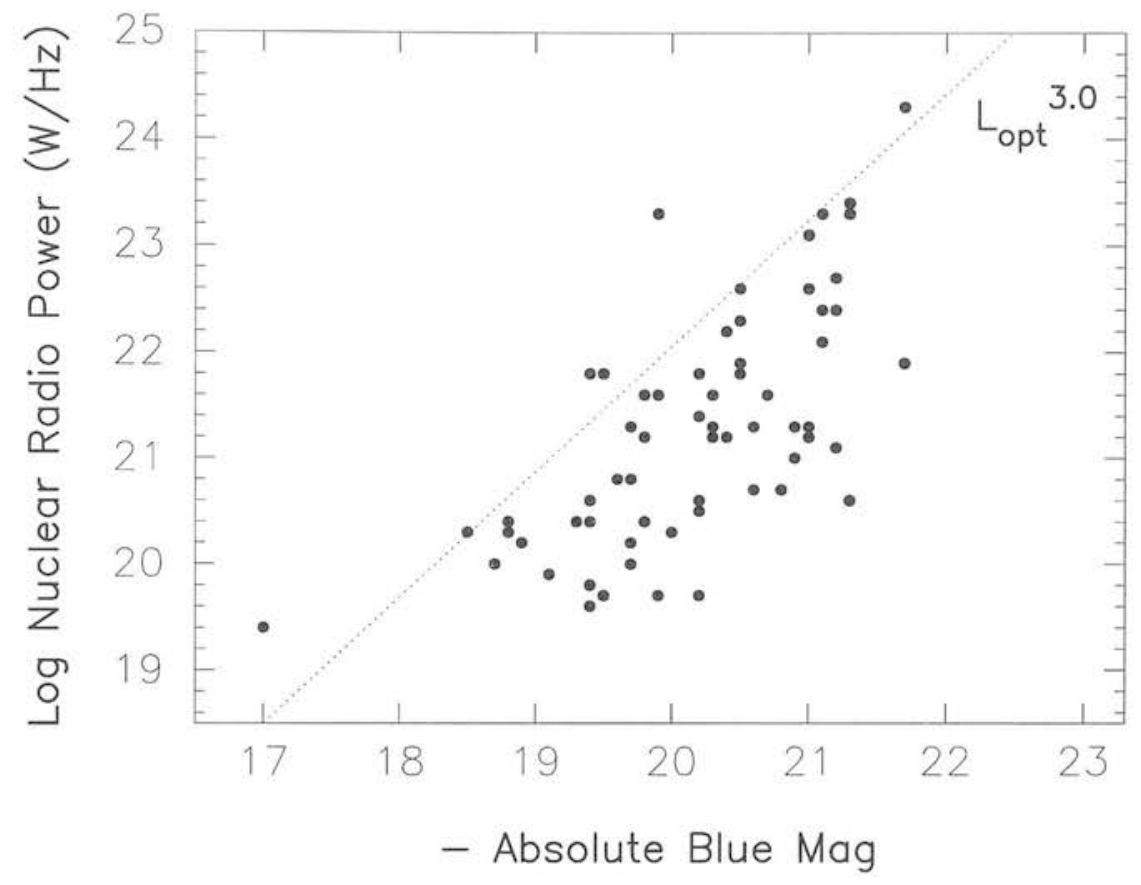

Figure 5.10: Nuclear radio power as a function of host galaxy absolute magnitude (adapted from Sadler, Jenkins \& Kotanyi (1989)).

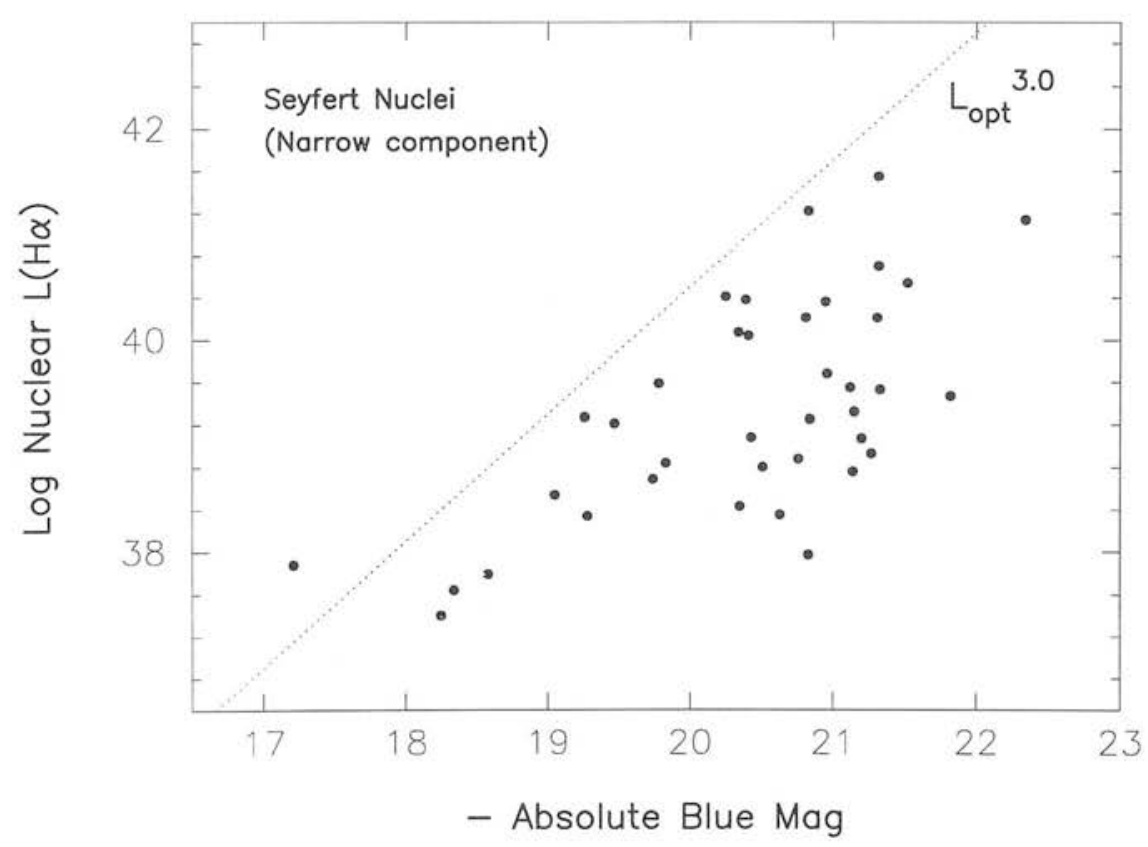

Figure 5.11: Nuclear narrow $\mathrm{H} \alpha$ luminosities versus host galaxy absolute magnitude for galaxies with Seyfert nuclei. Data from Ho, Filippenko \& Sargent (1997b). 


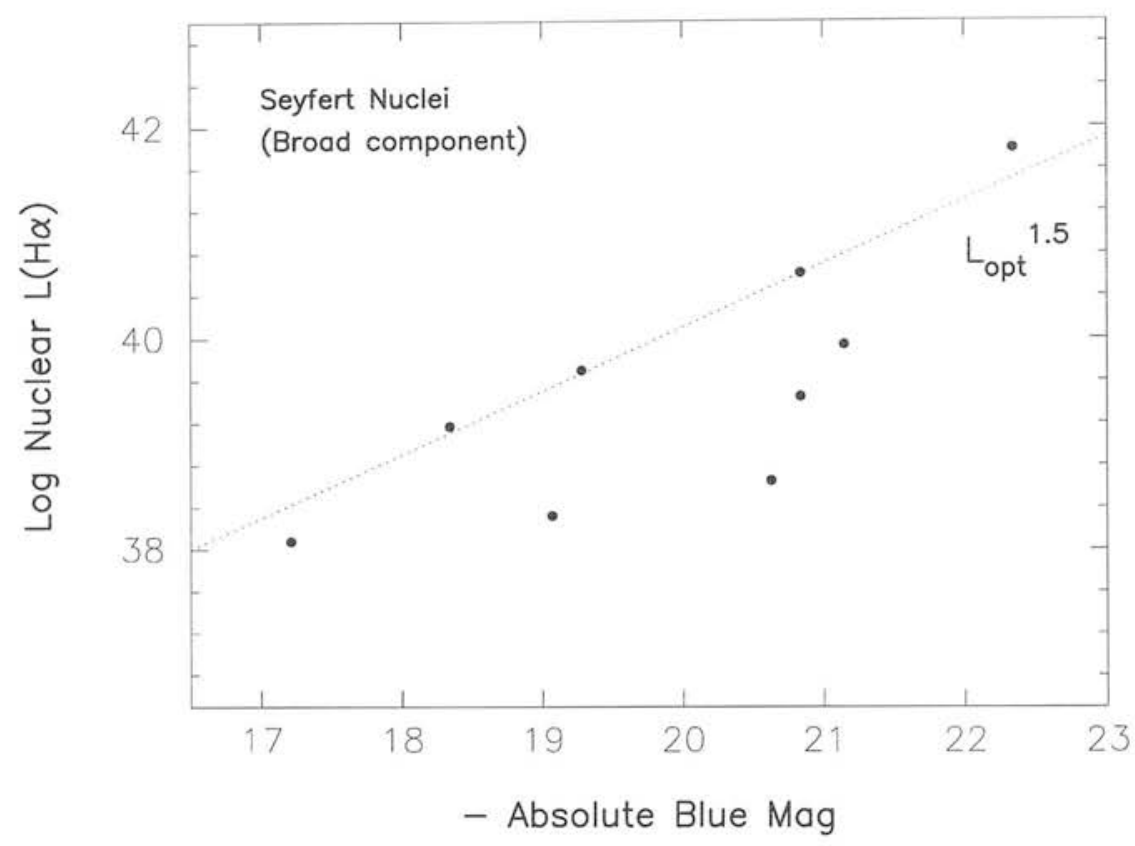

Figure 5.12: Nuclear broad $H \alpha$ luminosities versus host galaxy absolute magnitude for galaxies with Seyfert nuclei. Data from Ho, Filippenko \& Sargent (1997b).

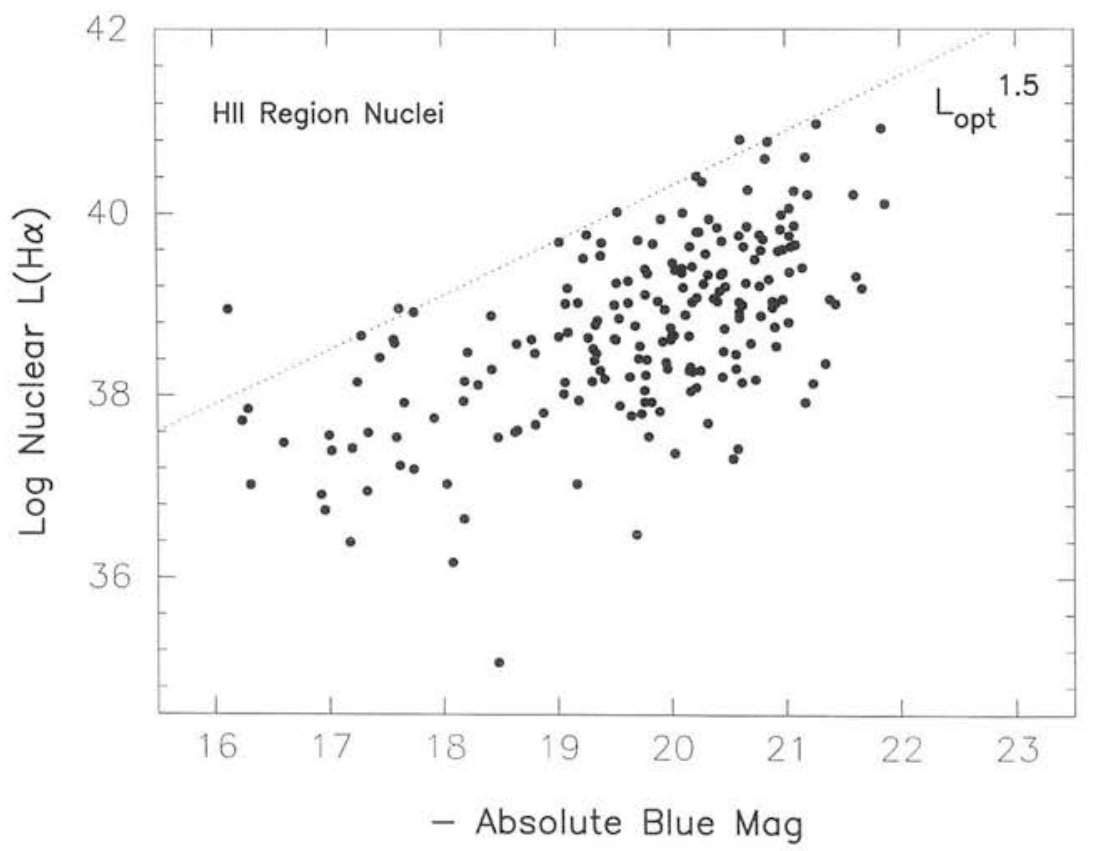

Figure 5.13: Nuclear $\mathrm{H} \alpha$ luminosities versus host galaxy absolute magnitude for galaxies with HII region nuclei. Data from Ho, Filippenko \& Sargent (1997b). 
of the parent galaxy. The limit could be the result of a correlation between the total mass of the host galaxy and the mass of the central engine. The observed range of nuclear luminosities would be given by different accretion rates, with sources accreting at the Eddington limit showing the maximum possible luminosity.

Nuclear radio luminosities seem to depend much more steeply on galaxy luminosity. Sadler, Jenkins \& Kotanyi (1989) searched for weak nuclear radio sources in a sample of 114 nearby E/S0 galaxies and showed that the 'characteristic radio power' (defined as the power above which $30 \%$ of the galaxies have a nuclear source) varies as $L_{\mathrm{opt}}^{2.2}$. Based on their spectroscopic characteristics most of these galaxies have been classified as AGN (Ho 1998). Figure 5.10 reproduces these data to show that the upper envelope to the observations seems even more extreme, with a slope $\sim 3.0$.

It is interesting to compare these results with the dependency between host galaxy absolute magnitude and nuclear $\mathrm{H} \alpha$ luminosity. Figures 5.11, 5.12 and 5.13 show the correlations found for the narrow and broad $\mathrm{H} \alpha$ components in Seyfert galaxies and for HII region nuclei. The data were taken from the Palomar sample (Ho, Filippenko \& Sargent 1997b). A clear difference is seen. While the upper envelope for the distribution of the narrow $\mathrm{H} \alpha$ luminosities in Seyferts is very steep $\left(L_{\mathrm{opt}}^{3.0}\right)$, a much more shallow envelope is observed for the broad component and for HII region nuclei $\left(L_{\mathrm{opt}}^{1.5}\right)$.

Although it is unclear which physical process causes the observed distributions, the upper envelopes give strong evidence for a correlation between the host galaxy luminosity and the level of nuclear activity. The fact that an upper envelope is also observed in the distribution of the nuclear HII regions might suggest that the correlations are governed by the amount of gas available in the nuclear regions. If bigger galaxies are more efficient in dragging gas to their nuclear regions they could show more vigorous star formation and, potentially, feed their massive BHs more efficiently.

What limits the broad $\mathrm{H} \alpha$ component in Seyferts from being more luminous (compared with the steeper correlation seen for the narrow component) is not obvious. The best candidate is that the nuclear sources are reaching their Eddington limits above the observed envelope and, therefore, photoionization is no longer possible. However, this idea is in disagreement with the Eddington ratios inferred from nearby Seyfert galaxies, which appear to be emitting at only a few percent of their Eddington luminosities (Koratkar \& Gaskell 1991). 


\subsubsection{The star formation model}

In this section an attempt will be made to explain the trend observed in figure 5.9 assuming that the $\mathrm{X}$-ray and $\mathrm{H} \alpha$ luminosities are the result of star forming processes in the nuclear region of the galaxies.

High mass XRB and SNR (with Type Ib or Type II progenitors) are the direct end points of the evolution of massive stars. Therefore regions of active star formation show enhanced $\mathrm{X}$-ray luminosities which, in principle, should correlate directly with their $\mathrm{H} \alpha$ luminosities. The X-ray emission from SNRs and XRBs for this scenario will be estimated below.

\section{The sum of individual SNR}

As massive stars have relatively short lifetimes ( $\lesssim 10 \mathrm{Myr}$ ), the X-ray emission from SNRs is proportional to the current star formation rate (SFR), which measures the amount of gas that is converted into stars per unit time. Unfortunately, it is not possible to measure the SFR directly, and indirect techniques have to be used. The most massive stars dominate the Lyman continuum $(\lambda<912 \AA)$ and their presence can be detected as they ionise their surroundings. Therefore, an estimate of the OB SFR can be made by measuring the nebular $\mathrm{H} \alpha$ emission which is, in principle, proportional to the number of Lyman photons (Osterbrock 1989).

An accurate determination of the OB SFR and its extrapolation to a total SFR requires the assumption of a initial mass function (IMF - the mass distribution of newly formed stars) which is not an easy problem to solve for the low-mass end of the distribution. In contrast, studies of globular cluster and nearby starburst galaxies have shown that the upper end of the IMF is well described by a 'universal' function, which is parametrised as a power law with index $\alpha=2.35 \pm 0.5$ (Leitherer 1996), a value first determined by Salpeter (1955), so that, if $d N$ is the SFR per year per mass interval, then

$$
d N=N_{T} M^{-\alpha} d M
$$

where the value of $N_{T}$ sets the total SFR in units of solar masses per year.

Kennicutt (1983) used $\mathrm{H} \alpha$ measurements from a sample of 170 galaxies to obtain quantitative estimates of their SFR. The $\mathrm{H} \alpha$ luminosities were converted to Lyman continuum 


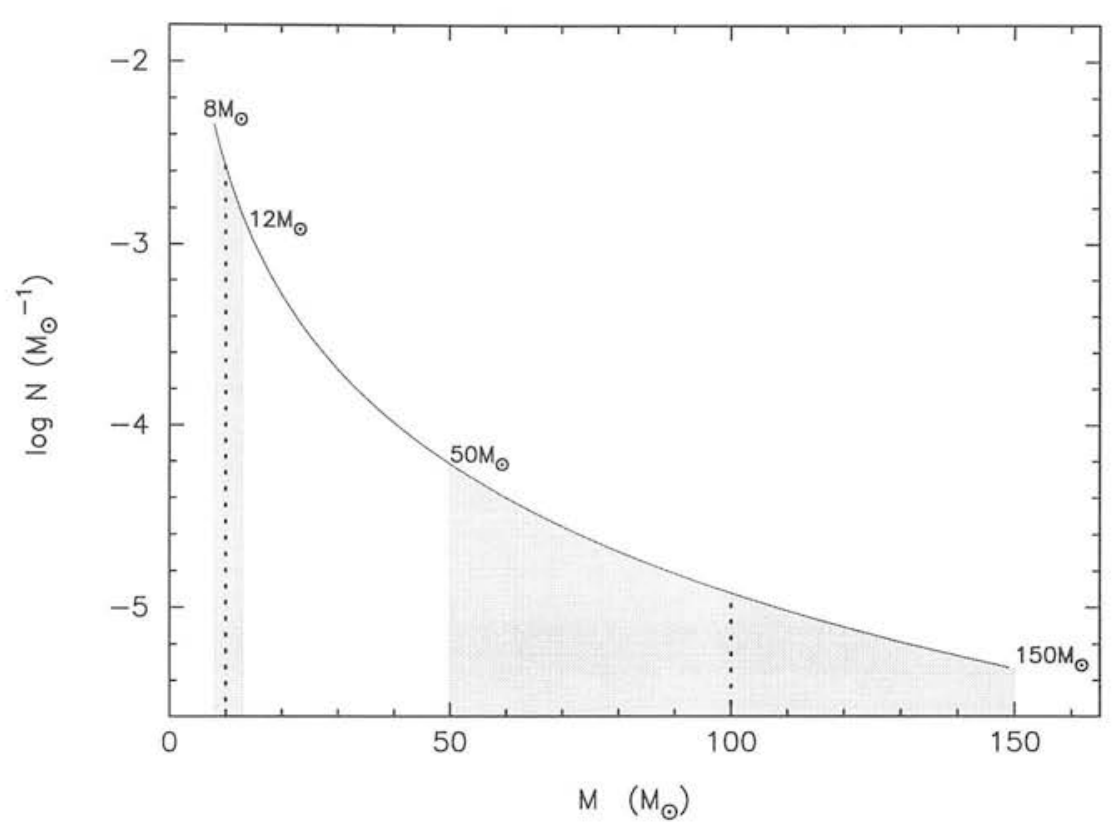

Figure 5.14: Star formation rate between $8 M_{\odot}$ and $150 M_{\odot}$ assuming a Salpeter IMF (see equation 5.3). The curve was normalised assuming a total SFR (between 0.1 and $\left.150 M_{\odot}\right)$ of $10 M_{\odot} \mathrm{yr}^{-1}$. Shaded areas represent masses in the ranges $8-12 M_{\odot}$ and $50-150 M_{\odot}$.

fluxes and combined with evolutionary tracks for massive stars and models of UV emission to yield a relationship between the $\operatorname{SFR}(N)$ and the $\mathrm{H} \alpha$ luminosities:

$$
N\left(\geq 10 M_{\odot}\right)=\frac{L(\mathrm{H} \alpha)}{7.02 \times 10^{41} \mathrm{ergs} \mathrm{s}^{-1}} M_{\odot} \mathrm{yr}^{-1}
$$

The result is mildly sensitive to the (Salpeter) IMF adopted, but more critically, as mentioned before, the extrapolation to a total SFR will be very sensitive to the chosen slope (Kennicutt 1983).

Other methods to trace the current SFR include the measurement of non-thermal radio continuum as an estimate of the synchrotron radiation emitted by relativistic electrons accelerated in SNRs (Condon 1992), and the dust-reprocessed far-IR emission (Hunter et al. 1986; Telesco 1988).

Once the SFR is estimated, the number of SN per unit time will be given by assuming an IMF upper cut-off and a critical mass $\left(M_{c}\right)$ above which all stars produce SN. Figure 5.14 shows the SFR in the $8-150 M_{\odot}$ range of masses, assuming a Salpeter IMF and a total SFR (between 0.1 and $150 M_{\odot}$ ) of $10 M_{\odot} \mathrm{yr}^{-1}$. Shaded areas show the ranges for $M_{c}$ 
and the IMF cut-off normally adopted in the literature (Junkes et al. 1995; Elson, Fall \& Freeman 1989; Kennicutt 1983). The final number of SN is not particularly sensitive to the adopted value of the cut-off (the upper shaded area represents $\sim 15 \%$ of the unshaded area), but it can show variations by a factor of two for the range of $M_{c}$ shown in figure 5.14 (note the logarithmic $y$-axis in the figure).

Using expression 5.4 and assuming a Salpeter IMF, $M_{c}=10 M_{\odot}$, and an upper cut-off for the IMF of $100 M_{\odot}$ (shown as dotted lines in figure 5.14), the number of SN per unit time will given by:

$$
\nu_{S N}=\frac{L(\mathrm{H} \alpha)}{2.19 \times 10^{43} \operatorname{ergs~s}^{-1}} \mathrm{yr}^{-1}
$$

This relationship can now be tested against observations, comparing SN rates from optical surveys with the predicted $\nu_{S N}$ given by equation 5.5. The rate of SN has been taken from the Asiago and Crimea surveys at $0.43 \pm 0.22 \mathrm{SNu}$ (with $1 \mathrm{SNu}=1 \mathrm{SN} \times\left(10^{10} L_{\odot}\right) \times$ $(100 \mathrm{yr})^{-1}$ ) for S0a-Sb galaxies, and $1.75 \pm 0.67 \mathrm{SNu}$ for Sbc-Sd galaxies (Cappellaro 1996). The predicted SN rate is found using equation 5.5 and the total $\mathrm{H} \alpha$ luminosties from the integrated spectra of a sample of nearby galaxies (Kennicutt 1992). The galaxies were binned into the same two morphological groups defined by Cappellaro (1996). Figure 5.15 shows a reasonable agreement between the observations and the model predictions.

Once a relationship between the SN rate and the $\mathrm{H} \alpha$ luminosity has been found it is necessary to estimate the final X-ray emission from the the total population of SNR. The X-ray luminosity of individual SNR can vary substantially, from dim (probably old) remnants with $L_{X} \sim 10^{35} \mathrm{ergs} \mathrm{s}^{-1}$ (Helfand 1984) to the extreme objects found in the Magellanic Clouds with $L_{X} \lesssim 10^{38}$ ergs s$^{-1}$ (Hughes 1987). An estimate of the life time of the X-ray emitting phase is $\tau_{S N R}=2 \times 10^{4} \mathrm{yr}$ (Cioffi 1990; Cowie, McKee \& Ostriker 1981). A relationship between the total X-ray emission from SNRs and the Ho luminosity will then be given by:

$$
L_{X}^{\text {tot }}=L_{X} \times \nu_{S N} \times \tau_{S N R}=9.1 \times 10^{-(2-4)} \times L(\mathrm{H \alpha}) \mathrm{ergs} \mathrm{s}^{-1}
$$

where the range in the exponent reflects the range in SNR X-ray luminosities. Equation 5.6 has been used to infer the limits of the expected X-ray luminosity from the SNR population in a starburst in figure 5.9 (dash-dotted lines). It is clear that the predicted 


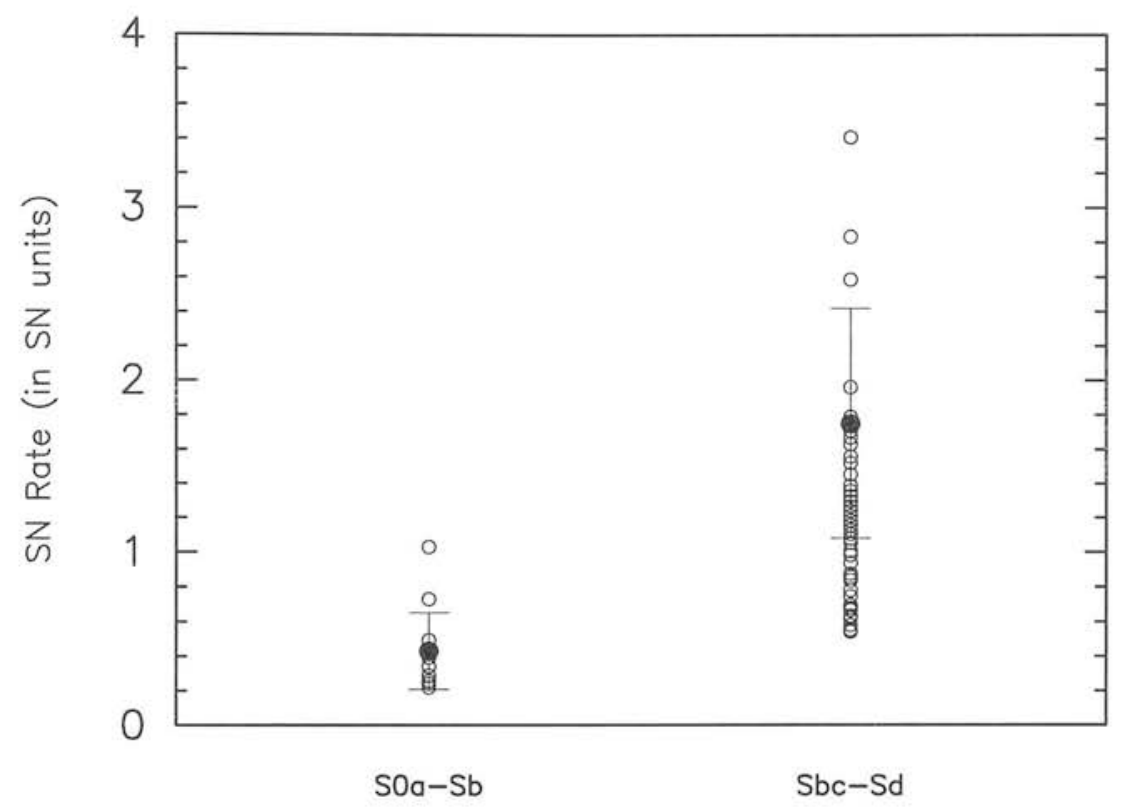

Figure 5.15: Predicted SN rate using equation 5.5 (based on the $\mathrm{H} \alpha$ fluxes from a sample of nearby galaxies (0)) and average measured rates in the Asiago and Crimea surveys as a function of the parent galaxy type $(\bullet)$.

relationship does not agree with the observed range of $\mathrm{X}$-ray and $\mathrm{H} \alpha$ luminosities even in the extremely unlikely situation of all SNR having luminosities as high as $10^{38} \mathrm{ergs} \mathrm{s}^{-1}$. One possible explanation for the disagreement is that the $\mathrm{H} \alpha$ luminosities are severely underestimated, although for most of the objects in figure 5.9 the corrections would need to be of several orders of magnitude which is, again, very unlikely.

\section{The collective effect of SNRs: the bubble scenario}

A more complex scenario than the one described in the previous section arises when the SFR is high enough for SNR to overlap and form a cavity or bubble filled with hot, lowdensity, expanding gas (a 'wind'). When the size of these bubbles becomes comparable to the height scale of the galactic disks, the hot gas will escape into the intergalactic medium.

Observational evidence for the existence of this hot gas comes mainly from the detection of extended X-ray emission well above and below the disk in nearly edge-on nearby starburst galaxies like M 82 and NGC 253 (Watson, Stanger \& Griffiths 1984; Fabbiano \& Trinchieri 1984; Fabbiano 1988b). The detection of this emission is much more difficult to establish for face-on galaxies, where a dim population of point sources in the galactic disk or halo can mimic the distribution of an extended component. 
The theoretical aspects of galactic bubbles and winds have been extensively studied (Heckman, Armus \& Miley 1990; Chevalier \& Clegg 1985; Mac Low \& Mc Cray 1988; Tomisaka \& Bregman 1993) and include models relating the expected X-ray emission to the $\mathrm{H} \alpha$ luminosities. These normally assume spherical symmetry and a continuous and constant deposition of kinetic energy at the centre of the bubble where the starburst is located. The wind expands into the interstellar medium which is assumed to be homogeneous with an exponential density profile. More realistic models, considering a clumpy ambient medium, show that the presence of cool, dense clouds could be an efficient cooling mechanism inside the bubble.

In principle the X-rays will be dominated by the free-free emission from the hot gas whose X-ray luminosity in the ROSAT band is given by (Heckman et al. 1996):

$$
L_{X} \sim 3 \times 10^{40} \dot{E}_{43}^{33 / 35} n_{-2}^{17 / 35} t_{7}^{19 / 35} \mathrm{ergs} \mathrm{s}^{-1},
$$

where $\dot{E}_{43}$ is the rate of energy deposition in units of $10^{43} \mathrm{ergs} \mathrm{s}^{-1}, n_{-2}$ is the ambient medium density in $10^{-2} \mathrm{~cm}^{-3}$ and $t_{7}$ is time in units of $10^{7} \mathrm{yr}$. Two types of object dominate the injection of mechanical energy: stellar winds from massive stars and supernovae. The stellar winds dominate in the very early stages of the starburst (particularly in metal rich clusters), but become negligible once the SN phase has started (Leitherer \& Heckman 1995). Therefore $\dot{E}_{43}$ can be estimated using equation 5.5 and adopting an average energy of $10^{51}$ ergs per $\mathrm{SN}$, giving a relationship between the wind $\mathrm{X}$-ray luminosity and the $\mathrm{H} \alpha$ luminosity, given by:

$$
L_{X} \sim L(H \alpha)^{33 / 35} n_{-2}^{17 / 35} t_{7}^{19 / 35} \text { ergs s}^{-1}
$$

The predictions from this model have been included in figure 5.9 (dotted lines) for two sets of values for the pair $\left(n_{-2}, t_{7}\right):(1,0.1)$ and $\left(10^{4}, 10\right)$. The radiative cooling of the hot gas in the bubble becomes important at $t \gtrsim 10^{8} \mathrm{yr}$ and therefore values for $t_{7}>10$ are not considered.

This time the model predictions are in much better agreement with the observed ranges in $\mathrm{X}$-ray and $\mathrm{H} \alpha$ luminosities for some of the galaxies shown in figure 5.9 - the bubble model gets closer to the observed data than summing individual remnants. For other galaxies, however, important corrections in the $\mathrm{H} \alpha$ luminosities are required before they agree with the upper curve predicted by the model. 
One question that has to be addressed is whether the galaxies in the sample have SFR high enough to drive a wind or not. The model has been developed and tested assuming very high supernova rates which is probably not the case for some of the galaxies seen in figure 5.9. In order to drive a wind the hot gas phase has to dominate the interstellar medium.

This criterion can be expressed in terms of the 'porosity' parameter $P_{H O T}$ (Heckman, Armus \& Miley 1990) given by:

$$
P_{H O T}=1-e^{-Q} \quad \text { with } \quad Q \sim 3 \times S_{-8} n_{0}^{-0.14} P_{7}^{-1.3}
$$

where $S_{-8}$ is the rate of supernova in units of $10^{-8} \mathrm{SN}$ per year per $\mathrm{pc}^{3}, n_{0}$ is the ambient density in units of $100 \mathrm{~cm}^{-3}$, and $P_{7}$ is the ambient pressure expressed as $P / k$ in units of $10^{7} \mathrm{~K} \mathrm{~cm}^{-3}$.

The weak dependency of equation 5.9 on the density implies that, for a wide range of densities, $n_{0}^{-0.14} \sim 1$. The nuclear ambient pressure in normal galaxies has been found to be $z 10^{7} \mathrm{~K} \mathrm{~cm}^{-3}$ (Helfer \& Blitz 1997) and therefore $P_{7}^{-1.3}$ is also of order unity. $S_{-8}$ can be found using equation 5.5 and the typical $\mathrm{H} \alpha$ luminosities seen in figure 5.9. For most galaxies $L(H \alpha)$ is in the $10^{38-39} \mathrm{ergs} \mathrm{s}^{-1}$ range and the emission volume has been estimated at $\sim 3 \times 10^{6} \mathrm{pc}^{3}$ (the volume of a cube with side length $d=\sqrt{8 \operatorname{arcsec}^{2}}-$ the square root of the effective aperture during the acquisition of the spectroscopic data - at a typical distance of $5 \mathrm{Mpc}$ ). This implies $S_{-8} \sim 10^{-(3-4)}$ and therefore $Q \sim 3 \times 10^{-(3-4)}$, corresponding to a porosity $P_{H O T} \ll 1$.

The hot gas dominates the interstellar medium for $S_{-8} \gtrsim 1$ and this requires $L(\mathrm{H} \alpha) \gtrsim 10^{42}$, which is typical for starburst galaxies. Therefore, for the assumed parameter values most of the galaxies in figure 5.9 will not be able to drive a supper-bubble, unless the $\mathrm{H} \alpha$ luminosities have been underestimated by several order of magnitudes.

\section{The possible contribution from XRBs}

As discussed in chapter 1, HMXRB are binary systems containing a young massive star and a compact companion. The compact component, a neutron star or $\mathrm{BH}$, is probably the remnant of the evolution of an even more massive star. To estimate how many HMXRBs are associated with a star forming region it is necessary to establish what fraction of the 
stellar population form such systems. This is an extremely difficult task due to the inherent complexity and many uncertainties in the formation and evolution of binary systems.

Since HMXRB with Be stars are significantly dimmer than binaries with supergiant OB stars (the total luminosity of Be binaries in the Galaxy is estimated to be $\sim 2 \times 10^{36}$ ergs $\mathrm{s}^{-1}$, Van Paradijs \& McClintock 1995), only the population OB binary systems will be taken into account.

Using population synthesis Vanbeveren, De Loore \& Van Rensbergen (1998) have shown that at most $1 \%-2 \%$ of all OB-type stars have a companion neutron star. An even smaller fraction of HMXRBs to OB stars $(\sim 1 / 500)$ has been determined by Fabbiano, Feigelson \& Zamorani (1982). The number of OB stars in a star forming region can be estimated using equation 5.3 for masses above $50 \mathrm{M}_{\odot}$ and assuming an upper cut-off for a Salpeter IMF of $150 M_{\odot}$. Using expression 5.4 to relate the SFR to $H \alpha$ luminosities it is found that the population of $\mathrm{OB}$ stars is given by $\sim 3 \times 10^{-45} \times L(\mathrm{H} \alpha)$. The number of HMXRB will be then given by:

$$
N_{X R B} \sim 3 \times 10^{-45} \times L(\mathrm{H} \alpha) \times f_{X R B} \times \tau_{X R B} \sim 3 \times 10^{-43} \times L(\mathrm{H} \alpha)
$$

where $f_{X R B}(=0.01)$ is the fraction of HMXRB to OB stars and $\tau_{X R B}\left(\lesssim 10^{4} \mathrm{yr}\right.$, Srinivasan $1997)$ is the lifetime of the HMXRB. Given the range of $\mathrm{H} \alpha$ luminosities for the galaxies seen in figure $5.9\left(L(\mathrm{H} \alpha) \sim 10^{(38-39)} \mathrm{ergs} \mathrm{s}^{-1}\right)$, the probability of finding a HMXRB in the nuclear region of the galaxies is found to be very small.

LMXRBs are binary systems that consist of a compact object accreting from a low-mass companion that fills its Roche lobe and tidally loses mass. The typical age of a LMXRB is $\sim 10^{9}$ years and so they are not connected with the current SFR.

The distribution of the Galactic population of LMXRB is well parametrised by an axisymmetric function $\rho(r, z)=\exp \left(-r / r_{0}\right) \exp \left(-z / z_{0}\right)$ with $r_{0} \sim 4.5 \mathrm{kpc}$ and $z_{0} \sim 0.5$ kpc (Van Paradijs \& White 1995). The normalization for this function is given by the observed number of Galactic LMXRB which is thought to be $\sim 100-200$ objects (Van Paradijs 1995). It follows that the number of LMXRB within the central $100 \mathrm{pc}$ of the Milky Way is 2 to 4 , implying an X-ray luminosity of a few times $10^{37} \mathrm{ergs} \mathrm{s}^{-1}$ for an average luminosity per LMXRB of $10^{37} \mathrm{ergs} \mathrm{s}^{-1}$.

Since the LMXRB population does not correlate with the current SFR (and therefore with 
the $H \alpha$ luminosities), but with the past star formation history of the galaxy, it is difficult to estimate the contribution to the nuclear X-ray emission from LMXRB in other galaxies. Assuming that the number of LMXRB scales with host galaxy brightness and using the estimated number of systems in the nuclear region of the Milky Way, the expected number of LMXRB will be $\sim 0.2-0.4$ for a galaxy like NGC 404 with $M_{B} \sim-17$ magnitudes and $\sim 8-16$ for a galaxy like NGC 5457 with $M_{B} \sim-21$ magnitudes. The luminosity associated with these sources is shown in figure 5.6 as a dash-dotted line.

There is a large uncertainty in these extrapolations. This is shown by M31, which has a brightness similar to the Milky Way. The spatial distribution of bulge sources (spectral observations of M 31 with Ginga (2-20 keV) suggest that the overall X-ray emission in this galaxy is dominated by LMXRBs - Makishima et al. 1989), however, is much more concentrated towards the nucleus than in the Milky Way, with an enhancement by a factor 4 in the central $5^{\prime}$ (Primini, Forman \& Jones 1993). Naively, therefore, the expected Xray luminosity is $\sim 10^{38}$. However, the observed nuclear X-ray source has a luminosity $\sim 2 \times 10^{37}$ ergs s$^{-1}$.

From figure 5.6 it is clear that although a population of LMXRBs could explain part of the $\mathrm{X}$-ray nuclear luminosities the observed X-rays are normally 1 to 2 orders of magnitude more luminous (except for the cases of M 31 and possibly NGC 5457). It should be noted that the calculations done here are very uncertain and a better understanding of the past star formation history of each individual galaxy is required to assess the LMXRB population more accurately.

\section{Corrections to the $\mathrm{H} \alpha$ luminosities}

The results from the previous sections show that if the observed X-ray nuclear emission is a result of star formation activity then the implied $\mathrm{H} \alpha$ luminosities are greater than those inferred from observations. There are two possible explanations for this problem: either the galaxies have severe internal extinction, or the measured $\mathrm{H} \alpha$ emission has been underestimated. These possibilities will be discussed below.

\section{Extinction corrections}

It is well known that in starburst galaxies the visual extinction towards the nucleus can be as large as a few tens of magnitudes (see for example McLeod et al. 1993 for extinction estimations in the starburst galaxy M 82). However, for the galaxies seen in figure 5.9, 


\begin{tabular}{|lccrr|}
\hline \hline Galaxy & $E(B-V)^{\text {Int }}$ & $E(B-V)^{\text {Gal }}$ & $A_{V}^{\text {Tot }}$ & $\Delta \log L(H \alpha)$ \\
\hline NGC 404 & 0.13 & 0.06 & 0.59 & 0.19 \\
NGC 598 & 0.41 & 0.04 & 1.40 & 0.46 \\
IC 342 & 0.14 & 0.56 & 2.17 & 0.71 \\
NGC 3031 & 0.00 & 0.08 & 0.25 & 0.08 \\
NGC 4395 & 0.00 & 0.02 & 0.06 & 0.02 \\
NGC 4736 & 0.01 & 0.02 & 0.09 & 0.03 \\
NGC 4826 & 0.53 & 0.04 & 1.77 & 0.58 \\
NGC 5457 & 0.33 & 0.01 & 1.05 & 0.34 \\
NGC 6503 & - & 0.03 & $\geq 0.09$ & $\geq 0.03$ \\
NGC 6946 & 1.16 & 0.34 & 4.65 & 1.53 \\
\hline \hline
\end{tabular}

Table 5.7: Internal extinction estimates for objects in the volume-limited sample with detected X-ray nuclear sources (from Ho, Filippenko \& Sargent (1997b)). $A_{V}^{\text {Tot }}=3.1 \times$ $\left(E(B-V)^{\mathrm{Int}}+E(B-V)^{\mathrm{Gal}}\right)$ (in magnitudes).

there is no evidence to support this.

Table 5.7 gives the internal extinction for those galaxies with X-ray nuclear detections shown in figure 5.9 assuming an intrinsic $\mathrm{H} \alpha / \mathrm{H} \beta$ ratio of 2.86 (Ho, Filippenko \& Sargent 1997b). The Galactic extinction values are taken from Schlegel, Finkbeiner \& Davis (1998). The values of $A_{V}^{\text {Tot }}$ show that some of the galaxies might have up to 5 magnitudes of total visual extinction. However, the corresponding extinction corrections to the logarithm of the $\mathrm{H} \alpha$ luminosities $\left(\Delta \log L(H \alpha)=A_{\lambda}^{\text {Tot }} / 2.5\right.$, where $\lambda=6563 \AA$ and the extinction law determined by Cardelli, Clayton \& Mathis (1989a) was used) are still quite small, except for NGC 6946 and IC 342, and are not able to explain the deficit in $\mathrm{H} \alpha$ emission found earlier.

\section{$\mathrm{H} \alpha$ extended profiles}

As discussed earlier, the luminosity of a single knot in UGC6456 is only a tenth that of the total inferred $\mathrm{H} \alpha$ emission for the galaxy. Similar corrections for extended or multiple sources may also be needed for the estimated luminosities of the galaxies shown in figure 5.9. The $\mathrm{H} \alpha$ fluxes were obtained by Ho, Filippenko \& Sargent (1995b) using a $2^{\prime \prime} \times 4^{\prime \prime}$ aperture (equivalent to $50 \times 200 \mathrm{pc}^{2}$ for a galaxy at a distance of $5 \mathrm{Mpc}$ ). Since in some of the galaxies there is clear evidence of X-ray emission extending several arc-seconds away from the galactic nucleus it is important to check whether the $\mathrm{H} \alpha$ emission is similarly extended. To see whether these corrections are necessary, the long slit spectroscopic data shown in chapter 3 will be used.

Of the galaxies listed in table 5.6 with detected $\mathrm{X}$-ray emission (i.e., excluding those objects 

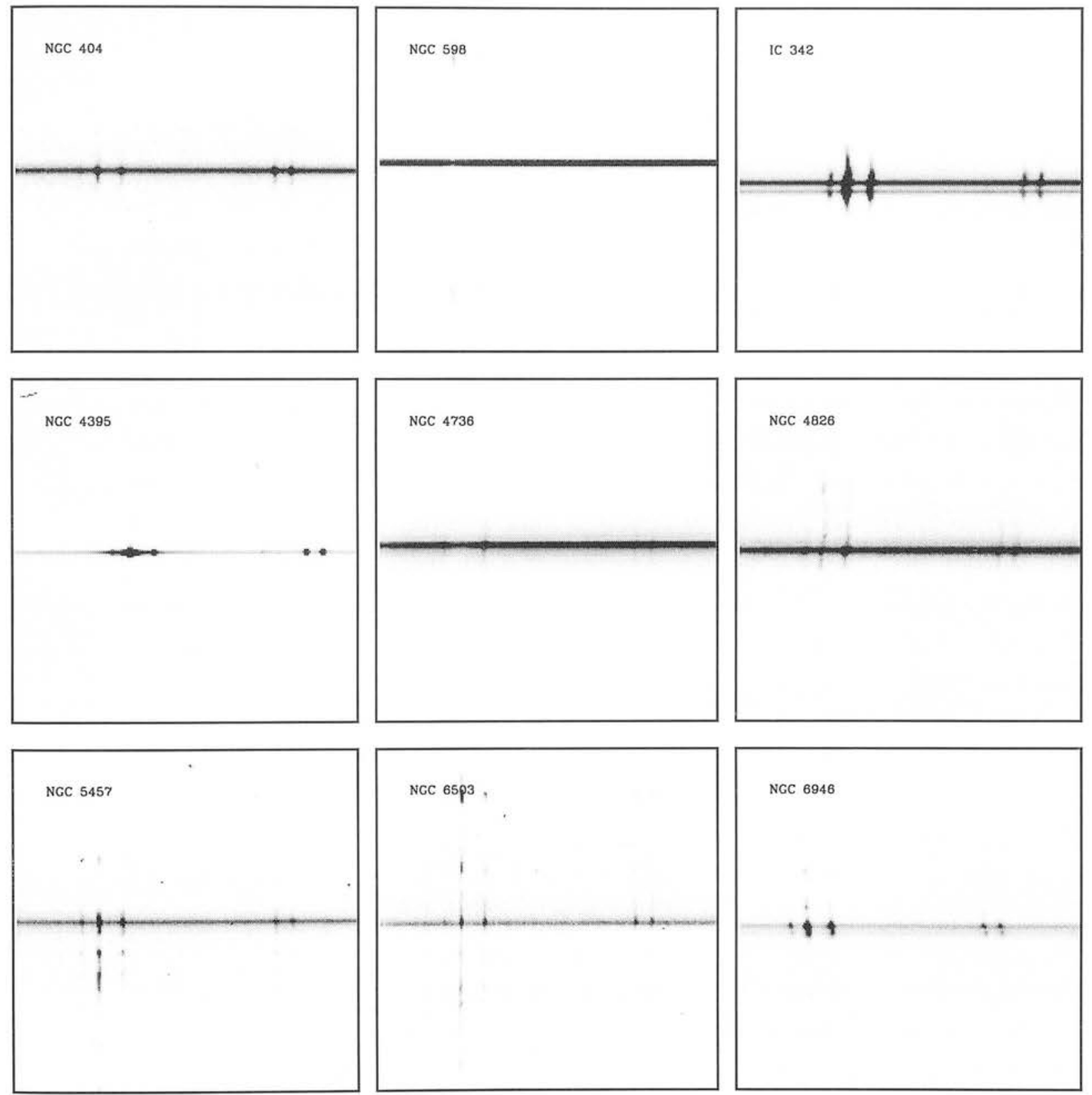

Figure 5.16: Long slit spectroscopic images in the $\sim 6480-6770 \AA$ range for objects that have detected nuclear X-ray emission. 
with only observational upper limits) the nuclear $\mathrm{H} \alpha$ emission of NGC404, NGC 598, NGC 4395 and NGC 4736 appear point-like and do not seem to be significantly extended (see figure 5.16). As discussed earlier, the nuclear $\mathrm{H} \alpha$ luminosity for NGC 598 has probably been overestimated and an inspection at the long slit data shows that no extended $\mathrm{H} \alpha$ emission is observed in the nuclear region. No spectral long-slit observations exist for NGC 3031 .

Figures $5.17,5.18$ and 5.19 show the $\mathrm{H} \alpha$ spatial profiles observed at the parallactic angle for four galaxies with detected X-ray nuclear sources and $\mathrm{H} \alpha$ extended emission: IC 342, NGC 4826, NGC 6503 and NGC 6946. The extended X-ray profiles obtained in chapter 2 have been also included in the figures and scaled to match the central peak of the $\mathrm{H} \alpha$ emission (gray thick lines), as have the model PSFs fitted to the HRI observations (thin dotted lines).

The observed $\mathrm{H} \alpha$ morphologies are complex and in general do not show good agreement with the azimuthally averaged X-ray profiles, particularly for NGC5457 and NGC6503 where the $\mathrm{H} \alpha$ emission is significantly more extended than in the $\mathrm{X}$-ray data. It should be remembered, however, that the spectral data have been obtained at a single position angle through the nucleus of the galaxies and that 2 dimensional $H \alpha$ observations are required for a thorough comparison of the morphologies. For an idea of the noise in the $\mathrm{X}$-ray features, figures $5.17,5.18$ and 5.19 should be compared with the profiles shown in chapter 2 .

For IC 342 and NGC 4826 the central emission profiles at both wavelengths have similar widths. For NGC 6946 the X-ray emission departs from the model PSF only for a radius $z 3^{\prime \prime}$ - no obvious extended $\mathrm{H} \alpha$ emission is seen along the slit at this radius. NGC 5457 and NGC 6503 show complex and extended $\mathrm{H} \alpha$ emission in disagreement with the X-ray profiles which show narrower profiles.

The next step is to determine the corrections to apply to the H $\alpha$ luminosities measured by Ho, Filippenko \& Sargent (1997b), which were measured through a $2^{\prime \prime} \times 4^{\prime \prime}$ aperture, in order to find the total emission. Gaussian profiles were fitted to the peaks seen in figures 5.17 and 5.18. The integral of a 2D Gaussian generated as solid rotation of the 1D profile is given by

$$
I=2 \pi A \sigma^{2}
$$


IC342

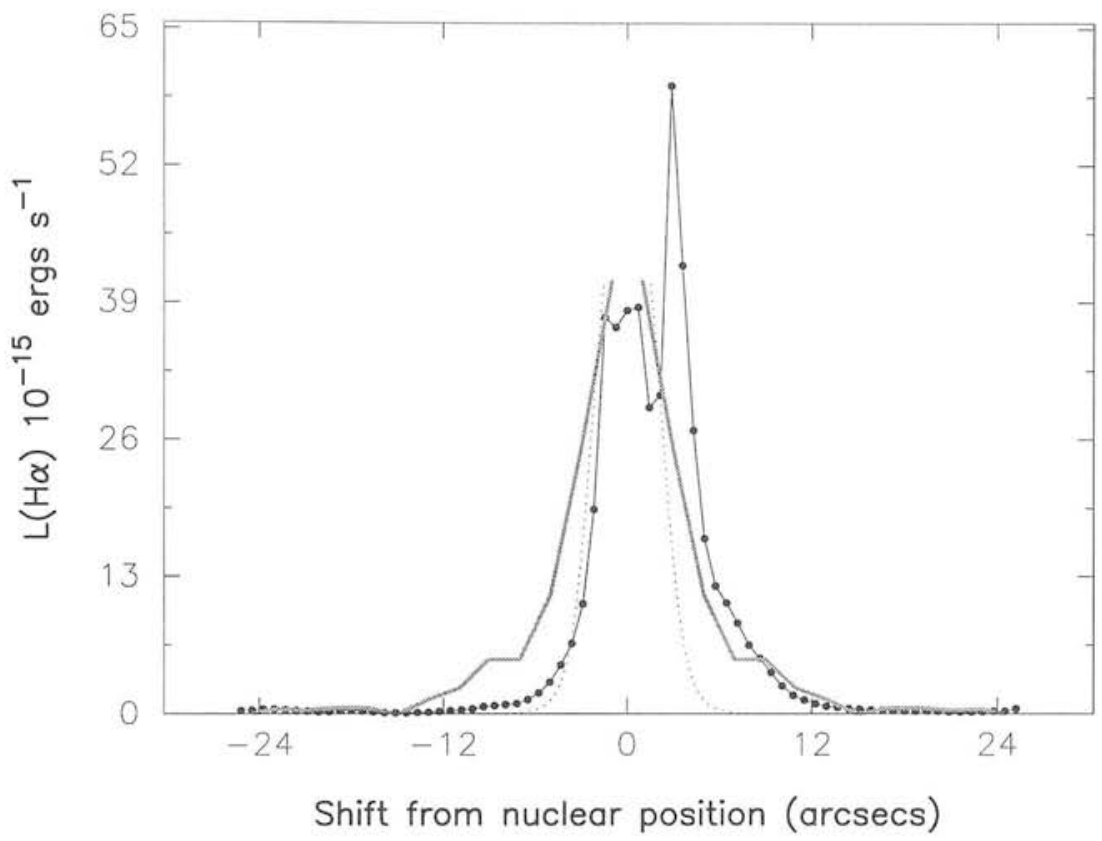

NGC4826

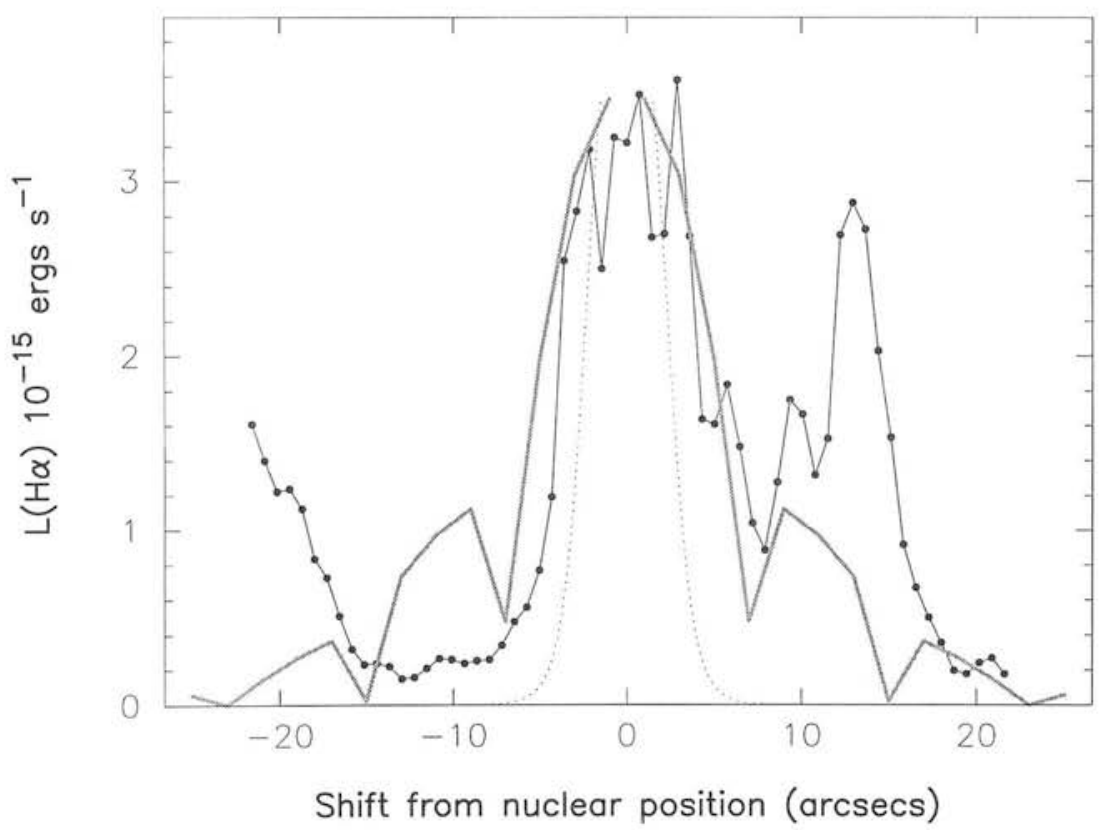

Figure 5.17: Observed $H \alpha$ (lines with black dots) and X-ray (thick gray lines) extended profiles. For comparison, the model PSFs for the HRI observations have also been included (thin dotted lines). 
NGC5457

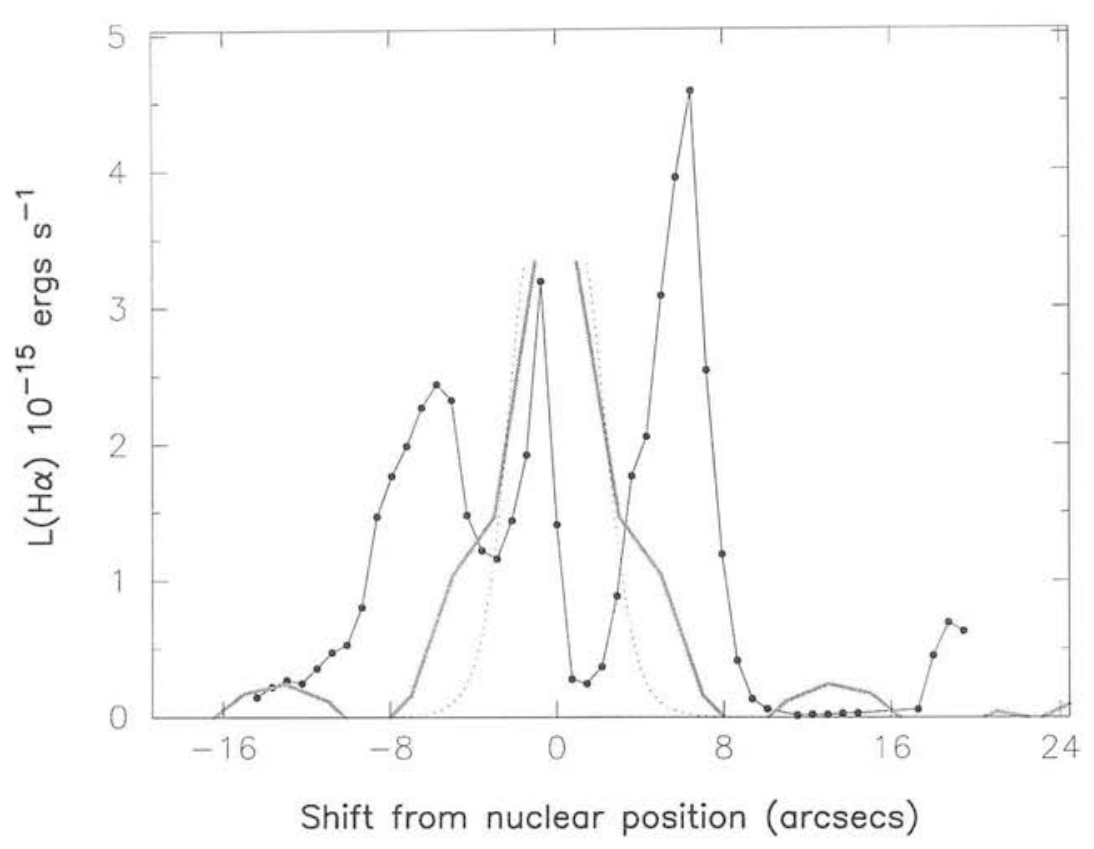

NGC6503

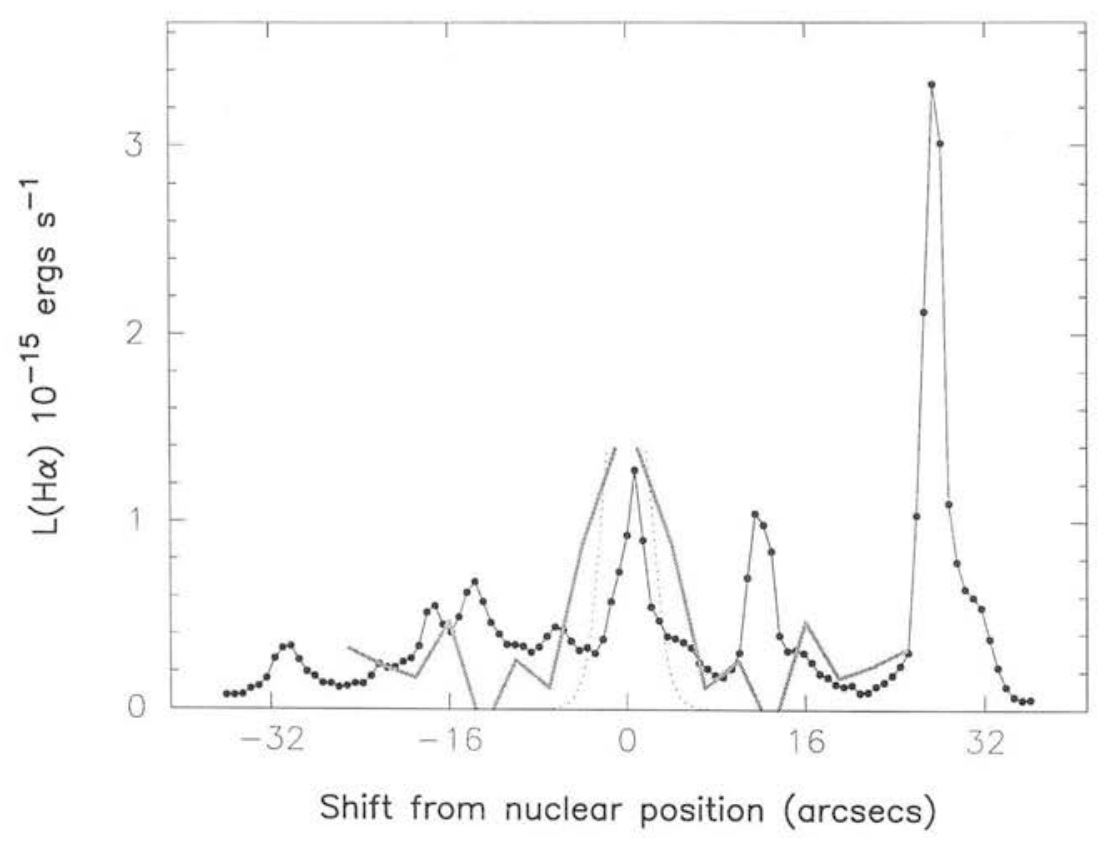

Figure 5.18: Observed $\mathrm{H} \alpha$ (lines with black dots) and X-ray (thick gray lines) extended profiles. For comparison, the model PSFs for the HRI observations have also been included (thin dotted lines). 


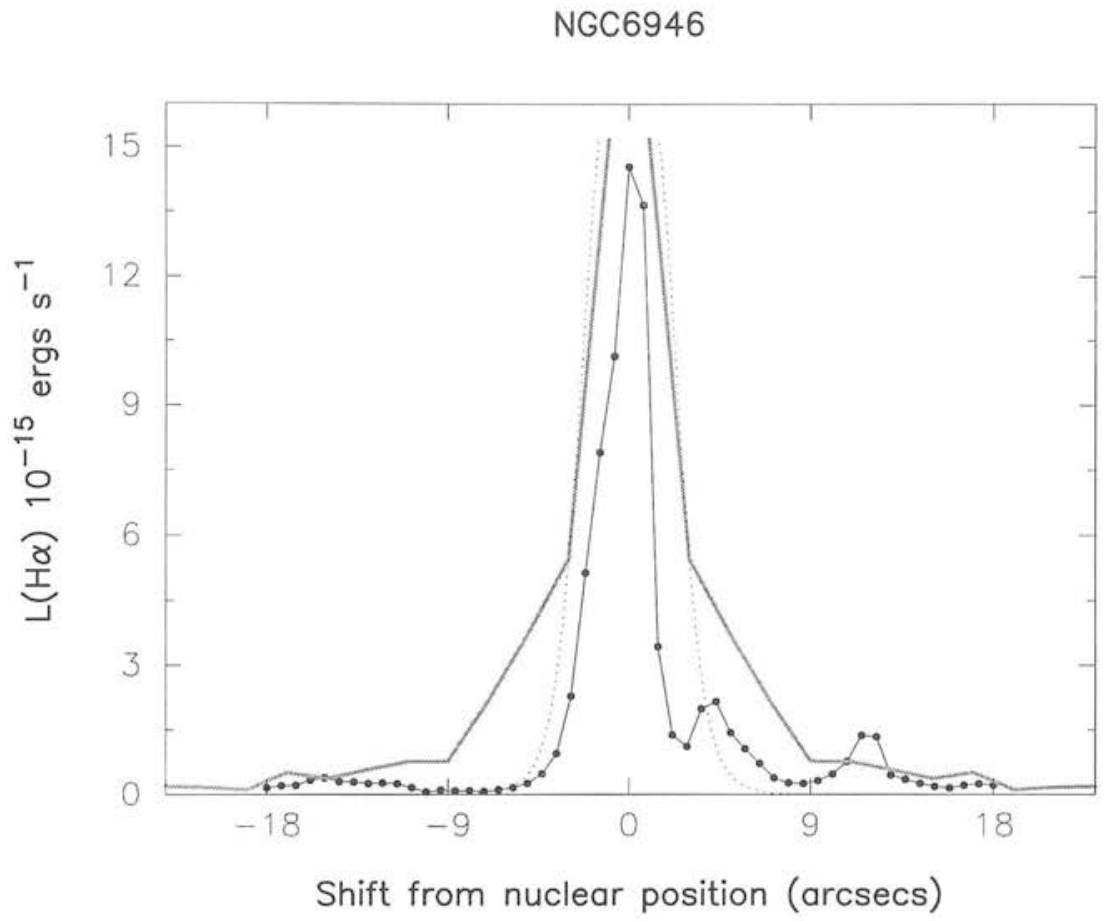

Figure 5.19: Observed $H \alpha$ (lines with black dots) and X-ray (thick gray lines) extended profiles. For comparison, the model PSFs for the HRI observations have also been included (thin dotted lines).

\begin{tabular}{|lccccc|}
\hline \hline Galaxy & $\begin{array}{c}\log A \\
\left(\mathrm{ergs} \mathrm{s}^{-1} \operatorname{arcsec}^{-2}\right)\end{array}$ & $\begin{array}{c}\sigma \\
(\operatorname{arcsec})\end{array}$ & $\begin{array}{c}\log L(\mathrm{H} \alpha)^{\text {Obs }} \\
\left(\mathrm{ergs} \mathrm{s}^{-1}\right)\end{array}$ & $\begin{array}{c}\log L(\mathrm{H} \alpha)^{\text {Tot }} \\
\left(\mathrm{ergs} \mathrm{s}^{-1}\right)\end{array}$ & $\log \left(L^{\text {Tot }} / L^{\text {Obs }}\right)$ \\
\hline IC 342 & 37.93 & 3.05 & 38.98 & 39.70 & 0.72 \\
NGC 4826 & 37.35 & 3.87 & 38.44 & 39.33 & 0.88 \\
NGC 5457 & 37.75 & 1.05 & 38.51 & 38.59 & 0.08 \\
NGC 6503 & 36.70 & 1.75 & 37.67 & 37.99 & 0.32 \\
NGC 6946 & 37.98 & 1.19 & 38.81 & 38.93 & 0.12 \\
\hline \hline
\end{tabular}

Table 5.8: Parameters of the Gaussian fits to the extended $\mathrm{H} \alpha$ profiles and observed and corrected (total) $\mathrm{H} \alpha$ luminosities. 


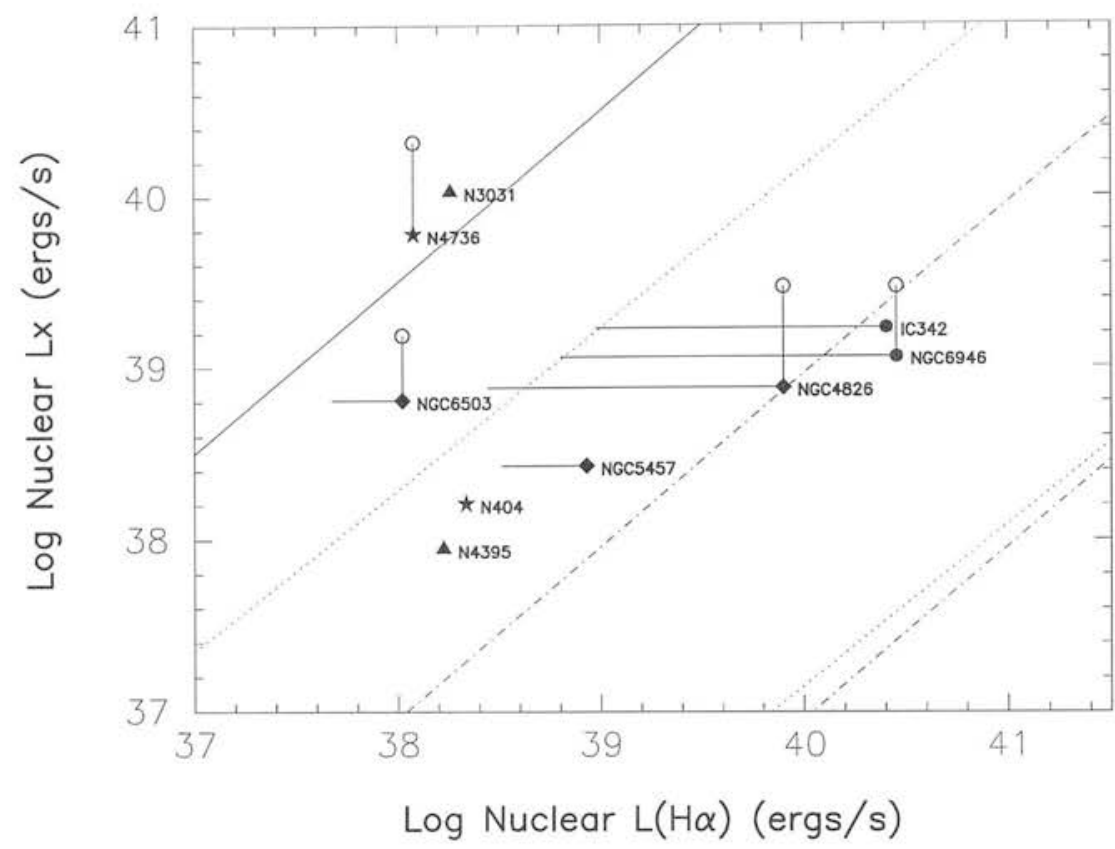

Figure 5.20: Nuclear X-ray luminosity plotted against nuclear $\mathrm{H} \alpha$ luminosity after correcting by the total extinction and estimating the total $\mathrm{H} \alpha$ emission. Symbols are the same as in figure 5.9. The dotted and dash-dotted lines correspond to the estimated X-ray $/ \mathrm{H} \alpha$ correlations for the super-bubble model and for a population of nuclear SNRs, respectively.

where $A$ is the amplitude of the 1D Gaussian and $\sigma$ is its standard deviation. The normalisation of the amplitudes is determined using the $\mathrm{H} \alpha$ luminosities determined by Ho, Filippenko \& Sargent (1997b) and assuming that the apertures (equal to $8 \mathrm{arcsec}^{2}$ ) were centered on the Gaussian distributions of the $\mathrm{H} \alpha$ emission. Table 5.8 shows the fitted Gaussian parameters and the observed and corrected luminosities. The last column in the table shows that for galaxies with broad emission peaks, like IC 342 and NGC 4826, corrections by about one order of magnitude are appropriate.

For NGC 6946 the narrow spatial profile seen in figure 5.18 implies that most of the emission was seen through the slit.

NGC 5457 and NGC 6503 show extended morphology within a radius of $\lesssim 16^{\prime \prime}$ and $\lesssim 30^{\prime \prime}$ from the nuclei respectively, and it is clear than the estimate of the total emission in table 5.8 is not appropriate. In cases like these it becomes difficult to adopt good criteria for what is 'nuclear' and what is not. 


\section{Final $\mathrm{H} \alpha$ luminosities}

Figure 5.20 reproduces the $\mathrm{X}$-ray $/ \mathrm{H} \alpha$ plot taking into account the corrections for extinction and underestimated $H \alpha$ nuclear emission. For those galaxies where the corrections were found to be larger than a factor 2, the shift introduced by the new $\mathrm{H} \alpha$ luminosities are shown. For three objects the corrections are uncertain: NGC3031 does not have an estimation of the total amount of $\mathrm{H} \alpha$ emission, NGC 6503 has only an upper limit to the internal extinction and its total nuclear $\mathrm{H} \alpha$ flux is probably larger than that found in the previous section, as is the case for NGC 5457 .

For three galaxies (IC 342, NGC 4826 and NGC 6946) the corrections have been significant. Their final $\mathrm{H} \alpha$ luminosities should be regarded as good estimates of the true values since satisfactory corrections for the spatial extent of the emission and of the degree of extinction have been applied. These three objects are now much closer to the ranges of $\mathrm{H} \alpha$ and $\mathrm{X}$-ray luminosities predicted by the star formation model. It has to be remembered, however, that galaxies with $L(H \alpha) \sim 10^{40}$ are not expected to efficiently drive a super-bubble and, therefore, the dash-dotted line representing the X-ray emission from a collection of individual SNRs is the appropriate model.

Other interesting comments associated with figures 5.9 and 5.20 are: (1) the presence of extended bright X-ray emission in NGC 4736, NGC 4826, and NGC 6946 is difficult to explain outside the super-bubble model; (2) the locations of NGC 3031 and NGC 4736 in figure 5.20 are in clear disagreement with the star formation model; and (3) given the observed $\mathrm{H} \alpha$ luminosities most objects in figure 5.20 seem to be X-ray over-luminous with respect to the star formation model predictions.

\subsubsection{Conclusions}

In trying to unveil the nature of the X-ray sources in the nuclear regions of galaxies two possible scenarios have been presented: that the nuclear emission is driven by an active nucleus, or that it is produced by nuclear star formation.

Given that the physics of AGN are not well understood, the AGN hypothesis has been analysed in a rather empirical manner. The observations have been compared with correlations observed for more luminous AGN, extrapolating them to the luminosity ranges observed in the volume-limited sample.

A better understanding of star formation processes allows the star formation hypothesis 
to be tested more thoroughly - the conclusions reached under this scenario are on much firmer grounds.

Neither of the hypotheses explains all the data. A few objects are in excellent agreement with the correlations seen for brighter AGN. However, most of the galaxies have $\mathrm{H} \alpha$ luminosities that exceed what is predicted from their X-ray luminosities. On the other hand, following the star formation model, most of the objects do not have sufficient $\mathrm{H} \alpha$ luminosity to explain the X-ray emission, even under the most optimistic assumptions.

Therefore, in order to explain both the observed X-ray and $\mathrm{H} \alpha$ luminosities, the two models must be combined with active nuclei dominating X-ray emission and star formation activity dominating at optical wavelengths. X-ray spectra and variability information from the isolated (X-ray) nuclear source are required to test this. 


\section{Chapter 6}

\section{Summary}

This thesis has presented X-ray images and optical spectra that were obtained for a volumelimited sample of galaxies. Chapters 2 and 3 described how the data were acquired and reduced, and also reported on the major results.

Chapter 4 presented conclusive evidence for $\mathrm{X}$-ray and optical variability in the active nucleus of one galaxy in the sample - the nearby spiral galaxy NGC 4395. This object had been classified, from its optical emission line spectrum, as a Seyfert galaxy. However, being an extremely faint nucleus located in a small galaxy, the nature of the observed emission had been questioned and star formation activity had been proposed as an alternative explanation of the observations. In particular, the lack of detected variability was a major obstacle to the AGN hypothesis. Optical and X-ray data presented here show clear evidence of variability which not only mimics the patterns observed amongst more luminous AGN, but also suggests that NGC 4395 could vary in a much more extreme way than its brighter relatives.

The results from the first chapters were used to gain a deeper insight into the role of nuclear activity amongst small galaxies. The main questions addressed were: (1) whether AGN are restricted to big, luminous galaxies, or if they are also present in small galaxies; and (2) if the correlation between the level of nuclear activity and the luminosity of the parent galaxy seen at higher luminosities is still present in the fainter objects.

The optical spectroscopic classification for the nuclei of the galaxies in the volume-limited sample was given in chapter 5 . The relative numbers of active and normal nuclei were found to agree with the results from a major spectroscopic survey carried out by Ho, Filippenko \& Sargent (1995a; 1997a; 1997c; 1997d). Analysis using both surveys together showed that the fraction of active galaxies declines steadily as a function of host absolute magnitude 
from $\sim 0.8$ for $M_{B} \sim-24$ to $\sim 0.1$ for $M_{B} \sim-17$. For dimmer objects marginal evidence suggested that the distribution does not drop to zero for $M_{B} \gtrsim-17$, but remains constant at a very small value.

Analysis of the X-ray emission from the galaxies in the volume-limited sample, also presented in chapter 5 , showed some surprising results. Both the probability of harbouring an X-ray nuclear source and the luminosity of that source correlated with the absolute magnitude of the host galaxy. A completely different picture was found for off-nuclear sources, where super-luminous sources $\left(L_{X} \gtrsim 10^{38-39} \mathrm{ergs} \mathrm{s}^{-1}\right)$ did not show any correlation with the luminosity of the parent galaxy. Finally, an extended component of the nuclear X-ray emission was common amongst the most luminous galaxies.

The physical nature of the nuclear sources was investigated. The observed $\mathrm{X}$-ray and $\mathrm{H} \alpha$ luminosities were compared with the empirical correlation found for more luminous AGN, and with models of star formation activity. Although a few objects could be explained by either the AGN or the star formation hypothesis alone, most galaxies would require a combination of both models. In these cases the AGN would dominate the nuclear X-ray emission while the star formation activity would provide the emission line spectra seen at optical wavelengths. 


\section{Appendix A}

\section{X-ray emitting mechanisms.}

The four most common processes responsible for X-ray emission are: black body radiation, Bremsstrahlung (or free-free) emission, synchrotron radiation and inverse Compton scattering. While black body and (thermal) free-free radiation are described as thermal processes, synchrotron radiation and Compton scattering are non thermal, in the sense that the energy distribution of the particles involved cannot be described in terms of a single temperature $\mathrm{T}$.

\section{A.1 Black Body Radiation}

In a medium with optical depth $\tau \rightarrow \infty$, the radiation will be in thermal equilibrium and its intensity will depend solely on the temperature $T$.

The spectrum of black body radiation is given by the Planck function:

$$
I(T)=\frac{2 h \nu^{3} / c^{2}}{e^{h \nu / k T}-1}
$$

(in units of energy per unit time, unit volume, unit frequency and unit solid angle). The radiation of a black body with temperature $z 10^{6}$ degrees will peak at soft X-ray frequencies. The most likely sources of this emission are the neutron stars newly formed during a supernova explosion. 


\section{A.2 Thermal Free-Free or Bremsstrahlung Emission}

In a hot, optically thin gas, free electrons will be accelerated under the presence of ions of charge $Z e$ and will radiate. Assuming a thermal distribution of the electron velocities it is possible to show that the intensity of the output radiation is given by:

$$
I(E, T)=6.8 \times 10^{-38} G(E, T) Z^{2} n_{i} n_{e} T^{-1 / 2} e^{-E / k T} \quad\left(\operatorname{ergs~s}^{-1} \mathrm{~cm}^{-3} \mathrm{~Hz}^{-1}\right)
$$

where $E=h \nu$ is the photon energy, $T$ is the gas temperature, $n_{e}$ and $n_{i}$ are the electron and ion densities, and $G(E, T)$ is the 'Gaunt correction factor' to the classical treatment to account for quantum effects during the collision processes. No radiation is produced during encounters of similar particles (e.g. electron-electron) since there is no net dipole associated with the collision.

The cut off in the above expression shows that in a Maxwellian distribution of velocities and for high energies $(h \nu \gg k T)$ the number of electrons capable of producing photons of frequency $\nu$ will decay exponentially.

A low density hot gas (plasma) will also contain elements partially ionized, such as oxygen, carbon, etc, depending on the gas temperature and composition. Collisions between free electrons and these ions will produce emission lines in the output spectrum. If the temperature is too high, almost all the atoms will have lost their electrons and the lines will be weaker. At $10^{7} \mathrm{~K}$ half of the X-ray emission is radiated in lines and half as free-free emission. At $\mathrm{T} \gtrsim 10^{8} \mathrm{~K}$ almost all atoms will have lost their electrons and the spectrum will show very few emission lines (e.g., see Sarazin \& Bahcall 1977).

\section{A.3 Synchrotron radiation}

Relativistic charged particles in the presence of a magnetic field $B$ will radiate due to the acceleration exerted by the magnetic forces. The motion of the particles will consist of a constant velocity along the magnetic field $\left(v_{\|}\right)$and a circular motion on the plane perpendicular to $B\left(v_{\perp}\right)$. The centripetal acceleration due to the change in the direction of $v_{\perp}$ is given by: 


$$
a_{\perp}=v_{\perp}^{2} / r=Z e v B \sin \theta / \gamma m_{0} c
$$

where $r$ is the gyroradius of the particle, $\gamma=\left(1+(v / c)^{2}\right)^{-1 / 2}$ is the particle Lorentz factor, $\theta$ is the angle between $B$ and the velocity $v$, and $m_{0}$ and $Z e$ are the rest mass and charge of the particle. The angular frequency or gyrofrequency of the rotation is given by:

$$
\omega=2 \pi \nu=v_{\perp} / r=Z e B / \gamma_{0} m_{0} c .
$$

The total radiated power from an isotropic distribution of velocities (i.e. averaging over the pitch angle $\theta$ ) is:

$$
P=\frac{4}{9} r^{2} c\left(\frac{v}{c}\right)^{2} \gamma^{2} B^{2}=\frac{4}{3} \sigma_{T} c\left(\frac{v}{c}\right)^{2} \gamma^{2} U_{B}^{2},
$$

where $\sigma_{T}=8 \pi r^{2} / 3$ is the Thompson cross section, and $U_{B}=B^{2} / 8 \pi$ is the magnetic energy density.

The cycling path of a single electron in the magnetic field direction will beam the radiation towards the observer, resulting in a series of pulses shorter than $1 / \omega$ by a factor $\gamma^{3}$, and therefore a spread over wavelength range much broader than $2 \pi / \omega$.

The overlapping of pulses from an isotropic population of electrons with a power law kinetic energy distribution with index $p$ (i.e., $n(\gamma) \propto \gamma^{-p}$ ) will produce an output spectrum with a power law distribution of index $-(p-1) / 2 \sim-1$, which will dominate emission at low frequencies.

At sufficiently low frequencies self absorption of the synchrotron radiation becomes important. A self absorbed spectrum of a source that has becomes optically thick to synchrotron radiation will have a power law index of $5 / 2$ at frequencies lower than the transitional frequency $\nu_{m}$.

\section{A.4 Inverse Compton Scattering}

Inverse Compton scattering involves the scattering of low energy photons to high energies by ultrarelativistic electrons. The process results in the increase of the photon energy by 
a factor $\gamma^{2}$, where $\gamma$ is the electron Lorentz factor. The maximum attainable energy $\epsilon$ is given by $\epsilon^{\prime}<\gamma m_{0} c^{2}+\epsilon$, where $\epsilon^{\prime}$ is the photon energy before the collision.

At high energies the effectiveness of the process is reduced due to the introduction of quantum corrections to the Thompson cross section (given by the Klein-Nishina formula), which reduces the efficiency of the scattering.

The total power emitted by an electron under the presence of an isotropic radiation field is given by:

$$
P=\frac{4}{3} \sigma_{T} c\left(\frac{v}{c}\right)^{2} \gamma^{2} U_{\text {rad }}^{2}
$$

where $U_{\text {rad }}$ is the energy density of the radiation field, provided that, in the rest frame of the electron, $h \nu^{\prime} \ll m_{0} c^{2}$ (with $h \nu^{\prime}=$ the energy of the incident photon), so that the interaction is well described by Thompson scattering.

To obtain the emergent spectrum it is necessary to know both the spectrum of the incident radiation field and the energy distribution of the relativistic electrons. In an analogous way to Synchrotron radiation, the Comptonisation of photons with a power-law distribution of electron energies will produce scattered radiation with the spectrum $I(\nu) \propto \nu^{-(p-1) / 2}$.

Ultrarelativistic electrons with, for example, $\gamma=1000$ will produce a final photon $10^{6}$ times more energetic than the incoming photon. This Compton scattering process will shift photons in the far-IR wavelengths $\left(z 10^{12} \mathrm{~Hz}\right)$ to X-ray frequencies $\left(z 10^{18} \mathrm{~Hz}\right.$, or $\sim 10 \mathrm{keV})$. 


\section{Appendix B}

\section{Statistical formulism}

\section{B.1 Confidence limits for Poisson Statistics}

When working with small numbers of counts $(N)$ drawn from a Poisson distribution the approximation of a $1 \sigma$ (Gaussian) confidence level as $\sqrt{N}$ becomes inaccurate. The problem arises not just in the estimate for $\sigma$, but also in the fact that the shape of the distribution becomes increasingly asymmetric.

Gehrels (1986) has shown that if $N$ events drawn from a Poisson distribution are detected, then the upper $\left(\lambda_{u}\right)$ and lower $\left(\lambda_{l}\right)$ limits at a confidence level CL are given by:

$$
\begin{aligned}
& \sum_{x=0}^{N} \frac{\lambda_{u}^{x} e^{-\lambda_{u}}}{x !}=1-\mathrm{CL} \\
& \sum_{x=0}^{N-1} \frac{\lambda_{l}^{x} e^{-\lambda_{l}}}{x !}=\mathrm{CL} \quad N \neq 0
\end{aligned}
$$

Unfortunately it is not possible to analytically solve equations B.1 and B.2 for $\lambda_{u}$ and $\lambda_{l}$. Using numerical techniques Gehrels (1986) tabulated values of $\lambda_{u}$ and $\lambda_{l}$ for $N=0$ to $N=50$ and for different (and most commonly used in Astronomy) values of CL. He has also deduced approximate algebraic expressions for both limits which are accurate to within $1 \%$ for $N<10$ at a $1 \sigma(0.8413)$ confidence level: 


$$
\begin{gathered}
\lambda_{u} \approx(N+1)\left(1-\frac{1}{9(N+1)}+\frac{S}{3 \sqrt{N+1}}\right)^{3} \\
\lambda_{l} \approx N\left(1-\frac{1}{9 N}-\frac{S}{3 \sqrt{N}}\right)^{3} \quad N \neq 0
\end{gathered}
$$

where $S$ is the equivalent number of Gaussian $\sigma$ s corresponding to the confidence level.

\section{B.2 The Kolmogorov-Smirnov test}

The Kolmogorov-Smirnov (KS) test compares the cumulative distribution of a one-dimensional data-set with a model, or compares the cumulative distribution of two different data-sets or samples. In the latter case no previous knowledge of the parent population is required. One of the most important characteristics of the KS test is that no binning of the data is necessary and therefore all points in the sample are used. When comparing two datasets the test gives the likelihood that the samples have been drawn from the same parent population.

The KS statistic corresponds to the maximum absolute vertical distance between the two distributions within a specified range of the measured variable:

$$
D=\max _{x}\left|S_{n 1}(x)-S_{n 2}(x)\right|
$$

where $S_{n 1}(x)$ and $S_{n 2}(x)$ are the two samples with $n 1$ and $n 2$ data points respectively. Once the statistic has been determined its significance can be computed using:

$$
Q(\xi)=2 \sum_{j=1}^{\infty}(-1)^{j-1} \exp \left(-2 j^{2} \xi^{2}\right)
$$

where

$$
\xi=D\left(\sqrt{N}+0.12+\frac{0.11}{\sqrt{N}}\right) \quad N=\frac{n 1 n 2}{n 1+n 2}
$$


Press et al. (1992). $Q(\xi)$ is a monotonic function with limit values 1 and 0 . The null hypothesis that the two samples have been drawn from the same parent population can be rejected if $Q(\xi)$ is smaller than an adopted value. A conservative significance level to reject the null hypothesis is found for $Q(\xi) \leq 0.01$, while suggestive evidence for its rejection is found for $0.01<Q(\xi) \leq 0.10$. 


\section{Bibliography}

Aretxaga I., Terlevich R., 1994. Mon. Not. R. Astr. Soc., 269, 462.

Aretxaga I., Cid Fernandes R., Terlevich R. J., 1997. Mon. Not. R. Astr. Soc., 286, 271.

Baldwin J. A., Phillips M. M., Terlevich R. J., 1981. Publ. Astron. Soc. Pacific, 93, 5.

Barbon R., Capellaro E., Turatto M., 1989. Astron. Astrophys. Suppl., 81, 421.

Bartel N., Shapiro I. I., Corey B. E., Marcaide J. M., Rogers A. E. E., Whitney A. R., Cappallo R. J., Graham D. A., Romney J. D., Preston R. A., 1982. Ap. J., 262, 556.

Barth A. J., Reichert G. A., Filippenko A. V., Ho L. C., Shields J. C., Mushotzky R. F., Puchnarewicz E. M., 1996. Astron. J., 112, 1829.

Beck S. C., Beckwith S. V., 1984. Mon. Not. R. Astr. Soc., 207, 671.

Bender R., Kormendy J., Dehnen W., 1996. Ap. J. Lett., 464, L123.

Bica E., Alloin D., Schmidt A. A., 1990. Astron. Astrophys., 228, 23.

Bica E., 1988. Astron. Astrophys., 195, 76.

Blair W. P., Fesen R. A., Schlegel E. M., 1997. American Astronomical Society Meeting, $190,2706$.

Bohlin R. C., Cornett R. H., Hill J. K., Smith A. M., Stecher T. P., 1983. Ap. J. Lett., 274, L53.

Boksenberg A., Netzer H., 1977. Ap. J., 212, 37.

Boller T., Bertoldi F., Dennefeld M., Voges W., 1998. Astron. Astrophys. Suppl., 129, 87.

Boyle B. J., Jones L. R., Shanks T., Marano B., Zitelli V., Zamorani G., 1991. In: ASP Conf. Ser. 21, The Space Distribution of Quasars, ed. Crampton D.

Boyle B. J., Wilkes B. J., Elvis M., 1997. Mon. Not. R. Astr. Soc., 285, 511.

Brandt W. N., Ward M. J., Fabian A. C., Hodge P. W., 1997. Mon. Not. R. Astr. Soc., 291, 709 .

Bregman J. N., Cox C. V., Tomisaka K., 1993. Ap. J. Lett., 415, L79

Briel U. G., et al., 1997. ROSAT User's Handbook, Max-Planck-Institut für Extraterrestrische Physik, Germany.

Buta R. J., McCall M. L., 1983. Mon. Not. R. Astr. Soc., 205, 131. 
Cappellaro E., 1996. In: IAU Sym. 171, New light on galaxy evolution, eds Bender R., Davies R. L.

Cardelli J. A., Clayton G. C., Mathis J. S., 1989a. Ap. J., 345, 245.

Cardelli J. A., Clayton G. C., Mathis J. S., 1989b. Ap. J., 345, 245.

Carone T. E., et al., 1996. Ap. J., 471, 737.

Chevalier R. A., Clegg A. W., 1985. Nature, 317, 44.

Chokshi A., Turner E. L., 1992. Mon. Not. R. Astr. Soc., 259, 421.

Cid Fernandes R., Plewa T., Rozyczka M., Franco J., Terlevich R., Tenorio-Tagle G., Miller W., 1996. Mon. Not. R. Astr. Soc., 283, 419.

Ciliegi P., Elvis M., Wilkes B. J., Boyle B. J., McMahon R. G., 1997. Mon. Not. R. Astr. Soc., 284, 401.

Cioffi D., 1990. In: Physical Processes in Hot Cosmic Plasmas., eds Brinkmann W., Fabian A., Giovannelli F., Phillips K.

Clavel J., et al., 1991. Ap. J., 366, 64.

Condon J. J., 1992. Ann. Rev. Astron. Astrophys., 30, 575.

Cordova F. A., 1995. In: X-ray binaries - Cambridge Astrophysics Series, Cambridge, MA: Cambridge University Press, eds Lewin W. H., Van Paradijs J., Van Den Heuvel E. P.

Cowie L. L., McKee C. F., Ostriker J. P., 1981. Ap. J., 247, 908.

Cowley A. P., Schmidtke P. C., Taylor V. A., MCGrath T. K., 1998. In: IAU Sym. 192, The Stellar Content of Local Group Galaxies, eds Whitelock P., Cannon R.

Crane P. C., Dickel J. R., Cowan J. J., 1992. Ap. J. Lett., 390, L9.

David L. P., Harnden F. R., Kearns K. E., Zombeck M. V., 1998. The ROSAT High Resolution Imager (HRI) Calibration Report, U.S. ROSAT Science Data Center/SAO.

Dietrich M., et al., 1994. Astron. Astrophys., 284, 33.

Done C., Ward M. J., Fabian A. C., Kunieda H., Tsuruta S., Lawrence A., Smith M. G., Wamsteker W., 1990. Mon. Not. R. Astr. Soc., 243, 713.

Dopita M. A., Koratkar A. P., Evans I. N., Allen M. G., Bicknell G. V., Sutherland R. S., Hawley J. F., Elaine S., 1996. In: ASP Conf. Ser. 103, The physics of LINERs in view of recent observations, eds Eracleous M., Koratkar A. P., Leitherer C., Ho L.

Dopita M. A., Koratkar A. P., Allen M. G., Tsvetanov Z. I., Ford H. C., Bicknell G. V., Sutherland R. S., 1997. Ap. J., 490, 202.

Drissen L., Roy J. R., 1996. In: ASP Conf. Ser. 98: From Stars to Galaxies: the Impact of Stellar Physics on Galaxy Evolution, eds Leitherer C., Fritze-Von Alvensleben U., 
Huchra J.

Edelson R. A., et al., 1996. Ap. J., 470, 364.

Edelson R. A., Malkan M. A., 1986. Ap. J., 308, 59.

Edelson R. A., Pike G. F., Krolik J. H., 1990. Ap. J., 359, 86.

Ehle M., Pietsch W., Beck R., Klein U., 1998. Astron. Astrophys., 329, 39.

Elson R. A. W., Fall S. M., Freeman K. C., 1989. Ap. J., 336, 734.

Elvis M., Wilkes B. J., McDowell J. C., Green R. F., Bechtold J., Willner S. P., Oey M. S., Polomski E., Cutri R., 1994. Ap. J. Suppl., 95, 1.

Engelbracht C. W., Rieke M. J., Rieke G. H., Kelly D. M., Achtermann J. M., 1998. Ap. J., 505, 639 .

Esin A. A., McClintock J. E., Narayan R., 1997. Ap. J., 489, 865.

Fabbiano G., Juda J. Z., 1997. Ap. J., 476, 666.

Fabbiano G., Panagia N., 1983. Ap. J., 266, 568.

Fabbiano G., Trinchieri G., 1984. Ap. J., 286, 491.

Fabbiano G., Trinchieri G., 1987. Ap. J., 315, 46.

Fabbiano G., Feigelson E., Zamorani G., 1982. Ap. J., 256, 397.

Fabbiano G., Kim D.-W., Trinchieri G., 1992. Ap. J. Suppl., 80, 531.

Fabbiano G., 1988a. Ap. J., 325, 544.

Fabbiano G., 1988b. Ap. J., 330, 672.

Fabbiano G., 1995. In: X-ray binaries, eds Lewin W. H., Van Paradijs J., Van Den Heuvel E. P.

Ferland G. J., Netzer H., 1983. Ap. J., 264, 105.

Filipovic M. D., 1998. In: IAU Sym. 192, The Stellar Content of Local Group Galaxies, eds Whitelock P., Cannon R.

Filippenko A. V., Sargent W. L. W., 1988. Ap. J., 324, 134.

Filippenko A. V., Sargent W. L. W., 1989. Ap. J. Lett., 342, L11.

Filippenko A. V., Terlevich R., 1992. Ap. J. Lett., 397, L79.

Filippenko A. V., Ho L. C., Sargent W. L. W., 1993. Ap. J. Lett., 410, L75.

Filippenko A. V., 1985. Ap. J., 289, 475.

Filippenko A. V., 1989. Astron. J., 97, 726. 
Filippenko A. V., 1992. In: ASP Conf. Ser. 31, Relationships Between Active Galactic Nuclei and Starburst Galaxies, ed. Filippenko A. V.

Ford H. C., Tsvetanov Z. I., Ferrarese L., Jaffe W., 1998. In: IAU Sym. 184, The Central Regions of the Galaxy and Galaxies, ed. Sofue Y.

Gallais P., Rouan D., Lacombe F., Tiphene D., Vauglin I., 1991. Astron. Astrophys., 243, 309.

Garcia-Vargas M., Bressan A., Diaz A., 1995. Astron. Astrophys. Suppl., 112, 13.

Gehrels N., 1986. Ap. J., 303, 336.

Gehren T., Fried J., Wehinger P. A., Wyckoff S., 1984. Ap. J., 278, 11.

Genzel R., Eckart A., Ott T., Eisenhauer F., 1997. Mon. Not. R. Astr. Soc., 291, 219.

Ghez A. M., et al., 1998. In: IAU Sym. 184, The Central Regions of the Galaxy and Galaxies, ed. Sofue Y.

Gonzalez-Delgado R. M., et al., 1994. Ap. J., 437, 239.

Haehnelt M. G., Rees M. J., 1993. Mon. Not. R. Astr. Soc., 263, 168.

Halpern J. P., Steiner J. E., 1983. Ap. J. Lett., 269, L37.

Hasinger G., Turner T. J., George I. M., Boese G., 1992. GSFC OGIP Calibration Memo CAL/ROS/92-00.

Hawkins M. R. S., 1996. Mon. Not. R. Astr. Soc., 278, 787.

Heckman T. M., Armus L., Miley G. K., 1990. Ap. J. Suppl., 74, 833.

Heckman T. M., Dahlem M., Eales S. A., Fabbiano G., Weaver K., 1996. Ap. J., 457, 616.

Heckman T. M., 1980. Astron. Astrophys., 87, 152.

Helfand D. J., 1984. Publ. Astron. Soc. Pacific, 96, 913.

Helfer T. T., Blitz L., 1997. Ap. J., 478, 162.

Ho L. C., Filippenko A. V., Sargent W. L. W., 1993. Ap. J., 417, 63.

Ho L. C., Filippenko A. V., Sargent W. L. W., 1995a. Ap. J., 98, 477.

Ho L. C., Filippenko A. V., Sargent W. L. W., 1995b. Ap. J. Suppl., 98, 477.

Ho L. C., Filippenko A. V., Sargent W. L. W., 1996. Ap. J., 462, 183.

Ho L. C., Filippenko A. V., Sargent W. L. W., 1997a. Ap. J. Suppl., 112, 315.

Ho L. C., Filippenko A. V., Sargent W. L. W., 1997b. Ap. J. Suppl., 112, 315.

Ho L. C., Filippenko A. V., Sargent W. L. W., 1997c. Ap. J. Suppl., 112, 391. 
Ho L. C., Filippenko A. V., Sargent W. L. W., 1997d. Ap. J., 487, 568.

Ho L., 1996. In: ASP Conf. Ser. 103, The physics of LINERs in view of recent observations, eds Eracleous M., Koratkar A. P., Leitherer C., Ho L.

Ho L., 1998. In: Observational Evidence for the Black Holes in the Universe, ed. Chakrabarti S. K.

Ho L. C., 1999. Ap. J., 516, 672.

Horne K., 1986. Publ. Astron. Soc. Pacific, 98, 609.

Huchra J., Burg R., 1992. Ap. J., 393, 90.

Hughes J. P., 1987. Ap. J., 314, 103.

Hunter D. A., Gillett F. C., Gallagher, J. S. I., Rice W. L., Low F. J., 1986. Ap. J., 303, 171.

Hurt R. L., Turner J. L., 1991. Ap. J., 377, 434.

Hurt R. L., Merrill K. M., Gatley I., Turner J. L., 1993. Astron. J., 105, 121.

Hurt R. L., 1994. Publ. Astron. Soc. Pacific, 106, 549.

Hutchings J. B., Crampton D., Campbell B., 1984. Ap. J., 280, 41.

Ishisaki Y., et al., 1996. Pub. Astr. Soc. Japan, 48, 237.

Johnson R., Lawrence A., Terlevich R., D. C., 1997. Mon. Not. R. Astr. Soc., 287, 333.

Johnson R. A., 1997. PhD thesis, Queen Mary and Westfiled College.

Junkes N., Zinnecker H., Hensler G., Dahlem M., Pietsch W., 1995. Astron. Astrophys., 294,8 .

Kaspi S., et al., 1996. Ap. J., 470, 336.

Kaspi S., Smith P. S., Maoz D., Netzer H., Jannuzi B. T., 1996. Ap. J. Lett., 471, L75.

Kassebaum T. M., Peterson B. M., Wanders I., Pogge R. W., Bertram R., Wagner R. M., 1997. Ap. J., 475, 106.

Keel W. C., 1983. Ap. J., 269, 466.

Kennicutt, R. C. J., 1983. Ap. J., 272, 54.

Kennicutt, Robert C. J., 1992. Ap. J., 388, 310.

Khachikian E. Y., Weedman D. W., 1974. Ap. J., 192, 581.

Kim D. W., Fabbiano G., Trinchieri G., 1992. Ap. J., 393, 134.

King I. T., 1985. Technical Report, La Palma, . 
Kinney A. L., Antonucci R. R. J., Ward M. J., Wilson A. S., Whittle M., 1991. Ap. J., $377,100$.

Koratkar A. P., Gaskell C. M., 1991. Ap. J. Lett., 370, L61.

Koratkar A., Deustua S. E., Heckman T., Filippenko A. V., Ho L. C., Rao M., 1995. Ap. J., 440,132 .

Kormendy J., Richstone D., 1995. Ann. Rev. Astron. Astrophys., 33, 581.

Kotilainen J. K., Ward M. J., 1994. Mon. Not. R. Astr. Soc., 266, 953.

Kraan-Korteweg R. C., Tammann G. A., 1979. Astron. Nachr., 300, 181.

Kraan-Korteweg R. C., 1986. Astron. Astrophys. Suppl., 66, 255.

Kriss G. A., Canizares C. R., Ricker G. R., 1980. Ap. J., 242, 492.

Kriss G. A., Hartig G. F., Armus L., Blair W. P., Caganoff S., Dressel L., 1991. Ap. J. Lett., 377, L13.

Kriss G. A., 1988. Ap. J., 324, 809.

Kunth D., Sargent W. L. W., Bothun G. D., 1987. Astron. J., 93, 29.

Kwan J., Krolik J. H., 1981. Ap. J., 250, 478.

Laor A., Fiore F., Elvis M., Wilkes B. J., McDowell J. C., 1994. Ap. J., 435, 611.

Laor A., Fiore F., Elvis M., Wilkes B. J., McDowell J. C., 1997. Ap. J., 477, 93.

Lauer T. R., et al., 1992. Astron. J., 104, 552.

Laver T. R., et al., 1993. Astron. J., 106, 1436.

Lawrence A., Rigopoulou D., Rowan-Robinson M., McMahon R. G., Broadhurst T., Lonsdale C. J., 1994. Mon. Not. R. Astr. Soc., 266, L41.

Lawrence A., Elvis M., Wilkes B. J., McHardy I., Brandt N., 1997. Mon. Not. R. Astr. Soc., 285, 879.

Lawrence A., 1987. Publ. Astron. Soc. Pacific, 99, 309.

Leitherer C., Heckman T. M., 1995. Ap. J. Suppl., 96, 9.

Leitherer C., 1996. In: ASP Conf. Ser. 98: From Stars to Galaxies: the Impact of Stellar Physics on Galaxy Evolution, eds Leitherer C., Fritze-Von Alvensleben U., Huchra J.

Linsky J. L., 1990. In: Imaging X-Ray Astronomy. A Decade of Einstein Observatory Achievements, ed. Elvis M.

Lira P., Lawrence A., O’Brien P., Johnson R. A., Terlevich R., Bannister N., 1999. Mon. Not. R. Astr. Soc, 305, 109. 
Long K. S., Gordon S. M., Blair W. P., Charle P. A., 1994. In: AIP Conference Proceedings: The soft X-ray cosmos, eds Schlegel E. M., Petre R.

Long K. S., Helfand D. J., Grabelsky D. A., 1981. Ap. J., 248, 925.

Lozinskaya T. A., 1992. Supernovae and stellar wind in the interstellar medium, New York: American Institute of Physics.

Lynds R., Tolstoy E., O’Neil., Earl J. J., Hunter D. A., 1998. Astron. J., 116, 146.

Mac Low M.-M., McCray R., 1988. Ap. J., 324, 776.

Mackie G., Fabbiano G., Kim D.-W., Ikebe Y., 1995. In: IAU Sym. 164, Stellar Populations, eds Van der Kruit P. C., Gilmore G.

Magorrian J., et al., 1998. Astron. J., 115, 2285.

Makishima K., et al., 1989. Pub. Astr. Soc. Japan, , 697.

Malkan M. A., 1983. Ap. J., 268, 582.

Maoz D., Koratkar A., Shields J. C., Ho L. C., Filippenko A. V., Sternberg A., 1998. Astron. J., 116, 55.

Maoz D., 1994. In: ASP Conf. Ser. 69, Reverberation Mapping of the Broad-Line Region in Active Galactic Nuclei, eds Gondhalekar K., Horne K., Peterson B. M.

Markert T. H., Donahue M. E., 1985. Ap. J., 297, 564.

Massey P., Strobel K., Barnes J. V., Anderson E., 1988. Ap. J., 328, 315.

Matonick D. M., Fesen R. A., 1997. Ap. J. Suppl., 112, 49.

Matonick D. M., Fesen R. A., Blair W. P., Long K. S., 1997. Ap. J. Suppl., 113, 333.

Matthews L. D., Gallagher, J. S. I., Krist J., Burrows C., Idt T. W., 1996. American Astronomical Society Meeting, 188, 403.

McLeod K. K., Rieke G. H., Rieke M. J., Kelly D. M., 1993. Ap. J., 412, 111.

McLeod K. K., 1997. In: Quasar Hosts, eds Clements D. L., Pérez-Fournon I.

McLure R. J., Dunlop J. S. K. M. J., Baum S. A., O'Dea C. P., Hughes D. H. 1998. Preprint, astro-ph/9809030.

Meurs E. J. A., Wilson A. S., 1984. Astron. Astrophys., 136, 206.

Miyoshi M., Moran J., Herrnstein J., Greenhill L., Nakai N., Diamond P., Inoue M., 1995. Nature, 373,127 .

Moorwood A. F. M., Van der Werf P. P., Kotilainen J. K., Marconi A., Oliva E., 1996. Astron. Astrophys., 308, L1.

Moran E. C., Halpern J. P., Helfand D. J., 1996. Ap. J. Suppl., 106, 341. 
Morrison R., McCammon D., 1983. Ap. J., 270, 119.

Morse J. A., 1994. Publ. Astron. Soc. Pacific, 106, 675.

Mushotzky R. F., Wandel A., 1989. Ap. J., 339, 674.

Mushotzky R. F., 1993. In: The Nearest Active Galaxies, eds Beckman J. E., Colina L., Netzer $\mathrm{H}$.

Nandra K., George I. M., Mushotzky R. F., Turner T. J., Yaqoob T., 1997. Ap. J., 476, 70.

Narayan R., Yi I., 1995. Ap. J., 452, 710.

Narayan R., Mahadevan R., Grindlay J. E., Popham R. G., Gammie C., 1998. Ap. J., 492,554 .

Narayan R., Mahadevan R., Quataert E., 1999. In: The Theory of Black Hole Accretion Discs, eds Abramowicz M. A., Bjornsson G., Pringle J. E.

Netzer H., Peterson B. M., 1997. In: Astronomical Time Series, eds Maoz D., Sternberg A., Leibowitz E. M.

Netzer H., Wamsteker W., Wills B. J., Wills D., 1985. Ap. J., 292, 143.

Nieto J.-L., Auriere M., 1982. Astron. Astrophys., 108, 334.

Okada K., Dotani T., Makishima K., Mitsuda K., Mihara T., 1998. Pub. Astr. Soc. Japan, $50,25$.

Oke J. B., 1990. Astron. J., 99, 1621.

Osterbrock D. E., Koski A. T., 1976. Mon. Not. R. Astr. Soc., 176, P61.

Osterbrock D. E., 1989. Astrophysics of gaseous nebulae and active galactic nuclei, Mill Valley, CA, University Science Books.

Papadakis I. E., Lawrence A., 1995. Mon. Not. R. Astr. Soc., 272, 161.

Papaderos P., Fricke K. J., Thuan T. X., Loose H.-H., 1994. Astron. Astrophys. Lett., 291, L13.

Peimbert M., Torres-Peimbert S., 1981. Ap. J., 245, 845.

Perley R. A., 1997. Very Large Array Observational Status Summary, U.S. National Radio Astronomy Observatory.

Peterson B. M., et al., 1991. Ap. J., 368, 119.

Peterson B. M., et al., 1994. Ap. J., 425, 622.

Peterson B. M., 1994. In: ASP Conf. Ser. 69, Reverberation Mapping of the Broad-Line Region in Active Galactic Nuclei, eds Gondhalekar K., Horne K., Peterson B. M. 
Petre R., Mushotzky R. F., Serlemitsos P. J., Jahoda K., Marshall F. E., 1993. Ap. J., $418,644+$.

Phillips M. M., Charles P. A., Baldwin J. A., 1983. Ap. J., 266, 485.

Plewa T., 1995. Mon. Not. R. Astr. Soc., 275, 143.

Press W. H., Teukolsky S. A., Vetterling W. T., Flannery B. P., 1992. Numerical Recipes, Cambridge University Press.

Primini F. A., Forman W., Jones C., 1993. Ap. J., 410, 615.

Ptak A., Serlemitsos P., Yaqoob T., Mushotzky R., Tsuru T., 1997. Astron. J., 113, 1286.

Ptak A., Yaqoob T., Mushotzky R., Serlemitsos P., Griffiths R., 1998. Ap. J. Lett., 501, L37.

Puchnarewicz E. M., Mason K. O., Romero-Colmenero E., Carrera F. J., Hasinger G., McMahon R., Mittaz J. P. D., Page M. J., Carballo R., 1996. Mon. Not. R. Astr. Soc., 281, 1243 .

Reichert G. A., et al., 1994. Ap. J., 425, 582.

Richstone D., et al., 1998. Nature, 395, A14.

Roberts T. P., Warwick R. S., Ohashi T., 1999. Mon. Not. R. Astr. Soc., 304, 52.

Robinson A., Binette L., Fosbury R. A. E., Tadhunter C. N., 1987. Mon. Not. R. Astr. Soc., 227, 97 .

Roeser S., Bastian U., 1991. PPM star catalogue : positions and proper motions of 181731 stars north of -2.5 degrees declination for equinox and epoch : J2000.0, Astronomisches Rechen-Institut by Spektrum Akademischer Verlag.

ROSAT Call for Proposals, 1991. Technical Appendix (Appendix F), Max-Planck-Institut Für Extraterrestrische Physik.

Rose J. A., Searle L., 1982. Ap. J., 253, 556.

Rosenblatt E. I., Malkan M. A., Sargent W. L. W., Readhead A. C. S., 1992. Ap. J. Suppl., 81, 59 .

Rowan-Robinson M., 1995. Mon. Not. R. Astr. Soc., 272, 737.

Roy J.-R., Aube M., McCall M. L., Dufour R. J., 1992. Ap. J., 386, 498.

Rubin V. C., Ford, K. W. J., 1986. Ap. J. Lett., 305, L35.

Sadler E. M., Jenkins C. R., Kotanyi C. G., 1989. Mon. Not. R. Astr. Soc., 240, 591.

Salpeter E. E., 1955. Ap. J., 121, 161.

Sandage A., Tammann G. A., 1981. A Revised Shapley-Ames Catalog of Bright Galaxies (RSA), Carnegi Institiution of Washington. 
Sarazin C. L., Bahcall J. N., 1977. Ap. J. Suppl., 34, 451.

Sauvage M., Vigroux L., 1991. In: IAU Sym. 148, The magellanic Clouds, eds Hyanes R., Milne D.

Schlegel D. J., Finkbeiner D. P., Davis M., 1998. Ap. J., 500, 525.

Schlegel E. M., 1994a. Astron. J., 108, 1893.

Schlegel E. M., 1994b. Ap. J. Lett., 424, L99.

Schlegel E. M., 1994c. Ap. J., 434, 523.

Schmitt J. H. M. M., 1990. Advances in Space Research, 10, 115.

Schulman E., Bregman J. N., 1995. Ap. J., 441, 568.

Serlemitsos P., Ptak A., Yaqoob T., 1996. In: ASP Conf. Ser. 103, The physics of LINERs in view of recent observations, eds Eracleous M., Koratkar A. P., Leitherer C., Ho L.

Shields J. C., Filippenko A. V., 1992. In: ASP Conf. Ser. 31: Relationships Between Active Galactic Nuclei and Starburst Galaxies, 267, ed. Filippenko A. V.

Shuder J. M., 1981. Ap. J., 244, 12.

Snowden S. L., Pietsch W., 1995. Ap. J., 452, 627.

Soltan A., 1982. Mon. Not. R. Astr. Soc., 200, 115.

Sramek R., 1992. In: ASP Conf. Ser. 31: Relationships Between Active Galactic Nuclei and Starburst Galaxies, 273, ed. Filippenko A. V.

Srinivasan G., 1997. In: Saas-Fee Advanced Course 25: Stellar remnants, eds Kawaler S. D., Novikov I., Srinivasan G.

Stark A. A., Gammie C. F., Wilson R. W., Bally J., Linke R. A., Heiles C., Hurwitz M., 1992. Ap. J. Suppl., 79, 77.

Stauffer J. R., 1982. Ap. J., 262, 66.

Stickland D. J., Lloyd C., Pike C. D., Walker E. N., 1987. The Observatory, 107, 74.

Takano M., Mitsuda K., Fukazawa Y., Nagase F., 1994. Ap. J. Lett., 436, L47.

Tanaka Y., et al., 1995. Nature, 375, 659.

Taniguchi Y., Ohyama Y., Yamada T., Mouri H., Yoshida M., 1996. Ap. J., 467, 215.

Telesco C. M., 1988. Ann. Rev. Astron. Astrophys., 26, 343.

Terlevich R., Melnick J., 1985. Mon. Not. R. Astr. Soc., 213, 841.

Terlevich E., Diaz A. I., Terlevich R., 1990. Mon. Not. R. Astr. Soc., 242, 271. 
Terlevich R., Tenorio-Tagle G., Franco J., Melnick J., 1992. Mon. Not. R. Astr. Soc., 255, 713.

Terlevich R., Tenorio-Tagle G., Rozyczka M., Franco J., Melnick J., 1995. Mon. Not. R. Astr. Soc., 272, 198.

Terlevich R., 1994. In: Circumstellar Media in the Late Stages of Stellar Evolution, eds Clegg R. E. S., Stevens I. R., Meikle W. P. S.

Tomisaka K., Bregman J. N., 1993. Pub. Astr. Soc. Japan, 45, 513.

Trinchieri G., Fabbiano G., 1991. Ap. J., 382, 82.

Trinchieri G., Fabbiano G., Paulumbo G. G. C., 1985. Ap. J., 290, 96.

Trinchieri G., Fabbiano G., Romaine S., 1990. Ap. J., 356, 110.

Tully R. B., Boesgaard A. M., Dyck H. M., Schempp W. V., 1981. Ap. J., 246, 38.

Turner J. L., Ho P. T. P., 1994. Ap. J., 421, 122.

Van Der Marel R. P., De Zeeuw P. T., Rix H.-W., 1997. Ap. J., 488, 119.

Van der Marel R. P., 1998. In: IAU Sym 186, Galaxy Interactions at Low and High Redshift, eds Sanders D. B., Barnes J.

Van Dyk S. D., Hamuy M., Filippenko A. V., 1996. Astron. J., 111, 2017.

Van Paradijs J., McClintock J., 1995. In: X-ray binaries - Cambridge Astrophysics Series, Cambridge, MA: Cambridge University Press, eds Lewin W. H., Van Paradijs J., Van Den Heuvel E. P.

Van Paradijs J., White N., 1995. Ap. J. Lett., 447, L33.

Van Paradijs J., 1995. In: X-ray binaries - Cambridge Astrophysics Series, Cambridge, MA: Cambridge University Press, eds Lewin W. H., Van Paradijs J., Van Den Heuvel E. P.

Vanbeveren D., De Loore C., Van Rensbergen W., 1998. Astron. Astrophys. Review, 9, 63.

Veilleux S., Osterbrock D. E., 1987. Ap. J. Suppl., 63, 295.

Vogler A., Pietsch W., 1996. Astron. Astrophys., 311, 35.

Vogler A., Pietsch W., 1999. Astron. Astrophys., 342, 101.

Walter R., Fink H. H., 1993. Astron. Astrophys., 274, 105.

Wandel A., 1991. Astron. Astrophys., 241, 5.

Wanders I., Peterson B. M., 1996. Ap. J., 466, 174.

Wanders I., Peterson B. M., Pogge R. W., Derobertis M. M., Van Groningen E., 1992. 
Astron. Astrophys., 266, 72 .

Watson M. G., Stanger V., Griffiths R. E., 1984. Ap. J., 286, 144.

Watson M. G., 1990. In: Windows on galaxies, eds Fabbiano G., Gallagher J. S., Renzini A.

White N. E., Nagase F., Parmar A. N., 1995. In: X-ray binaries - Cambridge Astrophysics Series, Cambridge, MA: Cambridge University Press, eds Lewin W. H., Van Paradijs J., Van Den Heuvel E. P.

Whittet D. C. B., Bode M. F., Murdin P., 1987. Vistas in Astronomy, 30, 135.

Williams R. M., Chu Y.-H., 1995. Ap. J., 439, 132.

Wills B. J., Netzer H., Wills D., 1985. Ap. J., 288, 94.

Wilson A. S., Ward M. J., Haniff C. A., 1988. Ap. J., 334, 121.

Wray J. D., 1988. The Color Atlas of Galaxies, Cambridge University Press.

Yee H. K., 1980. Ap. J., 241, 894.

Yee H. K., 1992. In: ASP Conf. Ser. 31, Relationships Between Active Galactic Nuclei and Starburst Galaxies, ed. Filippenko A. V.

Zang Z., Warwick R. S., Meurs E. J. A., 1997. Irish Astronomical Journal, 24, 45. 